\title{
Physicians Oral Abstracts
}

EBMT 2012

\section{Van Bekkum Award}

54

TCR gene editing results in effective immunotherapy of leukaemia without the development of GvHD

E. Provasi (1), P. Genovese (2), A. Lombardo (2), Z. Magnani (1), L. Pei-qi (3), A. Reik (3), V. Chu (3), D. Paschon (3), L. Zhang (3), J. Kuball (4), B. Camisa (1), A. Bondanza (1), G. Casorati (1), M. Ponzoni (1), F. Ciceri (1), C. Bordignon (5), P. Greenberg (4), M. Holmes (3), P. Gregory (3), L. Naldini (2), C. Bonini (1)

(1)San Raffaele Scientific Institute (Milan, IT); (2)San Raffaele Telethon Institute for Gene Therapy (Milan, IT); (3)Sangamo Biosciences (Richmond, US); (4)Fred Hutchinson Cancer Research Center and University of Washington (Seattle, US); (5)San Raffaele University (Milan, IT)

T cell receptor (TCR) gene-transfer in T lymphocytes is a promising tool for adoptive immunotherapy of cancer patients for whom natural tumor-specific lymphocytes cannot be isolated. Nevertheless, TCR-transferred $T$ lymphocytes differ from their natural counterpart in carrying two different TCRs and this limits their efficacy and alters their toxicity profile due to reduced expression of tumor-specific TCR and inappropriate pairing of TCR chains. To overcome these issues, we developed a novel approach aimed at the complete genetic editing of $\mathrm{T}$ cell specificity, based on the ZFN-mediated disruption of the endogenous TCR chain genes coupled to the transfer of genes encoding for a tumor-specific TCR. We selected Wilms' tumor antigen 1 (WT1) as a model antigen. For the complete editing of T cell specificity, we established a protocol to sequentially disrupt the endogenous TCR alpha and beta chain genes with high efficiency (averages: $34 \% \pm 11$ and $16 \% \pm 10$ ), and to permanently transfer WT1-specific TCR alpha and beta chain genes by lentiviral vectors (average efficiencies: $53 \% \pm 25$ and $21 \% \pm 15$ ). This procedure resulted in a population of TCRedited lymphocytes encoding only the tumor-specific TCR that, in the absence of competition from the endogenous receptor, was expressed at high levels. Accordingly, TCR-edited lymphocytes were superior to conventional TCR-transferred cells in promoting specific recognition of WT1-expressing targets, including primary leukemias, and most importantly, were devoid of residual endogenous TCR reactivity including alloreactivity. Finally, for a comprehensive assessment of safety and antitumor efficacy, we treated immunodeficient mice, infused with primary human leukemias, with matched TCR-transferred, TCRedited, and unmanipulated cells and we monitored mice for Graft-versus-host disease (GvHD) and leukemia appearance. Mice treated with TCR-edited cells showed higher event free survival than TCR-transferred $(p<0.05)$ or unmanipulated lymphocytes $(p<0.01)$. These data demonstrate that the successful genetic re-programming of $\mathrm{T}$ cell specificity in primary lymphocytes results in a functionally superior target specific killing activity and thus has the potential to greatly improve the safety and therapeutic activity of cancer immunotherapy. (Provasi and Genovese: equal contribution).

\section{Presidential Symposium}

\section{6}

Basic Science Award

Nerve growth factor and collagen 1 prevent apoptosis and maintain durable self-renewal of adult mouse haematopoietic stem cells stimulated with proliferative cytokines

S. Woehrer (1), D. Knapp (2), K. Rowe (2), H. Mader (2), M. Copley (2), C. Benz (2), D. Kent (2), R. Oostendorp (3), C. Eaves (2)

(1)Medical University of Vienna (Vienna, AT); (2)Terry Fox Laboratory (Vancouver, CA); (3)Klinikum rechts der Isar (Munich, DE)

Objectives: Self-renewal is a key property of hematopoietic stem cells (HSCs) and the hallmark of stem cells in general. However, despite decades of research, it is still unknown which extrinsic factors are necessary to optimize HSCs self-renewal ex vivo. The present experiments were designed to identify potential stromal-derived factors that promote mouse HSC selfrenewal ex vivo.

Methods: Highly purified HSCs isolated from mouse adult bone marrow (i.e. EPCR+, CD150+, CD48-, CD45+ cells, 42\% having longterm repopulating ability) were cultured for 7 days in vitro in serum-free medium (SFM) and various combinations of cytokines, stromal cells and factors they produce. HSCs numbers were determined by performing limiting dilution transplants in sub-lethally irradiated congenic W41/W41 mice assessed for repopulation activity of the transplanted cells 4-6 months post-transplant. Culture conditions that supported the greatest expansion of HSCs were used to assess the HSC output of single input cells and to design comparative gene expression analyses on 6-hour-stimulated cells to identify potential stromal factors involved. These were then tested for their potential to replicate the effects of stroma cells and stromal cell conditioned medium.

Results: Of the various conditions initially tested, the addition of stromal cells or stromal cell-conditioned medium (CM) to Steel factor and IL-11 gave maximal and equivalent HSC outputs after 7 days. (5- to 11-fold expansion of transplantable HSC numbers). This indicated a stromal cell contact-independent mechanism. Visual tracking of single-cell cultures revealed that stromal cell-derived factors prevented the apoptosis of $\sim 50 \%$ of the input cells and transplant assays of the CM-containg cultures showed that $90 \%$ of the cells that produced at least one daughter HSC did so asymmetrically and only $10 \%$ did so symmetrically. Gene expression analysis showed that pathways activated by collagen 1 (Col1) and nerve growth factor (NGF) were significantly upregulated during the self-renewing process and a direct test of these two factors combined indicated they could replace the activity of $\mathrm{CM}$ in promoting HSC survival and expansion

Conclusion: NGF and Col1 are key stromal-derived factors that can positively regulate adult HSC expansion when these are stimulated with proliferative cytokines. 
077

Outcome of matched sibling donor SCT is equivalent to unrelated well matched donor HSCT. A report from the prospective international multicentre trial

ALL-SCT-BFM 2003

C. Peters, A. Schrauder, A. Stackelberg, M. Schrappe, P. Bader, W. Ebell, R. Handgretinger, K. Sykora, J. Schrum, B. Kremens, K. Ehlert, M. Albert, R. Meisel, T. Güngör, W. Holter, B. Strahm, B. Gruhn, A. Schulz, U. Pötschger, M. Zimmermann, T. Klingebiel on behalf of BFM and PDWP

The BFM-Study group initiated a prospective international multicentre trial for allogeneic haematopoietic stem cell transplantation (HSCT) in children and adolescents with ALL. The trial aims were to answer whether HSCT from a matched sibling donor (MSD) was equivalent to HSCT from a $9 / 10$ or 10/10 HLA matched donor (MD) and to determine the efficacy and efficiency of HSCT from HLA-mismatched donors (MMD).

Between September 2003 and September 2009, 387 patients were transplanted in 27 participating HSCT centres. Mean age of the patients at HSCT was 10 years (range 0.5 to 18). Indications for HSCT were poor response to induction treatment, cytogenetic aberrations $[t(9 ; 22), t(4 ; 11)]$, early bone marrow relapse, or any subsequent ALL relapse. 97 patients received a MSD-HSCT, 251 patients a MD-HSCT, and 39 patients a MMDHSCT. The backbone conditioning regimen was TBI/etoposide. GvHD prophylaxis consisted of CSA for MSD, and CSA plus MTX and ATG-F for MD, T/B-cell depletion was performed for MMD-HSCT. Results: Median follow-up was 2.4 years. Acute GvHD Grade III and IV occurred in $10 \%$ of all patients. The 2-year cumulative incidence of extensive chronic GvHD was $15 \%$ after MSD and $12 \%$ after MD-HSCT. The 4 -year probability of event-free survival ( $p E F S$ ) after MSD-HSCT was equivalent to MD-HSCT ( $70 \%$ vs. $68 \%$; $p=0.37,95 \%$ confidence interval for the difference ranges from $-8 \%$ to $+10 \%$ ).

The cumulative incidence of treatment related mortality (TRM) after 1 -year was $5 \%$ for MSD and $8 \%$ for MD-HSCT (n.s.). The 2 -year cumulative incidence of relapse was 18\% after MSDHSCT and $20 \%$ after MD-HSCT (n.s.). For patients with very high relapse risk the results for MD/MSD-HSCT $(n=187)$ and MMD HSCT $(n=39)$ differed significantly (2-year pEFS $68 \%$ vs. $28 \%$; $p<0.001)$; the 1 -year incidence of TRM was $8 \%$ after MD/ MSD-HSCT and $22 \%$ after MMD-HSCT $(p=0.04)$. In multivariate analysis for event free survival, no statistically significant difference was seen for patients older than 12 years, T-ALL, $\mathrm{BCR}-\mathrm{ABL}+$ and MLL-rearrangement. We demonstrate that allogeneic HSCT from well-matched unrelated donors or genoidentical sibling donors is an effective treatment with acceptable toxicity. Precise HLA typing and matching and use of ATG-F resulted in a low incidence of extensive chronic GvHD, which is an important achievement for the quality of life in children and adolescents. The results from this trial demonstrate the feasibility of a harmonized HSCT approach across multiple international centres.
078

A phase III PETHEMA/GEM randomised trial of posttransplant maintenance in multiple myeloma: superiority of bortezomib

L. Rosiñol (1), M.T. Cibeira (1), M.V. Mateos (2), J. Martínez (3), A. Oriol (4), A.I. Teruel (5), D. Hernández (6), J. López Jiménez (7), J. de la Rubia (8), M. Granell (9), J. Besalduch (10), L. Palomera (11), Y. González (12), M.A. Etxebeste (13), J. DíazMediavilla (14), M. Hernández (15), F. de Arriba (16), A. Alegre (17), J.J. Lahuerta (3), J. San Miguel (2), J. Bladé (1) (1)Hospital Clinic (Barcelona, ES); (2)Hospital Clinico (Salamanca, ES); (3)Hospital 12 de Octubre (Madrid, ES); (4)Hospital Germans Trias i Pujol (Badalona, ES); (5)Hospital Clinico (Valencia, ES); (6)Hospital La Paz (Madrid, ES); (7)Hospital Ramón y Cajal (Madrid, ES); (8)Hospital $\mathrm{La} \mathrm{Fe}$ (Valencia, ES); (9)Hospital Sant Pau (Barcelona, ES); (10)Hospital Son Espases (Palma de Mallorca, ES); (11)Hospital Lozano Blesa (Zaragoza, ES); (12)Hospital Josep Trueta (Girona, ES); (13)Hospital de Donostia (Donostia, ES); (14)Hospital Clinico San Carlos (Madrid, ES); (15)Hospital Universitario de Canarias (La Laguna, ES); (16)Hospital Morales Messeguer (Murcia, ES); (17)Hospital La Prinesa (Madrid, ES)

Introduction: In April 2006, the Spanish Myeloma Group (PETHEMA/GEM) activated a randomized phase III trial comparing induction with TD vs. VTD vs. VBMCP/VBAD/Bortezomib in patients 65 years-old or younger with newly diagnosed symptomatic MM and ASCT with MEL-200 followed by maintenance with thalidomide/bortezomib (TV) vs. thalidomide (T) vs. alfa-2b-interferon (IFN).

Primary end points: The primary end-point was time to progression from the initiation of maintenance therapy.

Patients and Methods: The maintenance program consisted of TV (thalidomide $100 \mathrm{mg}$ daily plus one cycle of bortezomib-1.3 $\mathrm{mg} / \mathrm{m}^{2}$ on days $1,4,8$ and 11 every 3 months) versus T (single agent thalidomide at a dose of $100 \mathrm{mg}$ daily) versus IFN (subcutaneous IFN at a dose of $3 \mathrm{MU}$ three times per week). The planned maintenance duration was three years or until disease progression or toxicity. From February 1, 2007 to January 27, 2011266 patients were randomized to maintenance therapy (TV:90; T: 89, IFN: 87). Response and survival were evaluated on an intention-to-treat basis. Responses and progressions reported by the investigators were centrally reassessed.

Results: The patient's characteristics at diagnosis such as age, ISS stage, cytogenetics and presence of extramedullary plasmacytomas as well as induction regimen and diagnosis-randomization interval were similarly distributed among the 3 arms. The response status at the time of randomization after ASCT was CR: $51 \%$, VGPR: $23 \%$, PR: $24 \%$ and SD: $2 \%$ and was well balanced in the three groups. The CR rate with maintenance was improved by $23 \%$ with TV, $11 \%$ with T and $19 \%$ with IFN $(p=N S)$. After a median follow-up of 24 months, the PFS was significantly longer with TV compared with T and IFN (PFS at 2 yrs: $78 \%$ vs. $63 \%$ vs. $49 \%$, p=0.01). OS was not significantly different among the 3 arms. Grade 3 and 4 hematological toxicity was similar $(22.2 \%$ vs. $16 \%$ vs. $21.8 \%)$. No peripheral neuropathy (PN) was observed with IFN being its frequency similar with TV $(12.2 \%)$ and T $(10.1 \%)$. No grade IV PN was observed. Dose reductions for TV, T and IFN were required in $33.3 \%, 33.7 \%$ and $19.5 \%$ of the patients, respectively. The discontinuation rate due to toxicity was significantly higher with thalidomide compared with TV $(30.3 \%$ vs. $15.6 \%, p=0.08)$ and with IFN $(30.3 \%$ vs. $18.3 \%, p=0.17)$.

Conclusion: The addition of bortezomib to thalidomide maintenance resulted in a significantly longer PFS when compared with thalidomide alone or with IFN with no increased toxicity. 
079

Allo-HLA-DQ/-DP specific CD4+ T-cells for adoptive immunotherapy of leukaemia

A. Bloetz (1), E. Distler (1), E. Schnürer (1), F. Schier (2), M. Theobald (1), W. Herr (1)

(1)3rd Dept. of Medicine (Mainz, DE); (2)Dept. of Pediatric Surgery (Mainz, DE)

In allogeneic hematopoietic stem cell transplantation (alloHSCT) patient and donor are usually matched for the HLA class I molecules A/B/C as well as for the HLA class II molecules DRB1 and DQB1. In contrast, the HLA-DPB1 locus is still ignored in donor selection. Clinical studies have demonstrated that disparities at HLA-DQB1 and distinct HLA-DPB1 alleles do not adversely affect the outcome of allo-HSCT. It has also been shown that HLA class II is predominantly expressed on hematopoietic cells under non-inflammatory conditions. Thus CD4+ donor T cells recognizing patient-derived HLA-DQB1 or permissive HLA-DPB1 mismatch alleles may primarily target leukemic and hematopoietic cells, while sparing non-hematopoietic tissues. We used PBMC of healthy donors to generate mature monocyte-derived dendritic cells (DC), which underwent transfection with in vitro transcribed RNA coding for single HLA-DQ/-DP mismatch alleles by electroporation. These alloHLA expressing DC were used to stimulate autologous naive CD4+ CD45RA+ T cells in mixed lymphocyte reactions (MLR) in vitro. Rapidly expanding MLR cells showed specific recognition of allo-HLA-DQ/-DP molecules as demonstrated by lack of immune reactivity to non-transfected DC as well as by complete inhibition of alloreactivity using HLA allele-specific antibodies. The allo-HLA-DQ/-DP specific T cells were also analyzed for reactivity to a broad panel of primary acute myeloid leukemia (AML) blasts. Strong IFN-g secretion could be observed only for those AML targets that carried the HLA allele used for T cell priming, demonstrating the specificity of this approach. We further investigated HLA class II expression on hematopoietic and non-hematopoietic cells by flow cytometry. HLA class II was not detected on primary fibroblasts, keratinocytes, and normal kidney cells (each $n=10$ ), but was expressed at significant levels on primary AML blasts and B-cell lines (each $n=10$ ). Expression levels followed the hierarchy: $D P>D R>D Q$. Up-regulation of HLA class II expression was observed on all cell types after pre-incubation with IFN-g, but not after addition of TNF-a, IL-1b and IL-6. Our approach appears suitable for generating alloHLA-DQ/-DP specific CD4+ T cell lines that recognize leukemia cells while presumably sparing non-hematopoietic cells under non-inflammatory conditions. It may be of potential use in adoptive immunotherapy of allo-HSCT patients who express single HLA-DQ or permissive HLA-DP mismatch alleles.

\section{0}

Improved survival in AML patients with a high FLT3-ITD mutation rate after allogeneic transplantation G. Ehninger (1), M. Bornhäuser (1), M. Kramer (1), C. Röllig (1), H. Wandt (2), M. Haenel (3), H. Einsele (4), W. Aulitzky (5), N. Schmidt (6), W. Berdel (7), M. Stelljes (7), C. Baldus (8), A. Mackensen (9), U. Platzbecker (1), M. Schaich (1), C. Thiede (1), A. Ho (10), J. Schetelig (1)

(1)University (Dresden, DE); (2)Internal Medicine (Nuremberg, $D E) ; ~(3) K l i n i k u m$ (Chemnitz, DE); (4)University (Würzburg, DE); (5)RBK Hospital (Stuttgart, DE); (6)St. Georg Hospital (Hamburg, DE); (7)University (Münster, DE); (8)University (Berlin, DE); (9)University (Erlangen, DE); (10)University (Heidelberg, DE)

The ratio of the FLT3-ITD mutation to the wt-FLT3 allele has significant prognostic importance of FLT3-ITD mutations in AML (Thiede C et al., Blood 2002). There is still uncertainty about the role of allogeneic transplantation (allo-SCT) in the treatment of patients with FLT-ITD mutation. In order to proof the presence of an allogeneic effect we compared the survival after allo-SCT in first remission in patients with a mutant rate $<0.8$ with those $\geq 0.8$. For comparison, the results in patients cohorts treated with chemotherapy alone with the same mutations rates were analyzed.

Patients and Methods: Patients diagnosed with AML, aged 18-60 years, and treated in the AML 2003 trial of the SAL were analyzed. According to the risk-adapted treatment strategy of the trial, cytogenetically intermediate-risk (IR) and adverserisk (AR) patients should receive an allo SCT as consolidation treatment if a HLA-matched-sibling donor (IR) or HLA-matched related or unrelated donor (AR) was available. Patients with no available donor received high-dose cytarabine based consolidation or autologous SCT. Survival analyses were performed by using the Kaplan-Meier method including log-rank tests for significance testing.

Results: Of 1182 patients enrolled in the trial, 257 were FLT3ITD+ (22\%). The ratio of the FLT3-ITD mutation to the wt-FLT3 allele was $<0.8$ in 182 patients and $\geq 0.8$ in 75 patients. 47 $(26 \%)$ of the low mutation rate group and $30(40 \%)$ of the high mutation rate group received an allogeneic transplantation. In the cohorts having received an allogeneic transplantation, the 3-year disease-free survival (DFS) in the mutant rate $<0.8$ and $\geq 0.8$ groups was $58 \%$ and $50 \%$, respectively $(p=0.53)$. The 3 -year overall survival (OS) was $61 \%$ and $59 \%$, respectively $(p=0.47)$. In the cohorts having chemotherapy as consolidation, the 3-year DFS in the mutant rate $<0.8$ and $\geq 0.8$ groups was $36 \%$ and $10 \%$, respectively $(p<0.001)$. The 3 -year OS was $43 \%$ and $11 \%$, respectively $(p<0.001)$.

Overall, allo-SCT lead to an overall and event-free survival in patients with a high FLT3-ITD mutation rate comparable with those with a low mutation rate. Without allogeneic transplantation and chemotherapy alone, again significant differences in DFS and OS between patients with a high vs. low mutation rate as in our report of the previous AML96 could be shown. These data point toward a strong allogeneic effect after transplantation thus eliminating the negatve impact of a high mutation rate.

\section{1}

The outcome of patients with follicular lymphoma in the rituximab era treated with autologous stem cell transplant according to the high-dose regimen received. A retrospective study of the EBMT Lymphoma Working Party

I. El-Najiar, A. Boumendil, J. Luan, C. Thieblemont, D. Blaise, K. Thomson, M. Mohty, P. Colombat, A. Rambaldi, J.P. Jouet, P. Biron, M. Martelli, H. Tilly, D. Pohlreich, M. Pfreundschuh, C. Cordonnier, C. Crawley, J.Y. Cahn, J.P. Vernant, J. Gribben, G. Cook, N. Russell, A. Ferrant, P. Dreger, S. Montoto on behalf of the EBMT lymphoma working party

Aim: The aim of this study was to investigate the outcome of patients with FL having ASCT according to the high-dose regimen (TBI vs. BEAM) and previous treatment with rituximab.

Patients and Methods: Between 1995 and 2007, 7910 patients with FL had their 1st ASCT and were reported to the EBMT registry. A full data set was available for 2233 patients who had ASCT with either TBI containing regimens or BEAM, which constitute the study group. The following outcome measures were assessed by univariate and multivariate comparisons: overall survival (OS), event free survival (EFS), non-relapse mortality (NRM) and incidence of relapse (IR) from the time of ASCT.

Results: A total of 680 patients received a TBI-containing regimen, and 1553 patients BEAM. Before ASCT 713 patients $(32 \%)$ had been treated with monoclonal antibodies (MoAb). Patients who had TBI were younger (median age: 47 ) than patients who underwent BEAM (median age: 49; $p<0.001$ ), and were transplanted in 1st remission more frequently $(61 \%)$ than BEAM patients $(44 \%, p<0.001)$. In contrast, more patients treated with BEAM had received MoAb prior to ASCT (37\%) than TBI patients $(21 \%, p<0.001)$. Peripheral blood was the source of stem cells in $98 \%$ of patients having BEAM in comparison with $92 \%$ of patients having TBI $(p<0.001)$. After a median follow-up of 60 months, the median overall survival (OS) for the 
whole group was 146 months and the median EFS, 72 months. The multivariate analysis revealed the following significant risk factors, as shown in attached table. In addition there was a trend for patients receiving TBI to have a better OS than patients receiving BEAM $(p=0.06)$.

Conclusions: In contrast to observations made in patients autografted before 1995 (Montoto Leukemia 21:2324, 2007), in this series form a more recent time period (but with a shorter followup) the beneficial effect of TBI over BEAM in terms of relapse prevention was not counteracted by an increased NRM, resulting in a significantly better EFS with TBI in the rituximab era The use of MoAb (rituximab) prior to ASCT had no detrimental impact on outcome and in fact was associated with significantly better OS and EFS.

\section{Working Party Session Akute Leukaemia}

\section{5}

Introduction and update on the EBMT Acute Leukaemia Working Party activities

M. Mohty, M. Labopin, S. Giebel on behalf of the ALWP of EBMT

Transplant activity for acute leukemia continues to increase worldwide. In the ALWP registry 82500 transplant procedures for AML and ALL (auto and allo-HSCT) were registered thus far.

[081]

\begin{tabular}{|c|c|c|}
\hline Risk factors & $\begin{array}{l}\text { Relativ e risk } \\
\text { (95\% CI) }\end{array}$ & P-ralue \\
\hline \multicolumn{3}{|l|}{ OS } \\
\hline TBI versus BEAM & $0.8(0.7-1.0)$ & 0.06 \\
\hline $\begin{array}{l}\text { Disease status at BMT (all categ ories vs CR1PR1,VGPR1) } \\
\text { Primary refractory, stable disease, relapse, progression } \\
C R>1, P R>1 \\
\text { Unlonown }\end{array}$ & $\begin{array}{l}1.4(1.1-1.8) \\
0.9(0.7-1.2) \\
1.2(0.8-1.8)\end{array}$ & $\begin{array}{l}0.001 \\
0.6 \\
0.5\end{array}$ \\
\hline Age (for 1 year increase) & $1.02(1.01-1.03)$ & $<0.001$ \\
\hline Previcus MoAb vs none & $0.7(0.6-0.9)$ & 0.003 \\
\hline Time from diagnos is to ASCT >1 year (vs < lyear) & $1.3(1.02-1.6)$ & 0.03 \\
\hline$\overline{B M}$ vs $\mathrm{PB}$ & $1.7(1.2-2.3)$ & $<0.001$ \\
\hline \multicolumn{3}{|l|}{ EFS } \\
\hline TBI v BEAM & $0.7(0.6-0.8)$ & $<0.001$ \\
\hline Age (for 1 year increase) & $1.0(1.0-1.01)$ & 0.04 \\
\hline Previcus MoAb vs none & $0.8(0.7-0.9)$ & 0.009 \\
\hline BMvs PB & $1.3(1.0-1.7)$ & 0.04 \\
\hline \multicolumn{3}{|l|}{ NRM } \\
\hline Age (for 1 year increase) & $1.0(1.0-1.04)$ & 0.04 \\
\hline Time from diagnos is to ASCT > l yea (vs < lyear) & $1.6(1.04-2.5)$ & 0.03 \\
\hline Female vs male & $0.7(0.5-0.9)$ & 0.02 \\
\hline Previcus $\mathrm{MoAb}$ vs none & $0.6(0.4-0.8)$ & 0.005 \\
\hline BMvs PB & $2.0(1.2-3.6)$ & 0.007 \\
\hline \multicolumn{3}{|l|}{ IR } \\
\hline TBI v BEAM & $0.7(0.6-0.8)$ & $\angle 0.001$ \\
\hline $\begin{array}{l}\text { Disease status at BMT (all categ cries vs CR1PRI,VGPR1) } \\
\text { Primary refractory, s table disease, relapse, progression } \\
C R>1, P R>1 \\
\text { Unlonown }\end{array}$ & $\begin{array}{l}1.5(1.3-1.9) \\
1.0(0.8-1.3) \\
1.0(0.7-1.5)\end{array}$ & $\begin{array}{l}<0.001 \\
0.8 \\
0.8\end{array}$ \\
\hline
\end{tabular}


The ALWP objectives are:

(i) to organize high level accredited educational activities pertinent to acute leukemia (latest symposiums: Nantes in 2008, Barcelona in 2009, Milan in 2010, and Warsaw in 2011):

(ii) to design and support prospective clinical trials in the field of acute leukemia across member centres (the pan-european elderly AML randomized trial is currently recruiting patients: ClinicalTrials.gov Identifier: NCT00766779);

(iii) to generate high quality retrospective studies addressing different issues related to acute leukemia management and therapy;

(iv) to increase within the EBMT registry the quality of data pertinent to HSCT for acute leukemia; and

(v) to generate guidelines pertinent to the management of acute leukemia.

Currently, the ALWP activities are organized and structured within 6 subcommittees (SC) focused on specific fields of interest: autologous HSCT SC, Immunotherapy SC, Alternative donors SC, RIC SC, Molecular markers SC, and the Developing centers SC.

The ALWP is currently chaired by M. Mohty (France) and the secretary is S. Gieble (Poland) and includes representatives/ members from most EBMT centres/countries, with expertise in both auto and allogeneic HSCT for AML and ALL. The ALWP meets twice a year to discuss ongoing studies and new study proposals and review manuscript preparation. All EBMT members are encouraged to submit study proposals (registry-based studies) to the ALWP. After a quick feasibility assessment performed by the ALWP office, projects will be discussed during the ALWP winter (usually October or November) and spring (during the EBMT annual meeting) business meetings.

\section{6}

Intravenous Busulfan versus TBI conditioning for allo-HSCT: where do we stand?

A. Nagler, M. Labopin, E. Polge, M. Mohty on behalf of the RIC Subcommittee of the ALWP of EBMT

Allogeneic hematopoietic stem cell transplantation (allo-HSCT) is the treatment of choice for high and intermediate risk acute myeloid leukemia (AML). The traditional preparative myeloablative regimens include Cyclophosphamide (Cy) combined with high-dose total body irradiation (TBI) or Cy combined with Busulfan (Bu) (Bu/Cy regimen).

Registry studies as well as randomized studies compared the two types of preparative regimens for allo-HSCT in patients with AML with some conflicting results concerning outcome and toxicity. The original goal of the Bu/Cy protocol was to reduce toxicity, improve outcome and provide an alternative for patients that received prior radiation and would thus not be a suitable candidates for TBI. However, the risk of veno-occlusive disease of the liver (VOD) was still a matter of concern. Indeed, VOD was shown to be relatively high in patients that were conditioned with high dose of oral Busulfan. In contrast, IV Bu has a more favorable toxicity profile as compared to the oral formulation. It is thus conceivable that the risk of hepatic VOD as well as transplant-related mortality (TRM) would be reduced when using the IV formulation of Bu, leading to improved allo-HSCT outcome.

In order to address this issue, the ALWP of the EBMT recently performed a series of different retrospective registry-based studies comparing IV Bu/Cy to Cy/TBI for conditioning prior to alloHSCT for adult patients with AML in complete remission, first relapse and resistant relapse. In all, the IV Bu/Cy conditioning regimen resulted in similar transplantation outcomes including engraftment, non-relapse mortality (NRM), relapse incidence (RI), overall survival (OS) and leukemia-free survival (LFS) in comparison to the TBI/CY preparative regimen. Acute GVHD was significantly lower in AML patients in CR who received IV $\mathrm{Bu} / \mathrm{Cy} v s$. TBI/Cy conditioning, respectively. Moreover, in AML patients in first relapse undergoing allo-HSCT, IV Bu/Cy conditioning can result in higher post-transplant remission rates which resulted in better LFS and OS in comparison to TBI/Cy conditioning. This advantage in favor of the IV Bu/Cy regimen is likely due to lower overall toxicity and improved capacity for salvage therapy.

In summary, the available research evidence strongly suggests a favorable safety and efficacy profile in favor of the IV Bu/Cy regimen. Thus, the future role of TBI in allo-HSCT for AML needs to be revisited in well designed controlled studies.

\section{7}

Molecular markers and allo-HSCT for AML: the latest developments

J. Esteve, M. Labopin, S. Brunet, A. Pigneux, M. BrandsNijenhuis, H. Schouten, E. Polge, N.-C. Gorin, M. Mohty on behalf of the molecular markers Subcommittee of the ALWP of EBMT

Allo-HSCT in early phase is considered the treatment of choice for younger patients with high risk AML, although this presumed benefit of allo-HSCT has not been analyzed in molecularly defined AML subtypes. In this context, several studies within MMS-ALWP have been conducted to elucidate the role of allo-HSCT for specific AML entities. Main results of these studies, performed in patients allografted in CR1, will the summarized:

- AML with FLT3-ITD: the effect of FLT3-ITD was analyzed in a cohort of 206 patients with normal cytogenetics AML. FLT3ITD $(n=120)$ was associated to a higher relapse incidence (RI, 5-yr RI: $30 \%$ vs. 16\%) and decreased LFS (5-yr LFS: $58 \%$ vs. $71 \%$ ).

- AML with MLL rearrangement (MLL-r, $n=138)$ : type of MLL rearrangement had a strong prognostic impact, allowing the distinction between favorable MLL-r entities, such as $t(9 ; 11)$ and $\mathrm{t}(11 ; 19)$, and unfavorable MLL-r subtypes, namely $\mathrm{t}(6 ; 11)$ and $t(10 ; 11)$. Patients from the favorable subgroup showed a significantly better OS (2-yr OS: $70 \%$ vs. $29 \%$ ) due to a markedly reduced $\mathrm{RI}(20-25 \%$ vs. $46-47 \%)$.

- AML with monosomal karyotype (MK-AML, $n=189$ ): MK-AML showed an independent impact after alloHSCT, with a 3-yr LFS and RI of $24 \%$ and $55 \%$, respectively. Of note, outcome of patients with MRC-defined poor cytogenetics after excluding MKAML and $7 q$ abnormalities did not differ from that of patients with intermediate-risk cytogenetic AML (IR-AML).

- AML with t(6;9) AML: 73 patients allografted in CR1 were analyzed, with a 5 -yr OS of $55 \pm 6 \%$, similar to IR-AML patients.

In summary, the outcome following allo-HSCT of patients with high risk AML varies significantly depending on the specific entity considered. Identification of molecularly-defined AML subtypes with a high relapse risk after allo-HSCT justifies the design of strategies for a preemptive intervention after transplantation.

\section{8}

Autologous HSCT for acute lymphoblastic leukaemia: is it a valid option?

N.-C. Gorin, M. Labopin, S. Giebel, M. Mohty on behalf of the autologous HSCT Subcommittee of the ALWP

In the early days of autologous bone marrow transplantation (ASCT) for acute leukemias, leukemia free survivals over $50 \%$ at 3 years have been reported for adult ALL both with marrow purged with cyclophosphamide derivatives and with unpurged marrow. A very interesting finding was that engraftment of neutrophils and platelets was rapid in sharp contrast with acute myelocytic leukemias where the kinetics of hematopoietic recovery were considerably delayed.

Most teams have long term survivors (more than 10 years follow up), some autografted despite poor risk prognostic factors such as $\mathrm{t}(4 ; 11)$ or $\mathrm{t}(9 ; 22)$. Moreover, small series have advocated in favor of the introduction of maintenance chemotherapy following ASCT. However, several prospective studies have failed 
to demonstrate a clear advantage of ASCT over conventionaldose chemotherapy and the relapse rate following ASCT has been considered to be too high for further prospective studies. More recent analyses demonstrated that results of ASCT may depend strongly on the level of minimal residual disease (MRD) and in the era of routine MRD monitoring the interest in ASCT is re-growing. Here, we will update the current status of ASCT in adult ALL using the EBMT ALWP data base and make proposals for new studies with MRD monitoring.

\section{9}

"Non-transplant" treatment approaches for AML

F. Ravandi (Houston, US)

Despite considerable progress in the treatment of acute myeloid leukemia in the past several decades, the prognosis of the majority of patients with this disease remains guarded. Advances in supportive care and better characterization of disease subsets through cytogenetics and molecular analysis have led to significant success in treating specific subsets of patients such as those with acute promyelocytic leukemia (APL) and core binding factor leukemias (CBF), particularly among the younger patients who are able to better tolerate the effects of cytotoxic chemotherapy. However, overall, only about $40 \%$ of younger patients and less than $10 \%$ of older patients with this disease are alive at 5 years. Current research is focusing on the identification of new cellular targets amenable to specific inhibitors, designing the best strategies for combining these novel agents with traditional chemotherapy regimens, and determining prognostic indicators that may allow us to better stratify therapy.

\section{0 \\ "To RIC or not to RIC" for AML: that is the question! J. Cornelissen (Rotterdam, NL)}

Allogeneic HSCT is generally advocated as the treatment of choice to consolidate remission in intermediate and poor risk AML. The last decade, new cytogenetic and molecular markers have been identified that specifically relate to poor or very poor-risk AML. The latter categories include the so-called monosomal karyotype (MK) leukemia's and those with high expression of EVI-1. Recent studies suggested that the beneficial effect of allo-HSCT also applies to those categories of AML. Despite being more efficacious than consolidation chemotherapy, the risk of relapse in poor-risk AML is still considerable and may estimate between 30 and $50 \%$. It suggests that, although clearly operational, the GVL effect has not been exploited fully. Further improvement may be pursued by avoiding delay and proceeding to allo-HSCT before full peripheral hematopoietic recovery. In addition, the continuous application of new agents such as 5-azacytidine after allo-HSCT, may offer a new approach to facilitate ongoing GVL. Reduced intensity conditioning regimen have been developed in order to reduce NRM. Several studies have indeed shown a lower NRM after RIC conditioning as compared to MA conditioning, especially as patients assigned to RIC regimens were most times older and had higher comorbidity scores. While several retrospective studies have suggested a somewhat higher relapse rate in recipients of RIC allo-HSCT, some very recent studies suggested that relapse may not differ in intensively pretreated AML CR1 patients, who subsequently proceeded to either RIC allo-HSCT or MA allo-HSCT. Collectively, these studies may suggest that the beneficial effect of allo-HSCT largely depends on the immunotherapeutic GVL effect and less on the intensity of the conditioning regimen in AML patients, who attained first CR upon intensive chemotherapy.

\section{Working Party Session Late Effects}

97

Quality of life of patients undergoing an allogeneic SCT: An interim Analysis of the prospective LEWP study of the EBMT comparing RIC and conventional intensity conditioning

J. Weis, A. Görög, G. Socié, H. Bertz, J. Passweg, M.T. van Lint on behalf of the LEWP of the EBMT

Background: In the last years allogeneic stem cell transplants after reduced intensity conditioning (RIC) have become increasingly popular. As a result of minimized cytotoxic conditioning and therefore lower early treatment related mortality and morbidity, allogeneic transplantation can now be considered in patients who would otherwise not qualify for this treatment. As the success of the transplantation relies mainly on the graft-versustumour effect, which often goes in parallel with a more or less distinct graft-versus-host reaction, long term complications and problems in various aspects of quality of life (QoL) have to be expected as they are already known from conventional stem cell transplantation HSCT.

Aims of the study: The aim of the study is to compare quality of life in both groups (reduced vs. conventional conditioning) in the longitudinal course over three years after transplantation. We want to analyse QoL after non-myeloablative HSCT over the long term outcome.

Methods: Based on a prospective longitudinal design we assess QoL as primary endpoint with a validated questionnaire (EORTC QoL-C30, EORTC HDC26). Secondary end points are transplant related mortality, overall survival, event free survival and graft versus host disease. We are measuring QoL before transplantation (T0), 100 days after (T1) and each year over a prospected time frame of 3 year (T2 1 year, T3 2 years and T4 3 years). It is planned to reach a final sample size of 300 patients in each group at T0. The study is carried out as a multicenter study recruiting patients in four centres (France, Germany, Italy and Switzerland).

Results: As the study is still ongoing, the focus of this presentation is an interim analysis of the data collected until end of 2011. We present results on QoL and medical data from 218 patients (37.2\% standard; $62.8 \%$ reduced conditioning), showing that also patients with RIC have substantial problems in various functions and activities of daily living over time.

Discussion: Due to high drop out rates further centres will be included to improve patient recruitment and guarantee substantial subgroup samples over the longitudinal course of the study.

\section{8}

Early recognition of female genital graft-versus-host disease to avoid severe complications: a study of 32 cases

M. Leclerc, P. Hirsch, M. Rybojad, A. Petropoulou, M. Robin, P. Ribaud, R. Peffault de la Tour, B. Cavelier-Balloy, D. VexiauRobert, G. Socié

Hôpital Saint Louis (Paris, FR)

Objectives: Genital chronic graft-versus-host disease (cGVHD) is a complication of allogeneic hematopoietic stem-cell transplantation (alloHSCT) responsible for significant morbidity and impairment of quality-of-life, but remains under-diagnosed. We conducted a retrospective monocentric study to describe the main characteristics of women diagnosed with genital cGVHD and to evaluate the impact of a systematic gynecological follow-up after alloHSCT on genital cGVHD diagnosis, treatment and evolution. Methods: Women seen at the gynecology consultation of our alloHSCT center, between 2008 and 2010 were included if they had received alloHSCT after year 2000 and were diagnosed with genital lesions of cGVHD. The diagnosis could be clinical or histological in atypical cases. All patients received local 
hormonal therapy for 2 months before introduction of local steroids as first-line treatment of genital cGVHD, in order to exclude concomitant estrogen deficiency.

Results: We identified 32 women with genital cGVHD. Data regarding alloHSCTs are listed in Table 1. Six women were allografted before 2008. Gynecological follow-up after alloHSCT was systematic in our institution since year 2008. In the 2008-2010 period, among 138 women who received alloHSCT, 26 (19\%) developed genital cGVHD. Patients mostly complained about vaginal dryness, dyspareunia and vaginal narrowing. Genital cGVHD was classified as grade I in $16(50 \%)$ cases, grade II in $3(9 \%)$ cases and grade III in $13(41 \%)$ cases. We identified local mucosal paleness as a new kind of grade I lesion. Interestingly, median time before first gynecological consultation after alloHSCT was 232 days for patients with grade III lesions versus 111 days for patients with grade I lesions. In 23 (72\%) women, cGVHD involved other organs, mainly mouth (59\%), skin (44\%) and eyes (44\%). With treatment of genital cGVHD, 11 patients had stabilization of lesions, 14 had partial remission and 3 had complete remission (Table 2). Most of them could resume sexual activity. Among the 4 patients who progressed under treatment, 3 were initially diagnosed with grade III disease and later needed surgery.

Conclusion: Genital cGVHD is frequent among women who receive alloHSCT, especially in case of other cutaneous or mucous locations of cGVHD. A systematic gynecological followup after alloHSCT, allowing early diagnosis of genital cGVHD, may help avoid severe complications, as local therapy seems to be more effective in grade I lesions.

\section{9}

Male genital changes and their implications in sexual life after allogeneic haematopoietic stem cell transplantation S.M. Müller, P. Häusermann, A. Rovo, J. Halter, J. Passweg, A. Tichelli

University Hospital Basel (Basel, CH)

Background and Methods: in women, genital chronic graftversus-host disease ( $g c G v H D$ ) is well described, but only limited data with regard to male recipients after HSCT exist. In a prospective cross-sectional single center study, we aimed to address this issue in post HSCT male recipients performing a whole body skin examination, focusing on genital changes. Furthermore posttransplant sexual contentedness and sexual functioning were assessed by two self-assessment questionnaires: the 5-Item Version of the International Index of Erectile Function (IIEF-5) and the modified Brief Sexual Symptom Checklist (mBSSC).

\begin{tabular}{|c|c|}
\hline Age at transplantation (years): median (range) & $40(16-60)$ \\
\hline Parity : median (range) & $2(0.4)$ \\
\hline \multicolumn{2}{|l|}{ Hematological diagnosis: $n(\%)$} \\
\hline Hodgkin lymphoma & $3(9)$ \\
\hline Non Hodgkin Lymphoma & $3(9)$ \\
\hline Chronic Lymphoid Leukemia & $1(3)$ \\
\hline Multiple Myeloma & $2(6)$ \\
\hline Acute Lymphoid Leukemia & $2(6)$ \\
\hline Acute Myeioid Leukemia & $12(38)$ \\
\hline Chronic Myeloid Leukemia & $2(6)$ \\
\hline Myelofibrosis & $3(9)$ \\
\hline Severe Aplastic Anemia & $2(6)$ \\
\hline Other & $2(6)$ \\
\hline \multicolumn{2}{|l|}{ Donor: $\mathbf{n}(\%)$} \\
\hline Sibling & $18(56)$ \\
\hline Unrelated & $14(44)$ \\
\hline \multicolumn{2}{|l|}{ Graft type: $n(\%)$} \\
\hline Bone marrow & $9(28)$ \\
\hline Peripheral blood & $20(63)$ \\
\hline Cord blood & $3(9)$ \\
\hline \multicolumn{2}{|l|}{ Conditioning: $\mathbf{n}(\%)$} \\
\hline Myeloablative & $15(47)$ \\
\hline Non myeloablative & $17(53)$ \\
\hline \multicolumn{2}{|l|}{ CD34 infused $\left(\times 10^{6} / \mathrm{kg}\right)$ : median (range) } \\
\hline Bone marrow & $4.81(0.8-4.32)$ \\
\hline Peripheral blood & $7.72(5.2-20.69)$ \\
\hline Cord blood & $0.22(0.12-0.23)$ \\
\hline \multicolumn{2}{|l|}{ GVHD prophylaxis: $n(\%)$} \\
\hline $\operatorname{CS} A+M T X$ & $14(44)$ \\
\hline$C S A+M M F$ & $17(53)$ \\
\hline Cs $A+$ steroids & $1(3)$ \\
\hline
\end{tabular}

Table 1: Patients' characteristics at alloHSCT

\begin{tabular}{ccccc}
\hline Initial severity & $\begin{array}{c}\text { Stabilization } \\
\mathrm{n}(\%)\end{array}$ & $\begin{array}{c}\text { Partial Remission } \\
\mathrm{n}(\%)\end{array}$ & $\begin{array}{c}\text { Complete Remission } \\
\mathrm{n}(\%)\end{array}$ & $\begin{array}{c}\text { Progression } \\
\mathrm{n}(\%)\end{array}$ \\
\hline I $(\mathrm{n}=16)$ & $6(38)$ & $7(44)$ & $2(12)$ & $1(6)$ \\
II $(\mathrm{n}=3)$ & $1(33)$ & $2(67)$ & $0(0)$ & $0(0)$ \\
III $(\mathrm{n}=13)$ & $4(31)$ & $5(38)$ & $1(8)$ & $3(23)$ \\
\hline
\end{tabular}

Table 2: Clinical evolution of gynecological lesions with medical treatment 
Results: all 155 asked patients accepted to participate. The characteristics of the study population are listed in Table 1 . The median time between HSCT and genital examination was 50 months (range 21-72). Thirty-one out of 155 patients (19\%) showed remarkable genital skin changes, 21/155 patients $(13 \%)$ had gcGvHD-related inflammatory genital lesions (12 had Zoon's-like balanoposthitis; 6 lichen sclerosus-like lesions; 5 phimosis; 2 patients had more than one feature). Patients with gcGvHD had significantly higher coincidence of oral mucosal $(p<0.0001)$, ocular mucosal $(p<0.002)$ and non-mucosal cutaneous cGvHD $(p<0.026)$ compared to patients without gcGvHD (Table 2). Further posttransplant genital lesions included unspecific balanitis, hyperpigmentation and melanotic macules of the glans penis, vitiligo and penis deviation. The rate of questionnaire returning was $51 \%(79 / 155)$ for the mBSSC and $65 \%$ for the IIEF-5 (88/155); $64 \%$ out of the patients, who returned the $\mathrm{mBSSC}$ reported to be uncontented with sexual functioning. The major sexual problem reported was erectile dysfunction affecting $58 / 88(65 \%)$ of those who returned the IIEF-5. Only 9 out of 21 patients with gcGvHD returned the questionnaires, reporting no relevant uncontentedness or sexual dysfunction.
This small return rate does not allow further interpretation in this particular aspect.

Conclusions: the high participation in this study reflects the great interest of posttransplant male recipients regarding genital issues. gcGvHD was observed in $13 \%$ of the evaluated population mostly in correlation with mucosal and non-mucosal skin cGVHD. Erectile dysfunction was the most prevalent sexual problem reported. Transplantation centers should be aware of possible male genital changes and their implications in sexual life. A regular screening should be standard part of the post-transplant control.

100

Uptake and outcome of assisted reproductive techniqes in long-term survivors

N. Salooja, A. Babb, K. Lindsay, J. Apperley

Hammersmith Hospital (London, UK)

We have audited the uptake of artificial reproductive techniques amongst allogeneic transplant patient in a single

[99]

Table 1: Patients' characteristics

\begin{tabular}{|c|c|}
\hline \multicolumn{2}{|l|}{ General information } \\
\hline Total number of patients & 155 \\
\hline Median age at HSCT (range); years & $42(12-68)$ \\
\hline Median age at time of control (range); years & $50(21-72)$ \\
\hline Median time interval between HSCT and control (range); months & $71(11-364)$ \\
\hline $\begin{array}{l}\text { Disease } \\
\text { - Acute leukemia } \\
\text { - Chronic myeloid leukemia } \\
\text { - Myelodvsplastic syndrome, moveloproliferative neoplasms. } \\
\text { - Myeloma } \\
\text { - Chronic Iymphocytic leukemia, (Non-) Hodgkin's disease } \\
\text { - Others }\end{array}$ & $\begin{array}{l}64(41 \%) \\
23(15 \%) \\
17(11 \%) \\
9(6 \%) \\
22(14 \%) \\
20(28 \%)\end{array}$ \\
\hline $\begin{array}{l}\text { Disease status } \\
\text { - Standard } \\
\text { - High risk }\end{array}$ & $\begin{array}{l}106(73 \%) \\
40(27 \%)\end{array}$ \\
\hline Total body irradiation & $81(57 \%)$ \\
\hline $\begin{array}{l}\text { Acute GVHD } \\
\text { - Grade 0-I } \\
\text { - Grade II-IV }\end{array}$ & $\begin{array}{l}77(57 \%) \\
58(43 \%)\end{array}$ \\
\hline $\begin{array}{l}\text { Chropic GvHHD } \\
\text { - None } \\
\text { - Yes }\end{array}$ & $\begin{array}{c}40(28 \%) \\
103(72 \%)\end{array}$ \\
\hline \multicolumn{2}{|l|}{ Genital changes } \\
\hline $\begin{array}{l}\text { Absent } \\
\text { Present }\end{array}$ & $\begin{array}{l}124(81 \%) \\
31(19 \%)\end{array}$ \\
\hline $\begin{array}{l}\text { 1.) Genital chronic GXHD } \\
\text { Rosttransplant Zoon's balanoposthitis-like } \\
\text { Rostransplant phimoosis } \\
\text { Postransplant lichen sclerosis-like }\end{array}$ & $\begin{array}{l}21(14 \%) \\
12(8 \%) \\
4(3 \%) \\
5(3 \%)\end{array}$ \\
\hline $\begin{array}{l}\text { 2.) Other genital features } \\
\text { Melanotic macules on the glans. } \\
\text { Hvoernigrnentation of the glans } \\
\text { Unspecific initative balinitis } \\
\text { Rostinflammatox } \\
\text { Genital vitiligo } \\
\text { Deviation of the penis }\end{array}$ & $\begin{array}{c}10(6 \%) \\
3 \\
2 \\
2 \\
1 \\
1 \\
1\end{array}$ \\
\hline
\end{tabular}


Table 2: Comparison of patients without versus with genital chronic graft-versus-host disease

\begin{tabular}{|c|c|c|c|}
\hline Parameter & $\begin{array}{l}\text { Patients without } \\
\text { genital cG } \mathrm{yHQ}\end{array}$ & $\begin{array}{l}\text { Patients with } \\
\text { genital ç } \mathrm{x} \text { HD }\end{array}$ & $\mathrm{p}$-value \\
\hline Number of patients & 134 & 21 & - \\
\hline Total body irradiation & $71(53 \%)$ & $10(48 \%)$ & 0.599 \\
\hline \multicolumn{4}{|l|}{ Disease } \\
\hline - Acute leukemia & $56(42 \%)$ & $8(38 \%)$ & \\
\hline - Chronic myeloid leukemia & $20(15 \%)$ & $3(14 \%)$ & \\
\hline $\begin{array}{l}\text { - Myeladvsplastic syndrome, } \\
\text { myeloproliferative neoplasms }\end{array}$ & $14(10 \%)$ & $3(14 \%)$ & 0.958 \\
\hline - Myelorna & $7(5 \%)$ & $2(9 \%)$ & \\
\hline $\begin{array}{c}\text { - Chronic lymphocytic leukemia, } \\
\text { (non-Hodgkin's disease }\end{array}$ & $19(14 \%)$ & $3(14 \%)$ & \\
\hline - Others & $18(13 \%)$ & $2(9 \%)$ & \\
\hline $\begin{array}{l}\text { Disease status } \\
\text { - } \quad \text { Standard } \\
-\quad \text { High risk }\end{array}$ & $\begin{array}{l}92(73 \%) \\
34(27 \%)\end{array}$ & $\begin{array}{l}14(70 \%) \\
6(30 \%)\end{array}$ & 0.779 \\
\hline $\begin{array}{l}\text { Acute GVVHD } \\
-\quad \text { Grade 0-I } \\
-\quad \text { Grade II-IV }\end{array}$ & $\begin{array}{l}68(57 \%) \\
51(43 \%)\end{array}$ & $\begin{array}{c}9(56 \%) \\
7(44(\%)\end{array}$ & 0.946 \\
\hline $\begin{array}{l}\text { Charanic GyHR } \\
-\quad \text { None } \\
-\quad \text { present }\end{array}$ & $\begin{array}{l}39(31 \%) \\
85(\%)\end{array}$ & $\begin{array}{c}1(5 \%) \\
18(95 \%)\end{array}$ & 0.018 \\
\hline Oral mucosal chronic GovHR. & $23(17 \%)$ & $14(67 \%)$ & $<0.0001$ \\
\hline Ocular chronic SVWHR & $9(8 \%)$ & $5(36 \%)$ & 0.002 \\
\hline $\begin{array}{l}\text { Non-mucosal cutaneous chronic SVWHR } \\
\text { (diagnostic features) }\end{array}$ & $7(5)$ & $6(28 \%)$ & 0.026 \\
\hline
\end{tabular}

centre. In addition we have investigated the frequency of use and the outcome of attempting parenthood using stored material. A postal questionnaire was sent to 434 patients surviving a minimum of 2 years. 221 patients responded including 112 male patients and 72 female patients under the age of 42. Among male patients, 42 of 112 banked sperm and 25 subsequently attempted parenthood. Of 72 women within a reproductive age group, 12 stored either embroys and-or eggs and-or ovarian tissue. Following transplant 5 of 8 women with cryopreserved embryos used them to attempt pregnancy, one in a surrogate.

Not everyone was offered the opportunity to store tissue and the reasons for this will be discussed together with outcome data of using stored material.

\section{1}

Increase of suicidal and accidental deaths after haematopoietic stem cell transplantation: from the Late Effects Working Party of the EBMT

A. Tichelli, M. Labopin, A. Rovó, M. Badoglio, M. Arat, M.T. van Lint, A. Lawitschka, C.P. Schwarze, J. Passweg, G. Socié on behalf of the Late Effects Working Party of the EBMT

Background: Relapse and transplant related complications are leading causes of mortality after hematopoietic stem cell transplantation (HSCT). Suicides and accidents have not been studied in details.

Methods: This retrospective observational study was based on 294 '922 patients who underwent autologous or allogeneic HSCT from 1980 to 2009. The primary endpoints were the cumulative incidence of death from suicide and accident, the deaths rates by suicide and accident, as well as the standardized mortality ratio (SMR) and the absolute excess risk (AER) of these causes of death. To define factors associated with suicide or death from accident, a case control analysis was performed.

Findings: In total 116,149 (39.4\%) died. From these deaths, 189 were due to suicide and 125 to an accident. The 10 year cumulative incidence of death by suicide and by accident was 101.8 and 55.6 per 100,000 patients, respectively. There were 20.7 suicidal deaths (males 27.4; females; 14.0) and 13.7 accidental death (18.0 in males; 8.2 in females) per 100,000 personyears. Based on the Eurostat statistics,, the expected suicidal and accidental death rates per 100,000 person-years were 9.2 (males 14.0; females 4.4) and 10.5 (males 16.3; females 4.9), respectively. The SMR and the AER of suicide after HSCT were $2.12(p<0.001)$, and 10.91 higher than in the European general population for 100,000 deaths, respectively. The SMR and AER of accident were $1.23(p<0.05)$ and 2.54 , respectively (Table 1). In a case-control study, relapses were significantly more frequent among patients who commit suicide after autologous HSCT ( $37 \%$ vs. 18\%; $p<0.0001)$. Chronic graft-versushost disease (GVHD) was significantly higher among patients who committed suicides after allogeneic HSCT, as compared to controls $(64 \%$ vs. $37 \% ; p=0.001$ ) (Table 2 ).

Interpretation: This study demonstrates that there is an excess of deaths due to suicide and to a lesser extend to accidents after HSCT as compared to the European general population. Relapse was associated with more deaths by suicide and accident after autologous HSCT and chronic GVHD with more deaths by suicide after allogeneic HSCT. Patient at risk for suicide or accident after HSCT should be recognized and preventative strategies initiated. 

Mortality Ratio (SMR) and Absolute Excess Ratio (AER) per 100'000 for suicides and accidents, including al patients after HSCT and stratified by sex

\begin{tabular}{|c|c|c|c|c|c|c|}
\hline Population & Person-years & $\begin{array}{l}\text { Suicide rates } \\
\text { per } 100000\end{array}$ & $\begin{array}{l}\text { Expected } \\
\text { suicide rates }\end{array}$ & $O / E$ & SMR $(95 \% \mathrm{C})$ & $\begin{array}{c}\text { AER } \\
\left.\text { (for } 100^{\prime} 000\right)\end{array}$ \\
\hline \multicolumn{7}{|c|}{ Deaths by suicide } \\
\hline Overall & $915 ' 183$ & 20.7 & 9.2 & $189 / 89$ & $2.12(1.83-2.45)$ & 10.91 \\
\hline Male & 5107700 & 27.4 & 14.0 & $140 / 71$ & $1.96(1.65-2.31)$ & 13.41 \\
\hline Female & $401^{\prime} 401$ & 12.2 & 4.4 & $49 / 18$ & $2.77(2.05-3.67)$ & 7.81 \\
\hline \multicolumn{7}{|c|}{ Deaths by accident } \\
\hline Overall & $915^{\prime} 183$ & 13.7 & 10.5 & $125 / 102$ & $1.23(1.02-1.46)$ & 2.54 \\
\hline Male & $510^{\prime} 700$ & 18.0 & 16.3 & $92 / 83$ & $1.11(0.89-1.36)$ & 0.98 \\
\hline Female & $401 \% 401$ & 8.2 & 4.6 & $33 / 18$ & $1.83(1.26-2.57)$ & 1.64 \\
\hline
\end{tabular}

Table 2: Characteristics of cases and controls - nested case-control study for the risk of suicide and accidents. Case control matching criteria included type of HSCT (allogeneic versus autologous), age at HSCT by decade, sex of the patients, year of transplantation, and length of follow-up.

\begin{tabular}{|l|c|c|c|c|c|c|}
\hline & \multicolumn{5}{|c|}{ Suicide death } & \multicolumn{3}{c|}{ Accident death } \\
\hline & $\begin{array}{c}\text { Cases } \\
187\end{array}$ & $\begin{array}{c}\text { Controls } \\
560\end{array}$ & p-value & $\begin{array}{c}\text { Cases } \\
124\end{array}$ & $\begin{array}{c}\text { Controls } \\
372\end{array}$ & p-value \\
\hline \multicolumn{7}{|c|}{ All patients treated with HSCT } \\
\hline $\begin{array}{l}\text { High risk at } \\
\text { HSCT }\end{array}$ & $76(44 \%)$ & $233(45 \%)$ & 0.8 & $47(39 \%)$ & $138(39 \%)$ & 0.92 \\
\hline Relapse & $52(28 \%)$ & $81(15 \%)$ & $<0.0001$ & $26(21 \%)$ & $45(12 \%)$ & 0.02 \\
\hline \multicolumn{7}{|c|}{ Patients treated with allogeneic HSCT } \\
\hline High risk & & & $11(24 \%)$ & $23(17 \%)$ & 0.28 \\
\hline Family donor & $49(69 \%)$ & $143(67 \%)$ & 0.81 & $38(84 \%)$ & $111(80 \%)$ & 0.5 \\
\hline RIC & $12(18 \%)$ & $36(18 \%)$ & 0.96 & $8(17 \%)$ & $27(19 \%)$ & 0.73 \\
\hline relapse & $10(14 \%)$ & $21(10 \%)$ & 0.33 & $5(10 \%)$ & $12(9 \%)$ & 0.7 \\
\hline Acute GVHD & $28(41 \%)$ & $62(32 \%)$ & 0.2 & $17(38 \%)$ & $38(29 \%)$ & 0.26 \\
\hline Chronic GVHD & $29(64 \%)$ & $62(37 \%)$ & 0.001 & $14(44 \%)$ & $39(38 \%)$ & 0.53 \\
\hline TBI in MAC & $34(62 \%)$ & $106(65 \%)$ & 0.63 & $26(72 \%)$ & $65(61 \%)$ & 0.22 \\
\hline \multicolumn{7}{|c|}{ Patients treated with autologous HSCT } \\
\hline High risk & $65(59 \%)$ & $179(56 \%)$ & 0.57 & $36(49 \%)$ & $105(52 \%)$ & 0.59 \\
\hline Relapse & $42(37 \%)$ & $60(18 \%)$ & $<0.0001$ & $21(28 \%)$ & $33(14 \%)$ & 0.009 \\
\hline TBI & $17(16 \%)$ & $43(14 \%)$ & 0.5 & $6(8 \%)$ & $23(11 \%)$ & 0.58 \\
\hline
\end{tabular}

\section{Autoimmune Diseases}

112

Autoimmune disease stem cell transplantation: an appraisal

A. Tyndall on behalf of the EULAR and EBMT

There is now a 15 year experience with hematopoietic stem cell transplantation for treating severe autoimmune disease. In addition, a newly evolving field of mesenchymal stem cell therapy of autoimmune disease is evolving.

Phase I/II studies $(1,360$ patients in the EBMT/EULAR data base and 130 patients in the CIBMTR data base) using autologous HSCT in several major autoimmune disease have shown a satisfactory benefit risk ratio. Over one-third of patients achieved a durable relative drug free remission with a treatment-related mortality of around $8-10 \%$. ranging from $12 \%$ for SLE and $2 \%$ for multiple sclerosis). Large phase III randomized controlled trials are advanced in systemic sclerosis (ASTIS - 156 patients and SCOT - 75 patients Trials) and Crohn's disease (ASTIC Trial - 48 patients) as well as non- randomised phase II study in MS (HALT). Results should be become available in 2012, but so far no unexpected TRM has been observed. A small randomised study in SSc (ASSIST - 19 patients ) was positive.

In systemic sclerosis, data of the past 12 months suggest remodelling of collagen and normalization of microvasculature after hematopoietic stem cell transplantation, a new finding. In MS and SLE durable remission was observed in some patients despite full immune reconstitution. 
The interpretation of the existing trials and planning of future studies needs to take into account an evolving situation regarding improving transplantation techniques and alternate less toxic treatment options for autoimmune disease e.g. biologics. Mesenchymal stem cells have shown promise in exerting an immediate anti-inflammatory immunomodulatory role in some autoimmune disease with little evidence of acute toxicity. Large randomised prospective trials are needed to confirm the promising in vitro, in vivo animal model and small anecdotal clinical data.

\section{Acute Graft-versus-host Disease}

\section{8}

Polyclonal anti-thymocyte globulins for the prophylaxis of graft-versus-host disease after allogeneic haematopoietic stem cell transplantation - a systematic meta-analysis

S. Theurich, H. Fischmann, A. Shimabukuro-Vornhagen, J. Chemnitz, U. Holtick, C. Scheid, N. Skoetz, M. von BergweltBaildon

University Hospital Cologne (Cologne, DE)

Introduction: Graft-versus-host-disease (GVHD) is a complication frequently occurring after HSCT and a potentially life threatening condition. One strategy for the prevention of GVHD is the administration of anti-thymocyte globulins (ATG), a set of polyclonal antibodies which lead to immune cell depletion in the recipient. It is still an open question whether ATG administration is beneficial for the patients due to the complications arising from the consecutive severe immunosuppression. So far, there are no systematic meta-analysis data about this issue.

Methods: We searched CENTRAL (the Cochrane Central Register of Controlled Trials), MEDLINE, trials registries and conference proceedings. Only randomized controlled trials comparing the prophylactic use of ATG versus no ATG were included into the analysis.

Results: Six RCTs involving 568 participants meeting the predefined selection criteria were identified by our search strategy. The primary outcome overall survival was not significantly changed by the addition of ATG for the prophylaxis of GVHD (HR $0.88 ; 95 \% \mathrm{Cl} 0.67$ to $1.15, \mathrm{P}=0.33$ ). The incidence of treatment-requiring or severe acute GVHD (grade II to IV) was significantly lower in patients who received ATG (RR 0.68; $95 \% \mathrm{Cl}$ 0.55 to $0.85, P=0.009$; NNT: 8). Also the incidence of severe acute GVHD (grade III to IV) (HR 0.53; 95\% Cl 0.33 to 0.85 , $\mathrm{P}=0.0005$; NNT: 7 ) was significantly reduced (HR $0.53 ; 95 \%$ $\mathrm{Cl} 0.33$ to $0.85, \mathrm{P}=0.0005$; NNT: 7 ), but comparable data were only available for rabbit ATG. However, pooled study results regarding the incidence of acute GVHD of all grades (I to IV) showed no significant benefit (RR $0.89 ; 95 \% \mathrm{Cl} 0.74$ to 1.06 , $\mathrm{P}=0.20$ ). Due to a lack of sufficient data, a meta-analysis concerning the incidence of chronic GVHD was not possible. Pooled data regarding the incidence of relapse were not differing significantly ( $R R 1.13 ; 95 \% \mathrm{Cl} 0.75$ to $1.68, P=0.56$ ), as well as pooled data regarding non-relapse mortality (HR 0.82; $95 \% \mathrm{Cl} 0.55$ to $1.24, \mathrm{P}=0.35$ )

Conclusions: From the currently available data no recommendation on a general use of ATG in allogeneic HSCT can be conducted. Therefore, a careful consideration of the use of ATG based on the patient's condition and risk factors of the transplantation setting should be made.
0119

Fibroblast growth factor-7 sustains thymic expression of tissue-restricted antigens during experimental acute graft-versus-host disease

S. Dertschnig (1), G. Nusspaumer (1), G. Holländer (2), W. Krenger (1)

(1)University Basel (Basel, CH); (2)University Oxford (Oxford, UK)

Outcomes of allogeneic hematopoietic stem cell transplantation (alloHSCT) depend on the successful reconstruction of T-cell adaptive immunity. This process requires in turn the de novo production of naïve $T$ cells in a competent thymus. Unfortunately, acute graft-versus-host disease (aGVHD) compromises intrathymic T-cell maturation. Impaired thymopoiesis was previously shown in murine models to be due to anti-host alloimmunity directed against components of the thymus stroma. In particular, thymic epithelial cells (TECs) - which deliver under physiological conditions the extrinsic signals required for T-cell development - are targets of disease. The systemic administration of human fibroblast growth factor 7 (Fgf7; palifermin) can, however, correct the disarray of the thymic epithelial network via its mitogenic effect on TECs and thus maintain normal T-cell development during experimental aGVHD. Fgf7's molecular and cellular mechanisms of action on thymic stromal cells are presently incompletely understood. Here we used a haploidentical murine transplantation model to study the effects of Fgf7 on medullary TEC (mTEC). A subset of these mTEC normally expresses and presents to developing $T$ cells an array of ectopic tissue-restricted antigens (TRAs). TRAs are central to the process of thymic negative selection and are partly under control of the transcription factor Aire ("autoimmune regulator"). As detected by flow cytometry and immunohistochemistry, mature mTEC cell numbers were found to be progressively diminished to almost undetectable levels in the course of aGVHD. Oligonucleotide microarray analysis revealed a contraction in the diversity of medullary TRA expression. However, Fgf7 sustained a stable population of mTEC, including the subset expressing Aire, in allogeneically transplanted mice, even if total mTEC numbers remained lower than in age-matched controls. On a whole mTEC population level, Fgf7 therapy maintained in the long-term a much more diverse array of TRA expression than in untreated mice with aGVHD. The cellular expansion of the thymic epithelial compartment in response to Fgf7 therefore conserved a crucial function of mTEC despite the continued existence of injurious donor T cells in the host thymus. Hence, a therapeutic strategy using Fgf7 may promote regeneration of a functionally competent T-cell adaptive immune system following alloHSCT.

\section{0}

Current status of acute graft-versus-host disease prophylaxis in Japan. Retrospective comparison of cyclosporine plus methotrexate versus tacrolimus plus methotrexate by donor source

R. Sakai (1), M. Taguri (2), K. Oshima (3), T. Mori (4), H. Ago (5), S. Adachi (6), S. Morita (2), S. Taniguchi (7), T. Fukuda (8), K. Ohashi(9), T.Eto(10), Y. Morishima(11), T. Nagamura-Inoue (12), H. Sakamaki (9), Y. Atsuta (13), M. Murata (13)

(1)Kanagawa Cancer Center (Yokohama, JP); (2) Yokohama City University (Yokohama, JP); (3)St. Luke International Hospital (Tokyo, JP); (4)Keio University School of Medicine (Tokyo, JP); (5)Shimane Prefectural Central Hospital (Shimane, JP); (6)Kyoto University (Kyoto, JP); (7)Toranomon Hospital (Tokyo, JP); (8)National Cancer Center Hospital (Tokyo, JP); (9)Komagome Hospital (Tokyo, JP); (10)Hamanomachi Hospital (Fukuoka, JP); (11)Aichi Cancer Center Research Institute (Nagoya, JP); (12)The University of Tokyo (Tokyo, JP); (13)Nagoya University Graduate School of Medicine (Nagoya, JP)

Background: Cyclosporine (CSP) or tacrolimus (TAC) in combination with methotrexate (MTX) are the most common 
regimens for the prophylaxis of acute graft-versus-host disease (aGvHD). To evaluate the current status of prophylaxis for aGvHD in Japan, we retrospectively analyzed data from the transplant outcome database of the Japan Society of Hematopoietic Cell Transplantation.

Methods: Data from 10,826 patients with a median age of 42 (range: $0-76 ; 85.5 \%$ aged $\geq 16$ years) who underwent allogeneic stem cell transplantation (SCT) for the first time from 2005 to 2009 were analyzed. The main underlying disease was leukemia, including acute leukemia, myelodysplastic syndrome, and chronic myelogenous leukemia $(76.4 \%)$. A total of 3,914 patients received related donor SCT (RD-SCT) and $77.9 \%$ had HLA-identical related donors. A total of 3,976 patients received unrelated bone marrow transplantation (UR-BMT) and $87.6 \%$ of them had serological HLA-matched donors. Another 2,375 patients received cord blood transplantation (CBT).

Results: Among RD-SCT patients, CSP/MTX and TAC/MTX were used for $61.2 \%$ and $23.9 \%$, respectively. Among URBMT patients, CSP/MTX was used in $19.5 \%$ and TAC/MTX in $76.3 \%$. Among CBT patients, $33.4 \%$ received CSP/MTX and $32 \%$ received TAC/MTX. We compared the cumulative incidence function (CIF) of grade II-IV aGvHD (II-IVaGvHD) at 200 days and the overall survival (OS) between patients aged $\geq 16$ years with leukemia who received CSP/MTX or TAC/MTX stratified by the donor source. Among RD-SCT patients $(n=2182)$, the CIF of II-IVaGvHD was $32.9 \%(95 \% \mathrm{Cl}: 30.7 \%-35.2 \%)$ when they received CSP/MTX and $37.4 \%(95 \% \mathrm{Cl}: 33 \%-41.8 \%)$ with TAC/MTX $(P=0.0413)$. Among UR-BMT patients $(n=3044)$, the CIF of II-IVaGvHD was $43.4 \%(95 \% \mathrm{Cl}: 39.7 \%-47.2 \%)$ in those given CSP/MTX and $39.2 \%(95 \% \mathrm{Cl}: 37.2 \%-41.2 \%)$ with TACl MTX ( $P=0.0171)$. Among patients given CBT $(n=1013)$, the CIF was $34.9 \%(95 \% \mathrm{Cl}: 31 \%-38.8 \%)$ in those given CSP/MTX and $26.3 \%$ (95\% Cl: $22.1 \%-30.4 \%$ ) with TAC/MTX ( $P=0.0007)$. On multivariate analysis, Fine and Gray's proportion-hazard anaIysis showed that TAC/MTX was correlated with a lower risk of II-IVaGvHD only in patients receiving UR-BMT (HR [95\% Cl], 0.827 [0.716-0.955], $\mathrm{P}=0.0099$ ). Multivariate Cox proportional hazards analysis showed no statistical differences of OS between the regimens regardless of the stem cell source.

Conclusion: TAC/MTX is more frequent used among UR-BMT group in Japanese stem cell transplantation and has impact on II-IVaGvHD incidence but not on OS.

\section{1}

Busulfan-based versus total body irradiation-based myelo-ablative conditioning in patients with AML: reduction of acute graft-versus-host disease incidence, mucositis and duration of hospitalisation

C. Bucher, W. Krenger, S. Dertschnig, M. Stern, S. Gerull, D. Heim, J. Halter, A. Buser, T. Lehmann, L. Graf, A. Rovo, A. Tichelli, J. Passweg

University Hospital (Basel, $\mathrm{CH}$ )

The effect of Busulfan/Cyclophosphamide (BuCy) vs. Cy(VP16)TBI on aGvHD has been evaluated in patients receiving BMT but not in patients receiving PBSCT (1). Data from 96 consecutive AML patients undergoing non-T-depleted HLAidentical PBSCT after myeloablative conditioning from Jan 2001 to Jan 2011 were included in the study. 54 Patients received 12Gy TBI $(+C y(n=26) ;+C y / V P-16(n=28))$ and 42 patients received non-targeted full dose i.v. busulfan $(\mathrm{BuCy}(\mathrm{n}=7)$ or CyBu ( $n=35$; see (2) for details). GvHD prophylaxis was with MTX/CyA. TBI and noTBI groups were comparable for all relevant pretransplant risk factors (Table 1) except for year of transplant (median 2004 vs. 2008; $p<0.001$ ) and age at transplant (41.6yrs vs. $50.0 y r s ; p<0.001$ ) which reflects the gradual introduction of Bu based regimens and increased age limits at our institution. The cumulative incidence of aGvHD at day 100 was $67.3 \%$ in the TBI and $38.6 \%$ in the noTBI $(p<0.001$; severity and organ involvement is summarized in table 1 . The median onset of aGvHD was 12(6-45) days in the TBI and 17 (7-1463) in the noTBI group $(p=0.04)$. The cumulative incidence of TRM and the relapse incidence at one year and two years respectively were 22.7 vs. $14.8 \%(p=0.96)$ and $24.2 \%$ vs. $29.6 \%(p=0.7)$. Overall survival at two years post transplant was similar $(56.8 \%$ vs. $53.7 \%, p=0.78$.). The cox regression analysis after correction for age, remission status, year of transplant and CMV status confirmed a significant aGvHD risk reduction $(R R=0.4$ $(0.18-0.90 ; p=0.028))$ for the noTBI group without influence on TRM and OS. In the TBI group, the median duration of clinical mucositis was 12 days (0-30 days), whereas in the noTBI group it was 5 days $(0-19$ days; $p<0.001)$. The cumulative median dose of morphine used for mucositis related pain was $269 \mathrm{mg}(0-3055 \mathrm{mg})$ in the TBI group vs. $36 \mathrm{mg}(0-483 \mathrm{mg})$ in the noTBI group $(p<0.001)$. Reduced morbidity resulted in a significant reduction of inhospital stay from a median of 27 days (mean $34.9(29.2-42.395 \% \mathrm{Cl}$ ) in the TBI group to a median of 21 days in the noTBI group (mean 24.5 (21.6-27.4 $95 \% \mathrm{Cl}) \mathrm{p}=0.014)$. The use of non-targeted i.v. busulfan regimen demonstrated a lower incidence of aGvHD, less toxicity and morbidity and shortened inhospital stay, making thus an attractive alternative for AML patients.

Reference:

1. Socie G. Blood. 2001;98:3569-3574. 2. Cantoni N. BMT; 2011;46:344-349.

\section{2}

Toll-like receptor 2, 4 expression and response to agonist stimulation in patients with acute and chronic graft-versus-host disease

N. Zogas (1), E. Sgouramali (1), F. lordanidis (1),

A. Papadopoulou (1), E. Tsolaki (1), I. Batsis (1), Z. Skouras

(2), I. Sakellari (1), A. Anagnostopoulos (1), E. Yannaki (1)

(1)George Papanicolaou Hospital (Thessaloniki, GR);

(2)Aristotle University (Thessaloniki, GR)

Toll-like receptors (TLRs) are evolutionary conserved receptors activated by exogenous and endogenous stimuli, having been implicated in the development of infectious and autoimmune diseases. Graft vs Host Disease (GvHD) is characterized by damage of epithelial surfaces in target organs caused by alloactivated T-cells recognizing host-tissue antigens. Intestinal bacterial breakdown products and conditioning-induced loss of gastrointestinal tract integrity result in LPS-TLR4 interaction contributing to acute GvHD whereas TLR4,9 polymorphisms have been associated with GvHD occurrence. We studied TLR2,4 expression by qRT-PCR and FCM, in peripheral blood mononuclear cells (PBMCs), lymphocyte subpopulations and target tissues as well as the activation of TLR2,4 signaling pathway by specific agonists (HKLM, LPS respectively) in PBMCs from acute and chronic GvHD patients ( $n=18, n=42$, respectively). As control groups served healthy volunteers (normal group) and allo-transplanted patients who either never developed or were cured from GvHD (no GvHD group). There was a significant upregulation of TLR2,4 expression in PBMCs of aGvHD patients, both at a transcript $(p<0,001, p<0,001)$ and a protein level $(p<0,001, p<0,001)$, whereas $c G v H D$ patients overexpressed mainly TLR2. In purified by immunomagnetic separation, T- and B-cell subpopulations, a similar TLR2 (CD3+/TLR2+: $p<0.001, C D 19+/ T L R 2+: p=0.005$ ) and TLR4 (CD3+/TLR4+: $p<0.001, C D 19+/ T L R 4+: p<0.05)$ upregulation was observed in aGvHD patients whereas there was only a trend to significance for B-cell TLR4 overexpression in cGvHD patients. TLR2,4 immunohistochemistry in skin, oral mucosa and salivary gland biopsies, showed a distinct expression pattern in aGvHD and cGvHD patients as compared to no GvHD group. In response to TLR ligand activation, a significant TLR2,4 hyporesponsiveness of PBMCs was observed by qRT-PCR and ELISA in aGvHD and cGvHD as compared to control groups: the mean fold change of TNFa and IFNb in presence vs absence of agonist per GvHD patient was significantly lower as compared to its control group counterpart [TLR2/TNFa: $p<0.01$, TLR4/TNFa: $p<0.05$, TLR4/ IFNb: $p<0.05]$. This study describes for first time in an acute and chronic GvHD setting, the PBMC and target tissue expression 


\begin{tabular}{|c|c|c|c|}
\hline & TBI & noTBI & \\
\hline Су/TBI & 26 & & \\
\hline Cy/NP-16/TBI & 28 & & \\
\hline Bu/Cy & & 7 & \\
\hline Cy/Bu & & 35 & \\
\hline $\begin{array}{l}\text { Year of transplant (median) } \\
\text { Age at transplant (yrs.) }\end{array}$ & $\begin{array}{l}2004 \\
41.6\end{array}$ & $\begin{array}{c}2008 \\
50\end{array}$ & $\begin{array}{l}p<0.001 \\
p<0.001\end{array}$ \\
\hline Cumulative incidence aGvHD in \% & 67.3 & 38.6 & $p<0.001$ \\
\hline aGvHD overall grade & 13 & 23 & $p=0.013$ \\
\hline 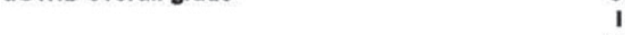 & $\boldsymbol{9}$ & 7 & \\
\hline ॥ & 16 & $\mathbf{9}$ & \\
\hline III & 13 & 2 & \\
\hline IV & 3 & 1 & \\
\hline aGvHD organ involvement & 23 & 6 & $p=0.001$ \\
\hline gastro-intestinal & 15 & 6 & $p=0.41$ \\
\hline liver & 8 & 1 & $\mathrm{p}=0.12$ \\
\hline Onset of aGvHD (median; range) & $12(6-45)$ & $17(7-1463)$ & $p=0.04$ \\
\hline Relative risk of aGvHD by day 100 , multivariate & 1 & 0.4 & $p=0.03$ \\
\hline Cumulative Incidence TRM in \% & 22.7 & 14.8 & $p=n . s$. \\
\hline Overall Survial at 2 Years & 56.8 & 53.7 & $\mathrm{p}=\mathrm{n} . \mathrm{s}$. \\
\hline & $12(0-30)$ & $6(0-12)$ & $p<0.001$ \\
\hline Cumulative dose of morphine ( $\mathrm{mg}$; median, range) & $269(0-3056)$ & $36(0-483)$ & $p<0.001$ \\
\hline Duration of hospital stay (mean, $95 \% \mathrm{Cl}$ ) & $35(29-42)$ & $25(22-27)$ & $p<0.001$ \\
\hline
\end{tabular}

of specific TLRs as well as a TLR hyporesponsiveness to agonists. Induction of TLR tolerance by repeated exposure to TLR agonists before alloHSCT might represent a novel anti-GvHD prophylaxis, worthwhile to be investigated.

\section{3}

Extracorporeal photopheresis compared to systemic immunosuppressive as second-line therapy for steroid dependent and refractory acute graft-versus-host disease: international multi-centre study

M. Robin (1), M. Jagasia (2), H. Greinix (3), E. Das-Gupta (4), R. Jacobs (2), B. Savani (2), B. Engelhardt (2), A. Kassim (2), N. Worel (3), R. Knobler (3), N. Russell (4), G. Socié (1)

(1)Hopital Saint Louis (Paris, FR); (2)Vanderbilt-Ingram Cancer Center (Nashville, US); (3)Medical University of Vienna (Vienna, AT); (4)Nottingham University Hospitals NHS Trust (Nottingham, UK)

We performed an international multicenter retrospective study of steroid refractory (SR) (progression after 3 days (d) or no response after 7d) and steroid dependent (SD) (recurrence during taper) acute graft-versus-host disease (aGVHD) comparing extracorporeal photopheresis (ECP) (Austria (AT), US, UK) to systemic immunosuppression (France) as second-line therapy (SLT) in patients (pts) undergoing transplant after January 2005. Response was assessed at end of ECP (8 weeks after ECP in AT cohort) or at 4 weeks after systemic SLT (inolimomab (anti-IL-2R)-21 pts; anti-TNF-alpha-20 pts). Table 1 outlines the characteristics of the 127 pts (ECP: AT-35, US-29, UK-22; nonECP: 41). SLT was initiated at a median of 50 (range, 8-99) $\mathrm{d}$ after transplant/DLI (10 pts). All pts received steroids prior to SLT (median of $16 \mathrm{~d}$ ). Median number of ECP treatments was 12 (range, 2-45) over a median duration of $50 \mathrm{~d}$. aGVHD response was higher in the ECP group $(73 \%$ vs. $32 \% \mathrm{P}<0.001)$ and after an ablative regimen ( $68 \%$ vs. $47 \% \mathrm{P}=0.024)$. Lower grade (gr) aGVHD $\leq 2(87 \%$ vs. $7 \% \mathrm{P}<0.001))$ and gut $(\mathrm{GI})$ aGVHD $\leq$ stage $2(68 \%$ vs. $43 \% \mathrm{P}=0.008)$ at SLT-onset were associated with a higher response rate to SLT. aGVHD gr $(\leq v s .>2)$ (adjusted for type of SLT, regimen intensity) was an independent predictor of response (OR=72.2, 95\% Cl 18-288.4 $\mathrm{P}<0.001)$. The median follow-up of surviving pts after SLT is 29.7 (range 2.1-78.3) months $(\mathrm{m})$ (ECP-33.7 $\mathrm{m}$ vs. non-ECP$17.9 \mathrm{~m}, \mathrm{P}=0.005)$. Median and 2 -yr survival for all patients is $20(95 \% \mathrm{Cl} 0-43) \mathrm{m}$ and $47 \%$, respectively. Use of ECP (not reached (NR) vs. $4.9 \mathrm{~m} \mathrm{P}<0.001$ ), ablative regimen (NR vs. $6.3 \mathrm{~m} \mathrm{P}=0.006$ ), and aGVHD gr $\leq 2$ at onset (NR vs. $1.5 \mathrm{~m}$,
$P<0.001)$ were associated with superior survival. Responders to SLT compared to non-responders had a superior survival (NR vs. $1.7 \mathrm{~m} \mathrm{P}<0.001$ ) with a 70\% 2-yr survival in responders. aGVHD gr>2 at onset of SLT (adjusted for regimen intensity, type of SLT) was associated with inferior survival (HR=8.66, $95 \% \mathrm{Cl}$ 4.72-15.8 $\mathrm{P}<0.001)$. In pts with SR/SD aGVHD $\leq$ gr2 at onset of SLT, non-ECP SLT (adjusted for regimen intensity) was associated with an inferior survival $(\mathrm{HR}=2.53,95 \% \mathrm{Cl}$ 1.04-6.11 $\mathrm{P}=0.039$ ). Although limited by its retrospective nature and patient selection bias, this international multicenter study suggests that ECP is an effective SLT therapy for SD or SR aGVHD and maybe superior to non-ECP intervention.

\section{4}

Multicentre, prospective and blind validation of acute graft-versus-host-disease-specific proteomic patterns in a large patient cohort: prediction of acute GvHD grade III and IV

E. Weissinger (1), I. Hamwi (1), C. Dobbelstein (1), M. Schleuning (2), J. Metzger (3), L. Hambach (1), E. Holler (4), H. Greinix (5), U. Borchert (1), M. Morgan (1), J. Franke (3), S. Buchholz (1), D. Wolf (4), E. Dammann (1), S. Ehrlich (1), H. Diedrich (1), M. Stadler (1), H. Mischak (3), J. Krauter (1), A. Ganser (1)

(1)Hannover Medical School (Hannover, DE); (2)Diagnostic Wiesbaden (Wiesbaden, DE); (3)mosaiques-diagnostics GmbH (Hannover, DE); (4)University of Regensburg (Regensburg, $D E) ;(5) M e d i c a l$ University of Vienna (Vienna, AT)

Allogeneic hematopoietic stem cell transplantation (allo-HSCT) is the only curative treatment of hematologic malignancies and hematopoietic failure syndromes in adult patients, but compromised by acute graft-versus-host disease (aGvHD). aGvHD-specific urinary peptide biomarkers were combined in a classifier enabling examiner-independent, unbiased diagnosis of aGvHD as reported earlier, based on urine from 141 patients transplanted in 4 transplant centers. Here, additional 315 patients transplanted between 2006 and 2010 were prospectively monitored with this aGvHD-specific proteomic classifier. The median age at transplantation was 48 years, ranging from 17 to 71 . The majority of the patients were transplanted for acute leukemias $(n=169)$ followed by chronic diseases (MDS, MPS, $\mathrm{CML}, \mathrm{n}=115), 21$ lymphomas and 10 non-malignant disease. Complete remission (CR) at transplantation was achieve in 144 patients, while 146 were not in CR. Unrelated donors were found for 203 patients. Reduced intensity conditioning was 


\begin{tabular}{|c|c|c|}
\hline & $\operatorname{ECP}(\mathrm{N}=86)(\%)$ & Non-ECP $(\mathrm{N}=41)(\%)$ \\
\hline \multicolumn{3}{|c|}{ Pre-transplant charactenistics } \\
\hline Age, median (years), range & $46.8(17-66.8)$ & $44(5-64)$ \\
\hline \multicolumn{3}{|l|}{ Gender, recipient } \\
\hline Male & $48(56)$ & $25(61)$ \\
\hline Female & $38(44)$ & $16(39)$ \\
\hline \multicolumn{3}{|l|}{ Donortype } \\
\hline Related & $16(19)$ & $9(22)$ \\
\hline Unrelated & $70(81)$ & $32(78)$ \\
\hline \multicolumn{3}{|l|}{ Stem cell source } \\
\hline Marrow & $12(14)$ & $7(17)$ \\
\hline Peripheral blood & $67(78)$ & $28(68)$ \\
\hline Umbilical Cord & $7(8)$ & $6(15)$ \\
\hline \multicolumn{3}{|l|}{ Regimen } \\
\hline Ablative & $56(65)$ & $20(49)$ \\
\hline RICINMA & $30(35)$ & $21(51)$ \\
\hline \multicolumn{3}{|l|}{ HLA match } \\
\hline $10 / 10$ & $68(79)$ & $31(76)$ \\
\hline Other & $18(21)$ & $10(24)$ \\
\hline \multicolumn{3}{|l|}{ in-vivo T-cell depletion } \\
\hline None & $68(79)$ & $37(90)$ \\
\hline ATGICampath & $18(21)$ & $4(10)$ \\
\hline \multicolumn{3}{|c|}{ aGVHD characteristics } \\
\hline \multicolumn{3}{|l|}{ indication for sakage therapy } \\
\hline Steroid-refractory & $57(66)$ & $41(100)$ \\
\hline Steroid dependent & $24(28)$ & - \\
\hline \multicolumn{3}{|l|}{$\begin{array}{c}\text { aGVHD grade'stage at onset of } \\
\text { salvage therapy }\end{array}$} \\
\hline Grade 3-4 & $38(44)$ & $22(53)$ \\
\hline Stage 3-4-skin & $21(26)$ & $20(49)$ \\
\hline Stage 3-4 -gut & $29(34)$ & $15(37)$ \\
\hline Stage 3-4 -liver & $15(18)$ & $15(37)$ \\
\hline 3 organ involvement & $17(20)$ & $3(5)$ \\
\hline \multicolumn{3}{|l|}{ Steroid dose $(\mathrm{mg} / \mathrm{kg})$ at sanage } \\
\hline 1 & $27(31)$ & - \\
\hline 2 & $57(66)$ & $37(90)$ \\
\hline other & $2(2.4)$ & $4(10)$ \\
\hline \multicolumn{3}{|c|}{ Response } \\
\hline Overall response & $62(72)$ & $13(32)$ \\
\hline Complete response & $53(62)$ & $8(20)$ \\
\hline Partial response & $9(11)$ & $5(12)$ \\
\hline \multicolumn{3}{|c|}{ Survival } \\
\hline Dead & $37(43)$ & $31(76)$ \\
\hline Alive & $49(57)$ & $10(24)$ \\
\hline \multicolumn{3}{|l|}{ Cause of death } \\
\hline GVHD & 22 & 25 \\
\hline Disease relapse & 7 & - \\
\hline Infection & 7 & 2 \\
\hline Other & 1 & 4 \\
\hline Survival, median (months) $(95 \% \mathrm{Cl})$ & Not reached & $4.9(2.8-7.1)$ \\
\hline 2-y survival & $58 \%$ & $12 \%$ \\
\hline Steroid refractory & $N=57$ & $N=41$ \\
\hline Survival, median (months) $(95 \% \mathrm{Cl})$ & Not reached & $4.9(2.8-7.1)$ \\
\hline
\end{tabular}

applied to 224 patients. GvHD-prophylaxis was a combination of CSA and MTX or MMF in 279 patients, 22 had other GvHDprophylaxis, 3 had none, about $80 \%$ received additional antithymocyte globulin prior to transplant. 10 recipients were T-cell depleted. Applying the previously established aGvHD classifier to the blinded, prospectively collected urine samples allowed correct classification of patients with aGvHD about 10 days ( 1 to 21 days) prior to clinical diagnosis with a sensitivity of $80.26 \%$ [Cl 95\%:74.6-85.28] and 75.5\% specificity [Cl 95\%: $72.2-$ 78.5]. aGvHD grade III and IV development could be predicted 21 days prior to clinical diagnosis with accuracy greater $85 \%$, distinguishing patients with aGvHD grade I and II from those with higher grade complications. We identified peptides from collagen, albumin, beta2 microglobulin and CD99 as biomarkers, indicating significant disturbance in collagen metabolism and T-cell activation. Safety and feasibility of a proteomic-classifier-driven pre-emptive steroid treatment was assessed: 49 patients received pre-emptive treatment within $72 \mathrm{~h}$ upon proteomic results positive for aGvHD. Pre-emptive therapy did not lead to increased relapse rates or infections. The results indicate that proteomic screening of urine after allo-HSCT predicts aGvHD development accurately at least 10 days prior to clinical diagnosis and allows prediction of developing aGvHD grades III and IV 3 weeks prior to clinical manifestation. 


\section{Acute Leukaemia 1}

\section{5}

Cost-effectiveness of double-unit cord blood transplantation versus single-unit cord blood transplantation in adult patients with acute leukaemia in France

M. Labopin (1), A. Ruggeri (2), N. Taright (3), F. Garnier (4), C. Faucher (4), A. Le Coroller (5), N. Milpied (6), D. Blaise (5), M. Badoglio (7), M. Mohty (7), A. Buzyn (2), F. Volt (2), E. Gluckman (2), N. Gorin (1), V. Rocha (2)

(1)Hôpital St Antoine (Paris, FR); (2)Eurocord (Paris, FR); (3)APHP (Paris, FR); (4)ABM (Paris, FR); (5)Institut PaoliCalmettes (Marseille, FR); (6)SFGM-TC (Bordeaux, FR); (7)ALWP (Paris, FR)

Single (s) or double (d) cord blood transplantation (CBT) are valid treatment for hematologic diseases. One limitation of using dCBT is the cost of the 2 units, but comparison of the total cost of the 2 procedures has not yet been investigated.

We analyzed costs-effectiveness of dCBT compared to sCBT in 134 pts with acute leukemia. CBT was performed in France from 2001-2009. All hospital costs were estimated from donor search to 1-year after CBT, according to the French public health system. A Markov decision analysis model was used to calculate the QALY (quality-adjusted life years) and cost-effectiveness ratio (ICER). For cost-effectiveness analysis, reduced intensity conditioning (RIC) and myeloablative conditioning (MAC) were analyzed separately. Forty pts were transplanted for ALL and 94 for AML, in CR1. Sixty one pts received a SCBT and 73 a dCBT. Seventy nine pts had a RIC and 55 a MAC. Neutrophil recovery was achieved in 115 pts in a median time of 23 days. Neutrophil recovery was not different after SCBT or dCBT. dCBT was associated with higher acute GVHD grade II-IV: $56 \%$ vs $30 \%$ for sCBT, $p=0.003$. At day- $100,53 \%$ of pts had CMV reactivation (37\% after SCBT and $71 \%$ after dCBT $\mathrm{p}=0.01$ ), $45 \%$ had viral infection other than CMV and $49 \%$ had bacterial infection. Fifteen pts (11\%) had a 2nd transplant, 6 for graft failure (4 in sCBT group and 2in dCBT group) and 9 for relapse ( 6 in sCBT group and 3 in dCBT group). Cumulative incidence of relapse at 2-year was lower after dCBT: $29 \%$ vs to $42 \%$ after $\mathrm{sCBT}(\mathrm{p}=0.04)$. No difference was observed for NRM and chronic GVHD. Survival at 2-year as $40 \%$ vs $58 \%$ after $s C B T$ and dCBT $(p=0.04)$. Leukemia-free survival at 2-year was $30 \%$ in sCBT vs $49 \%$ in dCBT $(p=0.09)$. The mean cost for donor identification and UCB acquisition was $28.164 €$ for sCBT and $48.929 €$ for dCBT. The estimated costs within 1 year after RIC-sCBT was $133.790 €$ and it was $211.735 €$ after MACsCBT. The estimated cost was $180.549 €$ after RIC-dCBT and $205.375 €$, after MAC-dCBT. Table 1 summarizes costs by type of graft and conditioning. In the MAC group, dCBT was associated with lower cost (minus 13.554€) and better effectiveness (plus 0,53 QALY). The cost per QALY obtained after RIC-dCBT compared with SCBT was 91.199€. In France, dCBT is associated with higher incidence of acute GVHD, lower relapse and better survival in adults transplanted for acute leukemia. With MAC, dCBT is the best option and the cost per QALY obtained for dCBT when using RIC is acceptable.

\section{6}

Maternal microchimerism in CB mediates a graft-versusleukaemia effect in unrelated $C B$ graft recipients J. van Rood (1), A. Scaradavou (2), C.E. Stevens (3) (1)Europdonor Foundation (Leiden, NL); (2)New York Blood Center (New York, US); (3)New York Blood Center, retired (New York, US)

During pregnancy maternal $\mathrm{B}$ and $\mathrm{T}$ cells may become primed against fetal HLA and minor Histocompatibility Antigens ( $\mathrm{mHA}$ ) inherited paternal antigens (IPA). Anti-IPA immunity accounts for the lower relapse rates in maternal haplo-identical hemato-

[0125] Table 1: Estimated costs from donor search to 1 year after transplantation for single CBT, double CBT and type of conditioning regimen (MAC or RIC)

\begin{tabular}{|c|c|c|c|c|}
\hline & 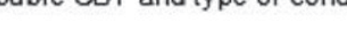 & & sCBT & dCBT \\
\hline & CB unit search & & $28164 €$ & $48929 €$ \\
\hline MAC & Initial hospitalisation & Mean duration (d) & 66 & 65 \\
\hline & & Mean cost & $137.757 €$ & $131.773 €$ \\
\hline & Outpatient visits & Number of days & 9 & 11 \\
\hline & & Mean cost & $7.788 €$ & $9.223 €$ \\
\hline & Further hos pita lisations & Mean duration $(d)$ & 50 & 23 \\
\hline & & Mean cost & $38.026 €$ & $15.449 €$ \\
\hline & Total mean cost within 1 year & & $211.735 €$ & $205.374 €$ \\
\hline RIC & Initial hospitalisation & $\begin{array}{l}\text { Mean duration (d) } \\
\text { Mean cost }\end{array}$ & $\begin{array}{c}29 \\
58.621 €\end{array}$ & $\begin{array}{c}48 \\
96.335 €\end{array}$ \\
\hline & Outpatient visits & Number of days & 21 & 23 \\
\hline & & Mean cost & $17.870 €$ & $19.366 €$ \\
\hline & Further hos pita lis ations & Mean duration (d) & 40 & 21 \\
\hline & & Mean cost & $29.135 €$ & $15.918 €$ \\
\hline & Total mean cost within 1 year & & $133.790 €$ & $180.549 €$ \\
\hline
\end{tabular}



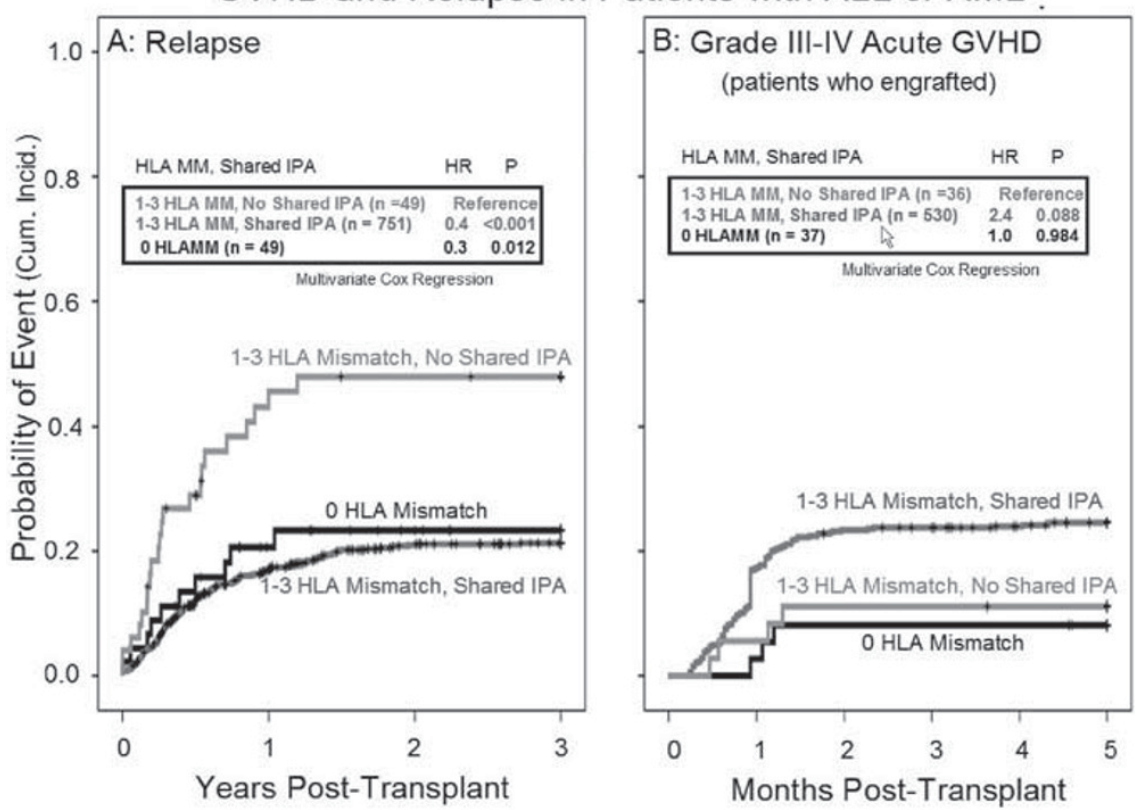

poietic stem cell (HSC) transplants compared to paternal ones (Stern, et al. Blood 2008). We hypothesized that CB graft recipients who share the same antigen(s) as their CB donor IPAs might have a lower incidence of relapse due to the presence of primed maternal cells than recipients who have no antigens shared with their CB IPAs.

Methods: We carried out a retrospective, observational study in 1,155 patients with hematological malignancies who received single unit CB grafts from NYBC between 1993-2006, including 453 with ALL, 392 with AML and 310 with CML, MDS and lymphomas. Primary study endpoints were relapse and grade III-IV acute GVHD; secondary endpoints were engraftment, mortality and treatment failure relapse or death). Maternal HLA typing was available, permitting inferred identification of CB IPAs at HLA-A, -B (intermediate resolution) and DRB1 (high resolution). Patients were classified by whether they shared the same antigen(s) as the CB IPAs or not, as well as by the number of HLA mismatches, mismatch direction and match to non-inherited maternal HLA (NIMA.

Results: Among 1,094 patients given HLA mismatched CB units, 1,030 shared antigens with one or more CB IPA targets and 64 $(6 \%)$ had no shared IPA target. Transplants with a shared IPA target had a significantly lower incidence of relapse during the first 3 years post-transplantation compared to those that had no shared IPA target. This effect was detected in both ALL and AML (combined hazard ratio $=0.4$ in a multivariate analysis with other risk factors, $P<0.001$. Figure $1 \mathrm{~A}$ ) but not in patients with other malignancies. The lower relapse rate was strongest in patients with $1 \mathrm{HLA}$ mismatch $(\mathrm{HR}=0.1, \mathrm{P}<0.001)$ and was reflected in a lower treatment failure rate in this group $(\mathrm{HR}=0.5, \mathrm{P}=0.012)$. Acute GVHD in patients with a shared IPA target was increased but not significantly so (Figure 1B). Maternal HLA match to the recipient (a control for possible effects of unprimed maternal cells) was not associated with relapse risk.

We conclude that microchimeric IPA-primed maternal cells in CB can after HSCT reduce relapse in patients with ALL and $\mathrm{AML}$ and might play a role in cancer surveillance in general.
0127

Second allogeneic HSCT in patients with acute leukaemia relapsed after a first allogeneic transplantation: a retrospective GITMO study

A. Dominietto, A. Bacigalupo, B. Bruno, M. Zecca, M. Falda, P. Di Bartolomeo, W. Arcese, A. Bosi, F. Fagioli, E. lannitto, C. Favre, A. Rambaldi, A. Pession, G. Bandini, F. Ciceri oh behalf of GITMO

Background: Despite the reduction of transplant related mortality (TRM) leukemia relapse after allogeneic hematopoietic stem cell transplantation (HSCT) remains unchanged over the past two decades.

Aim of the study We retrospective analyzed the outcome of 289 patients with acute leukemia relapsed after allogeneic HSCT who received a second allogeneic transplantation and evaluated the prognostic value of the EBMT risk score as predictor of survival.

Patients and Methods: From January 2005 to December 2010 170 patients with acute myeloid leukemia and 119 with acute lymphoblastic leukemia underwent to a second allogeneic HSCT in 41 Italian Bone Marrow Transplant centers. Patients characteristics are listed in Table 1.

Results: We used the EBMT risk score based on 5 pre-transplant variables, age ( $<3030 \mathrm{yy}$ ), disease phase (early vs advanced), interval time from 1st to 2nd transplant (<> 1 year), donor type (SIB vs ALT) and donor-recipient sex mismatched ( $F->M$ vs others) In multivariate Cox analysis negative predictive factors on overall survival (OS) were: interval time from 1st to 2 nd transplant $<1$ year (RR 0.6, 95\% Cl 0.4-0.9, p<0.01), age $>30$ yy (RR $1.4,95 \%$ Cl 1-1.9, $p<0.04$ ), advanced disease phase (RR 1.4, 95\% Cl 1-2, p<0.03) and alternative donor (RR 1.7, $95 \% \mathrm{Cl} 1.2-2.3, \mathrm{p}<0.002$ ). Disease phase and donor type were favorable predictive factors on non-relapse mortality (NRM) in multivariate Cox analysis. We also analyzed the impact on the outcome of a different donor type for second allogeneic HSCT and we observed no effect both on OS and NRM in multivariate analysis.

We therefore identified three groups of patients: group A score $0-2(n=117)$, group $B$ score $3(n=81)$, group C score $4-5(n=51)$. The actuarial 5yy OS according to group A (Low risk), group B (Intermediate) and group C (High risk) was $26 \%$ vs $19 \%$ vs $14 \%$ respectively, $p<0.001$ (Figure 1). The cumulative incidence of 
NRM according to Low, Intermediate and High risk group was $19 \%$ vs $26 \%$ vs $35 \%$ respectively, $p<0.01$.

Conclusions: This study confirms that the EBMT score is a useful predictor of outcome for patients with acute leukemia receiving a second allogeneic transplant because of a leukemia relapse. The 5 year actuarial ranges from $26 \%$ to $14 \%$, and it may thus be possible to select patients who will most benefit from a second transplant. In our series changing donor had no impact on the outcome.

Table 1. Patient characteristics

\begin{tabular}{|c|c|}
\hline Number of patients & 289 \\
\hline Median Age (range) & $32(2-68)$ yy \\
\hline Donor Type & $\mathbf{n}$ \\
\hline HLA id sib. & 128 \\
\hline Alternative & 161 \\
\hline Stem Cell Source & \\
\hline BM & 83 \\
\hline PBSC & 179 \\
\hline$B M+P B S C$ & 3 \\
\hline $\mathrm{CB}$ & 22 \\
\hline Sex mismatched & \\
\hline F $\rightarrow$ Mothers & $71 / 184$ \\
\hline Disease Phase & \\
\hline Early/Advanced & $93 / 174$ \\
\hline Conditioning & \\
\hline Myeloablative & 143 \\
\hline Non Myeloablative & 96 \\
\hline GvHD prophylaxis & \\
\hline CYA+MTX & 215 \\
\hline Tcell depl & 26 \\
\hline $\begin{array}{l}\text { No proph. } \\
\text { Different Donor Type } 2^{\text {nd }} \mathrm{H} \mathrm{SCT}\end{array}$ & 27 \\
\hline YesiNo & $90 / 199$ \\
\hline Median in terval $1^{\text {st }}-2^{\text {nd }}$ HSCT (range) & $341(21-2997) d d$ \\
\hline Median days of FUP (range) & $158(4-1849)$ \\
\hline
\end{tabular}

Figure 1. Actuarial 5yy OS according to Low, Intermediate or High risk score

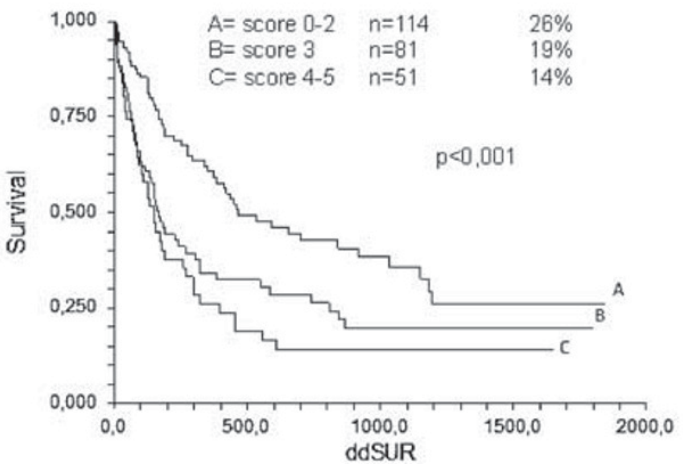

0128

The influence of monosomal karyotype on survival in patients with acute myeloid leukaemia after allogeneic haematopoietic stem cell transplantation: a retrospective survey on behalf of the Acute Leukaemia Working Party of the EBMT

A. V.M. Brands-Nijenhuis, M. Labopin, H.C. Schouten, L. Volin, G. Socie, J.J. Cornelissen, M. Attal, R. Tabrizi, J. Esteve, M. Mohty on behalf of the Acute Leukemia Working Party of the EBMT, molecular subcommittee

Introduction: Monosomal karyotype (MK) has been shown to be associated with a very poor prognosis in AML patients
(Breems, 2008). Whether allogeneic hematopoietic stem cell transplantation (alloHSCT) performed in an early phase can overcome the adverse prognosis in this cytogenetic patient category is currently unknown. To address this issue we performed a retrospective analysis on data from the registry of the EBMT among patients with primary AML who underwent alloHSCT in CR1.

Patients and Methods: A total of 4119 patients with primary AML and known cytogenetic abnormalities at diagnosis that underwent alloHSCT in CR1 were included in the analysis. Survival curves were calculated with Kaplan-Meier method. Log rank test and Cox regression analysis were used to determine statistical significance.

Results: Median follow-up was 24 months (range 2-374). Overall, 171 patients (4.2\%) fulfilled criteria for MK and 297 patients $(7.2 \%)$ for complex karyotype (CK), with 115 patients fulfilling both conditions (MK and CK). Both the presence of a MK (2-yr OS: $35.5 \%$ versus $63.2 \%, p<0.0001)$ and CK $(2-y r$ OS: $48.8 \%$ versus $61.9 \%, p<0.0001$ ) were associated with a poorer outcome when compared with the remaining cytogenetics subtypes. Given the significant overlap between both categories, we further analyzed their prognostic impact after defining four subgroups of patients: MK but not CK (56 patients; MK+CK-), no MK but CK (180 patients; MK-CK+), MK and CK (115 patients; $\mathrm{MK}+\mathrm{CK}+$ ), and patients without either MK or CK (MK-CK-). Outcome of the MK-CK- subgroup did not differ according to cytogenetics. Patients harboring a MK, regardless concomitant presence of a CK, presented with a poorer OS after alloHSCT (2-yr OS: $31.7-43.0 \%$ versus $61.1 \%, p<0.0001)$. On the contrary patients with a CK but not MK showed a similar outcome than MK-CK- (2-yr OS: $61.1 \%$ versus $63.3 \%, p=0.170)$. Moreover, multivariate analysis confirmed the independent negative impact of MK (HR:1.90, range 1.5-2.4; $p<0.0001)$ together with age, interval diagnosis-transplant, AML subtype, WBC at diagnosis, T-cell depletion, number of induction cycles and use of TBI during conditioning, whereas the presence of a CK did not retain its negative prognostic value.

Conclusion These results indicate that $\mathrm{MK}$ is a better indicator for poor outcome than CK after alloHSCT in patients with primary AML in CR1. Nonetheless, the potential curative role of alloHSCT for a subset of patients with MK should be further investigated.

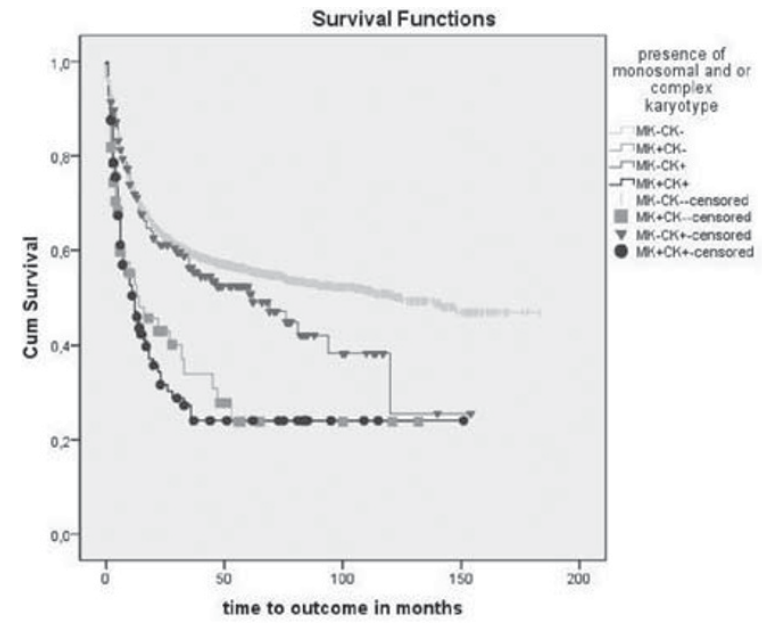




\section{9}

The CMV serostatus of the recipient and the donor is still an important prognostic factor in acute leukaemia patients after allogeneic stem cell transplantation in the era of pre-emptive therapy of CMV infection. A survey on behalf of the ALWP of EBMT

M. Schmidt-Hieber (1), M. Labopin (2), D. Beelen (3), L. Volin (4), G. Ehninger (5), J. Finke (6), G. Socié (7), R. Schwerdtfeger (8), N. Kröger (9), A. Ganser (10), D. Niederwieser (11), E. Polge (2), I. Blau (1), M. Mohty (2)

(1)HELIOS Klinikum Berlin Buch/Charite-Campus Benjamin Franklin (Berlin, DE); (2)EBMT Data \& Study Office (Paris, FR); (3)University Hospital Essen (Essen, DE); (4)Helsinki University Central Hospital (Helsinki, Fl); (5)Universitätsklinikum Dresden (Dresden, DE); (6)University of Freiburg (Freiburg, DE); (7)Hopital St. Louis (Paris, FR); (8)Deutsche Klinik für Diagnostik (Wiesbaden, DE); (9)University Hospital Eppendorf (Hamburg, DE); (10)Hannover Medical University (Hannover, DE); (11)University Hospital Leipzig (Leipzig, DE)

Objectives: There are no systematic data on the prognostic impact of the recipient's and donor's cytomegalovirus (CMV) serostatus focused on the era of preemptive therapy of CMV infection in patients after allogeneic stem cell transplantation (allo-SCT)

Methods: We analyzed 16628 adult de novo acute leukemia patients documented in the registry of the European Bone Marrow Transplantation (EBMT) group allografted between 1998 and 2009 (cohort I). Additionally, 97 acute leukemia patients who underwent allo-SCT (University Medicine Berlin; Germany) were analyzed in more detail including kinetics of the hematopoietic chimerism and CMV infection (cohort II).

Results: CMV seronegative recipients (R-CMV-) allografted from a CMV seronegative donor (D-CMV-) had a significant $(p<0.001)$ better median overall survival (OS) (56 months) and leukemia-free survival (LFS) (49 months) than R-CMV-I $\mathrm{D}-\mathrm{CMV}+$ (49 and 44 months), R-CMV+/D-CMV- (49 and 43 months) and R-CMV+/D-CMV+ (51 and 45 months) cohort I cases, respectively. The recipient/donor CMV serostatus remained as a significant prognostic factor in a multivariate analysis. The negative impact of the recipient's and/or the donor's CMV seropositivity was due to both an increased treatment-related and relapse-related mortality and strongest in patients with a matched unrelated donor. Conversely, the CMV serostatus had no significant impact on the occurrence of graft-versus-host disease or graft failure.

The CMV infection risk in comparison to R-CMV-/D-CMV+ cases was 3-fold for R-CMV+/D-CMV+, 4-fold for R-CMV+/ D-CMV- and 9-fold for R-CMV-/D-CMV+ cohort II patients. Recipient's CMV seropositivity was also in this cohort associated with an inferior OS (66\% vs. $35 \% 3$ years after allo-SCT; $p=0.03$ ) and, even more interestingly, this impact remained if analyzing only patients who did never develop a CMV reactivation until the last follow-up. Finally, recipients seropositivity was associated with a lower rate of complete day +30 chimerism $(68 \%$ vs. $92 \%, p=0.02)$ without influencing time to engraftment or immune reconstitution kinetics of $\mathrm{CD} 4+\mathrm{T}$ cells, CD8+ $\mathrm{T}$ cells and NK cells.

Conclusion: The recipient/donor CMV serostatus remains an important prognostic factor despite the increasing use of sophisticated techniques to diagnose and treat CMV infections in acute leukemia patients after allo-SCT. Interestingly, this observation seems to be mainly explained by indirect virus effects and not a CMV reactivation itself.

\section{0}

Evaluation of bone marrow cytomorphology after allogeneic stem cell transplantation in patients with acute myeloid leukaemia

M. Christopeit (1), K. Miersch (2), E. Klyuchnikov (2), T. Haferlach (3), M. Binder (4), T. Zabelina (2), F. Ayuk (2), P. Schafhausen (4), A. Zander (2), C. Bokemeyer (4), N. Kröger (2), U. Bacher (2)

(1)Albert Einstein College of Medicine (New York, US); (2)Clinic for Stem Cell Transplantation (Hamburg, DE); (3)MLL Munich Leukemia Laboratory (Munich, DE); (4)University Cancer Center Hamburg (Hamburg, DE)

Objectives: In patients with AML, bone marrow (BM) cytomorphology allows monitoring the remission status post-transplant, but the impact of other morphologic features such as dysplasia or cellularity in this specific setting remains unclear.

Methods: We analysed the frequency and the prognostic impact of these parameters in stem cell recipients with AML. 112 patients (60 males, 52 females; $17-72$ yrs) received unrelated/related allogeneic HSCT following reduced/myeloablative conditioning resulting in blast clearance $\leq 5 \%$. BM cytomorphology was performed at days $+30(\mathrm{~d} 30)$ and/or +100 (d100) posttransplant. Following the criteria of Goasguen et al. (1992), thresholds of $10 \%, 20 \%$, and $50 \%$ of dysplastic cells were used to define any hematopoietic lineage as "dysplastic". Overall BM cellularity and cellularity in granulopoiesis (GP), erythropoiesis (EP), and megakaryopoiesis (MP) were analysed performing age-related adjustment.

Results: Dysplasia in $\geq 10 \%$ of cells was frequent in all hematopoietic lineages on d30 (MP: $21 / 44 ; 47.7 \%$ pts evaluable in this lineage; EP: $18 / 52 ; 34.6 \%$; GP: $15 / 60 ; 25.0 \%$ ) and also on d100. On d30, normal overall BM cellularity was seen in only $34 / 75$ pts $(45.3 \%)$ with available morphology, while cellularity was increased in $13(17.3 \%)$ pts and reduced in $28(37.3 \%)$. Also on d100, aberrant cellularity was frequent (increased: $n=4,6.5 \%$; reduced: $n=24,38.7 \%$ ). Relapses were less frequent in pts with normal BM cellularity on d30 (7/34; 20.6\%) than in those with reduced $(9 / 28 ; 32.1 \%)$ or increased cellularity $(10 / 13 ; 76.9 \% ; p=0.001)$. Reduced overall cellularity on $d 30$ significantly correlated with inferior 2-year OS rates as compared to increased or normal cellularity $(31.4 \%$ vs. $44.0 \%$ vs. $59 \% ; p=0.009$ ). Reduced cellularity separately in GP, EP, and $\mathrm{MP}$ on d30 also correlated with inferior OS. Competing risk factor analysis revealed that pts with increased cellularity on d30 had a cumulative relapse incidence of $62 \%(95 \% \mathrm{Cl} 35 \%-89 \%)$ with a HR of $6.68(p=0.00014)$. Pts with reduced cellularity had a NRM of $36 \%(95 \% \mathrm{Cl} 18 \%-54 \%)$ with a HR of $2.6(p=0.006)$. In contrast, dysplasia in at least $10 \%, 20 \%$, or $50 \%$ of cells on days +30 and +100 and cellularity on d100 did not correlate with survival/relapses.

Conclusion: Aberrant cellularity is frequent in the post-transplant period and seems to represent an additional prognostic parameter for stem cell recipients with AML. In contrast, dysplasia seems to be an unspecific phenomenon post-transplant.

\section{1}

Leukaemia relapse after allogeneic transplants for

AML: predictive role of WT1 expression

S. Pozzi, A. Dominietto, S. Geroldi, F. Bertolotti, C. Di Grazia, S. Luchetti, T. Lamparelli, F. Gualandi, A. Ibatici, S. Bregante, M.T. van Lint, A. Raiola, F. Frassoni, A. Bacigalupo

S.Martino's Hospital (Genoa, IT)

In this study we test the predictive value of WT1 expression on leukemia relapse in 131 consecutive patients with acute myeloid leukaemia (AML) patients undergoing an allogeneic hemopoietic stem cell transplant (HSCT). WT1 expression was assessed before and after HSCT, and expressed as copy numbers/ $10^{4} \mathrm{abl} ; 72$ patients were in first complete remission (CR1), 28 in second or subsequent tremission and 27 had active disease at the time of transplant; the median age was 
44 years (15-69); 87 patients received a myeloabltive conditioning, and 34 a reduced intensity regimen. End points of the study were cumulative incidence of transplant related mortality (TRM), relapse related death (RRD) and actuarial overall survival (OS).

Pre-transplant WT1. The median pre-transplant WT1 level in marrow cells, was 100 copies: TRM was $12 \%$ vs $13 \%$ for patients with pre-transplant WT1 levels $<>>100(p=0.8)$; RRD was $23 \%$ vs $47 \%(p=0.004)$ and OS $65 \%$ vs $40 \%(p=0.004)$.

Post-transplant WT1. On day+30 post transplant patients were again re-evaluated: TRM was $12 \%$ vs $10 \%$ for post-transplant WT1 levels $<>>100$ (ns); RRD was $29 \%$ vs $53 \%(p=0.01)$ and OS was $58 \%$ vs $37 \% \quad(p=0.03)$.

Pre+Post Transplant WT1. When combining WT1 expression pre and post transplant we could identify 2 groups: group A (patients with WT1 $<100$ either pre and/or post transplant), and group B (patients with WT1 $>100$ pre and post transplant). The RRD is $26 \%$ in group $A$ and $70 \%$ in group $B(p<0.00001)$.

We then asked the question: can we identify patients in group A who will relapse.

To answer this question we looked at WT1 expression on day +60 , and looked at the difference between WT1 expression on day +60 and day +30 . We found that when WT1 expression was higher on day +60 than on day $+30(n=36)$ patients $)$ then the risk of RRD was $39 \%$ compared to $21 \%$ if WT1 expression on day +60 was not higher as compared to day+30 $(n=72)(p=0.04)$. In conclusion WT1 expression pre and post-HSCT identifies 3 groups of patients: low risk -patients with low WT1 expression pre or post HSCT day+30 and no increase in WT1 on day+60 $(n=72 ;$ RRD $21 \%)$, the intermediate risk with WT1 low expression but increase on day $+60(n=36$; RRD $39 \%)$ and the high risk patients with high WT1 pre and post trasnsplant $(n=26$; RRD $70 \%$ ). High risk and especially intermediate risk patients could be considered for early pre-emptive therapy.

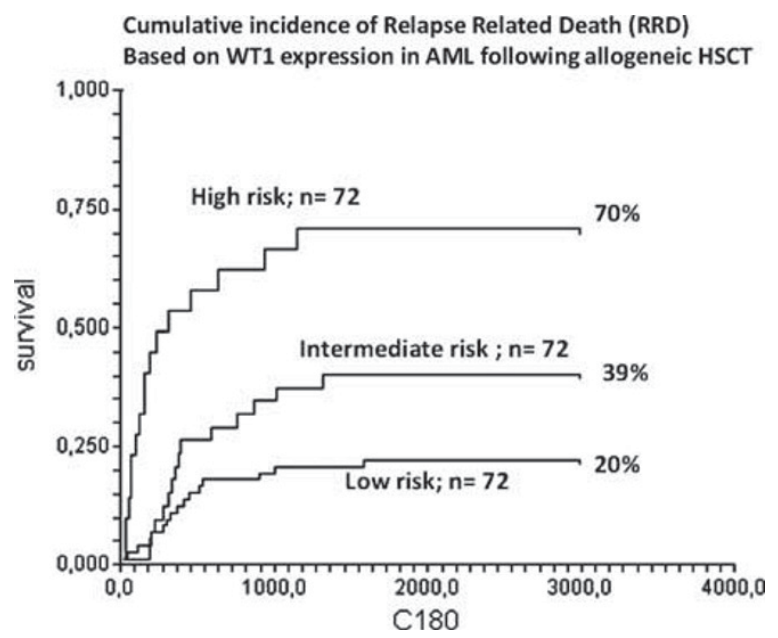

\section{Stem Cell Mobilization and Graft Engineering}

\section{2}

Transplantation of TCRalpha/beta/CD19 depleted stem cells from haploidentical donors: robust engraftment and rapid immune reconstitution in children with high-risk leukaemia

F. Locatelli (1), P. Lang (2), M.E. Bernardo (1), T. Feuchtinger (2), S. Rutella (1), A. Bertaina (1), M. Schumm (2), H.M. Teltschik (2), A.S. Schulz (3), A. Moretta (4), M. Caniglia (1), F. Zinno (1), L. Moretta (4), R. Handgretinger (2)

(1)IRCCS Bambino Gesu' Children's Hospital (Rome, IT); (2)Children's University Hospital (Tübingen, DE); (3)Children's University Hospital (UIm, DE); (4)University of Genoa (Genoa, IT)

In haploidentical HSCT, in vitro T-cell depletion of the graft is an effective method to prevent GvHD. We investigated a new T-cell depletion method (using the Clini-MACS system) which removes alpha/beta T cells and B cells, while retaining gamma/ delta T-cells, NK and other cells in the graft. So far, 32 pts have been treated in Tübingen and Rome. PBSC manipulation resulted in 4.5 (range 3.8-5) and 4.3 (3.7-5) log-depletion of alpha/ beta $T$ cells in Tübingen and Rome, respectively. The median number of CD34+ cells in the 2 centers was $12 \times 10^{6} / \mathrm{kg}(5-38)$ and $11 \times 10^{6} / \mathrm{kg}(8-40)$, respectively. Pts were given $107 \times 10^{6} / \mathrm{kg}$ (35-192) and $83 \times 10^{6} / \mathrm{kg}(34-242)$ CD56+ NK cells, respectively. The median number of gamma/delta T cells was $11.3 \times 10^{6} / \mathrm{kg}(5-$ 30 ) and $7.5 \times 10^{6} / \mathrm{kg}(1.4-25)$, respectively. No further post-transplant GvHD prophylaxis was given. The 15 Tübingen pts had advanced/refractory leukemias (8 ALL; 7 AML/MDS/JMML; 9 active disease, 6 in CR2-6). For these poor-prognosis pts, a RIC regimen (L-PAM, Thiotepa, fludarabine or clofarabine and OKT-3 or ATG) was used. All pts engrafted, the median time to PMN and PLT recovery being 10 (8-12) and 11 days (6-28) respectively. Three and 1 pts had grade II and III acute GvHD, respectively. Three pts experienced chronic GvHD (1 extensive). Three pts relapsed after HSCT and died; 1 pt died of multi-organ failure. 8 pts are in remission (median FU: 7 months, 5-15). The Rome cohort comprised 17 pts with ALL (13), AML (3) and NHL (1). All children but 1 had relapsed/refractory disease. In particular, 10 pts were transplanted in CR2 and 6 with more advanced disease. Myeloablative conditioning included fractionated TBI, Thiotepa, fludarabine/L-PAM and ATG. All pts but 1 engrafted, the median time to PMN and PLT recovery being $12(10-18)$ and 13 (8-15) days, respectively. Only 1 pt had grade I acute GvHD. No pt of the 12 at risk experienced chronic GvHD. At a median FU of 7 months (1-13), 14 pts are alive and disease-free; 3 pts relapsed (1 died) and 1 had fatal lung aspergillosis. In both cohorts, gamma/ delta T cells started to expand faster than alpha/beta T cells in the early post-HSCT period, whereas at day +100 alpha/beta T cells were predominant. These data indicate that transplantation of TCR alpha/beta/CD19 depleted cells from a haploidentical donor results in sustained engraftment, rapid immune recovery and low incidence of GVHD. The anti-leukemic efficacy of this approach needs to be evaluated with a longer follow-up.

\section{3}

Rapid and sustained engraftment of a single allogeneic ex-vivo expanded cord blood unit after reduced-intensity conditioning in adults. Preliminary results of a prospective trial

N. Milpied (1), B. Dazey (2), Z. Ivanovic (2), P. Duchez (2), S. Vigouroux (1), R. Tabrizi (1), A. Pigneux (1), M. Dilhuydy (1), K. Bouabdallah (1), T. Leguay (1), X. Lafarge (2), M. Sauvezie (1), J. Boiron (2)

(1)University Hospital (Pessac, FR); (2)EFSAL (Bordeaux, FR)

Engraftment rate and speed of a single $\mathrm{CBU}$ in adults remains unsatisfactory. Tx of $2 \mathrm{CBU}$ may overcome this problem with an 
increased rate of GVHd. Until now, attempts at using ex-vivo expanded $\mathrm{CBU}$ have been unsuccessful to promote long term engraftment. We report the results achieved in the first 8 Pts included in a PCT of transplantation of a single ex-vivo expanded allogeneic CBU. Eudract 2008-006665-81, Clinicaltrials. gov NCT 01034449.

Methods: Adults pts with an indication for SCT and unable to tolerate MAC (age $>45$, co-morbidities, previous HD therapy) were included if no Id sibling, no MUD 9 to 10/10 HLA matches and no CBU fulfilling the HLA matching $(\geq 4 / 6)$ and richness $\geq 3$ to $4 \times 10^{7} \mathrm{Nuc} \mathrm{C} / \mathrm{kg}$ before thawing) criteria. RIC consisted of Flu (40 mg/m²: $\mathrm{d} \times 5 \mathrm{~d}$ ), Cyclophosphamide (50 mg/kg x 1d) ICT 2 Gy. GVHd prevention consisted of MMF (d-3 to d28) and CSA from d-3. Graft engineering: $1 \mathrm{CBU}$ with $>2$ and $<3 \mathrm{TNC} / \mathrm{kg}$ and 4 to 6 HLA compatibilities was thawed, CD34+ cells were selected through magnetic device (Miltenyi) and ex-vivo expanded in SF medium ( HPO1-Mako) supplemented with SCF, FIt3I, GCSF and TPO during 12 days (Ivanovic, Cell Transplant 2011) CD34- cells were cryopreserved. On d0, expanded cells were washed and resuspended in HSA $4 \%$ and injected to the pt. Cd34- cells were thawed and injected $3 \mathrm{~h}$ later.

Results: From 03/2010 to 06./2011 8 pts have been included, med age 55y.o. (26-64) with AL: 3, Hodgkin's: 2, MDS: 3. Pts had received 1 to 3 lines of $T x$ (med :2). In 1 case the expanded product was contaminated. The pt then received a back-up $\mathrm{CBU}$, engrafted correctly and is AW at $19 \mathrm{~m}$. For the 7 other cases, the med fold expansion of CD34+ cells and TNC was 39 (29-75) and 390 (127-526) respectively, leading to a graft containing 1.3 to $13 \times 10^{6} \mathrm{CD} 34+$ cells $/ \mathrm{kg}$ (med: 2$)$. The CD34counterpart contained $3 \times 10^{6} \mathrm{CD} 3+/ \mathrm{kg}(1-5)$ and $0,9 \times 10^{6} \mathrm{CD} 19+$ cells $/ \mathrm{kg}(0,3-1,5)$. At d42, 6/7 pts who received the expanded graft had engrafted with $\geq 99 \%$ donors cells. The time to reach 500,1000 PMN's and 20000 plts was 7d (6-19), 8d (6-21) and 24d (0-39) respectively. 5 pts experienced AGVHd (1 grade IV). With a med FU of $15 \mathrm{~m}(6-21 \mathrm{~m}) 6 \mathrm{pts}$ are alive. For the 6 pts who engrafted with expanded product the chimerism remains full donor up to 1,5 year after Tx.

Conclusion: Ex-vivo expansion of $\mathrm{CBU}$ is feasible and reproducible, produces rapid, complete and sustained engraftment in adults after RIC.

\section{4}

Grade III-IV acute GvHD and treatment-related mortality are reduced among recipients of larger numbers of donor naïve CD8+ T-cells and plasmacytoid dendritic cell precursors in allogeneic BM grafts from unrelated donors: results from BMT CTN 0201

E. Waller (1), W. Harris (1), S. Devine (2), D. Porter (3), J. Ferrara (4), J. McCarty (5), C. Gonzalez (6), T. Spitzer (7), O. Krijanovski (8), A. Howard (9), C. Gurgol (10), B. Logan (11), D. Confer (12), C. Anasetti (13)

(1)Winship Cancer Institute (Atlanta, US); (2)Ohio State University Medical Center (Columbus, US); (3)University of Pennsylvania (Philadelphia, US); (4)University of Michigan (Ann Arbor, US); (5)Virginia Commonwealth University (Richmond, US); (6)Georgetown University (Washington DC, US); (7)Massachusetts General Hospital (Boston, US); (8)Sutter East Bay Medical Foundation (Berkeley, US); (9)National Marrow Donor Program (Minneapolis, US); (10)EMMES Corporation (Rockville, US); (11)Medical College of Wisconsin (Milwaukee, US); (12)National Marrow Donor Program (Minneapolis, US); (13)H. Lee Moffitt Cancer Center (Tampa, US)

Background: A subset of 161 patients from the 278 subjects randomized to receive BM grafts in the BMT CTN 0201 study had aliquots of the BM allografts analyzed at a central laboratory for the content of CD34+ progenitors and immune cell subsets by flow cytometry. Demographics, disease characteristics, conditioning regimens (all myeloablative) and GVHD immuneprophylaxis of the 161 patients were similar to the entire BMT CTN0201 population.
Methods: 46 progenitor and immune cell subsets were selected for study based upon the absence of a strong correlation with another graft subset (Pearson or Spearman correlation $>0.8$ ) and a priori interest. Graft characteristics were described separately for survivors and those who died and compared using a nonparametric Mann-Whitney Wilcoxon test. P-values were not adjusted for multiple comparisons, but only covariates for which the false discovery rate (q value) $<0.2$ are presented. Univariate and multivariable analysis (adjusting for factors known to be associated with transplant outcomes) were used to estimate the association of graft characteristics with transplant outcomes.

Results: Numbers of nucleated cells/kg, CD34+ cells/kg, CD8+ $\mathrm{T}$-cells $/ \mathrm{kg}$, and CD4+ T-cells/kg were not significantly associated with OS. Two cell subsets in the BM graft were significantly associated with transplant outcomes with univariate $p$-values $<0.01$ and q values of $<0.2$ : pre-plasmacytoid dendritic cells (pre-pDC) (median number $0.3 \times 10 \mathrm{E} 6 / \mathrm{kg} ; \mathrm{p}=0.007$ ), and naïve CD8+ T-cells (CD8N; median number of $1.3 \times 10 \mathrm{E} 6 / \mathrm{kg}$; $p=0.009$ ). Multivariable analysis including the content of either pre-pDC or CD8N showed better overall survival and decreased transplant-related mortality among patients receiving larger numbers of each donor cell subset. Grade $3 / 4$ acute GvHD was lower among recipients of more CD8N. Estimated 3 year OS for patients receiving $>0.3 \times 10 \mathrm{E} 6 \mathrm{pre}-\mathrm{pDC} / \mathrm{kg}$ was $55 \%$ versus $35 \%$ for recipients of $<0.3 \times 10 \mathrm{E} 6 \mathrm{pre}-\mathrm{pDC} / \mathrm{kg}(\mathrm{p}=0.025)$ and with $58 \%$ estimated 3 year OS for patients receiving $>1.3 \times 10 \mathrm{E} 6$ CD8N $/ \mathrm{kg}$ versus $35 \%$ for recipients of $<1.3 \times 10 \mathrm{E} 6 \mathrm{CD} 8 \mathrm{~N} / \mathrm{kg}$ $(p=0.01)$. Recipients of more than the median number of CD8N w significantly less grade III-IV acute GvHD 0.34 (0.12-0.96), $p=0.041$. Relapse rates were similar among recipients of larger versus lower numbers of donor pre-pDC and CD8N.

Conclusions: Donor pre-DC and naïve T-cells have important effects on clinical outcomes after allogeneic BMT from unrelated donors.

\section{5}

First-line plerixafor for stem cell harvesting; initial data from the Liverpool PHANTASTIC trial

R.E. Clark, J. Bell, S. Francis, G. Brearton, E. Jones, B. McEvoy, T. Callaghan, N. McGinnity, R. Salim

Royal Liverpool University Hospital (Liverpool, UK)

The standard of care for mobilising blood stem cells for autografting is chemotherapy (often cyclophosphamide) with G-CSF. Plerixafor is licensed in myeloma and lymphoma where chemotherapy + G-CSF have failed, but there are almost no data on the use of plerixafor as a first line mobilising agent. Here we present an interim report on the first 60 harvested patients that entered an ongoing trial of first line plerixafor with G-CSF in myeloma or lymphoma patients requiring an autograft (acronym PHANTASTIC).

All entrants were aged $\geq 18$ years and had no prior attempt at harvesting. G-CSF at $5 \mathrm{ug} / \mathrm{kg}$ was given on days $1-4$, and plerixafor at 2200 hours on day 4 , at a dose of $240 \mathrm{ug} / \mathrm{kg}$ if creatinine clearance $\geq 50 \mathrm{mls} / \mathrm{min}$ (59 cases) or $160 \mathrm{ug} / \mathrm{kg}$ if creatinine clearance $30-50 \mathrm{mls} / \mathrm{min}$ (1 case). Stem cell harvesting was carried out on day 5 and if necessary on days 6,7 and 8 with continuation of G-CSF and plerixafor, until the target yield of $4 \mathrm{x}$ $10^{6} \mathrm{CD} 34+$ cells $/ \mathrm{kg}$ recipient weight was achieved.

The median age was 54 years (range 20-68), and 26 patients had underlying myeloma, $26 \mathrm{NHL}$ and 8 Hodgkins disease. In the 30 days following G-CSF commencement, no SAE were seen though 5 patients subsequently died of disease progression before receiving their transplant. $118 \mathrm{AE}$ were seen, including 10 patients with mild transient gastrointestinal symptoms, insomnia and headaches which may have been plerixafor-related. The rest were attributable to known short term effects of leukapheresis (especially citrate toxicity).

48 of the 60 patients achieved the primary composite endpoint of BOTH an adequate stem cell harvest $\left(\geq 4 \times 10^{6} \mathrm{CD} 34+\right.$ cells/ $\mathrm{kg}$ in $\leq 2$ aphereses) AND no evidence of a neutrophil count 


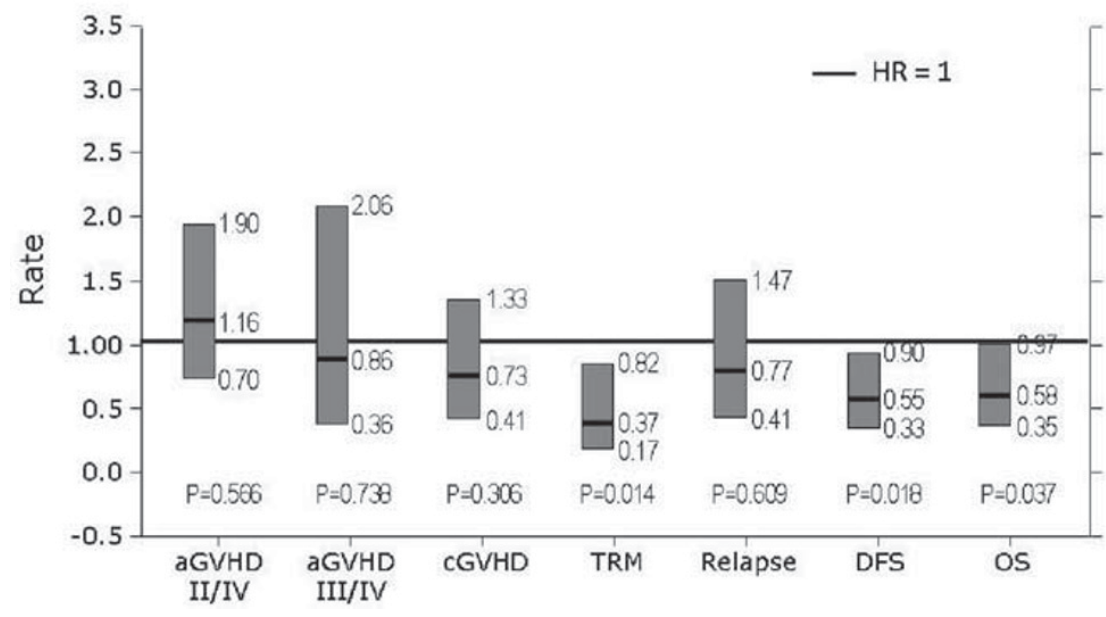

\section{B. Multivariate Results by Naïve CD $8+$ T-cells} (>1.3 $\times 10^{6} / \mathrm{kg}$ vs. $<1.3 \times 10^{6} / \mathrm{kg}$ )

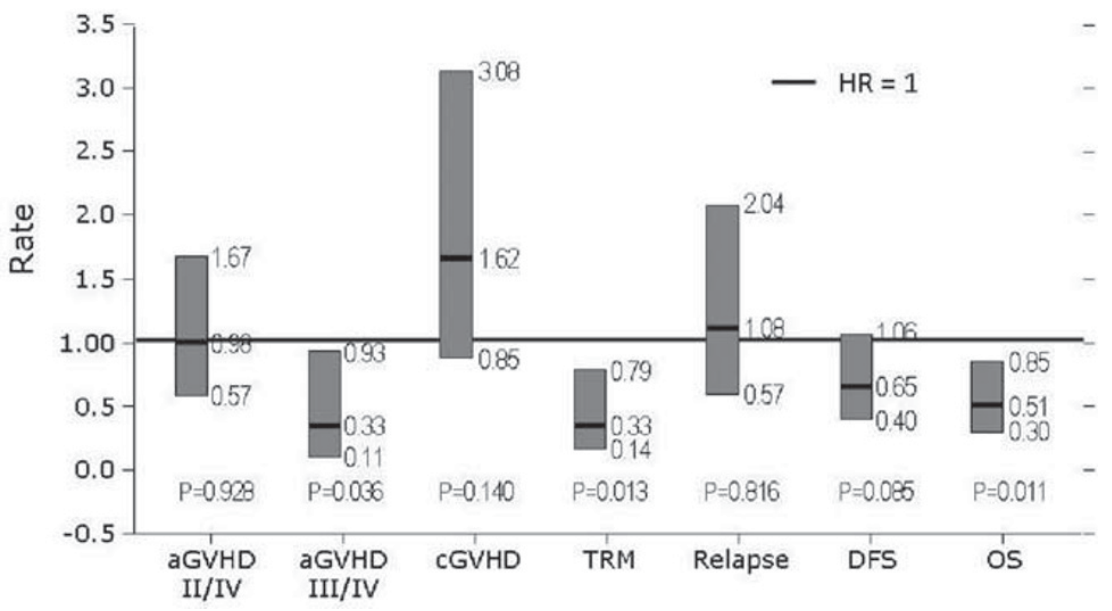

$<1 \times 10^{9} / \mathrm{L}$ over the 3 weeks after starting mobilisation. The median number of CD34+ cells collected was $5.49 \times 10^{6} / \mathrm{kg}$ (range 1.3521.11), and this was completed in 1 apheresis in 26 patients, in 2 for 25 patients, in 3 for 5 patients and in 4 for 4 patients.

46 of 60 successfully harvested patients are currently assessable for engraftment. The median time to neutrophil engraftment of $0.5 \times 10^{\%} / \mathrm{L}$ was 12 days, and for platelet engraftment to $50 \times 10 \% / L$ was 21 days. Of 21 cases assessable at 12 months post transplant, none have died though 4 have relapsed; the remaining 17 are alive and well.

We conclude that plerixafor is safe and effective as a first line mobilisation agent and the resultant stem cells can safely support a clinically effective subsequent autograft.

\section{6}

Prediction of CD34+ cell yield in haematopoietic cell product in children by peripheral blood CD34+ cell count P. Rujkijyanont, J. Hipps, K. Gan, J. Yang, C. Wang, T. Geiger, P. Eldridge, W. Leung

St Jude Children's Research Hospital (Memphis, US)

Background: Peripheral blood stem cells (PBSCs) are increasingly used as an alternative to bone marrow in autologous transplantations. In adult patients, the peripheral blood CD34+ cell count is a good predictor of CD34+ cell yield in apheresis. However, the determinants of stem cell yield in the pediatric population have not been well established.

Methods: We retrospectively studied 396 apheresis procedures in 301 pediatric patients. Receiver operating characteristic (ROC) curves based on pre-apheresis peripheral blood CD34+ cell count were generated to facilitate prediction of the optimal timing of PBSC collection. The associations between CD34+ cell yield and age and mobilization regimen were analyzed.

Results: Significant differences in CD34+ cell yield among different age groups were observed. Furthermore, higher CD34+ cell yields were obtained in patients receiving chemotherapy as a part of the mobilization regimen than those without chemotherapy. A correlation was noted between the CD34+ cell yield and blood surrogate markers, including white blood cell count, absolute neutrophil count, and pre-apheresis peripheral blood CD34+ cell count. A cutoff value of $>35$ CD34+ cells $/$ microliter in patients $<15$ years old and $>45$ CD $34+$ cells/microliter in patients $\geq 15$ years old were strong predictors of an adequate PBSC collection in one apheresis session. For clinical use, ROC curves and tables were generated to assist advance planning for PBSC collection. 
Conclusions: The pre-apheresis peripheral blood CD34+ cell count is most useful in predicting PBSC yield. Our new cutoff values have better operating characteristics in children than the conventional value of $20 \mathrm{CD} 34+$ cells $/ \mu \mathrm{L}$ used in adults.

\section{7}

Peripheral blood stem cell mobilisation in lymphoma patients candidates to autologous transplantation. Development of a predictive score

G. Rossi (1), C. Skert (1), E. Morello (1), C. Almici (1), L. Arcaini (2), C. Basilico (3), B. Botto (4), A. Castelli (5), G. Pica (6), F. Ripamonti (2), F. Salvi (7), A.M. Carella (6), G. Gaidano (5), A. Levis (7), A. Nosari (3), D. Russo (1), U. Vitolo (4) (1) Spedali Civili di Brescia (Brescia, IT); (2)University of Pavia (Pavia, IT); (3)Niguarda Hospital (Milan, IT); (4)AOU S. G. Battista (Turin, IT); (5)A.Avogadro University (Novara, IT); (6)AOU S. Martino (Genoa, IT); (7)Ospedale Civile (Alessandria, IT)

Autologous stem cell transplantation (ASCT) is potentially curative treatment of lymphoma. However peripheral blood stem cell (PBSC) mobilization may fail in some patients. New agents were recently developed which may improve the yield of PBSC. To optimize their use within current mobilization protocols we conducted a retrospective analysis on a large series of lymphoma candidates to ASCT, to identify factors influencing SC mobilization outcome, including potential "ongoing" markers, able to predict the risk of failure during mobilization attempts. Demographic and clinicopathological features at mobilization, as well as the number of circulating CD34+ cells and CD34+/WBC ratio calculated on the first day scheduled for collection, were analysed in 415 consecutive mobilization procedures in 388

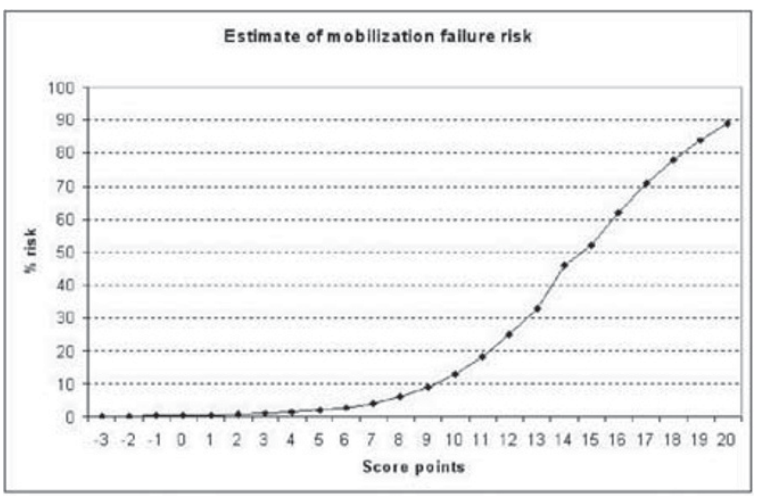

\begin{tabular}{|c|c|c|c|}
\hline Risk factors & Categories & Regression coefficient & Points \\
\hline $\cos 4 / \mu f$ & & $-0,02$ & \\
\hline \multirow[t]{9}{*}{ at CD34start } & $? 142$ & & -1 \\
\hline & $122-141$ & & 0 \\
\hline & $102-121$ & & 1 \\
\hline & $82-101$ & & 2 \\
\hline & $62-81$ & & 3 \\
\hline & $42-61$ & & 4 \\
\hline & $22-41$ & & 5 \\
\hline & $2-21$ & & 6 \\
\hline & $<2$ & & 7 \\
\hline$C D 34 / W b C(x 100)$ & & $.3,2$ & \\
\hline \multirow[t]{8}{*}{ at CD34start } & $>1,45$ & & -2 \\
\hline & $1,26-1,45$ & & 0 \\
\hline & $1,06-1,25$ & & 2 \\
\hline & $0,86-1,05$ & & 3 \\
\hline & $0,86-0,85$ & & 5 \\
\hline & $0,46-0,65$ & & 8 \\
\hline & $0,26-0,45$ & & 8 \\
\hline & $? 0,25$ & & 10 \\
\hline Cyclophosphamide & & 1,2 & \\
\hline & No & & 0 \\
\hline & Yes & & 3 \\
\hline
\end{tabular}

patients with lymphoma. Chemotherapy + G-CSF was used in $99 \%$ of mobilization procedures. Mobilization failed $\left(<2 \times 10^{6}\right.$ CD34+ cells $/ \mathrm{kg}$ collected) in $14,2 \%$ of attempts. At multivariable analysis, the use of cyclophosphamide as mobilizing chemotherapy, the number of circulating CD34+ cells and the CD34+/WBC ratio independently predicted mobilization failure. Using these three parameters a $<1 \%$ to $89 \%$ risk of mobilization failure can be predicted on the first day scheduled for collection, allowing to rationally add novel agents during mobilization attempts with chemotherapy and G-CSF at risk of failure.

\section{8}

Towards functional flow cytometry: the ability of Annexin $V$ assessment of CD34+ cells to predict colony-forming activity

R.C. Duggleby (1), L.J. Fry (2), S. Querol (3), R.C. Davy (2), D.A. Gibson (2), R.B.V. Horton (2), S.N. Mahmood (2), S.G. Gomez (2), J.A. Madrigal (1)

(1)Anthony Nolan Research Institute (London, UK); (2)Anthony Nolan Cell Therapy Centre (Nottingham, UK); (3)Banc de Sang i Teixits (Barcelona, ES)

Objectives: Non-viable CD34+ cells are commonly assessed by standard flow cytometry using the nuclear stain 7-Aminoactynomicin $\mathrm{D}$ (7AAD). 7AAD only detects necrotic/late apoptotic cells not earlier apoptosis, which engraft poorly in animal models of cord blood (CB) transplantation. The objective was to assess the ability of Annexin $\mathrm{V}$ staining (AnnV) to allow for early apoptosis and thus better predict stem cell function. This would then provide a rapid and robust method of predicting graft potency in thawed CB units.

Methods: AnnV labeling, in conjunction with 7AAD, was used to detect apoptotic events in CD34+ cells in fresh and thawed CB units by flow cytometry. Similarly, the effect of the presence of the cryo-protectant Dimethyl sulphoxide (DMSO) was observed. A parallel experiment was run, concurrently, whereby CD34+ cell numbers were quantified by standard ISHAGE based cell enumeration, and stem cell function assessed by colony forming unit (CFU) assay. To confirm the relationship, $\mathrm{CB}$ units and $\mathrm{CB}$ mononuclear cells were thawed and regularly reassessed for viability and function.

Results: Significant loss of CFU dose was observed following thawing of cryopreserved units ( $50 \%$ loss, $n=10)$ with little difference found in the viability by $7 A A D(<4 \%)$. However, significant numbers of $C D 34+a n n V+$ events were found within the 7AADgated population $(60 \%$ increase $A n n V+)$. The AnnV- dose correlated with the observed CFU in 78 fresh and 28 thawed samples $(r 2=0.53)$. In fresh samples $(n=8)$, low dose DMSO $(5-10 \%)$ was tolerated but high concentrations of DMSO (20$40 \%$ ) led to a rapid loss of CFU dose (40\% DMSO; $100 \%$ loss in 1 hour). This was only reflected by viability by AnnV ( $40 \%$ DMSO; $100 \%$ AnnV+, 40\% 7AAD+). Finally, using the relationship between CFU and viable cell dose, it was observed that the AnnV- dose predicted the observed CFU better than using 7AAD alone.

Conclusion: These data indicate that measuring the level of apoptosis is a better predictor of functional dose than the standard method of enumeration following strong cellular insult such as cryopreservation. In the case of DMSO the adverse effects are confined to apoptosis and consequently missed by the standard assessment. This flow cytometric technique, therefore, has the potential to be a rapid test of potency of $C B$ units that should be simple enough to allow standardization in a multi-centre scenario, and helps define best practice for the storage and use of $\mathrm{CB}$ units in transplantation. 
Infectious Complications - Viral

\section{9}

\section{Respiratory viral infections after HSCT}

R. Fernández, S. Melón, C. Fernández, P. Palomo, A.J. González, E. Alonso, S. González, A.P. González, C. Balado, M. Oña, C. Rayón, C. Vallejo

Hospital Universitario Central de Asturias (Oviedo, ES)

Introduction: Since respiratory viral infections (RVIs) are a significant cause of morbi-mortality in HSCT recipients, an active surveillance is needed.

Patients and Methods: This study includes 193 pts who underwent HSCT between January 2008 and December 2010 in our center (65 alloHSCT, 128 autoHSCT). All pts who developed respiratory symptoms were screened for $\mathrm{R} V s$. The respiratory samples (nasopharyngeal wash, throat swabs, or BAL) were tested by immunofluorescence and by molecular methods for human: influenza A and B viruses (IV-A, IV-B), respiratory syncytial virus (RSV), parainfluenza viruses (PIVs 1 and 3 ), adenovirus (AdV), metapneumovirus (MPV), rhinovirus (RV), coronavirus (CoV), and bocavirus (BoV).

Results: During the study period, 65 alloHSCT (100\%) and 57 autoHSCT $(44,5 \%)$ were searched at least once, and 672 samples were processed for RV detection. The median number of samples/pt was 3,46 (range: 0-23; alloHSCT: 7,76, and autoHSCT: 1,32). A total of $191 \mathrm{RVI}$ episodes (147 in alloHSCT, 43 in autoHSCT) were diagnosed in 85 pts. The median number of RVI episodes/pt was 2,24 (range: 1-10). The viral distribution was: 86 RV (45\%), 39 RSV (20,5\%), 36 PIV (19\%), 30 BoV $(15,7 \%), 28$ IV $(14,7 \%), 6$ MPV $(3,1 \%), 1$ CoV $(0,5 \%)$, and $1 \mathrm{AdV}(0,5 \%)$. The types of IV found were: 17 pandemic 2009 H1N1, 3 seasonal IV-A, and 7 IV-B. There were 35 mixed $\mathrm{RVI}$ episodes. The median presentation was at day +74 postHSCT. $88 \%$ episodes were classified as URTI, and $12 \%$ as LRTI $(7,3 \%$ bronchitis and $4,7 \%$ pneumonia; the majority of them in allo-HSCT). The RVs responsible for the LRTIs were: RSV (11), H1N1 (5), RV (3), IV-B (2), PIV (1), and MPV (1). Risk factors for progression to LRTI were: 1) CMV seropositivity $(83 \%$ vs $16 \%, p=0,037) ; 2)$ lower IgG $(0,46$ vs $0,61 \mathrm{~g} / \mathrm{L}$, $\mathrm{p}=0,019) ; 3$ ) Iymphopenia (295 vs $\left.\left.855 / \mathrm{mm}^{3}, \mathrm{p}=0,035\right), 4\right)$ use of corticosteroids ( $56 \%$ vs $14 \%, p=0,01)$, and 5 ) use of $>1$ immunosuppressant $(26,7 \%$ vs $5,3 \%, p=0,047)$. The overall mortality and the attributed mortality within 1 month from RVI diagnosis was $5,7 \%$ and $3,1 \%$ ( 6 cases: 3 RSV, and $3 \mathrm{H} 1 \mathrm{~N} 1$ ).

Conclusions: 1) RVI episodes are frequently discovered, particularly among the alloHSCT recipients, when an intense screening activity is implemented; 2 ) In our series, RV and BoV were the most common, and rarely associated with LRTI; 3) RSV and IV (specially H1N1) can lead to serious disease with high mortality; 4) lymphopenia, low IgG, corticosteroids, and CMV seropositivity seem to be associated with progression to LRTI.

TABLE 1: Screening activity for RV detection and frequency of RVI episodes

\begin{tabular}{|c|c|c|}
\hline & $\begin{array}{l}\text { AlloHSC T } \\
(\mathrm{n}=65)\end{array}$ & $\begin{array}{l}\text { Auto HSCT } \\
(\mathrm{n}=128)\end{array}$ \\
\hline Patien's sampled (\%) & $65(100 \%)$ & $57(44,5 \%)$ \\
\hline Total No of samples & 502 & 170 \\
\hline Medinm number of sapaples per patient (range) & $7,76(1.23)$ & $1,32(0.11)$ \\
\hline No of patients with a docunented R VI (\%) & $54(83,1 \%)$ & $31(24,2 \%)$ \\
\hline Total No of RVI eqiso des & 148 & 43 \\
\hline Medinen number of RVI piso des per patien' (renge) & $2,26(0.10)$ & $0,34(0.4)$ \\
\hline Median tine to first RVI episo de (diys) & 126 & 16 \\
\hline No of UR TI episo des ( $\%$ of totalRVI episodes) & $130(88,4 \%)$ & $37(86 \%)$ \\
\hline $\begin{aligned} \text { No of LRTI episodes } \\
: \text { Brenchitis }(\%) \\
\text { - Pneunonia }(\%) \\
\end{aligned}$ & $\begin{array}{l}10(6,8 \%) \\
7(4,7 \%) \\
\end{array}$ & $\begin{array}{l}4(9,3 \%) \\
2(4,6 \%)\end{array}$ \\
\hline No of Influenza virus foise des ( $A$ and $B$ ) & 20 & 8 \\
\hline No of HPIV episodes & 25 & 11 \\
\hline No of HRSV episodes & 33 & 6 \\
\hline No of HRV V pisedes & 48 & 38 \\
\hline No of HBoV quiso des & 21 & 9 \\
\hline No of HMPVV episedes & 5 & 1 \\
\hline No of HCOV episodes & 1 &. \\
\hline No of HAdV epise des & 1 & . \\
\hline No of nixed RVI & 26 & 9 \\
\hline
\end{tabular}

TABLE 2: Clinical characteristics and outco me of most common symp to matic RVI

\begin{tabular}{|c|c|c|c|c|c|}
\hline & $\begin{array}{l}\text { Influenza } \\
\text { ( } \mathrm{A} \text { and } \mathrm{B} \text { ) }\end{array}$ & HRSV & HPIV & HRV & HMPV \\
\hline Typ e of HSCT (Allo-/Auto-) & 2018 & $33 / 6$ & $25 / 11$ & $48 / 38$ & $5 \pi$ \\
\hline $\begin{array}{l}\text { Month of p resentation (most } \\
\text { frequent) }\end{array}$ & $\begin{array}{l}\text { Jan }(8) \\
\text { Nov }(s) \\
\operatorname{Dec} .(s)\end{array}$ & $\begin{array}{l}\text { Jan.(15) } \\
\text { Feb.(8) } \\
\text { March(4) } \\
\text { Nov }(4) \\
\operatorname{Dec}(4)\end{array}$ & $\begin{array}{l}\text { May (7) } \\
\text { June (7) } \\
\text { April (6) } \\
\operatorname{Dec}(5)\end{array}$ & $\begin{array}{l}\text { Oct (13) } \\
\text { June(g) } \\
\text { May }(7) \\
\text { Sept(z) } \\
\text { Feb. }(\gamma)\end{array}$ & $\begin{array}{l}\text { May (2) } \\
\text { June (2) }\end{array}$ \\
\hline Period alter SCT (me dien dyys) & 293 & 175 & 217 & 245 & 244 \\
\hline Lymphocytes (median, /mm) & 665 & 840 & 450 & 610 & 1200 \\
\hline IgG (me dian, mg/dl) & 585 & 607 & 730 & 560 & 670 \\
\hline $\begin{array}{l}\text { Site of infection: } \\
\text { - URTI } \\
\text { - LRTI (Bronchitis) } \\
\text { - LRTI (Pneumonia) }\end{array}$ & $\begin{array}{l}21 \\
2 \\
5\end{array}$ & $\begin{array}{l}28 \\
? \\
4\end{array}$ & $\begin{array}{l}35 \\
1 \\
-\end{array}$ & $\begin{array}{l}62 \\
3 \\
.\end{array}$ & 6 \\
\hline Antiviral ther apy (tocel tre sted) & 22 & 5 & $\cdot$ & $\cdot$ & $\cdot$ \\
\hline $\begin{array}{l}\text { Outcome } \\
\text { - Resolution } \\
\text { - Overall mortality } 1 \mathrm{~m} . \\
\text { - Direct mortality }\end{array}$ & $\begin{array}{l}24 \\
1 \\
3\end{array}$ & $\begin{array}{l}35 \\
1 \\
3\end{array}$ & $\begin{array}{l}35 \\
1 \\
\cdot\end{array}$ & $\begin{array}{l}63 \\
3 \\
.\end{array}$ & 6 \\
\hline
\end{tabular}

0140

GPI-anchor negative T-cells with impaired EBV-specific

T-cell function after Alemtuzumab mediated T-cell depletion in the context of allogeneic haemopoiteic stem cell transplantation

E.M. Wagner, A. Kouka, C. Götze, M. Theobald, W. Herr, R.G. Meyer

Medical Center of the Johannes Gutenberg University (Mainz, DE)

Alemtuzumab is a monoclonal anti-CD52-antibody frequently used to deplete T cells (TC) in the context of allogeneic hemopoietic stem cell transplantation (HSCT). We recently showed long-term persistence of CD52-negative $T$ cells reconstituting in patients after Alemtuzumab mediated T-cell depletion (TCD). The lack of CD52 on the cell surface was due to missing glycosyl-phosphatidyl-inositol (GPI)-anchors. We found that the persisting $\mathrm{GPI}$-anchor negative T cells exhibit reduced CMV-specific T-cell function. Since EBV-reactivation and EBVassociated diseases (for example post transplantation lymphoproliferative disorder PTLD) are life-threatening complications after HSCT, we also investigated on EBV-specific T-cell functions of GPI-anchor negative T cells to estimate their impact on the clinical course of our patients with EBV-viremia $(n=6)$ and EBV-associated PTLD $(n=4)$.

PBMC were frozen at different time points in the course of EBV-reactivation and PTLD-therapy. We identified EBV-specific TC by EBV-peptide/HLA-A2 Tetramer staining and analyzed peptide-specific cytokine production by intracellular FACS and ELISPOT-assay. Antigen-specific proliferation was tested in CFSE-assays and cytotoxicity was determined in chromium release assays. EBV-peptide loaded dendritic cells of our patients, peptide loaded K562-cells, as well as autologous EBVblasts (LCL) were used as stimulators in the functional assays. GPI-anchor negative TC showed a reduced EBV-specific TC-response. Patients with EBV-PTLD had no EBV-specific TC prior to the occurrence of PTLD. In patients with progressive or persistent disease, EBV-specific TC were first detected after clinical recovery. Whenever EBV-reactive TC were found, they were mainly present in the $\mathrm{Gl}$-anchor positive TC-compartment.

In conclusion, we confirmed that GPI-anchor negative T cells reconstituting after Alemutzumab mediated TC-depleted HSCT show an impaired antiviral function. Our data support the hypothesis that these functionally impaired GPI-anchor negative TC, persisting years after HSCT, are partly responsible for some of the known viral complications in patients undergoing Alemtuzumab based conditioning regimen. We have demonstrated earlier that application of donor lymphocyte infusions (DLI) replenishes the GPI-anchor positive TC-compartment. Therefore, We hypothesize that DLI also improve immunologic 
control of virus associated diseases associated with CMV and EBV-reactivations.

\section{1 \\ Correlation between BK viraemia and clinical response to cidofovir treatment: results of an IDWP-EBMT retrospective study \\ S. Cesaro, M. Pillon, R. Martino, I. Panizzolo, F. Al-Mohareb, W. Schroyens, C. Nozzoli, P. Barba, M. Faraci, F. Fagioli, B. Cappelli, C. Cordonnier, Y. Fløisand, J. Greil, G. Tridello, S. Santarone, M. Aljurf on behalf of the Infectious Diseases Working Party}

Background: Therapy with cidofovir (CDV) is often used in patients with BK-virus-associated hemorrhagic cystitis (BKV-HC) after allogeneic hematopoietic stem cell transplantation (HSCT) but the modification of BKV load on blood during antiviral therapy has been scarcely investigated in previous studies. The aim of this study is to retrospectively assess the relationship between CDV therapy, clinical response and modification of BKV viremia.

Patients and Methods: Over 2 years, 32 cases of $\mathrm{HC}$ with documented BKV viremia and treated with i.v. CDV were collected among 13 centres.

Results: The patients had a median age of $24 \mathrm{y}$, range 3.4-62 (12 of them < 18 year-old at HSCT), mainly affected by acute leukemia (22 patients), and in hematological CR at HSCT (21 patients). Donor origin was a sibling in 10 patients $(31 \%)$ and an unrelated/mismatched family donor in 22 patients $(69 \%)$. The source of stem cells was BM, PB, or CB, in 13, 11 and 8 patients, respectively. Conditioning regimen was mainly based on busulfan (21 patients, $66 \%$ ) or on TBI (7 patients, $22 \%$ ). ATG was used as prophylaxis of GVHD in 17 patients $(53 \%)$. $\mathrm{HC}$ occurred at a median time of 29 days, range $6-28$, from HSCT and was scored as grade I, II, III, and IV in 4, 4, 18, and 6 patients, respectively. Concomitant viral infection was recorded in 12 patients for CMV, and in 3 patients for adenovirus and EBV, respectively. In 31 of 32 patients evaluable, therapy with CDV was associated with complete response in 19 patients $(60 \%)$, partial response in 8 patients $(25 \%)$ and no improvement in 4 patients. Renal toxicity was reported in 9 patients (grade I, 4, grade II, 3, grade III, 2) whereas 1 patient had both grade III renal and cardiac toxicity. 12 of 31 patients had BKV viremia assessment before every CDV administration. In 10 of 12 patients, a virological response was observed, as follows: reduction of $1 \log$ in 4 patients, reduction of $3 \log$ in 4 patients, and reduction $>3$ log in 2 patients. All these 10 patients showed a clear improvement of $\mathrm{HC}$ during CDV therapy: 9 had a complete response and $1 \mathrm{had}$ a partial response. In the 2 patients without virological response no clinical benefit was observed from CDV therapy with CDV. After a median follow-up of 259 days (range 62-581), the OS of the whole cohort was $66 \%$. Conclusion. 1-3 log-reduction of BKV viremia is associated with clinical recovery or improvement from HC. Prospective studies are needed to confirm this data.

\section{2}

Impact and risk factors of cytomegalovirus reactivation in allogeneic haematopoietic stem cell transplantation: a multicentre prospective study on behalf of GITMO (Italian Cooperative Group of HSCT)

A. Proia (1), A. Severino (1), C. Girmenia (2), A. Bacigalupo (3), A. Rambaldi (4), W. Arcese (5), S. Sica (6), A. Locasciulli (1) (1)Osp. S. Camillo (Rome, IT); (2)Policlinico Umberto I (Rome, IT); (3)Ospedale S. Martino (Genoa, IT); (4)Ospedali Riuniti Bergamo (Bergamo, IT); (5)Università Tor Vergata (Rome, IT); (6)Policlinico A. Gemelli (Rome, IT)

Between January 2009 and December 2010, 1060 patients (median age 44 years, range 1-72), 604 males and 456 females undergoing hematopoietic stem cell transplant (HSCT) were serially monitored for cytomegalovirus (CMV). 645 pts $(60,8 \%)$, had a myeloablative transplant (MAT); $415(39,1 \%)$ having a reduced-intensity conditioning (RIC). Matched sibling/ family was $573(54 \%)$ and unrelated donors was $482(45,4 \%)$. Graft source was peripheral blood stem cells (PBSC) in 717 $(67,6 \%)$, bone marrow $(\mathrm{BM})$ in $266(25 \%)$, and cord blood (CB) in $77(7,2 \%)$. Anti-thymocyte globulin was administered in 541 $(51 \%)$. Donor (D)/Recipient (R) CMV serostatus was analized as follows: $D-/ R-, D+/ R-, D+/ R+, D-/ R+$. Serial weekly monitoring for CMV reactivation was performed using either antigenemia or viremia according to Center policy. CMV reactivation was documented in 466 patients $(43,9 \%)$ at a median of 80 days post HSCT (range 1-365). CMV reactivation was observed with incidence of $56 \%, 46,7 \%, 24 \%, 15 \%$ rispectively in $\mathrm{D}-/ \mathrm{R}+, \mathrm{D}+/ \mathrm{R}+, \mathrm{D}+/ \mathrm{R}-$, and $\mathrm{D}-/ \mathrm{R}-$, according to pre-transplant CMV serostatus.

On univariate analysis, the significant risk-factors identified for CMV reactivation included $\mathrm{D}-/ \mathrm{R}+$ and $\mathrm{D}+/ \mathrm{R}+\mathrm{CMV}$ serostatus $(P<0.0001)$; matched unrelated versus sibling donor $(P<0.0001)$, and acute graft versus host disease grade 2-4 versus $0-1(P<0.0001)$. Hematological diagnosis and disease status at transplant, graft source (BM versus PBSC versus CB), and the type of conditioning regimen (MAT versus RIC) did not influence significantly the risk of CMV reactivation.

725 patients $(68,3 \%)$ are alive and well, at a median follow-up of 7 months (range 0,3-12).

CMV reactivation had no significant impact on overall survival. Regarding pretransplant CMV serostatus, survival was significantly lower in $\mathrm{D}-/ \mathrm{R}+$ patients compared with the other groups $(P<0.005)(74 \% \mathrm{D}-/ \mathrm{R}-;$; $65 \% \mathrm{D}+/ \mathrm{R}-; 66 \% \mathrm{D}+/ \mathrm{R}+; 54 \%$ $\mathrm{D}-(\mathrm{R}+)$. Furthermore survival was highly affected by timing of reactivation: $41 \%$ in patients reactivating before day +30 and $69 \%$ in those with delayed reactivation $(P<0.0001)$. These data, obtained in a large multicenter series, confirm prospectively the impact of pre-transplant serostatus on CMV reactivation. Interestingly, SC source has no impact in this cohort, while type of donor (familiar or not) is a significant factor. The timing of reactivation proved to influence survival significantly.

\section{3}

Combined prospective monitoring of human

cytomegalovirus load and virus-specific protective T-cell response in young patients receiving allogeneic haematopoietic stem cell transplantation

F. Locatelli (1), D. Lilleri (2), P. Zelini (2), A. Chiesa (2), V. Rognoni (2), G. Giorgiani (2), F. Bonetti (2), G. Palumbo (1), R.M. Pinto (1), F. Rossi (1), F. Cocca (1), D. Pagliara (1), M. Zecca (2), G. Gerna (2)

(1)IRCCS Bambino Gesu' Children's Hospital (Rome, IT); (2)Fondazione IRCCS Policlinico S. Matteo (Pavia, IT)

In patients receiving allogeneic HSCT, outcome of HCMV infection results from balance between viral load/replication and its control by the reconstituting T-cell response. Using a cut-off of 30.000 HCMV DNA copies/ml blood for initiation of pre-emptive therapy and cut-offs of 1 and 3 virus-specific CD4+ and CD8+ T-cells/uL blood for defining T-cell protection, we conducted a prospective study aimed at verifying whether patients attaining such immunological cut-offs were protected from HCMV reactivation. A total of 131 young patients (median age, 8 years; range, $1-23$ years, 42M/89F), receiving allogeneic HSCT from January 2007 through January 2010, were studied. Patients had either malignant $(n=98)$ or non-malignant disorders $(n=33)$. Antiviral treatment was stopped following two consecutive negative blood controls. The frequency of HCMV-specific CD4+ and CD8+ T-cells producing IFN-gamma and IL-2 was determined by flow cytometry, following in vitro stimulation with autologous monocyte-derived, HCMV-infected, dendritic cells. In the first 3 months after transplantation, 55/89 (62\%) HCMV-seropositive patients had infection and $36 / 55(65 \%)$ were treated preemptively, whereas only $7 / 42(17 \%)$ HCMV-seronegative patients developed HCMV infection and $3 / 7(43 \%)$ were treated. 
Overall, only 1 patient developed HCMV disease (gastritis), which resolved under anti-viral treatment. After 12 months, 76 $\mathrm{HCMV}$-seropositive and $9 \mathrm{HCMV}$-seronegative patients (cumulative incidence: $90 \%$ and $21 \%$, respectively, $\mathrm{p}<0.01$ ) displayed protective levels of HCMV-specific T cells. Overall, 80 of these $85(95 \%)$ patients showed spontaneous control of HCMV infection, not requiring any additional antiviral treatment. Five patients after reaching protective T-cell levels needed pre-emptive therapy, because they developed GvHD. IFN-gamma+/IL-2+ $\mathrm{CD} 4+$ and $\mathrm{CD} 8+\mathrm{T}$ cells were found to emerge later in patient PB than IFN-gamma+ T cells. In summary, HCMV infection was much more frequent in HCMV-seropositive patients than in seronegative patients. As a consequence, the virus-specific T-cell response was more frequent in HCMV-seropositive patients; however, protective activity of the T-cell response against HCMV was detected in both seropositive and seronegative patients. Protection was stable and long-lasting, unless steroid therapy for GvHD was administered, this leading in some cases to episodes of HCMV reactivation. Monitoring of HCMV infection may be tailored on reconstitution of specific immunity.

\section{4}

Persistent cytomegalovirus reactivation and cytomegalovirus UL97 mutations in the seropositive haematopoietic stem cell transplantation setting

H. Xiao, Y. Luo, S. Fu, J. Shi, Y. Tan, J. He, W. Xie, X. Lai, X. Ye, X. Yu, Z. Cai, M. Lin, H. Huang

Zhejiang University School of Medicine (Hangzhou, CN)

Objectives: The emergence of cytomegalovirus (CMV) drug resistance, favored by long-term exposure to antiviral drugs and by profound immunosuppression, is a growing therapeutic challenge in allogeneic hematopoietic stem cell transplantation (allo-HSCT). The majority of ganciclovir (GCV) resistance is associated with mutations in the CMV phosphotransferase (UL97) gene. The aim of our study was to evaluate the prevalence of persistent CMV reactivation and CMV UL97 mutations at a single center in China, endemic for asymptomatic CMV infection.

Methods: One hundred and thirty-eight pairs of patients and their unrelated donors (URDs) and 102 pairs of patients and their HLA-identical sibling donors, who underwent HSCT at our center from 2001 to 2009 were included in this study. Patients were monitored for detection of CMV infection for $\geq 2$ years. CMV UL97 gene amplifying by Nested-PCR and sequencing were performed if viral load persisted for $>21$ days on appropriate antiviral treatment.

Results: (1) All patients and almost all donors except one were CMV seropositive before HSCT. In the URD transplantation cohort, 44 patients $(31.88 \%)$ experienced CMV reactivation infection pre-HSCT, as did 28 patients $(27.45 \%)$ in the sibling HSCT cohort. (2) 134 (55.8\%) patients had experienced early CMV infection (within day +100 ) with a median onset of 27 days (range 2-64) after HSCT. 20.9\% developed infection during granulocytopenia and $79.1 \%$ did so after neutrophil recovery. The majority of patients developed CMV-positive antigenemia without disease, only $9(6.7 \%)$ patients developed CMV disease ( 7 patients with CMV pneumonia and 2 patients with CMV enteritis). Unrelated donors $(p=0.044, R R=1.440)$, patients experiencing CMV reactivation pre-HSCT $(p=0.012$, $R R=0.632$ ) were risk factors for early CMV infection. (3) Patients experiencing early CMV infection were with faster clearance of viremia after GCV treatment. $73(30.4 \%)$ patients developed $\geq 1$ episode of CMV viremia beyond day +100 (late infection) and persistent reactivation occurred in $30(41.1 \%)$ patients. However in those persistent infection cases, we had not detected the 7 most common GCV-resistance mutations at UL97 codons (M460V/I, H520Q, C592G, A594V, L595S, and C603W). Two novel mutations CMV UL97 H602Q and D605E were found, especially D605E mutation occurred in $16(53.3 \%)$ patients. Conclusion: CMV UL97 D605E and H602Q mutations may contribute to persistent CMV reactivation in allo-HSCT setting.

\section{5}

Adenovirus load in stool predicts invasive dissemination in paediatric recipients of haematopoietic stem cells L. Feghoul (1), J. Dalle (2), J. Lambert (1), M. Ouachée (2), C. Scieux (1), A. Baruchel (2), F. Simon (1), J. LeGoff (1) (1)Hôpital Saint-Louis (Paris, FR); (2)Hôpital Robert Debré (Paris, FR)

Introduction: Adenovirus infections are a major cause of morbidity and mortality in pediatric transplantation of hematopoietic stem cells (HSCT). The intestinal tract is the most common cause of systemic adenovirus infections in this population. Early detection and quantification of adenoviral infections in stool for predicting the risk of dissemination represent a major challenge

Objective: To define the threshold of adenoviral DNA viral load (VL) in stool associated with a risk of dissemination in blood. Methods: Between September 2010 and April 2011, all patients hospitalized in HSCT unit were weekly tested for adenovirus in blood and stool. Detection and quantification of adenoviruses were performed by real-time PCR with a limit of quantification of 200 copies/ml (Argene, Verniolle, France). The predictive value of dissemination in blood was determined by $V L$ in pairs of plasma and stool as close as possible (median $=2$ days). Results: Among the 51 patients 28 had a digestive infection, 16 of the 28 patients presented positive detection in blood and 9 with a plasma VL > 10000 copies $/ \mathrm{ml}$. The predictive value was calculated from 72 pairs plasma/stool from 26 patients. VL stool was significantly higher in viremic patients $\left(8.62 \times 10^{8} \mathrm{copies} / \mathrm{ml}\right)$ than non-viremic patients $\left(2.74 \times 10^{8}\right.$ copies $\left./ \mathrm{ml}\right)\left(p<10^{-5}\right)$. The threshold of 100000 copies $/ \mathrm{ml}$ in stool is associated with a risk of dissemination in blood with sensitivity and specificity were respectively 96\% [C|95\%:78\%-100\%] and 65\% [50\%-78\%]. The positive and negative predictive values were respectively $56 \%$ [40\%-72\%] and 97\% [84\%-100\%].

Conclusion: In a pediatric population of HSC transplants, the detection of adenovirus in blood is preceded by a digestive replication. Quantification in stool is predictive of risk of dissemination in blood. These results must be considered for the monitoring of adenovirus infections and pre-emptive treatment in patients at risk of disseminated infections.

\section{Early Complications}

\section{6}

Determining timing of late engraftment and graft failure following single cord, unrelated transplantation: An Analysis of the Eurocord Registry

R. Saccardi (1), A. Ruggeri (1), M. Labopin (2), M. P. Sormani (3), M. Eapen (1), C. Kenzey (1), W. Chaves (1), R. Cunha (1), E. Gluckman (1), V. Rocha (1)

(1)Eurocord Registry (Paris, FR); (2)EBMT Office (Paris, FR); (3)University of Genoa (Genoa, IT)

Introduction: An overall incidence of $10-20 \%$ of graft failure (GF) is reported after cord blood transplantation (CBT). The decision and timing to initiate search for an alternative graft and proceed to the 2 nd transplant is particularly intriguing. After transplantation, the probability of engraftment increases progressively to a maximum and then gradually decreases. We analysed engraftment kinetics after CBT to develop an evidence-based strategy that would facilitate the decision process for a 2nd transplant. Patients and Methods: We investigated engraftment kinetics in a population of 1268 patients who received single, unrelated CBT for acute leukaemia (AL) in complete remission (CR) after myeloablative conditioning regimen. All patients were transplanted at EBMT Centers and reported to the Eurocord Registry from 1994 to 2010 . Ratio of lymphoid/myeloid leukaemias was $813 / 455$, reflecting a major proportion of paediatric 
patients over adults (929/338). Patients were transplanted in first $(47 \%)$, second $(45 \%)$, or third or subsequent remission $(8 \%)$, respectively. Median (range) age at transplant was $8.7(0.3-63)$ years. Median TNC count at freezing was $5.1(1.1-41.83) \times 10^{7}$ ) $\mathrm{Kg}$. The probability density model was used, that described the probability to engraft at time points after CBT and considered competing events, such as early deaths.

Results: The cumulative incidence of engraftment was $86 \%$ at 60 days with a median engraftment time of 24 days. The probability to engraft peaked at day 21 and gradually decreased thereafter to day 65 . The residual probability to engraft was $21.5 \%$ on day 31 (half of the median) and dropped to $5 \%$ on day 42 . NRM was $26 \%, 29 \%, 37 \%$ for patients engrafting before day32 , from day-32-42 and over day-42, respectively. Graft failure was reported in 166 patients and data on treatment were available for 118. Forty-one died untreated at a median of 51 days, 10 experienced an autologous recovery and 77 underwent a second transplant (34\% autologous rescue). with an overall survival of $30 \%$ at 2 years.

Conclusions: The probability of engraftment after single CBT for AL in CR peaks at day 21 and decreases rapidly after day 31. Rescue actions, such as the search of another graft, should be considered beyond this time and a 2 nd transplant should be carried out soon after day 42 . This model can be applied to any type of transplantation and is particularly useful in the setting of high risk of graft failure, such as CBT.

\section{7}

Impact of antithymocyte globulin-containing conditioning regimens on patients undergoing allogeneic stem cell transplantation for progressive myelodysplastic syndrome: a report from the SFGM-TC Group I. Yakoub-agha, G. Damaj, M. Robin, S. Vigouroux, A. Garnier, M. Michallet, J. El-Cheikh, B. Lioure, J. Bay, P. Bordigoni, N. Contentin, N. Maillard, E. Deconinck, G. Guillerm, F. Legrand, E. Marry, M. Mohty on behalf of the SFGM-TC

Due to a risk of relapse of underlying disease in pts transplanted with progressive malignancy, the use of ATG, incorporated within the conditioning prior to allo-SCT, is still controversial. We report here on a study of 245 consecutive patients transplanted between Jan 1999 and Dec 2009 for progressive MDS.

Patients: Inclusion criteria included patients aged over 18 who received allo-SCT from either a sibling $(n=153)$ or HLA- matched unrelated donor $(10 / 10)(n=86)$ for progressive MDS or AML/RAEB-t.

Results: 239 pts were analyzed, including 154 males. According to the WHO classification at diagnosis, 85 pts had RA/RARS/ RCMD, 86 RAEB1, 62 REAB2 and 6 RAEB-t/AML. Sixty-six pts had progressed to a more advanced disease before allo-SCT. At diagnosis, 102 pts had an IPSS int-2 or higher. Cytogenetic was recorded as favorable $(n=109)$, inter $(n=61)$, unfavorable $(n=63)$ and missing $(n=6)$. Disease status at transplant was established as follows: relapsed or refractory disease $(n=106)$ and untreated or stable disease without hematological improvement $(n=133)$. Median age at transplantation was 53 years (20-70). Pts received myeloablative conditioning $(n=105)$ and nonmyeloablative $(n=134)$ including busulfan-based regimens $(n=127)$, TBI-based regimens $(n=92)$ or other alkylating-agentbased regimens $(n=20)$. In this series, 95 patients $(40 \%)$ received ATG as part of conditioning ('ATG' group), whereas 144 did not ('no-ATG' group). As of April 1st 2011, median followup in survivors was 50 months (IQR, 33-92) with 59 pts died of relapse and 77 of TRM. The estimated 3-yr OS and EFS was respectively $42 \%$, and $32 \%$. The probability of relapse, OS and EFS at 3 years was not significantly different between the two groups. In contrast, the cumulative incidence of grade 2-4 aGVHD was $48 \%$ in the no-ATG group and $30 \%$ ATG group $(\mathrm{P}<.001)$ and the cumulative incidence of grade $3-4$ aGVHD was $24 \%$ and $11 \%$ respectively $(P<.001)$. Although the cumu- lative incidence of chronic GVHD was similar in the no-ATG and ATG groups $(64 \%$ vs $46 \%, p=.15)$, a trend for a lower TRM was observed in the ATG group (22\% vs 31\%, $\mathrm{p}=.06)$. In multivariate analysis, the absence of ATG was the strongest parameter associated with an increased risk of acute grade 2-4 $[\mathrm{HR}=2.28,95 \% \mathrm{Cl}: 1.39-3.74, \mathrm{p}=.001]$ and grade $3-4 \mathrm{GVHD}$ $[\mathrm{HR}=2.19,95 \% \mathrm{Cl}: 1.04-4.61, p=.035]$.

Conclusion: The addition of ATG to the conditioning, resulted in a decreased incidence of aGVHD without increasing relapse rates and compromising survival.

\section{8}

Validation of the classical EBMT score and the modified EBMT score in patients undergoing allogeneic haematopoietic transplantation with reduced-intensity conditioning

P. Barba (1), D. Valcarcel (1), F. Fernandez-Avilés (2), R. Martino (3), M. Rovira (2), J.L Piñana (3), S. Brunet (3), E. Carreras (2), J. Sierra (3)

(1)Hospital Universitari Vall d'Hebron (Barcelona, ES); (2)Hospital Clínic (Barcelona, ES); (3)Hospital de la Santa Creu i Sant Pau (Barcelona, ES)

Introduction: The EBMT risk score has been widely validated in different settings. Recently, some modifications to the index have been suggested in order to improve its predictive capacity (Gratwohl. BMT. 2011). The modifications of the EBMT score deserve further validation in specific settings.

Methods: All patients receiving an allogeneic hematopoietic cell transplantation with reduced-intensity conditioning (allo-RIC) in two Spanish centers between 1999 and 2008 were included. RIC consisted of fludarabine in combination with melphalan $\left(70-140 \mathrm{mg} / \mathrm{m}^{2}\right)$ or busulfan $(8-10 \mathrm{mg} / \mathrm{kg})$. The median followup for survivors was 51 months (range 3-123) The classical EBMT score (cEBMT) was calculated as originally defined. The modified EBMT score (mEBMT) included an extra point for patients $>60$ years and neglected the time interval from diagnosis to transplant since its impact varies among hematological diseases.

Results: A total of 306 recipients (75\% from HLA identical siblings) were included. Most frequent diseases were AML/MDS $(n=99,32 \%)$ and NHL $(n=63,21 \%)$. The median cEBMT and mEBMT scores were 4 (range 2-7) and 4 (range 2-6). The probability of 100day non-relapse mortality (NRM), 4y-NRM and $4 y$-overall survival (OS) for the whole cohort were $13 \%(95 \% \mathrm{Cl}$ $10-16), 36 \%(95 \% \mathrm{Cl} 31-41)$ and $44 \%(95 \% \mathrm{Cl} 41-47)$, respectively. The only item from the score with significant impact on $\mathrm{NRM}$ and $\mathrm{OS}$ in the multivariate analysis was donor type (HR 1.7 [1.1-2.6], $p=0.01$ and HR 1.4 [1-2], $p=0.04$, respectively). Additionally, CMV negative patients transplanted from CMV negative donors showed higher OS (HR 0.5 [0.3-0.9], $p=0.03$ ) and lower NRM (HR 0.5 [0.2-1], $p=0.07$ ).

The $4 \mathrm{y}$ OS for the cEBMT categories were: score $2=46 \%$, $3=55 \%, 4=43 \%, 5=46 \%, 6=30 \%$ and $7=20 \% \quad(p=0.06)$, while applying the mEBMT were: score $2=62 \%, 3=53 \%, 4=40 \%$, $5=29 \%, 6=17 \%(p=0.001)$. NRM at 100 days using the mEBMT range from $5 \%$ (score 2$)$ to $48 \%$ (score 6$)(p=0.004)$ with steadily increasing HR for each additional score point, while the predictive capacity of the cEBMT was less accurate, specially for the lower scores $(2=10 \%, 3=7 \%, 4=9 \%)$. Both the cEBMT and the mEBMT showed similar predictive capacity for $4 y-N R M$. The addition of CMV serostatus to the scores did not improve their predictive capacity.

Conclusion: In the allo-RIC setting, both the cEBMT and mEBMT predict NRM and OS although the latter seems better. The addition of other items (like CMV status) to the score needs further evaluation in large registry-based studies. 


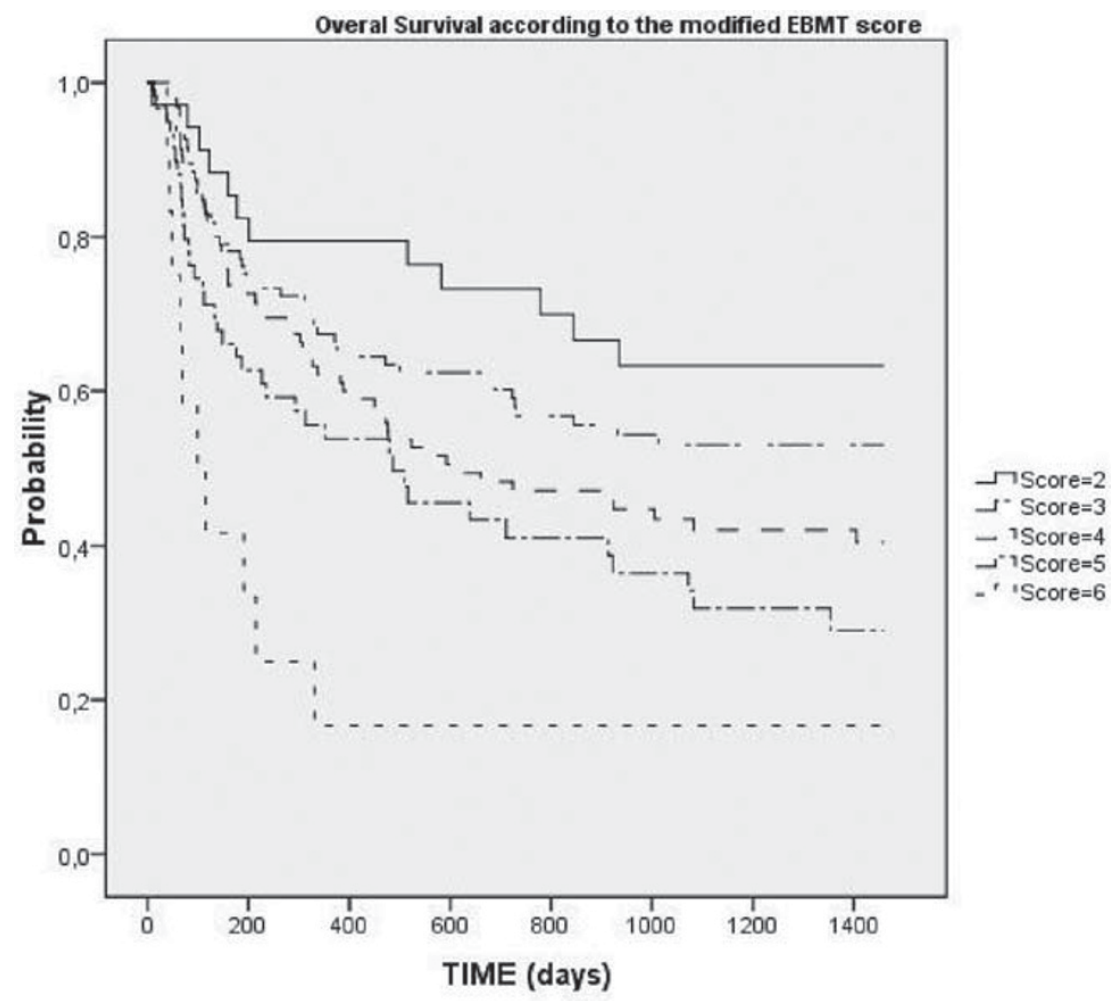

0149

Optimising patient selection for allogeneic stem cell transplantation: prediction of non-relapse mortality and survival

J. Pavlu, K. Rezvani, B. Kishore, J. Chakrabartty, R. Shah, H.W. Auner, D. Milojkovic, D. Marin, A. Rahemtulla, D.H. MacDonald, E.J. Kanfer, F. Dazzi, J.M. Goldman, J.F. Apperley, R.M. Szydlo Hammersmith Hospital (London, UK)

Introduction: It is important to identify prognostic indicators which may predict outcome of hematopoietic stem cell transplantation (HCT). Preconditioning serum level of C-reactive protein (CRP) was recently established as a novel prognostic factor in patients transplanted for CML in 1st chronic phase.

Patients and Methods: In this study we tested the value of CRP together with other prognostic factors in 256 patients who underwent myeloablative HCT for AML, ALL, MDS and advanced stage CML from February 1993 to April 2011. Recipients of sibling stem cells $(121,47 \%)$ received cyclophosphamide \& TBI conditioning. In unrelated transplants (135, $53 \%$ ) in vivo T-cell depletion with anti CD52 antibody was used in addition. Serum CRP levels (normal range 0-9 mg/ $\mathrm{L}$ ) were measured at a median of 11 days before stem cell infusion whilst patients were well and off antibiotics. Patients' comorbidities were defined and assigned weights (1-3) by the HCT comorbidity index ( $\mathrm{HCT}-\mathrm{Cl})$ and disease stage was assessed in accordance with EBMT criteria with 72 patients $(28 \%)$ classified as early, $139(54 \%)$ as intermediate and 45 $(18 \%)$ as late stage.

Results: In univariate analysis, factors associated with day 100 nonrelapse mortality (NRM) were recipient age and CMV status, disease stage, HCT comorbidity index ( $\mathrm{HCT}-\mathrm{Cl})$, and preconditioning CRP level. Multivariate analysis showed that recipient CMV IgG positivity, late disease stage, $\mathrm{HCT}-\mathrm{Cl}>2$ and elevated levels of CRP ( $>9 \mathrm{mg} / \mathrm{L}$ ) independently predicted increased NRM. Based on the relative risks of these 4 factors we developed a 4-point score for prediction of NRM (Figure). Patients scoring 0 points had a probability of NRM of $4 \%(95 \%$
Cl: 1-16), those with 1 point $14 \%$ (95\% Cl: 9-23), with 2 points $29 \%$ (95\% Cl: $19-44)$ and with $3-4$ points $60 \%$ (95\% Cl:42-86). Factors associated with survival in univariate analysis were: type of donor (sibling versus unrelated), disease stage, HCT$\mathrm{Cl}$, and preconditioning CRP level. In multivariate analysis only

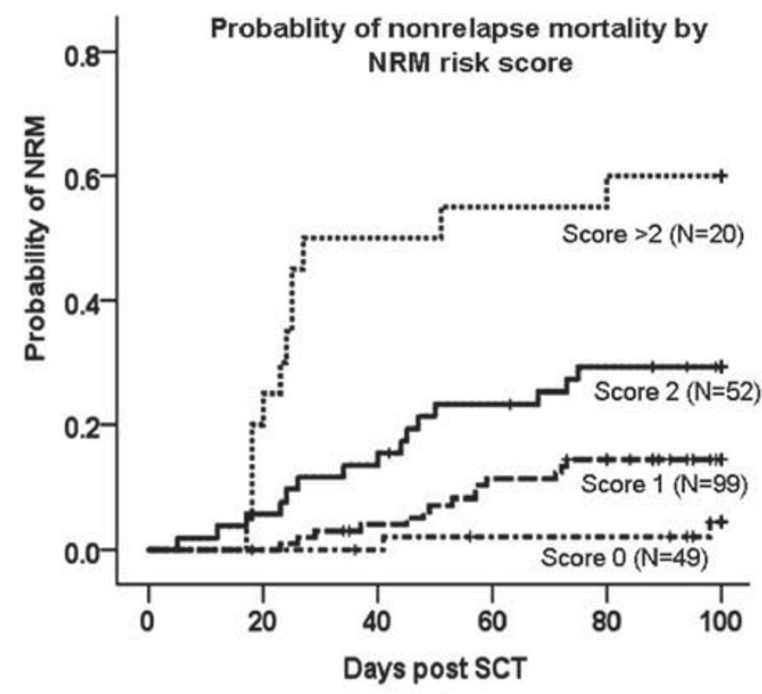

\begin{tabular}{lccc}
\hline Variable & RR & $95 \% \mathrm{Cl}$ & points \\
\hline CMV pos recipient & 2.9 & $(1.4-5.9)$ & 1 \\
Late disease stage & 2.9 & $(1.5-5.5)$ & 1 \\
HCT-Cl >2 & 2.9 & $(1.3-5.1)$ & 1 \\
Elevated CRP & 2.7 & $(1.5-5.0)$ & 1 \\
\hline
\end{tabular}


late disease stage, HCT-Cl>2 and elevated levels of CRP ( $>9$ $\mathrm{mg} / \mathrm{L}$ ) independently predicted inferior survival. Probabilities of 5 -year and 10-year survival in patients without any of these factors were 53 and $47 \%$ compared to 27 and $23 \%$ in patients who had one or more of the factors.

Conclusion: These results further establish preconditioning levels of CRP as a key prognostic variable for allogeneic HCT outcomes, and together with other well documented factors, may significantly aid the selection of patients who could benefit from HCT.

\section{0}

Age-adjusted recipient pre transplant telomere length and treatment-related mortality after haematopoietic stem cell transplantation

R. Peffault de Latour (1), R. Calado (2), M. Busson (1), J. Abrams (2), M. Robin (1), J. Larghero (1), A. Xhaard (1), N. Dhedin (1), E. Clave (1), D. Charron (1), A. Toubert (1), P. Loiseau (1), G. Socie (1), N. Young (2)

(1)Hôpital Saint Louis (Paris, FR); (2)National Institutes of Health (Bethesda, US)

Telomere attrition induces cell senescence and apoptosis. Dyskeratosis congenita, an inherited type of aplastic anemia caused by mutations in the telomerase complex genes and accelerated telomere shortening, is associated with high treatment-related mortality (TRM) after hematopoietic stem cell transplantation (HSCT). We hypothesized that age-adjusted pre-transplant telomere length predicted TRM after HSCT. Between 2000 and 2005, 178 consecutive patients underwent HSCT from HLA-identical sibling donors after myeloablative conditioning regimens ( $\mathrm{TBI}$ in 57 patients), mainly for hematological malignancies $(n=153)$, all performed in a single center. The stem cell source was bone marrow (BM) in 128 cases and peripheral blood (PB) in 50 cases. Median age at transplant was 32 years (range 3-65). Graft-versus-host disease (GvHD) prophylaxis mostly consisted of cyclosporine and methotrexate $(n=149$, $84 \%$ ). Before HSCT, blood lymphocytes were obtained from each donor-recipient pair. Telomere length was measured by real time quantitative PCR. We determined the normal age distribution of telomere length using a group of $17^{\star} 3$ healthy French hematopoietic stem cell donors $\left(f=-0.00833^{*}\right.$ age +1.522$)$ as a control group. We then calculated the pre-transplant recipient age-adjusted telomere length in comparison to controls. Ageadjusted pre-transplant telomere lengths were analyzed for correlation with clinical outcomes post HSCT using competing risk in univariate and multivariate analyses (Fine and Gray). After age-adjustment, patients' telomere length distribution was similar among all 4 quartiles except for disease severity. There was no correlation between telomere length and engraftment, GvHD, or relapse. The overall survival was $62 \%$ at 5 years (95Cl 54\%-70\%). After a median follow-up of 51 months (range,

Figure 1: Cumulative Incidence of Treatment Related Mortality (TRM) according to age-adjusted pre-transplant recipients' telomere length

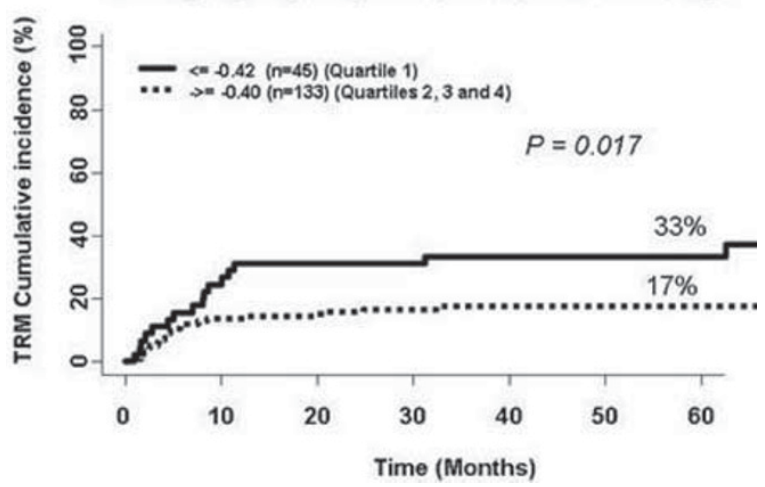

1-121 months), 43 patiens had died due to TRM. The TRM rate inversely correlated with telomere length. TRM in patients in the first (lowest telomere length) quartile was significantly higher than in patients with longer telomeres $(p=0.017)$ (Figure 1). In multivariate analysis, age of the recipients (HR: 1.1, 95\% $\mathrm{Cl}[.0-1.1, p=0.0001]$ and age-adjusted telomere length [HR: $0.4,95 \% \mathrm{Cl}[0.2-0.8, \mathrm{p}=0.01])$ were independently associated with TRM. No association was found between donor telomere length and outcome post HSCT. In conclusion, age-adjusted recipient pre-transplant telomere length is an independent biological marker of TRM after HSCT.

0151

Liver iron content - as determined by magnetic resonance imaging - is an independent predictor for early non-relapse mortality in MDS and AML patients undergoing allogeneic stem cell transplantation M. Wermke, A. Schmidt, M. Middeke, K. Sockel, M. von Bonin, V. Plodeck, M. Laniado, J. Schetelig, G. Ehninger, M. Bornhäuser, U. Platzbecker

University Hospital (Dresden, DE)

Systemic iron overload (SIO) has been described as an adverse prognostic factor in patients undergoing allogeneic stem cell transplantation (allo-SCT). However, almost all existing studies relied on interference prone surrogate markers like ferritin and reached a variety of different conclusions. Further, optimal surrogate parameter thresholds for risk stratification remain to be defined. We aimed at assessing the diagnostic and prognostic impact of liver iron content (LIC) measured by magnetic resonance imageing (MRI) in a cohort of $81 \mathrm{AML}$ and MDS patients undergoing allo-SCT.

MRI-based assessment of LIC was included into the routine workup of all our AML and MDS patients undergoing allo-SCT. Correlations between serological parameters and LIC were assessed by Spearmans rank correlation coefficient ( $r$ ). Categorial variables were compared using Fisher's excact test. Post-transplant outcomes including the occurrence of GVHD as well as NRM and overall survival (OS) were analyzed by means of competing events statistics and Kaplan-Meier estimates, respectively.

Both ferritin and RBC count were significantly $(p<0.001)$ correlated with LIC ( $r=0.594$ and $r=0.702$, respectively). With areas under the receiver-operator characteristic of 0.784 and 0.823 both parameters seemed to be adequate predictors of a LIC $\geq 125 \mu \mathrm{mol} / \mathrm{g}$. While a threshold of $\geq 20 \mathrm{RBC}$ predicted for an elevated LIC with a sensitivity of $70.0 \%$ and a specificity of $81.8 \%$, a ferritin $\geq 1000 \mathrm{ng} / \mathrm{ml}$ albeit being very sensitive (95.5\%), provided only poor specificity $(27.0 \%)$. As a consequence we decided to increase the ferritin threshold to $2500 \mathrm{ng} / \mathrm{ml}$, which resulted in a much better balance between sensitivity $(63.6 \%)$ and specificity $(81.1 \%$. None of the three SIO parameters was associated with an increased risk of aGvHD and only LIC but not ferritin or a transfusion burden predicted for an increased cumulative incidence ( $\mathrm{Cl}$ ) of NRM (Figure 1). Indeed, multivariate analysis confirmed LIC as an independent risk factor for NRM (HR 1.008 for every $1 \mu \mathrm{mol} / \mathrm{g}$ increase, $p=0.005$ ). This translated into a shorter OS in patients with an LIC $\geq 125 \mu \mathrm{mol} / \mathrm{g}$ (Figure 2) which was confirmed in multivariate Cox-regression analysis (HR: $3.481, p=0.021$ ).

We conclude that MRI-based measurement of LIC but not ferritin is an independent negative prognostic factor for post-transplant outcome of AML and MDS patients. 


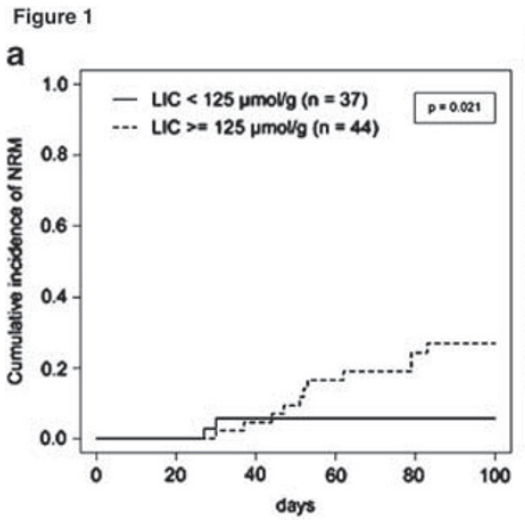

b

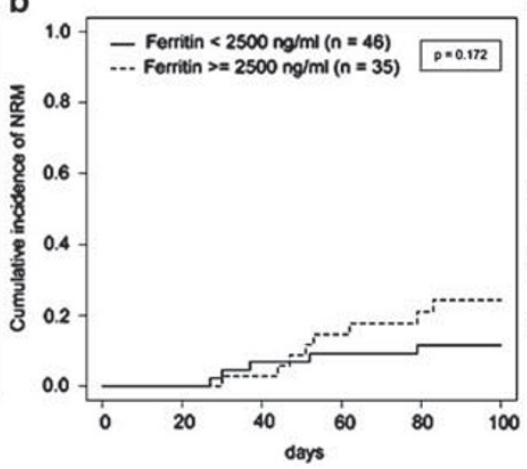

C

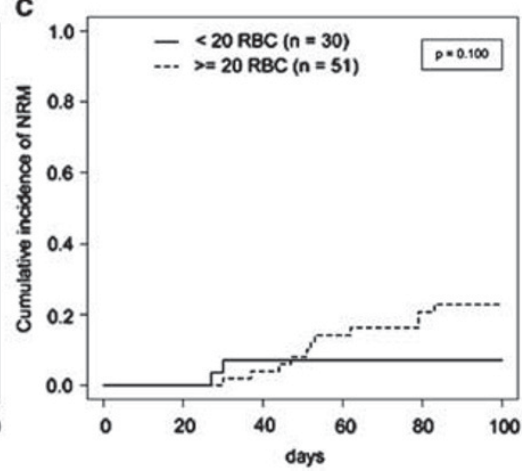

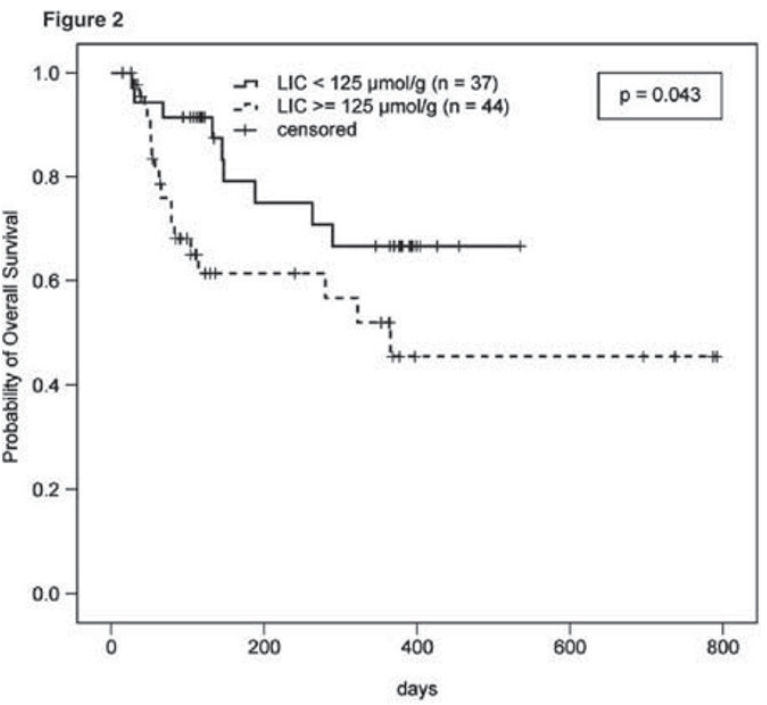

0152

Laboratory markers of endothelial dysfunction associated with early non-infectious complications and acute graft-versus-host disease in patients undergoing allogeneic haematopoietic stem cell transplantation

A. Czyz, J. Rupa-Matysek, A. Lojko-Dankowska, E. Wojtasinska, A. Mierzwa, K. Zajdel, A. Miedzinska, M. Komarnicki Poznan University of Medical Sciences (Poznan, PL)

Background: There are numerous data indicating that endothelial injury plays a central role in the activation of immune system following allogeneic haematopoietic stem cell transplantation (alloHCT) and precedes non-infectious complications of vascular origin and graft-versus-host disease (GvHD). The aim of the study was to evaluate a possible correlation between the changes of plasma endothelial dysfunction markers and the occurrence of early non-infectious complications and acute GvHD (aGvHD) after alloHCT.

Patients and Methods: Plasma levels of von Willebrand factor antigen (vWFAg), trombomodulin (TM) and activity of factor VIII (fVIII) were measured in 63 pts transplanted with allogeneic stem cells after myeloablative (43 pts) or reduced-intensity (20 pts) conditioning regimen for AML (26 pts), ALL (18 pts), myeloproliferative neoplasms (15 pts) and lymphoma (4 pts). Endo- thelial dysfunction markers were measured before conditioning regimen (day -10$)$, on the day of stem cells infusion (day 0 ) and on the day +10 and +30 after alloHCT.

Results: The TM level on the day 0 was significantly higher in the group of pts who developed early non-infectious complications ( $\mathrm{n}=7$ including 5 pts with multiorgan failure, 1 patient with VOD and 1 patient with IPS) compared to the group without those complications $(\mathrm{n}=56)(7,5 \mathrm{ng} / \mathrm{ml}$ vs $3,7 \mathrm{ng} / \mathrm{ml} ; \mathrm{p} 0.03)$ (Figure 1). The concentration of vWFAg was significantly elevated on the day $0,+10$ and +30 in comparison to the day -10 in the whole group of patients. However, the relative increase in the concentration of vWF Ag on the day +10 in comparison to the baseline concentration on the day -10 tended to be higher in the group of pts developing grade II-IV aGvHD thereafter $(n=18)$ compared to the group of pts without clinically significant aGvHD $(n=40)$ (mean relative increase in concentration 3,7 vs 1,$8 ;$ p 0.059).

Conclusions: The association between markers of endothelial dysfunction and the occurrence of early non-infectious complications and aGvHD after alloHCT was found. TM level on the day of stem cell infusion, that may reflect endothelial injury induced by conditioning regimen, is associated with the occurrence of early non-infectious complications. The relative increase in vWFAg concentration early after alloHCT tends to be associated with the development of aGvHD. Further studies evaluating the potential role of these markers as early predictors of non-infectious complications and aGvHD after alloHCT are warranted.

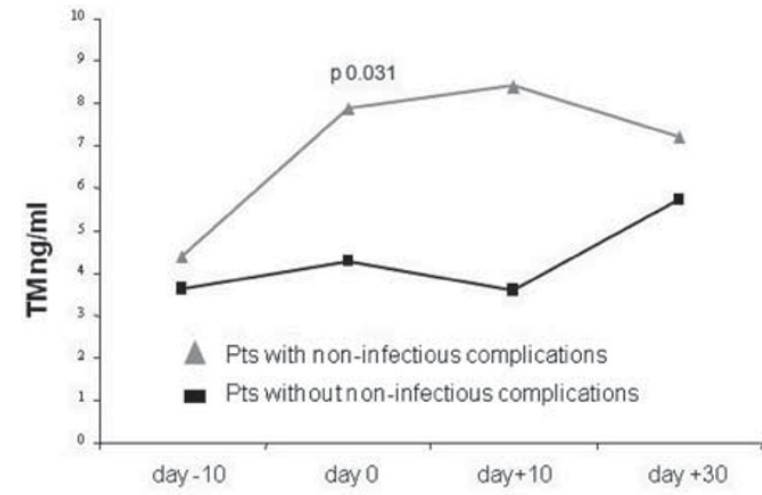

Figure 1. TM level before and after alloHCT 


\section{Minimal Residual Disease, Tolerance, Chimerism and Immune Reconstitution}

\section{3}

Thymosin alfa 1 administration improves immune reconstitution and decreases infection-related mortality after HLA-matched sibling T cell-depleted stem cell transplantation

L. Ruggeri (1), K. Perruccio (1), A. Pierini (1), S. Moretti (1), F. Topini (1), A. Tosti (1), A. Carotti (1), F. Aversa (1), M. Martelli (1), F. Bistoni (1), E. Garaci (2), L. Romani (1), A. Velardi (1) (1)University of Perugia (Perugia, IT); (2)Istituto Superiore di Sanità (Rome, IT)

Bone marrow transplanted mice show impaired dendritic cell (DC) function. Administration of Thymosin-alpha-1 (T-alpha-1), a naturally occurring peptide, induces DC activation, maturation and differentiation. It accelerates lymphoid cell recovery, promotes balanced Th1/Treg immunity and protects mice from invasive aspergillosis [Romani et al., BLOOD 2004, 103:42324239; Orabona et al., Blood 2006, 107:2846-2854]. We, therefore, designed a phase I/II clinical trial to determine the safety and efficacy of T-alpha-1 administration in 30 recipients of HLA-matched sibling T cell-depleted stem cell transplants (12/30 with active disease at transplant). Patients aged 20-69 years (median 46) with AML/MDS (12), ALL (6), Iymphoma (7), MM (4), CLL (1) were conditioned with TBI or Melphalan, Thiothepa, and Fludarabine (Aversa et al EBMT 2011) and given T alpha 1 (1.6 mg/day s.c.) from the day of transplant onwards for 16 weeks. Forty-five patients (25/45 with active disease at transplant), who were transplanted under the same protocol, served as controls. They were aged $20-67$ years (median 53 ) with AML/MDS (20), ALL (4), lymphoma (11), MM (6), CLL (3), myelofibrosis (1). T-alfa-1 administration caused no adverse effects whatsoever. Immune reconstitution was assessed by limiting dilution analyses of frequencies of CD4+ $T$ cells that were specific for Aspergillus, Candida, Cytomegalovirus, Adenovirus, Herpes Simplex Virus, Varicella- Zoster Virus, Toxoplasma antigens. Normal donor values ranged from 600 to 1200/10e6 plated cells. Control transplant recipients acquired such pathogen-specific $T$ cell responses from month 3 onwards in frequencies that ranged from 50 to $250 / 10$ e 6 plated cells. In patients who received $T$ alfa 1 , pathogen-specific $T$ cells appeared as early as 1 month after transplant in significantly higher frequencies which soon ranged from 250 to 500/10e6 plated cells. The cumulative incidence of non-relapse mortality (NRM) (mainly infection-related) was 33\% in controls vs $10 \%$ in Thymosin-treated patients $(p=0.02)$. As a consequence of the improved TRM, Event-Free Survival was better in Thymosin treated patients $(41 \%$ vs $21 \%$ in controls; $p=0.06)$. Finally, multivariable analyses that included diagnoses, disease status at transplant, conditioning regimen and donor lymphocyte infusions showed Thymosin treatment was a significant independent factor predicting a lower incidence of NRM $(p=0.04)$ which tended to provide better survival $(p=0.1)$.

\section{4}

Adoptive immunotherapy with Tregs and Tcons promote a GvL effect in absence of GvHD in HLA-haploidentical transplantation

M. Di lanni, F. Falzetti, A. Carotti, A. Terenzi, A. Pierini, M.S. Massei, A. Velardi, F. Aversa, M.F. Martelli

University of Perugia (Perugia, IT)

In HLA-haploidentical stem cell transplantation we showed adoptive immunotherapy with naturally occurring $T$ regulatory cells (nTregs) followed by conventional T cells (Tcons) preven- ted acute and chronic graft-versus-host-disease (GvHD), favoured lymphoid reconstitution and immunity against pathogens (Di lanni et al., Blood 2011). A major concern is whether a graftversus-leukemia (GvL) effect is maintained since FoxP3+Tregs can also suppress immune response to tumour. 49 patients (39 AML, 8 ALL, 1 Biphenotipic AL, $1 \mathrm{NHL}$ ) received a conditioning regimen including $\mathrm{TBI}$, thiotepa, fludarabine and cyclophosphamide (28 patients in the 1st clinical trial) or alemtuzumab (21 patients in the 2 nd clinical trial), followed by an infusion of nTregs (day -4 ; mean $2.6 \times 10^{6} / \mathrm{kg} \pm 0.8 \mathrm{SD}$; FoxP3+ cells $92 \% \pm 8 \mathrm{SD}$ ) and on day 0 CD34+ cells (mean $9.8 \times 10^{6} / \mathrm{kg} \pm 3.8$ $\mathrm{SD}$ ) and Tcons (mean $1.1 \times 10^{6} / \mathrm{kg} \pm 0.5 \mathrm{SD}$ ). No post-transplant GvHD prophylaxis was given. $47 / 49$ patients achieved a rapid and sustained full donor-type engraftment. 5/45 (11\%) valuable patients developed acute GvHD. CD4+ and CD8+ peripheral blood counts reached $200 / \mu \mathrm{L}$ on days 70 (range 37-189 days) and 51 (range 21-147) respectively. We observed a rapid development of a wide T-cell repertoire and high frequencies of specific CD4+ and CD8+ clones for opportunistic pathogens. Treg immunotherapy did not compromise posttransplant generation of donor-vs-recipient alloreactive natural killer (NK) cell repertoires which was faster than controls and with an enhanced alloreactivity against KIR-ligand mismatched targets. The cumulative incidence of relapse was 0.076 as only 3 high risk patients have relapsed to date at a median follow-up of 16 months (range 4-37). Preservation of alloreactive NK cell repertoires plays a key role in controlling leukaemia relapse. However more than $50 \%$ of our patients were transplanted from a non NK alloreactive donors, so we can hypothesize that high numbers of Tcons in the absence of post-transplant immunosuppression will also exert a GvL effect.

\section{5}

Mixed chimerism, minimal residual disease and immunotherapy after haematopoietic stem cell transplantation for acute lymphoblastic leukaemia in children

G. Lucchini (1), H. Kreyenberg (2), A. Jarisch (2), J. Sörensen (2), C. Peters (3), A. Willasch (2), E. Rettinger (2), S. Kuci (2), U. Köhl (2), T. Klingebiel (2), P. Bader (2)

(1) University of Milano Bicocca (Monza, IT); (2)University of Frankfurt (Frankfurt am Main, DE); (3)St. Anna Children Hospital (Vienna, AT)

Objective: Mixed chimerism (MC) after stem cell transplantation (SCT) for acute lymphoblastic leukemia (ALL) is known to predict relapse of the original disease in most cases. Immunotherapy (IT), including immunosuppressive withdrawal and donor lymphocytes infusion (DLI), is a possible strategy to prevent relapse in patients (pts) with MC. The aim of the present study is to analyse the outcome of a pediatric cohort of pts receiving SCT for ALL according to their chimerism status. Moreover, the impact of IT in pts with MC and the relationship between MC and minimal residual disease (MRD) after SCT are described. Patients and Methods: 68 pts diagnosed with ALL received SCT from matched related or unrelated donors after myeloablative conditioning (TB and VP16) at the University Children's Hospital in Frankfurt, between Jan 1st, 2005 and Dec 31st, 2010. Chimerism analysis was performed using STR based quantitative PCR. Investigations were performed weekly until day 200 and monthly thereafter. 30 pts $(44.2 \%)$ presented MC after day +30 from SCT. All children with $\mathrm{MC}$ were pre-emptively treated by immunosuppression withdrawal, eventually followed by DLI administration.

Results: The whole cohort had a probability of event free survival ( $p E F S$ ) of $75 \%$ and a probability of overall survival (pOS) of $80.9 \%$. Transplant related mortality in the all cohort was $13.2 \%$. Pts with persistent full donor chimerism had a pEFS of $92.1 \%$ while those with MC achieved a pEFS of $40.6(p=0.001)$. All patients with MC could be pre-emptively treated; $19 / 30$ patients $(=63 \%)$ converted to complete chimerism and remained in CR. 10 out of 22 relapsed after withdrawal of immunosuppression. 
8 pts received further DLI. 7 out of these 8 pts responded and remain in $\mathrm{CR}$ whereas 1 patient relapsed.

No severe GvHD and no cytopenia were reported after DLI administration. MRD data from bone marrow samples after SCT were available for 44 pts. 25 pts were MRD negative, among which 1 relapsed with isolated extra-medullary disease while exhibiting full donor chimerism. 8 pts had low MRD levels $\left(<1 \times 10^{-4}\right)$ and none of them relapsed. 11 pts were MRD positive $\left(\geq 1 \times 10^{-4}\right)$ and exhibited MC, 9 of them relapsed, in 7 cases MRD positivity preceded MC detection in bone marrow. Conclusions: Consequent analysis of chimerism offers the possibility to identify pts at highest relapse risk. Even though the numbers of this cohort are small to draw firm conclusions, preemptive immunotherapy may positively influence outcome.

\section{6}

Low incidence of viral complications after in vivo and in vitro T-cell depletion using low dose alemtuzumab is due to early post transplant outgrowth of protective virus specific CD4 and CD8 memory T-cells by various escape mechanisms

C. Halkes, J.H.F. Falkenburg, E. van Egmond, M. van Meent, P. von dem Borne, W.A.F. Marijt, J.H. Veelken, I. Jedema LUMC (Leiden, NL)

Despite profound T-cell depletion by in vitro $(20 \mathrm{mg})$ and in vivo (30 mg) Alemtuzumab (ALT) for acute GvHD prevention, no early CMV disease and a low incidence of $2 \%$ of EBV associated PTLD was seen in a cohort of 41 patients receiving allogeneic stem cell transplantation (alloSCT) after a reduced intensity, fludarabin and busulphan based conditioning regimen. We hypothesized that virus specific memory $T$ cells are capable of surviving circulating ALT. In this study we analyzed the recovery of CD4 and CD8 T cells and CMV and EBV specific memory cells in relation to circulating antibody levels. Three weeks after alloSCT, low numbers of circulating CD8 and CD4 T cells were found (median 0.6 and $0.3 \mathrm{M} / \mathrm{L}$, respectively). These numbers increased after 6 weeks to 27.6 CD4 and 9.4 CD8 T cells $\mathrm{M} / \mathrm{L}$, and after 9 weeks to $61.5 \mathrm{CD} 4$ and $19.0 \mathrm{CD} 8 \mathrm{~T}$ cells $\mathrm{M} / \mathrm{L}$. At these time points circulating ALT was found in most patients (median levels $0.39,0.05$ and $0.02 \mathrm{ug} / \mathrm{ml}$, after 3,6 and 9 weeks, respectively). Six weeks after alloSCT in $28 / 29$ patients circulating CD4 T cells lacking CD52 expression were present (median $69 \%$ of CD4 cells, range $0-93 \%$ ) and in $27 / 29$ patients CD52 negative CD8 T cells were found (median $46 \%$ of CD8 cells, range $0-99 \%$ ). Based on concurrent FLAER negativity, we demonstrated that the CD52 negative T cells were GPI anchor deficient. Because we also detected significant numbers of circulating CD8 T cells with CD52 expression despite functional levels of circulating ALT, we examined CD52 expression levels and in-vitro sensitivity to ALT-mediated complement-dependent cytotoxicity (CDC) of CD4 and CD8 T cells. CD52 expression on CD8 $T$ cells was lower than on CD4 T cells, resulting in relative protection of CD52 positive CD8 T cells against ALTmediated CDC. Using tetramer staining, cytotoxicity assays and analysis of cytokine production, we demonstrated that both CD52 negative and CD52 positive CMV and EBV specific CD8 $T$ cells were functional.

In conclusion, after TCD using low dose ALT, virus specific memory $T$ cells are able to survive due to various escape mechanisms including CD52 negative GPI deficient escape variants in both the CD4 and CD8 compartments. Moreover, a proportion of CD8 T cells was capable of escaping from ALTinduced CDC due to lower expression of CD52 as compared to CD4 T cells. All escape variants contained fully functional virus-specific $T$ cells, which may explain the low incidence of viral complications after low dose ALT-induced TCD.
0157

HY-specific regulatory T-cells and male microchimerism are established in both parous and nulliparous females A.G.S. vanHalteren (1), M.P.Dierselhuis(1), E. Jankowska-Gan(2), E. Blokland (1), J. Pool (1), W.J. Burlingham (2), E. Goulmy (1) (1)Leiden University Medical Center (Leiden, NL); (2)University of Wisconsin (Madison, US)

The influence of donor parity on the outcome of HLA matched hematopoietic stem cell transplantation (HSCT) is poorly understood. Through exchange of hematopoietic and nonhematopoietic cells, pregnancy frequently leads to the stable engraftment of small numbers of fetal cells; these blood-borne or tissue-residing microchimeric cells may be accompanied by functionally different types of maternal alloreactive $T$ cells, including minor Histocompatibility antigen-specific cytotoxic (T-CTL) or regulatory $T$ cells (T-REG). We investigated whether male microchimerism detectable in peripheral blood cells collected after the delivery of male offspring is correlated with HY-specific regulatory T cells activity in 57 healthy women with a well documented obstetric and family history. A real-time quantitative polymerase chain reaction was used to determine the presence of a $\mathrm{Y}$ chromosome-specific multicopy locus (DYS-1) in 5 different highly purified hematopoietic cell types, i.e. T cells, B cells, monocytes, myeloid dendritic cells and/or granulocytes. The trans-vivo Delayed Type Hypersensitivity reaction was used to determine the presence or absence of $\mathrm{HY}$ peptide-specific T-REG in the same peripheral blood sample. $\mathrm{Y}$ chromosome positive leucocytes were detected in 16 out of 35 women with male offspring, but also in 4 out of 8 donors with female offspring and in 4 out of 14 nulliparous females. T-REG specific for HY peptides presented by HLA class II molecules were detected in 10 out of 24 women; their HLA class I-restricted counterparts were identified in 17 out of 32 donors analyzed. These presumably CD4 or CD8 positive T-REG were detected irrespective of the presence or absence of circulating male microchimeric cells. Our study indicates that HY-specific T-REG appear to be a common occurrence in healthy women, irrespective of their pregnancy history. It remains to be studied whether blood-borne male cells, tissue-residing male cells or both are necessary for the induction and maintenance of this male-specific tolerance.

\section{8}

Significant expansion of myeloid and lymphoid HLA-G+ immunosuppresive cells after allogeneic haematopoietic cell transplantation in blood and in GvHD-affected skin I. Lazana, A. Zoudiari, D. Kokkinou, M. Liga, A. Spyridonidis University Hospital Patras (Rio / Patras, GR)

Introduction: The human leukocyte antigen-G (HLA-G) is an important tolerogeneic molecule, initially shown to mediate maternal tolerance of the "semi-allogeneic" fetus during pregnancy. We have previously identified a small subset of HLA$\mathrm{G}+\mathrm{T}$ cells and a sizable population of HLA-G+ monocytic cells (CD14+/CD16low/HLA-DRlow) circulating in peripheral blood (PB) of healthy individuals with potent immunoregulatory and immunotolerogeneic properties.

Objectives: To investigate the evolution of naturally occurring HLA-G+ myeloid (CD14+) and lymphoid (CD3+) populations after allogeneic hematopoietic cell transplantation (HCT).

Methods: PB from 27 unselected transplant patients were analyzed for the presence of HLA-G+ cells by flow cytometry. FACS sorted HLA-G+ cells were used as third party cells in mixed lymphocyte cultures (MLC) and CFSE cell proliferation assays to analyze their immunosuppressive properties. Results: HLA$\mathrm{G}+$ cells in PB of patients after HCT $(8.1 \% \pm 0.8)$ was significantly increased, as compared to healthy donors (mean 2.5 -fold, $p<0.0001$ ) or to respective percentages before HCT (mean 2.8-fold, $\mathrm{p}=0.01$ ). Interestingly, there was a more pronounced expansion of CD3+HLA-G+ cells (5.4 fold increase) as compared to that of CD14+HLA-G+ cells ( 2.2 fold increase). 
CD3+HLA-G+ cells were at median $8.7 \% \pm 0.8$ in transplant patients, as compared to both healthy subjects $(1.6 \pm 0.4, p<0.0001)$ or pre-transplantation values $(1.1 \pm 0.2, p=0.005)$. By delineating patients according to the time after transplantation, we found that HLA-G+ cells, both CD3+ and CD14+, were detected early after transplantation $(\mathrm{d}<100)$ and continued to be high at later time periods $(100<d<365, d>365)$. In allogeneic MLC assays, FACS-sorted CD3+HLA-G+ and CD14+ HLA-G+ cells from transplanted patients were able to suppress $\mathrm{T}$-cell responses $(p=0.002$ and $p=0.0005$, respectively). Although in our study we couldn't find any correlation between graft-versus-host disease (GvHD) and PB HLA-G+ cells, interestingly, HLA-G+ cells were detected in significant amounts in skin biopsies and their frequency correlated with GvHD $(p=0.03)$.

Conclusions: Overall, we believe that HLA-G+ cells belong to the normal repertoire of the immune system and after allo-HCT increase acting as a shield against immune aggression, especially in target organs. Since these cells are easy to isolate from blood, naturally occurring HLA-G+ cells may constitute a novel strategy for adoptive cell immunosuppressive therapy.

\section{9}

Longitudinal follow-up of minimal residual disease in aml after SCT with quantitative evaluation of WT1 gene expression

A. Candoni, E. Simeone, C. D'Odorico, E. Toffoletti, R. Fanin University of Udine (Udine, IT)

Introduction: Wilms Tumor gene (WT1) overexpression is described in several oncological diseases including acute myeloid (AML) leukemias. The majority of AML patients don't have a suitable specific molecular marker for monitoring minimal residual disease (MRD). Quantification of WT1 in bone marrow samples can be useful as a marker of MRD after allogeneic stem cell transplantation (SCT) and can predict AML relapse. Methods and Results: We have evaluated, sequentially and using a quantitative RT-PCR technique (Leukemia NET method), WT1 expression in 50 consecutive AML patients that overexpressed WT1 at diagnosis and that underwent allogeneic SCT at our centre. The cDNA level of WT-1 was detected in bone marrow samples at diagnosis at the time of transplant and after the allogeneic SCT. Samples of diagnosis showed high WT1 expression levels in all cases with a mean of 5570 (SD 4055) copies of WT1/10000 Abl, median 4600 (range 65823913) copies WT1/10000 Abl. At transplant 34 pts (68\%) were in complete cytologic remission (CcR) and 16 (32\%) had refractory or relapsed AML. Bone marrow samples from pts in $\mathrm{CcR}$ at BMT showed significantly lower WT1 expression levels (mean $88 \pm 130$ ), compared to the samples from pts with relapsed or refractory disease (mean $5727 \pm 4265)(P<0,001)$. After BMT a rapid decline of WT1 expression levels was observed in all pts that achieved and/or maintained a condition of CcR, especially in those that were in CcR at SCT. After a median follow up of 11 mths from transplant, 10 out 50 pts relapsed $(20 \%)$ and all of them had high expression levels of WT1 before the cytological relapse. Three of these pts were successfully reinduced with $\mathrm{DLI} \pm$ chemotherapy with a rapid reduction of WT1 levels. Conclusions:

1) In our experience there is a complete concordance between WT1 expression levels (measured by quantitative RT-PCR) and status of AML before and after SCT.

2) Our study confirms that longitudinal quantitative evaluation of WT1 after SCT may be useful as a non-specific leukemia marker (NSLM) for monitoring MRD and as a predictor of AML relapse.

3) Based on these results cases with an increase of WT1 levels after SCT and without GVHD should be candidate to DLI and/or discontinuation of immunosuppressive therapy.

\section{Working Party Session SAA}

160

\section{Chairman's report}

J. Marsh (London, UK)

\section{Scientific activity report}

1. Introduction: The Severe Aplastic Anaemia Working Party (SAAWP) reports on AA and other rare acquired and inherited bone marrow failure disorders (see Figure 1). The AA database is the only "disease specific" database within the EBMT as data are not only collected on patients receiving transplant but also other forms of therapy, including immunosuppressive therapy.

2. Major scientific successes.

The prospective clinical trial of rabbit ATG (Thymoglobulin) NCT00471848 was completed in 2011 and the manuscript is about to be submitted.

Oral presentation given at the Annual 2011 American Society for Haematology (ASH) meeting: de Latour, Long-Term Outcome After Matched Allogeneic Hematopoietic Stem Cell Transplantation for Fanconi Anemia (Blood 2011; 118: 325).

3. The most relevant activities in 2011 :

i. Joint Educational Meeting of SAA, Late Effect and Autoimmune Diseases WPs, 3-6th November 2011 held in Barcelona. ii. The complete treatment algorithm for SAA which was placed on the EBMT website last year has been accepted as an invited document to be published as a special issue of the journal Bone Marrow Transplantation.

4. Important objectives for 2012:

i. To promote further international collaborations with, for example, Centre for Blood and Marrow Transplantation Research (CIBMTR).

ii. To organise a second joint education meeting of SAA, Late Effects and Autoimmune Diseases WPs for 2012.

iii. To initiate a new clinical trial in aplastic anaemia. Indicators/key figures:

1. Budget: EUR 24,190

2. Sponsors:

Genzyme Therapeutics for Rabbit ATG (Thymoglobuline) study, £333,291.

3. Number of education activities: one for 2011 and a further one planned for 2012 (see above).

4. Number of publications and impact number: 3 published manuscripts, 2 submitted, 8 in preparation. Total impact factor of the 3 published manuscripts $=54.749$ (with Haematologica manuscript in press $=61.281$ ).

5. ASH 2011 Presentations: oral presentation, $n=1$; poster presentations, $n=3$.

6. EBMT 2011 Presentations: oral presentations, $n=7$; poster presentations, $n=1$.

7. Number of studies:

i. Clinical Trials: (a) Prospective phase II study of Rabbit ATG with ciclosporin for patients with AA. Study closed and manuscript about to be submitted.

ii. Retrospective studies: 19 in progress.

List of Publications:

i. Tichelli A, Schrezenmeier H, Socie G, Marsh J, Bacigalupo A, Duehrsen U, Franzke A, Hallek M, Thiel E, Wilhelm M, Hoechsmann B, Barrois A, Champion K, Passweg J. A randomized controlled study in newly-diagnosed severe aplastic anemia patients receiving antithymocyte globulin, ciclosporin, with or without G-CSF. Blood First Edition Paper, prepublished online January 13, 2011; DOI: 10.1182/blood-2010-08-304071.

ii. Eapen M, Le-Rademacher J, Antin J, Champlin RE, Carreras J, Fay J, Passweg JR, Tolar J, Horowitz MM, Marsh JC, and Deeg HJ. Effect of stem cell source on outcomes after adult unrelated donor transplantation in severe aplastic anemia. Blood 2011 blood-2011-05-354001; published ahead of print June 15, 2011, doi:10.1182/blood-2011-05-354001.

iii. European Blood and Marrow Transplant Group, Severe Aplastic Anaemia (Dufour C, Bacigalupo A, Socie G, Tichelli A, 
Risitano AM, Schrezenmeier H, Locasciulli A, Aljurf M, Oneto R, Passweg JR, Marsh JC). Rabbit ATG for aplastic anaemia treatment: a backward step? Lancet (Comment) Published Online July 6, 2011, DOI: 10.1016/S0140-6736(11)60817-9.

Total Impact Factor score $=61.28$.

Manuscripts submitted:

i. Bacigalupo A, Socié G, Schrezenmeier H, Tichelli A, Locasciulli A, Fuehrer M, Risitano AM, Dufour C, Passweg JR, Oneto R, Aljurf M, Flynn C, Mialou V, Hamladji RM and Marsh JCW for the Aplastic Anemia Working Party of the European Group for Blood and Marrow Transplantation (WPSAA-EBMT) Bone marrow versus peripheral blood sibling transplants, in acquired aplastic anemia: survival advantage for marrow in all age groups. Haematologica 2012, in press.

ii. R. Peffault de Latour, H. Schrezenmeier, A. Bacigalupo, D. Blaise, C.A. de Souza, S. Vigouroux, R. Willemze, L. Terriou, A. Tichelli, M. Mohty, S. de Guibert, J.C. Marsh, J. Passweg J.Y. Mary, G. Socié, on behalf of the Severe Aplastic Anemia Working Party (SAA WP) of the European Group for Blood and Marrow Transplantation (EBMT) and the French Society of Hematology (SFH). Allogeneic Stem Cell Transplantation in Paroxysmal Nocturnal Hemoglobinuria. Blood submitted.

Manuscripts in preparation: $n=9$ :

i. Rabbit antithymocyte globulin (rATG) and ciclosporin for patients with aplastic anaemia: an EBMT prospective study (Marsh et al). To be submitted to Lancet.

ii. The complete treatment algorithm for SAA. Invited special edition of BMT journal (Aljurf et al).

iii. Aplastic anaemia in adolescents, (Dufour et al).

iv. Haploidentical HSCT in acquired SAA (MT Staghellini et al). v. Outcomes of transplants from syngeneic donors in patients with aplastic anaemia (Gerull et al).

vi. Outcomes of truly refractory severe acquired AA (Rovo et al). vii. Retrospective study on outcome of a second unrelated HSCT for SAA patients (Cesaro et al).

viii. HSCT in dyskeratosis congenita, combined EBMT/CIBMTR study, (Korthof et al).

ix. Unrelated stem cell transplantation for acquired aplastic anemia: a donor versus no-donor analysis Sébastien Maury et al. Meeting abstracts:

1. ASH 2011 oral presentation:

i. Regis de Latour, Long-term outcome after matched allogeneic hematopoietic stem cell transplantation for Fanconi Anemia on behalf of the FA Committee of the Severe Aplastic Anemia Working Party (SAA WP) and the Pediatric Working Party of the European Group for Blood and Marrow Transplantation (EBMT). Blood (ASH Annual Meeting Abstracts), Nov 2011; 118: 325.

2. ASH 2011 posters:

i. De Latour et al. Allogeneic Hematopoietic Stem Cell Transplantation in Paroxysmal Nocturnal Hemoglobinuria: A Transplant Versus No Transplant Matched Comparison Study On Behalf of the Severe Aplastic Anemia Working Party (SAA WP) of the European Group for Blood and Marrow Transplantation (EBMT) Group and the French Society of Hematology (SFH). Blood 2011; 118: 2403.

ii. Marsh et al, Prospective Phase II Pilot Study of Rabbit Antithymocyte Globulin (ATG, Thymoglobuline) with Ciclosporin for Patients with Acquired Aplastic Anemia and Matched Pair Analysis with Patients Treated with Horse ATG (Lymphoglobuline) and Ciclosporin: A Study From the EBMT Severe Aplastic Anemia Working Party (RATGAA07). Blood 2011; 118: 2408 .

iii. Korthof et al. Dyskeratosis Congenita: Evaluation of Immune Status and Hematopoietic Stem Cell Transplantation. A Literature and EBMT Data Base Survey of 75 Patients. Blood (ASH Annual Meeting Abstracts), Nov 2011; 118: 4144.

3. EBMT 2011 oral presentations:

i. J. Passweg, G. Socié, A. Tichelli, P.A. von dem Borne, A. Schattenberg, R. Foa, A. Ganser, C. Dufour, A. Bacigalupo, A. Locasciulli, M. Aljurf, C. Peters, A. van Biezen, T. de Witte, J. Marsh, N. Kroeger on behalf of the CLWP and WPSAA. Outcome of allogeneic HSCT for patients transformed from aplastic anaemia to MDS or leukaemia. A report by the MDS subcommittee of the Chronic Leukaemia Working Party and the Aplastic Anaemia Working Party of the EBMT.

ii. E.T. Korthof, J.J.J.W. van der Zalm, D. Bresters, M.F. Raphael, J. Tolar, A. Bekassy, T. Guengoer, M. Ayas, M. Albert, F. Touzot, A. Bacigalupo, A. Fasth, M.A. Díaz, P. Veys, F. Cossu, M. Yabe, R. Oneto, C. Peters, H. Gaspar, C. Dufour, J. Marsh on behalf of the Working Parties Paediatric Diseases, Inborn Errors and Severe Aplastic Anaemia. Dyskeratosis congenita: a literature and database review of immune status and stem cell transplantation data.

iii. A. Bacigalupo. Matched sibling transplants for aplastic anaemia: survival advantage for marrow versus peripheral blood transplants in all age groups.

iv. R. Peffault de Latour, R. Porcher, C. Dufour, J. Marsh on behalf of the Severe Aplastic Anaemia Working Party (SAAWP). Outcomes and risk factors of matched donor transplantation for Fanconi anaemia: a retrospective study for the Severe Aplastic Anaemia Working Party (SAA-WP).

v. J. Marsh, G. Socié, A. Tichelli, A. Bacigalupo, A.M. Risitano, H. Schrezenmeier, P. Sedgwick, R. Oneto, A. Barrois, $\mathrm{J}$. Passweg on behalf of the EBMT Severe Aplastic Anaemia (SAA) Working Party. Prospective phase II pilot study of rabbit antithymocyte globulin (ATG, Thymoglobuline) with ciclosporin for patients with acquired aplastic anaemia and matched pair analysis with patients treated with horse ATG and ciclosporin.

vi. C. Dufour, J. Svahn, M. Pillon, R. Oneto, O. Burlando, G. Franceschetto, M. AI Jurf, J. Marsh on behalf of the SAAWP of the EBMT. Outcome of aplastic anaemia in adolescents: a retrospective analysis from the SAAWP of the EBMT.

vii. T. Brümmendorf. Telomere biology in aplastic anaemia: implications for disease pathogenesis and treatment.

EBMT Posters 2011:

i. J. Passweg, M. Wysocki, C. Flynn, G. Socié, A. Bacigalupo, R. Oneto, A. Tichelli, E.T. Korthof, H. Schrezenmeier, M. Aljurf, R.-M. Hamladji, C. Dufour, J. Marsh on behalf of the WPSAA of the EBMT. GVHD prophylaxis with or without MTX in patients receiving sibling transplants for SAA. Impact on graft failure.

\section{1}

\section{Outcome of aplastic anaemia in children. A survey on} behalf of THE SAA and PDWP of the EBMT

C. Dufour, J. Svahn, M. Pillon, R. Oneto, C. Peters, G. Franceschetto, M. Al Jurf, J. Marsh on behalf of THE SAA and PDWP of the EBMT

Aplastic Anemia's occurrence in childhood is estimated lower than in older ages. Since the data base of the SAA of the EBMT contains data on a relevant number of children with acquired $A A$ (AAA) we sought to investigate the outcome of this disease in the age between 0 and 12 years. To this end we looked into the SAAWP EBMT database and evaluated the overall survival (OS) and event free survival (EFS) in 776 patients aged 0-12 years (median fup 2.4 min 0 -max 11.3 years) in relation to the period of diagnosis (1990-1999, 2000-2009) and the received therapy (only IS, first line HSCT and HSCT after failed IS).

The 3 year probability OS and EFS were $86 \%$ and $83 \%$ respectively which reproduced those seen in period 1990-1990 (87\% OS and $85 \%$ EFS) leading to the speculation that both diagnostic and therapeutic tools, including supportive treatment, were at a level hardly susceptible of further improvement.

Focusing on post 2000, 3 year OS after first line IS therapy was $97 \%$ vs $86 \%$ for first line HSCT vs $83 \%$ for HSCT performed after having failed IS ( $p$ ns). EFS at 3 years after first line IS was $86 \%$ vs $83 \%$ for first line HSCT vs $81 \%$ for HSCT performed after having failed IS (p ns).

Since first line HSCTs included both matched sibling donor (MSD) and alternative donor transplants (ALT i.e. all other type of donors except from matched sibling donor), we compared OS and EFS of these procedures and found that MSD did significantly better than ALT (OS at 3 yrs $91 \%$ vs $78 \%$ p0.001; EFS $88 \%$ vs $76 \%$, p0.001) donor HSCT. 
When we compared the following 3 first line therapy: IS, MSD and ALT donor transplants along with HSCT done after having failed IS, we found an OS advantage for IS (97\%) over MSD HSCT $(91 \%)$ over ALT HSCT $(78 \%)$, and that OS for HSCT post IS was (83\%) ( $\mathrm{p}$ for the 4 comparisons 0.0001 ). EFS after MSD HSCT was $88 \%$, after IS wad $86 \%$ after ALT HSCT was $76 \%$ whereas HSCT post failed provided and EFS of $81 \%$ ( $p$ for the 4 comparisons 0.01 ).

Taken together these data indicate that front line IS is an excellent therapy for children with AAA, and that in case of failure of this treatment HCST is a very good salvage option. Given that HSCT is able to restore haematopoiesis more completely and more durably than IS, if a matched donor is available the preference of front line HSCT over front line IS can be justified.

\section{3}

A survey of fully haploidentical haematopoietic stem cell transplantation in patients with severe aplastic anaemia: a study by the Severe Aplastic Anaemia Working Party (SAAWP) and Paediatric Diseases Working Party (PDWP) of the European Blood and Marrow Transplantation Group (EBMT)

M.T. Lupo Stanghellini, F. Ciceri, J. Passweg, F. Locatelli, C. Peters, C. Dufour, J. Marsh, E.T. Korthof on behalf of the Severe Aplastic Anaemia Working Party (SAAWP) and Paediatric Diseases Working Party (PDWP) of the European Blood and Marrow Transplant Group

Allogeneic hematopoietic stem cell transplantation (HSCT) is one of the most important treatment options for patients (pts) with high-risk hematological diseases who have an HLA-identical sibling or matched alternative donors. In order to offer the transplant option to patients lacking a suitable matched donor, alternative options, such as unrelated cord blood transplantation (UCBT) or family haploidentical stem cell transplantation (haplo-HSCT), were developed in the last 15 years with promising results.

Information is scarce on outcomes after haplo-HSCT or UCBT for pts with severe aplastic anemia (SAA): registry surveys will better clarify the benefit of alternative transplantation in this setting. On behalf of the SAAWP and PDWP we here report the European Blood and Marrow Transplantation Group (EBMT) survey on outcomes after haplo-HSCT for pts with SAA.

We retrospectively analyzed 73 pts $(45 / 73$ male, median age at SAA diagnosis 9.78 years, at transplant 12 years, 18/73 adults) with SAA who received a haplo-SCT in 48 centers between 1976 and 2011 - 57/73 between 2000 and 2011. Thirty-one centers performed only 1 haplo-SCT, 13 centers 2 haplo-SCT, 3 centers 3 haplo-SCT, 1 center 7 haplo-SCT. Haplo-SCT was used as the first transplant option for 59 pts, as the second for 12 pts and 2 pts received haplo-SCT as third or fourth options. The median time from SAA diagnosis to haplo-SCT was 324 days (range 10-5373).

The conditioning was mostly non-myeloablative; TBI was utilized in 16 cases. Ex vivo T cell manipulation was performed in 44 cases. Neutrophil engraftment was documented in $58 \%$ of pts at a median time of +14 days (range $3-45$ ). Graft loss was seen in three pts.

Six out of 48 pts with documented engraftment experienced acute GvHD grade I, 5 grade II, 4 grade III 4 grade IV and 1 with unknown grade.

Twenty-nine pts are alive (median follow-up 1015 days, range 0-6589) - 6/29 received transplant before 2000, 23/29 after 2000. Median time to exitus was 93 days (range 4-2710) - main causes of death being infections (24/44), GvHD (6/44), hemorrhage (6/44).

At a median follow-up of 33 months the estimated probability of 3 -year overall survival (OS) was $37 \%$. There was a trend for improved OS for pts transplanted in centrs who performed at least 3 haplo-HSCT compared to other sites $(58 \%$ versus $30 \%, p=0.0578)$. No statistically difference was documented for transplants performed before and after 1999, year of widespread diffusion of cell-selection devices.
To our knowledge, our survey provides information on haploHSCT for pts with SAA for the first time. Development of new strategies on haplo-HSCT will further improve outcome results in this setting.

164

Characteristics and outcome of severe aplastic anaemia in HIV positive patients

R. Peffault de LaTour, J. Marsh (Paris, FR; London, UK)

The care of the human immunodeficiency virus (HIV)-infected patients has changed dramatically since the widespread use of combined antiretroviral therapy (CART) in 1996. Current cART regimens suppress viral replication, provide significant immune reconstitution, and have resulted in a substantial and dramatic decrease in acquired immunodeficiency syndrome (AIDS)related complications and deaths in both adults and children. Those patients are now experiencing long-term follow-up and notably auto immune diseases have been described in this context. Severe Aplastic Anemia (SAA) is an immune-mediated disorder in most cases with active destruction of hematopoietic cells by effector T lymphocytes. The association of HIV-infected patients and SAA is exceptional. There are only very few documented cases of allogeneic stem cell transplantation (SCT) or immunosuppressive therapy in HIV patients with acquired SAA. The aim of this study is to collect all cases of HIV-infected patients who developed an acquired SAA to better characterize this entity and eventually its specific treatment.

\section{Working Party Session Chronic Leukaemia}

\section{2}

Retrospective comparison of reduced-intensity conditioning and conventional myeloablative conditioning for allogeneic haematopoietic stem cell transplantation using HLA identical sibling donors in AML and MDS with less than $10 \%$ bone marrow blasts

R. Martino, A. Van Biezen, L. de Wreede, M. Fiocco, P. von dem Borne, M. Robin, L. Volin, M. Bornhäuser, T. De Witte, V. Rocha, $N$. Kröger, M. Mohty, for the ALWP and the subcommittee for MDS of the CLWP of the EBMT

In this multicenter retrospective study, the long-term outcomes of 878 adults with acute myeloid leukemia (AML) and refractory anemia with excess blasts (RAEB) in an early/intermediate status (bone marrow blasts $<10 \%$ ) who underwent transplantation with a human leukocyte antigen (HLA)-identical sibling donor between 1998 and 2004 were analyzed according to 4 regimens of conditioning intensity: reduced-intensity conditioning (RIC) in 160 patients (divided into intermediately RIC [Interm$\mathrm{RIC}]$ and non-myeloablative [NMA] RIC), and myeloablative conditioning (MC) in 718 patients (divided into conventional MC [ConvMC] and hyperintense MC [HyperMC]). In multivariate Cox analysis, the 7-year nonrelapse mortality incidence (NRM) was influenced by the conditioning regimen in a time-varying manner: up to day +100 , the cause-specific hazard for NRM was significantly lower for the RIC subgroups (hazard ratio [HR], 4.3; 95\% confidence interval [95\% Cl], 4.3-11.1; $\mathrm{P}<0.01$ ); but a land-mark analysis beyond day +100 showed that the 7-year cause-specific hazard for NRM was increased after NMA conditioning (HR, 2.4; 95\% Cl, 1.2-10.4; $\mathrm{P}=0.04)$. The 7 -year relapse was also influenced by the conditioning regimen in a time-varying manner: up to month +12 , the relapse incidence was significantly higher after NMA conditioning (HR, 2.4; $95 \% \mathrm{Cl}, 1.2-10.4 ; \mathrm{P}=0.04$ ); but a land-mark analysis beyond +12 months showed that the 7 -year relapse incidence was not influenced by the conditioning intensity. The 7 -year probabili- 
ties of progression-free and overall survival were significantly lower only in the NMA subgroup (Multivariate $\mathrm{P}<0.01$; and 0.01 , respectively). In conclusion, the similar 7-year survival after IntermRIC and SMC is encouraging, since IntermRIC patients were older (age $>50$ years in $75 \%$ vs. $15 \%$, respectively, $\mathrm{P}<0.001)$ and had higher EBMT risk scores pretransplantation. However, 7-year outcomes were worse in NMA recipients. Our data suggest that prospective studies comparing RIC regimens (especially IntermRIC) with ConvMC are appropriate in patients with AML and RAEB who are in a non-advanced disease status and are eligible for either convMC or RIC regimens.

\section{3}

Effect of prior therapy with nilotinib or dasatinib on the outcome after allogeneic stem cell transplantation for patients with chronic myeloid leukaemia; a non-interventional prospective study by the CML subcommittee of the chronic leukemia Working Party M. Schleuning, A. van Biezen, J. Hoek, J. Apperley, M. Aljurf, L. Volin, M. Markiewicz, B. Afanasyev, J. Finke, M. Michallet, P. Browne, A. Nagler, T. de Witte, E. Olavarria on behalf of the CML subcommittee of the chronic leukemia Working Party of the EBMT

Introduction: Stem cell transplantation will continue to be a treatment option for patients with chronic myeloid leukaemia, despite the introduction of tyrosine kinase inhibitors. However, many patients will have received prior therapy with TKIs, including Nilotinib or Dasatinib at the time of allogeneic stem cell transplantation.

While the use of Imatinib prior to stem cell transplantation seems to have no adverse impact on the outcome of allogeneic stem cell transplantation little is known on the impact of prior use of second generation TK inhibitors.

Objective: To evaluate the influence of prior treatment with second generation tyrosine-kinase inhibitors (TKIs) in CML patients on engraftment rates and treatment-related mortality during and after treatment with allo-HSCT. Secondary objectives: to evaluate the effect of prior 2 nd generation TKIs on treatment-related toxicity after treatment with allo-HSCT in CML (i.e. incidence and severity of acute and chronic GVHD, SOS (VOD) of the liver, pulmonary complications, and causes of treatmentrelated mortality); to evaluate overall and disease-free survival; and to evaluate the relapse rate.

Methodology: All transplant-related data will be collected prospectively, whereas data on prior TKI therapies are recorded retrospectively. The study started at the beginning of 2010 . A total of 450 patients are envisaged for analysis. Today, 68 centres have registered for participation. Together they expect to be able to submit 475 patients for this study, of which 163 patients have been included so far. Transplant dates range from November 2009 to December 2011. For two patients, the allotransplant was not the first transplant.

Preliminary results: Data entry has been done for 128 patients. In a very preliminary analysis of those 128 patients, 4 patients showed a graft failure. $2 / 3$ of the patients were transplanted in $\mathrm{CP}$ and $73 \%$ were transplanted beyond $12 \mathrm{mo}$ after diagnosis. The median time from diagnosis to transplant was 21 months and the median age at transplant was 47 years (18-67y). Sixtyseven percent of pts were male and $33 \%$ female. The majority $(55 \%)$ received transplants from matched unrelated volunteer donors. At the CLWP session in Geneva all data received by that date will be presented.

Centres that are interested in participating in this study can contact the EBMT Data Office in Leiden via clwpebmt@lumc.nl.

\section{5}

Data quality initiative in CLL

J. Schetelig (1), A. van Biezen (2), N. Smedegard Andersen (3), M. Karas (4), M. Machaczka (5), C. Moreno (6), S. Paneesha (7), P. Dreger (8), T. de Witte (9), M. van Gelder (10)

(1) Dresden, DE); (2) Leiden, NL; (3) Copenhagen, DK; (4) Pilzen, CZ; (5) Stockholm, SE; (6)(Barcelona, ES; (7) Birmingham, UK; (8) Heidelberg, DE; (9)(Nijmegen, NL; (10) (Maastricht, NL

Data quality is a general concern in the context of clinical studies. With respect to CLL a double challenge exists: First, current data requested in the disease classification sheet in minimum essential data $A$ (MED $A$ ) are insufficient to describe the indication for allogeneic HCT. This is because information on cytogenetics and pre-treatment (especially purine-analogue refractoriness) has not been defined as minimal essential data in past times. MED B data is only sparse in the database. Second, missing data hinder the analysis of important clinical aspects. This double challenge is addressed in three ways:

1) Prospective non-interventional studies shall guarantee better future data quality through limited MED B information and active data request in small disease entities like 17p-CLL, T-PLL or Richterss syndrome.

2) A data quality initiative based on a reduced MED B form will be launched for first allogeneic transplants dating back until 2000.

3) The disease classification sheet in MED A CLL will be redefined together with CIBMTR and the amount of information requested in MED B will be reduced.

\section{6}

Haematopoietic stem cell transplantation in recipients of solid organ transplants: a restrospective survey by the Complications Subcommittee of the Chronic Leukaemia Working Party of the EBMT

G.W. Basak, S. van der Werf, W. Wiktor-Jedrzejczak, T. de Witte, T. Ruutu on behalf of the Chronic Leukemia Working Party, EBMT

Hematopoietic stem cell transplantation (HSCT) is a constantly evolving procedure. Due to many improvements its safety is increasing, which translates into lower mortality rates and expansion of new indications. A number of novel approaches such as reduced intensity or non-myeloablative conditioning regimens have made HSCT feasible even in elderly patients with comorbidities. Therefore some patients who would not have been considered as candidates for HSCT before undergo this procedure nowadays. The number of surviving patients who have undergone a solid organ transplantation (SOT) is growing. Similar to healthy people, they may also suffer from a number of hematopoietic disorders which are indications for HSCT. Moreover, the probability of developing such disorders may be increased due to immunosuppression. Despite the increased risk associated with a HSCT procedure in these patients, apparently a significant number of patients have undergone autoSCT or alloSCT following SOT in Europe. We decided to perform a retrospective study within the Complications Subcommittee of the Chronic Leukemia Working Party in order to document the outcome of HSCT in these patients and to investigate how the HSCT procedure has affected the functions of transplanted organ. All patients of all ages who have undergone alloSCT or autoSCT following SOT can be included in this study. The centers participating in this study are asked to fill in the MED-A form and a shortened MED-B form for the HSCT (prefilled based on already reported data). In addition, a specific MED-C form of the SOT (separate forms for heart, kidney, liver and other organ transplants) has to be filled in. The centers in which recipients of solid organ transplant have ever undergone either auto- or alloSCT are encouraged to include their patients. Centres that are interested in participation should contact the Leiden Data Office via clwpebmt@lumc.nl. 
217

A non-interventional prospective study to evaluate the effect of transfusions and iron toxicity in patients with myelodysplastic syndromes treated with allogeneic stem cell transplantation

E. Cremers, A. van Biezen, L. de Wreede, M. Scholten, J. Finke, L. Volin, D. Beelen, Y. Fløisand, T. de Witte, N. Kröger a study on behalf of the MDS subcommittee of the Chronic Leukaemia Working Party of the EBMT

Objectives: To prospectively evaluate the effect of iron toxicity on treatment outcome after myeloablative (MAC) and reduced intensity (RIC) allogeneic SCT, in previously untreated adults with high risk MDS. This report describes correlations between pre-transplant parameters at time of SCT and 100 days after SCT.

Methods: The data collection by the CLWP of the EBMT, started in March 2010. This survey collected prospectively data on diagnosis, SCT, iron parameters, transfusions, comorbidity and treatment outcome. A composite iron score based on serum iron levels, transferrin saturation and serum ferritin levels is used to define a high risk group with serum ferritin $>1,500 \mathrm{ng} / \mathrm{ml}$, and/or transferrin saturation $>80 \%$ and/or serum iron $>200 \mathrm{mg} / \mathrm{l}$.

Results: From the 134 patients registered so far, additional data is available on 94 patients. Eighty-three patients had MDS and 9 patients MPS, of whom 59 were male. WHO-classifications at time of SCT are 7 patients with RA/RARS, 8 with RCMD, 21 with RAEB-1, 28 with RAEB-2 and 1 with sAML. Forty-one patients received a MAC and 53 a RIC regime. After conditioning 31 patients received stem cells from a sibling, 2 from a mismatched relative and 61 patients from an unrelated donor. Time between diagnosis and SCT was $<6$ months for 36 patients and $>6$ months for 58 patients. In 41 patients GvHD occurred (grade I-IV). Variables analyzed in 69 patients (day 100 data available) were iron load at day of SCT and +100 days, amount of red blood cell transfusions prior to SCT and between SCT and +100 days and occurrence of GvHD.

We observed a strong positive correlation between ferritin levels pre-SCT and +100 days $(r=0.64 ; p=0.001)$. Similarly, a positive correlation exists between the iron score pre-SCT and +100 days $(p=0.006$; $O R=18.00)$. Despite the preliminary nature of this analysis there was a strong indication that the increased transfusion burden prior to SCT is positively correlated with the occurrence of aGvHD ( $p=0.039$; OR=4.13). So far, we found no correlation between transfusion burden pre-SCT and +100 days (Spearman $r=0.004 ; p=0.98$ ) and no correlation between transfusion burden and iron score at time of SCT $(p=0.226$; $\mathrm{OR}=2.30$ ). The relation between mortality before day 100 and transfusion load was not significant $(p=0.145$; $O R=3.73)$.

Conclusion: this prospective observational study is running well. Preliminary data indicate that we may expect sufficient data to achieve the primary objectives of this study.

\section{9}

Recommendations for standard GVHD prophylaxis and treatment: an EBMT/ELN consensus project

T. Ruutu, D. Niederwieser, A. Gratwohl, T. de Witte on behalf of an EBMT/European LeukemiaNet (ELN) consensus working group

Graft versus host disease (GVHD) remains one of the main obstacles to broader application of alloge-neic haematopoietic stem cell transplantation (HSCT). It can be prevented with selected methods, but often at the expense of an increased risk of relapse and rejection. To advance our knowledge and de-fine optimal approaches, correct information on the value of the different strategies and methods in GVHD prevention and treatment is essential. A recent survey of the EBMT documented once again that GVHD prophylaxis and treatment strategies and techniques used in allogeneic stem cell transplantation are very heterogenous. Even in superficially similar protocols, the details in practical work differ con-siderably. There are no widely accepted standard practices for the prevention and management of GVHD. Centre effects have been demonstrated in transplantation outcomes. They are attributed to centre size, macroeconomic aspects and JACIE accreditation status. Differences in outcome might as well be, at least partly, due to differences in treatment strategies and transplantation techniques. A prerequisite to learn about this is to have a common comparator. For this purpose a current "standard" practice needs to be defined. These guidelines could lead towards a more uniform practice and they could be used as baseline when evaluating the impact of different components of the transplant proc-ess. Based on available literature and current practice, a joint consensus committee of the EBMT and ELN is working on a proposal for the implementation of minimal EBMT/ELN standards in this field.

\section{Working Party Session Paediatric Diseases}

220

Identification of antileukaemic NK cell subsets using the CD107a mobilisation assay

P. Schlegel, P. Lang, K. Witte, R. Teltschik, R. Handgretinger, M. Pfeiffer

University Children's Hospital (Tübingen, DE)

Identification of NK cells with high antileukemic activity will help to improve targeted cellular therapy strategies, especially in the context of haploidentical stem cell transplantation. We screened the NK cells of mismatched healthy donors with regards to their reactivity against ALL blasts to identify such NK cell subsets.

Method: NK cells were isolated from 4 healthy donors with CD56+ MACS beads and incubated with 5 different primary pediatric ALL blasts in a 3 hour CD107a assay. Analysis of the NK cells considered the main KIR receptors CD158a, CD158b and CD158e, as well as ILT2, NKG2a and activating receptors NKG2D, NKp30, NKp44 and NKp46. Blasts were analyzed for NKG2D ligands MIC A and B, ULBP1-3 and adhesion molecules CD11a, CD50, CD54, CD58, CD112 and CD155. Degranulation controls were performed with $\mathrm{K} 562$ as target cells.

Results: Degranulation of the whole NK cell compartment displayed a wide variability (range of CD107a+ cells: $0.6-11.3 \%$ ) and was influenced by type of donor and type of ALL blasts and was not predictable according to common alloreactivity models in this data set. A significant increase $(p<0.001, n=20)$ of the CD107a+ fraction was reached by overnight IL2 stimulation of NK cells. In contrast to the entire NK cell compartment, the subset of KIR- NK cells revealed a high and stable indirect cytotoxicity in terms of CD107a expression throughout all experiments, whereas the KIR+ NK cell subset by trend followed the receptor-ligand model. ALL blasts showed no or low expression of NKG2D ligands: MIC A $(0-2.2 \%)$, B $(0-1.6 \%)$, ULBP1$3(0-1.6 \%)$. Adhesion molecules were diversely expressed on the blasts: CD50 (65-99\%), CD54 (48-77\%), CD58 (12-98\%), CD112 (12-63\%), CD11a (2.8-34\%), CD155 (0-2.2\%). There was no correlation of NK cell degranulation with expression of adhesion molecules or NKG2D ligands on the blasts.

Conclusion: Our aim was to identify NK cell subsets with high activity against pediatric ALL blasts for potential use in cellular therapies. The KIR- NK cell subset was found to show high and stable degranulation disposition, independent from the target and from the NK cell donor. Moreover donors displayed an individual non-predictable variability in the level of overall NK cell degranulation. This suggests including functional analyses of NK cells in donor selection strategies. Further investigations are necessary to confirm these data. 


\section{1}

Incidence and mortality of adenovirus infection after paediatric allogeneic stem cell transplantation - A comparison between bone marrow and CD3/19 depleted peripheral blood stem cell transplants

A.M. Willasch, J. Soerensen, A. Jarisch, A. Berger, H. Rabenau, C. Brandt, E. Rettinger, G. Lucchini, L. Budig, G. Sach, U. Koehl, H. Kreyenberg, T. Klingebiel, P. Bader

Goethe University Frankfurt (Frankfurt, DE)

Infection with human adenoviruses (ADV) can cause lifethreatening infections in pts after allo-SCT and represents a major reason for transplant related mortality (TRM, in historical cohorts after haplo-SCT up to $30 \%$ ). Graft manipulation by T-cell depletion and the use of immunosuppressives affect immune reconstitution and can extend duration of impairment of immunity after SCT. Delayed reconstitution of immunity increases the risk for viral infections. New preparative regimens such as reduced intensity conditioning (RIC) followed by CD3/19 depleted PBSC transplant (3/19depl-PBSCT) try to overcome these limitations

To substantiate this we studied the incidence (inc.) and mortality of ADV infection after ped. allo-SCT overall and as a function of graft. 210 transplants have been performed in 200 ped. pts in Frankfurt between ' 05 and '11. Donor source was 3/19depl-PBSC $(n=95)$ and BM $(n=115)$. Median follow up was 13.9 mths. Weekly post-transpl. ADV-screening was conducted by qPCR in plasma, throats swabs and faeces. Inc. of ADV detection in any compartment at any time-point was $40.0 \%$ and sign. higher after $3 / 19 \mathrm{depl-PBSCT}$ compared to BMT; $49.5 \%$ vs $32.2 \%$ ( $=0.016$ ). Inc. was $26.7 \% \leq d 60,20.6 \% d 61$ to 100 and $14.0 \%>\mathrm{d} 100$

Cumulative inc. of $3 y$ TRM ( $\mathrm{Cl}$ 3y TRM) due to ADV disease was $4.6 \%$ (3/19depl-PBSCT $6.9 \%$, BMT $3.0 \%$; n. sign.). In detail, $14.5 \%$ of pts $(29 / 200)$ died due to non relapse mortality. Amongst these 29 pts, 16 (55.2\%) were ADV pos.. ADV positivity was sign. more frequent after $3 / 19$ depl-PBSCT compared to BMT $(84.6 \%$ vs $31.3 \%$; $p=0.008)$. Mortality due to ADV disease among TRM pts was $27.6 \%$ (3/19depl-PBSCT $38.5 \%$, BMT $18.8 \%$; n. sign.). Overall $\mathrm{Cl} 3 \mathrm{y}$ TRM was sign. higher in ADV pos. $(n=84)$ vs neg. $(n=126)$ pts $(28.7 \%$ vs $13.4 \%, p=0.03)$. Taken together, ADV was detected in every second pt after 3/19depl-PBSCT. Although the CI 3y TRM due to ADV disease was low (4.6\%), post-transpl. ADV detection sign. increased the risk for subsequent TRM.

In summary, ADV represents a severe threat to survival, particularly in pts after 3/19depl-PBSCT. Apparently, more rapid reconstitution of immunity in these pts compared to historical mega-dose CD34 transplants resulted in low mortality due to ADV disease in this high-risk group. Nevertheless, consequent post-transpl. screening for ADV is indispensable. Early and effective treatment eg with Cidofovir or DLI (ADV-specific donor T-cells, cytokine-induced killer cells $(\mathrm{CIK})$ ) is important for further reduction of ADV-related mortality.

\section{2}

Meta-analysis on treosulfan for conditioning in children and adolescents before haematopoietic stem cell transplantation

U. Poetschger, K.-W. Sykora, M. Zecca, P. Veys, A. Lankaster, A. Cant, J. Wachowiak, S. Lyon-Caen, C. Peters on behalf of the EBMT Paediatric Diseases Working Party

A retrospective analysis was performed in children and adolescents who received treosulfan for conditioning before allogeneic or autologous HSCT to identify possible dose related toxicity and determine the incidence of engraftment, treatment related mortality (TRM), overall survival (OS) and event free survival (EFS). Patients below 18 years registered in the EBMT database who underwent HSCT between January 2005 and July 2010 for malignant or non malignant disease were eligible. 843 patients met the inclusion criteria and $75 \%$ could be included into the analysis (521 allogeneic, 83 autologous). In the group of patients with allogeneic HSCT, 41 were below the age of 6 months and 65 between 6 -months and 1 year. 314 were between 1 and 12 years of age and 101 above 12 years. 165 patients suffered from malignancies, 356 patients were transplanted for non malignant diseases. 437 underwent a first HSCT, 87 had a following HSCT. For allogeneic HSCT, the median treosulfan dose was $42 \mathrm{~g} / \mathrm{m}^{2}(12-46) .24$ patients $(5 \%)$ received less then $33 \mathrm{~g} / \mathrm{m}^{2} .153$ patients $(29 \%)$ received between 33 and $39 \mathrm{~g} / \mathrm{m}^{2}$. The majority of patients received a treosulfan dose between 39 and $45 \mathrm{mg} / \mathrm{m}^{2}$ (332 patients, $62 \%$ ). 13 patients $(2 \%)$ received more than $45 \mathrm{mg} / \mathrm{m}^{2}$.

Results: The most common grade 3 or 4 toxicities were diarrhoea $(24 \%)$, stomatitis $(22 \%)$, and SGOT $(25 \%)$. We did not find any positive correlation between the given dose and the grade 3 or 4 toxicity in the dose range used within the EBMT centres. Acute graft-versus-host disease (GvHD) of grade 3 or 4 occurred in $10 \%$ of the patients and the rate of limited and extended chronic GvHD was $13 \%$ and $6 \%$, respectively. OS and EFS correlated significantly with diagnoses: 3-year OS was $51 \%$ for ALL, $46 \%$ for AML, $80 \%$ for inherited disorders, and $93 \%$ for haemoglobinopathies. OS was significantly higher in patients who received treosulfan for first HSCT. 3-year EFS was $39 \%$ for $A L L$ and $40 \%$ for AML. In the given dose range, we did not find an association between dose and GvHD, OS, EFS, relapse incidence and TRM

Conclusion: We identified no dose related toxicity, graft failure, GVHD, TRM, or relapse incidence in malignant diseases. These results emphasise the low toxicity profile of treosulfan, even in heavily pre-treated children and adolescents and in patients undergoing a second HSCT.

\section{3}

Allogeneic stem cell transplantation using busulfan, cyclophosphamide, and melphalan for children with acute myeloid leukaemia in second complete remission: retrospective interphase analysis on behalf of the German AML-BFM study data from the paediatric BFM

R. Beier (1), M.H. Albert (2), P. Bader (3), A. Borkhardt (4), U. Creutzig (1), M. Eyrich (5), B. Gruhn (6), J. Greil (7), R. Handgretinger (8), W. Holter (9), B. Kremens (10), P. Lang (8), I. Müller (11), C. Mauz-Körholz (12), R. Meisel (4), C. Peters (13), R. Reinhardt (1), C. Rössig (14), P. Sedlacek (15), A. Schulz (16), F. Schuster (4), A. Schrauder (17), B. Strahm (18), K.W. Sykoa (1), W. Wössmann (19), M. Zimmermann (1), M.G. Sauer (1)

(1)Medizinische Hochschule (Hannover, DE); (2)von Haunersches Kinderspital (Munich, DE); (3)Universitätskinderklinik (Frankfurt, $D E) ;(4) U n i v e r s i t a ̈ t s k i n d e r k l i n i k$ (Düsseldorf, DE); (5)Universitätskinderklinik (Würzburg, DE); (6)Universitätskinderklinik (Jena, DE); (7)Universitätskinderklinik (Heidelberg, DE); (8)Universitätskinderklinik (Tübingen, DE); (9)Universitätskinderklinik (Erlangen, DE); (10)Universitätskinderklinik (Essen, DE); (11)Universitätskinderklinik (Hamburg, DE); (12)Universitätskinderklinik (Halle, DE); (13)St Anna Kinderspital (Vienna, $D E) ;(14)$ Universitätskinderklinik (Münster, DE); (15)University Childrens Hospital (Prague, CZ); (16)Universitätskinderklinik (UIm, DE); (17)Universitätskinderklinik (Kiel, DE); (18)Universitätskinderklinik (Freiburg, DE); (19)Medizinische Universitätskinderklinik (Gießen, DE)

Data from the pediatric BFM 98 trial show that the majority of children with AML do not benefit from allogeneic hematopoietic cell transplantation (HCT) in first remission. Therefore, HCT was only recommended for patients achieving a second remission after first relapse. Assuming a dismal prognosis, the potentially toxic triple alkylator regimen consisting of busulfan, cyclophosphamide, and melphalan (BuCyMel) was recommended, based on the practice of the EWOG-MDS 98 trial and the AIEOP AML 2002/02 study. Here we retrospectively analyze the interim data of children who underwent HCT after BuCyMel for AML in CR2 between 1998 and 2009 before the 
initiation of a prospective, controlled and centrally monitored AML-BFM HCT trial.

Out of 152 identified children transplanted in 17 German pediatric transplant centers after achieving CR2, complete data were available for 62 children after a preparative regimen with BuCy$\mathrm{Mel}$ and for 54 receiving a different regimen. For the first group 62 children were identified for analysis. The median age at HCT was 11.9 years [3.0; 18.3]. GVHD-prophylaxis mostly consisted of CSA and short term MTX with or without ATG. Matched sibling donors were used for 23 of 116 analyzed recipients, the remaining received grafts from matched or partially mismatched unrelated donors. The overall survival at 5 years (5y OS) for children achieving CR2 was $61 \%$ (standard error $0.07 \%$ ). Relapse mortality was $21 \%$ (13/62 children). Treatment related mortality reached $16 \%(10 / 62)$ and was caused by infection (4/10), GvHD (4/10) and multi organ failure of unknown cause $(2 / 10)$. Within the same observational period 54 children received a HCT in CR2 using a regimen other than BuCyMel. 5y OS in this group was $35 \%(19 / 54$ children, standard error $0.06 \%)$ with a TRM of $29 \%(16 / 62)$ and mortality from second relapse reaching $30 \%(17 / 62)$.

Conclusion: Even taking into account possible selection bias in this retrospective analysis, HCT in CR2 using ByCyMel resulted in a respectable overall survival of $61 \%$. TRM in this cohort was higher than the one reported by the AIEOP group for children in CR1 and seems to be lower than in EWOG-MDS 98. The prospective, controlled and centrally monitored trial AML SCT-BFM 2007 has started to recruit patients in January 2010. This trial aims to generate solid and valid outcome data for children with AML in CR2 or primary refractory disease to serve as a platform for the development of further transplant strategies.

\section{4}

EBMT Survey on adolescents with ALL in 2nd CR given allogeneic HSCT. Analysis on behalf or the ALWP and PDsWPs

G. Dini, M. Labopin, M. Ayas, J. Cornish, M. Potter, F. Locatelli, M. Zecca, Y. Bertrand, G. Socié, R. Hough, A. Balduzzi, S. Lawson, E. Lanino, S. Giardino, M. Mohty, V. Rocha, C. Peters on behalf of the ALWP and PDSWPS

Adolescents are treated both by paediatric teams (group 1, defined as centres reporting a majority of pts $<18 \mathrm{yrs}$, or combined centres, with a dedicated paediatric team) and by adult teams (group 2, defined as centres reporting pts below and above 18 yrs, without a dedicated paediatric team). The primary end-point of this study was to evaluate the impact of the type of centre, i.e., paediatric or adult, on outcome after HSCT from a MSD, or from a volunteer UD in adolescents with ALL in $2 n d$ CR, defined as pts older than $14 \mathrm{yrs}$ and younger than $18 \mathrm{yrs}$ at HSCT, given allogeneic HSCT from 1995 to 2009 and reported to the EBMT registry. A total of 435 pts $(67 \%$ males and $33 \%$ females in group $1,70 \%$ males and $30 \%$ females in group $2, p=n s$ ) were included in the study; 214 pts were transplanted in paediatric centres, whereas 221 pts were transplanted in adult centres. The 2 groups differed in terms of distribution of patient and transplant-related variables: the frequency of very early relapse in group 2 pts was approximately twice as high as in group $1(p=0,001)$; the median interval from diagnosis to transplant was shorter in group $2(p=0.003)$, the median age at diagnosis was higher for group 2, $(p=0.0001)$. A MSD was used in $40 \%$ of the pts in group 1 and in $61 \%$ of those in group 2; the remaining pts received an UD HSCT $(p<0.001)$; the source of stem cells was bone marrow in $77 \%$ of pts in group 1 and in $58 \%$ of pts in group 2; the remaining pts received PBSCs $(p<0,0001)$. The transplant conditioning regimen included TBI in $86 \%$ and $81 \%$ of group 1 and 2, respectively $p=n s$ ). As of September 2011,the median follow-up was 49 months. $40 \%$ of the pts from group 1 and $42 \%$ of the pts in group 2 reported acute GVHD grade $2-4$, respectively $(p=n s)$. The 2 year cumulative incidence of chronic GvHD was $24 \%$ for group 1 and $32 \%$ for group 2, respectively $(p=n s)$.
Five-year Kaplan-Meyer estimates of LFS were $43 \%$ for group 1 , versus $31 \%$ for group 2 , respectively $(p=0.02)$. The 5 year cumulative incidence of TRM and relapse rate were $31 \%$ and $26 \%$ for group $1,26 \%$ and $43 \%$ for group 2 ( $p=N S$ and $p=0,0006)$. Univariate analysis showed that year of HSCT $<2000(p=0,01)$, early relapse $(P=0.0004)$ and diagnosis to transplant interval $<31$ mos $(p<0.0001)$ were poor-risk factors for LFS. Multivariate analysis confirmed for adolescents treated at paediatric centres a significantly lower relapse rate and a trend towards better LFS, while no difference was observed in chronic GvHD.

\section{5}

A simplified method for busulfan therapeutic drug monitoring using dried blood spot sampling in paediatric patients undergoing stem cell transplantation

C.R.S. Uppugunduri (1), Y. Théorêt (2), J. Déglon (3), F. Versace (3), F. Gumy-Pause (1), H. Ozsahin (1), P. Dayer (1), J. Desmeules (1), Y. Daali (1), M. Ansari (1)

(1)Geneva University Hospitals (Geneva, CH); (2)University of Montreal (Montreal, CA); (3)University of Geneva (Geneva, $\mathrm{CH}$ )

Background: Busulfan (Bu) is one of the commonly used agents for conditioning of pediatric patients prior to hematopoietic stem cell transplantation (HSCT). Intravenously administered $\mathrm{Bu}$ in children exhibits small therapeutic window requiring therapeutic drug monitoring. The dosage of Bu is adjusted based on the first dose pharmacokinetic parameters. Dried blood sampling may facilitate processing of the samples with less turn around times. Objective: To evaluate the utility of dried blood sampling (DBS) and dried plasma sampling (DPS) using only 5 micro liters of biofluid for therapeutic monitoring of Bu levels.

Methods: blood samples were collected from two children following the infusion of Bu after doses 1,2,3,5 and $9(n=34)$. Then, 5 micro liters each of whole blood and plasma were spotted onto Whatman 903 DBS cards and dried at room temperature for about 30 minutes. The entire spots were cut and then analyzed by a validated LC-MS/MS method. Bu was also measured by established gas chromatography coupled to mass spectrometry (GC-MS) using plasma $(n=13)$ to compare both methods. Results: A good correlation was observed between the $\mathrm{Bu}$ levels measured by DBS and DPS $\left(r^{2}=0.95\right.$; slope $\left.=0.84\right)$. The Bu levels measured by DPS $\left(r^{2}=0.92\right.$; slope $\left.=0.95\right)$ and DBS $\left(r^{2}=0.91\right.$; slope $\left.=0.80\right)$ were correlated with those measured by standard GC-MS method. The levels estimated by DBS were less than those obtained by DPS and GC-MS methods. The hematocrit (Hct) values of two children were in the range of 25.6 to $30.3 \%$, suggesting the influence of $\mathrm{Hct}$ on the measured $\mathrm{Bu}$ levels by DBS sampling. Therefore, Hct value of the patient must be considered when whole blood sample is used to estimate the plasma levels of $\mathrm{Bu}$. The plasma levels of Bu estimated using the formula "Plasma levels (analyte) = DBS levels analyte/ (1-hematocrit)" were higher than those obtained by DPS or GC-MS suggesting that a fraction of Bu binds to blood cells.

Conclusion: This method can be applied with only 5 micro liters of whole blood for routine therapeutic drug monitoring of busulfan with accuracy and faster turnaround times. The use of capillary whole blood collected after a small finger prick is under evaluation for its feasibility and accuracy to monitor busulfan levels in pediatric patients.

\section{6}

Treatment of childhood leukaemia with haploidentical haematopoietic stem cell transplantation using parent as donor: a single-centre study of 100 cases

Y. Sun, T. Wu, R. Sun, Z. Wei, J. Wang, X. Cao, Y. Lu, Y. Zhao, J. Zhou, Y. Gao, Y. Yin, S. Ji, Y. Zhang, W. Da, D. Lu

Beijing Daopei Hospital (Beijing, CN)

Our previous studies have demonstrated that haploidentical hematopoietic stem cell transplantation (haplo-HSCT) has 
achieved comparable outcomes with identical sibling transplant (Lu DP et al., Blood 2006; 107:3065), and the results of haploHSCT are similar overall between adults and children (Dong L et al., Biol Blood Marrow Transplant 2011;17:1205).

Objectives: In present study, the outcomes of childhood leukemia treated with haplo-HSCT using parent as donor were evaluated and the risk factors for survival were identified. Methods: One hundred consecutive cases from June 2002 to June 2011 in our center were retrospectively analyzed. The median age of patients was $10(3-14)$ years old. The diagnosis included AML (43\%), ALL (42\%), CML (10\%), and mixed lineage leukemia $(5 \%)$. Transplants in early, intermediate, and advanced disease were $47 \%, 32 \%$ and $21 \%$. HLA mismatched at $1,2,3$ loci was $7 \%, 22 \%, 71 \%$, respectively. Transplants in father to son, father to daughter, mother to son, and mother to daughter were $40 \%, 7 \%, 27 \%, 26 \%$ respectively. Major clinical characteristics among these four arms were similar. All patients received unmanipulated combined marrow and peripheral blood stem cells for transplant after conditioning with busulfan and cyclophosphamide (Cy)/Cy and total body irradiation plus antithymocyte globulin.

Results: Durable hematopoietic reconstitution was seen in $98 \%$ of recipients. Engraftment failure occurred in 2 cases of father to daughter transplants. One-hundred-day transplant-related mortality (TRM) was only $4 \%$. The cumulative incidences of grade II to IV acute graft-versus-host disease (aGvHD) and chronic GvHD (cGvHD) were $33 \%$ and $22 \%$, respectively. With the median follow-up of 32 (3-108) months, one-year and 5year overall survival (OS) rates for all patients were $75.9 \%$ and $68.5 \%$. Five-year OS rates for patients in early, intermediate and advanced disease were $79.5 \%, 72.0 \%$ and $52.6 \%$ $(p=0.022)$. Five-year OS of transplants in father to son, father to daughter, mother to son ,and mother to daughter were $81.5 \%$, $36.2 \%, 63.4 \%, 66.8 \%$, respectively $(p=0.028)$.

Conclusions: Under current protocol, children with leukemia tolerate haplo-HSCT from their parent very well with lower TRM, less CGVHD, and better OS compared with our published data. Disease status pre-transplant and donor-recipient relationship have significant impact on survival.

\section{Statistical Symposium}

\section{1}

Cure models: are they telling the same story as Cox regression?

U. Pötschger (1), H. Heinzl (2), M. Mittlböck (2)

(1)Children's Cancer Research Institute (Vienna, AT); (2)Center for Medical Statistics, Informatics, and Intelligent Systems (Vienna, AT)

For the statistical symposium: There has been a recurring interest in modelling survival data in the presence of 'cured' individuals. 'Cure' is defined on a population level and means that there is no longer excess mortality compared to the general population. In a paediatric population the baseline mortality may be very close to zero. Here, given sufficient follow-up, survival curves that reach a stable plateau indicate the presence of long-term survivors or cured individuals. This is quite common in paediatric oncology, where Cure-models have been developed to analyse failure time data with long-term survivors. In a more general setting, a particular type of Cure-model that incorporates the expected background mortality can be used. The aim of this work is to compare several types of Cure-model and contrast them to standard survival models. Their use, values, and limitations will be illustrated on data in the field of paediatric stem-cell transplantation.

Standard methods of survival analysis, like Cox-regression or the log-rank test, rely on the proportional hazards assumption. The results from Cox and Cure models are very similar when the hazard ratio is constant over time. However, with Cox-regression, it is not possible to distinguish between 1) patients who are cured and are long-term survivors and 2) patients who will die from their disease although a prolongation of survival can be achieved. With non-proportional hazards, the results from Coxmodels may be misleading and have no clear interpretation. In non-proportional hazard situations, Cox and Cure-models give different results. One can find examples on non-proportional hazards in the comparison of treatments with different intensities. One treatment may produce more early toxic events but is more efficient in preventing disease recurrence. Cure models separately investigate the impact of covariates on 1) the proportion of cured individuals and 2) their impact of failure times of non-cured individuals. The former is often the main interest in paediatric oncology. The estimates have a clear clinically meaningful interpretation congruent to binary regression and survival analysis. However, Cure-models fail when there are no long-term survivors or cures and the follow up is inadequate.

Thus, Cure models can provide additional insights. They enable the investigation of predictors for long-term survival without relying on the proportional hazard assumption and should thus be considered more often.

\section{2}

Modelling the GvHD-GvL effect: statistical approaches incorporating clinical aspects

L.C. de Wreede

LUMC (Leiden, NL)

The immunotherapeutic potential of allogeneic haematopoietic stem cell transplants (SCT), summarized as 'Graft-versusLeukemia'-effect (GvL), is essential to current practice, and especially exploited in the contexts of Donor Lymphocyte Infusions (DLI) and Reduced Intensity Conditioning. GVL is strongly associated with Graft-versus-Host-Disease (GvHD). Therefore, in clinical studies GvL can best be analysed through the impact of acute and chronic GvHD on relapse incidence (RI) and nonrelapse mortality (NRM). This talk will present several issues to take into account in such studies.

Firstly, the relevance, potential and possible disadvantages of using data from the EBMT registry will briefly be discussed. We will describe some methods to reduce missingness in these data.

Secondly, several clinical considerations leading to different modelling choices will be discussed: how to separate aGvHD from cGvHD? How to model different degrees of GvHD? Is it possible to separate the impact of GVHD and immunosuppressive treatment? How should we classify patients who receive a second SCT or a DLI? How do we compare patients with different diseases, patient and treatment characteristics?

Thirdly, we will present two statistical models and several refinements to analyse these data. The first of these is the Cox model for the cause-specific hazards for RI and NRM in which both aGvHD and cGvHD are entered as time-dependent variables. This can be extended to a model with time-dependent effects of the occurrence of GVHD or to a landmarking approach in which prediction starts only some time after SCT.

The second model is the multi-state model, in which not only the impact of GvHD on RI and NRM can be studied, but also, e.g., the probability of being alive with cGvHD compared for patients with different baseline characteristics. Such a model also allows to study new outcomes, such as "time spent free from immunosuppression and without relapse" as a measure of treatment success.

Some concrete examples will be shown based on work done jointly with Martin Stern, Johannes Schetelig, Ronald Brand and Hein Putter. 


\section{Paediatric Transplantation, Malignant Diseases}

\section{3}

Do we benefit from second transplants in children diagnosed with AML and MDS/JMML?

M. Mielcarek, J. Owoc-Lempach, E. Gorczynska, M. Ussowicz, D. Kulej, J. Musial, A. Dyla, J. Porwolik, A. Chybicka, K. Kalwak Silesian Piasts University of Medicine (Wroclaw, PL)

Treatment of children with AML or MDS/JMML relapsing after autologous or allogeneic stem cell transplant remains a challenge. The aim of our study was the outcome analysis of paediatric patients, who underwent subsequent transplants (Tx) after relapse(s). One hundred ninety-two transplants (132 single Tx, 21 double Tx, 6 triple Tx) were performed in 159 children (age $9.1 \mathrm{mo}$ - 17.8 yr, Me 9.1 yr) between 1995 and 2011 (follow up: Me 2.8 yr, max. $14.9 \mathrm{yr})$. Patients were diagnosed with AML $(n=94)$, MDS $(n=39)$, JMML $(n=20)$ and sAML $(n=6)$. Seventy-six MUDTx (39.6\%), 45 MSD-Tx (23.4\%), 43 autologous Tx (22.4\%) and 28 haploidentical Tx (14.6\%) were carried out. PB was the graft source in $131 \mathrm{Tx}(68.2 \%), \mathrm{BM}$ in $55 \mathrm{Tx}(28.6 \%), \mathrm{BM}+\mathrm{PB}$ in $5 \mathrm{Tx}(2.6 \%)$ and $\mathrm{CB}$ in $1 \mathrm{Tx}$. Busulfan-containing regimens were applied in 148 procedures $(77.5 \%)$, Treosulfan-containing regimens were used in $27 \mathrm{Tx}(14.1 \%)$ and other incl. RIC were performed in $16 \mathrm{Tx}(8.4 \%)$. Majority of subsequent Tx were performed using Treosulfan-containing regimens $(59.2 \%, \mathrm{~N}=16)$. No statistical differences were detected between studied groups (single Tx, double Tx and triple Tx) acc. to age at Tx, gender, disease, donor type, CD34+/kg recipient body weight, cause of death, ANC and platelet recovery except for graft source (PB was used more frequent in the group with $>1 \mathrm{Tx}$ when compared to the group with single Tx: $81.5 \%$ vs. $62.9 \% ; p=0.036$ ). Both overall survival (OS) and disease-free survival (DFS) were comparable between the group with single $\mathrm{Tx}$ and the group with 2 or $3 \mathrm{Tx}$ (OS: $59.2 \pm 4.5$ for single Tx, $44.2 \% \pm 11.4$ for 2 nd Tx and $66.7 \% \pm 19.2$ for 3 rd Tx; DFS: $62.1 \% \pm 4.5$ for single Tx, $43.6 \%$ \pm 11.3 for $2 \mathrm{nd}$ Tx and $50.0 \% \pm 20.4$ for 3rd Tx). The frequency of aGvHD III-IV was comparable among groups $(22.0 \%$ for single Tx, $0.0 \%$ for $2 \mathrm{nd} \mathrm{Tx}$ and $16.7 \%$; for $3 \mathrm{rd} \mathrm{Tx}, \mathrm{p}=0.173$ ) as well as for chronic GvHD (19.7\% for single Tx, 28.6\% for 2nd Tx and $16.7 \%$ for $3 r d T x, p=0.628$ ). There were no relevant differences between limited and extensive chronic GvHD between the group with $1 \mathrm{Tx}$ and the group with $>1 \mathrm{Tx}$. OS in relation to the disease type was as presented: $\mathrm{sAML}: 66.7 \% \pm 19.2 ; \mathrm{N}=6, \mathrm{AML}: 62.8 \% \pm 5.3 ; \mathrm{N}=94$, MDS: $57.1 \% \pm 8.2 ; \mathrm{N}=39, \mathrm{JMML}: 32.8 \pm 11.0 ; \mathrm{N}=20, \mathrm{p}=0.152$. Subsequent transplants using reduced toxicity regimens (incl. Treosulfan) offer a curative option for children with AML or MDS/JMML relapsing posttransplant. Haploidentical transplants remain an alternative only for selected pts, for whom unrelated donors are not available.

\section{O244}

Peripheral blood stem cell transplant to support dosimetry guided higher dose molecular radiotherapy with co-administration of topotecan as a radiosensitiser for metastatic neuroblastoma. A SIOPEN study R. Ladenstein (1), J. Bomanji (2), M. Hoffmann (3), A. Forjaz De Lacerda (4), F. Saran (5), A. Uyttebroeck (6), A. Staudenherz (3), A. Alspach (7), R. Mairs (8), G. Flux (5), M. Gaze (2) (1)CCRI-St. Anna Kinderspital (Vienna, AT); (2)University College London Hospitals NHS Foundation Trust (London, UK); (3)Medical University of Vienna (Vienna, AT); (4)Instituto Portugues de Oncologia de Lisboa Francisco Gentil, E.P.E. (Lisbon, PT); (5)Royal Marsden NHS Foundation Trust (Sutton, UK); (6)UZ Leuven, University Hospitals Leuven (Leuven, BE); (7)Children's Cancer Research Institute (Vienna, AT); (8)University of Glasgow (Glasgow, UK)

Objectives: The principal toxicity of molecular radiotherapy with 131-lodinemeta-iodobenzylguanidine ( $\mathrm{mlBG}$ )isdose-dependent myelosuppression. Conventional mIBG therapy gives a whole body radiation absorbed dose (WBD) of around 2Gy, which allows spontaneous count recovery. This study evaluates the use, in a multi-centre setting, of peripheral blood stem cell (PBSC) support to allow safe dosimetry guided escalation of the administered activity of mIBG, given together with topotecan, also myelosuppressive, as a radiosensitizer, to give a prescribed WBD, guided by in vivo dosimetry, of $4 \mathrm{~Gy}$ in two fractions.

Methods: Patients with refractory or relapsed high risk neuroblastoma underwent PBSC harvest. The treatment schedule of mIBG and topotecan in neuroblastoma, MATIN, incorporates two administrations of mIBG prescribed to give a WBD of 4 Gy, with concomitant topotecan. PBSC return was when the residual radioactivity fell below $30 \mathrm{MBq}$, around two weeks after the second $\mathrm{mIBG}$ administration.

Results: MATIN has been used 5 European centres in 69 patients with neuroblastoma, 44 male, 25 female, median age 6 years. 46 patients had refractory or progressive disease without prior myeloablative therapy (MAT), 23 had relapsed after MAT.

Toxicity: In 2 patients, the full treatment was not given because of adverse events. There was 1 treatment-related death. 5 patients failed to regain normal platelet counts.

Further therapy: In $61 \%$ of refractory patients, further potentially curative treatment including MAT was delivered. In six patients, repeat MATIN was given. Survival: For all patients, three year event free survival (EFS) was $0.16( \pm 0.05)$ and overall survival (OS) was $0.26( \pm 0.06)$. For patients without prior MAT, EFS and OS were $0.25( \pm 0.07)$ and $0.37( \pm 0.09)$. For relapsed patients, EFS was 0 and OS $0.07( \pm 0.07)$. These differences are statistically significant $(\mathrm{P}=0.002)$

Conclusion: We have demonstrated that the MATIN schedule has an acceptable morbidity and mortality profile in a group of neuroblastoma patients with a very poor prognosis. In very high risk patients with refractory disease, MATIN enabled further, potentially curative, treatment to be given resulting in encouraging survival rates. The MATIN schedule will be further evaluated in a randomised trial.

\section{5}

Association between busulfan exposure and outcome in children receiving intravenous busulfan before haematopoietic stem cell transplantation

M. Ansari, Y. Théoret, M. Rezgui, S. Mezziani, C. Desjean, M. Vachon, M. Labuda, M. Champagne, M. Duval, C. Peters, M. Krajinovic, $\mathrm{H}$. Bittencourt on behalf of the Pediatric Disease Working Parties of the European Blood and Marrow Transplant group

Busulfan $(\mathrm{Bu})$ is a key compound in conditioning regimens $(\mathrm{CR})$ of children undergoing hematopoietic stem cell transplantation (HSCT). Inter-individual differences in Bu levels may cause treatment failure and toxicity. Intravenous (iv) Bu combined with therapeutic drug monitoring-guided dosing is associated with higher event-free survival (EFS), lower transplant-related mortality (TRM) and toxicity. Nevertheless, optimal target area under the curve (AUC)/steady state concentration (Css) in children undergoing HSCT remains unclear in $\mathrm{CR}$ with iv Bu. Ninety-five patients were studied from 2000 to 2010 in St Justine Hospital, Canada with iv $\mathrm{Bu}$ dose adjustment to target Css of 600-900 $\mathrm{ng} / \mathrm{ml}$. Median age was $8.1(0.1-20)$ years and 60 patients had a diagnosis of malignant disease. Twenty-nine patients received a graft from a sibling donor and 63 from an unrelated donor; 58 patients received a cord blood as graft source. After first dose of iv $\mathrm{Bu}$, median maximum concentration (Cmax) was $844 \mathrm{ng} / \mathrm{ml}$ (537-1338), Cmin $215 \mathrm{ng} / \mathrm{ml}$ (66.4-522), Cmean $500 \mathrm{ng} / \mathrm{ml}$ (277-870), Css 603 ng/ml (295-1227), AUC 205585 $\mathrm{min} x \mathrm{ng} / \mathrm{ml}$ (94859-431260), and clearance $4.1 \mathrm{ml} / \mathrm{min} / \mathrm{kg}$ (1.84-7.68). Median \% adjustement of iv Bu dose was $+15.9 \%$ (dose increased in 57 and dose decreased in 11 patients). Neutrophil, and platelet recoveries, grade 2-4 acute GVHD, and TRM, incidence were $91 \%, 88 \%, 9 \%$ and $8 \%$, respectively. 


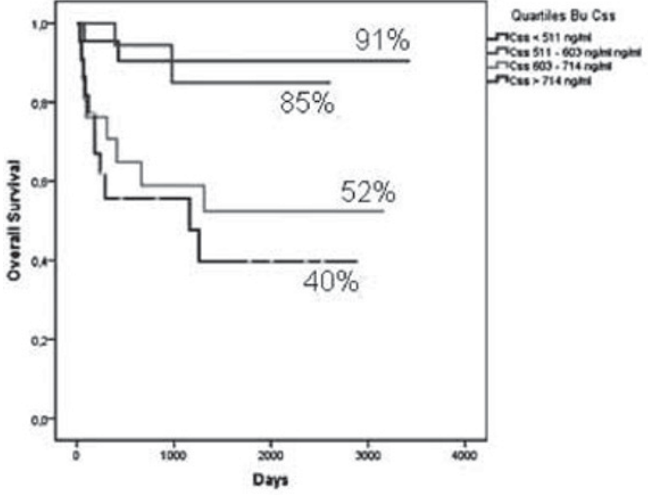

Relapse incidence was $37 \%$. Incidence of VOD, hemorrhagic cystitis $(\mathrm{HC})$ and lung toxicity were $11 \%, 26 \%$ and $9 \%$, respectively. In univariate analysis, a Bu Css higher than the median $(>603 \mathrm{ng} / \mathrm{ml})$ was associated with higher TRM $(P=0.01)$, and $\mathrm{HC}(\mathrm{P}=0.03)$ incidences. Overall (OS) and event-free survivals (EFS) were $69 \%$ and $55 \%$. Bu Css influenced OS and EFS in univariate analysis with a lower OS and EFS with a higher Css $(P<0.001$ for both - Figure 1). In multivariate analysis, a higher $\mathrm{Bu}$ Css was associated with a higher TRM $(\mathrm{HR}=2.76 ; \mathrm{P}<0.001)$, a lower EFS $(H R=1.88 ; P<0.001)$ and $O S(H R=2.12 ; P=0.001)$. Our study suggests that outcome of pediatric patients receiving iv Bu can be predicted according to its pharmacokinetics. A prospective multicentric EBMT study is ongoing to confirm these results. A reduced first dose strategy should be investigated as well as the impact of pharmacogenetics.

\section{6}

Glutathione S transferase gene variations influence busulfan pharmacokinetics and outcome of haematopoietic stem cell transplantation

M. Krajinovic, M.A. Rezgui, Y. Théoret, M. Duval, S. Meziani, J. Rousseau, M. Labuda, C. Pryzbila, M. Vachon, C. Desjean, M. Champagne, C. Peters, H. Bittencourt, M. Ansari on behalf of the Pediatric Disease Working Parties of the European Blood and Marrow Transplant group

Busulfan (BU) is a key compound in conditioning myeloablative regimens for children undergoing hematopoietic stem cell transplantation (HSCT). There are wide interindividual differences in $\mathrm{BU}$ pharmacokinetics, which might increase the risk of venoocclusive disease (VOD), graft rejection and disease relapse. As BU is mainly metabolized by glutathione S-transferase (GST), it is possible that functional polymorphisms in GST genes may contribute to the variability in $\mathrm{BU}$ pharmacokinetics and/or HSCT outcomes. Our recent pilot study interrogating the polymorphisms in GST gene and pharmacokinetics after the first dose of intravenous BU showed a significant association between GSTM1 null genotype and higher drug exposure. The objective of the present study was to extend this analysis to higher number of patients $(n=74)$, to evaluate the association between GSTA1, GSTP1 and GSTM1 genotypes and drug exposure and to evaluate whether GST genotype affect HSCT outcome in terms of efficacy and toxicity. The genotypes in GSTA1 (C-69T, A-513G, G-631T, C-1142G), GSTM1 (deletion) and GSTP1 gene (A1578G, C2293T) were obtained and association analyses were performed with individual polymorphisms as well as with GSTA1 haplotypes resolved by PHASE software.

Results seem to successfully replicate those obtained in pilot study for GSTM1 genotype. GSTA1 and GSTP1 polymorphisms analyzed individually were not associated with

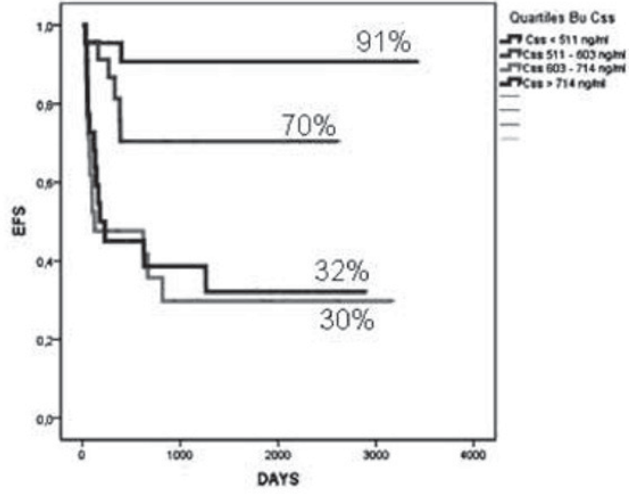

first dose BU pharmacokinetics. However, haplotype analysis indicated that particular GSTA1 haplotypes affect drug exposure. The haplotype *A2 (C-1142, G-631, A-513, C-69), found in $23 \%$ of patients, was associated with lower drug levels, lower dose adjusted drug concentrations (PAUC $=0.02$; $P C \max =0.003$; PCss $=0.02$ ) and higher drug clearance $(p=0.007)$. This finding is in accordance with functional studies suggesting higher GSTA1 enzymatic activity in GSTA1 ${ }^{*} A$ carriers. Gene-dosage effect was also observed with drug exposure and clearance further affected in additive manner (PAUC=0.009; PCmax=0.003; PCss, =0.01, PCl=0.004). Patients with two copies of haplotype *A2 had better event free survival $(p=0.04)$.

In contrast, homozygous individuals for the GSTA1 T-69 or G-1142 allele defining haplotype * $\mathrm{B}$ and haplotype *B1, respectively, had higher occurrence of VOD ( $H R=10.5, p=0.002)$. The results obtained open the possibility to tailor the first BU dose according to patient genetics.

0247

Treosulfan pharmacology in children: results of a feasibility study

R. Beier(1), R. Hilger(2), P. Sedlacek(3), J. Greil (4), M. Sauer(1), K.-W. Sykora (1)

(1)Hannover Medical University (Hannover, DE); (2)University Hospital Essen (Essen, DE); (3)Charles University Motol (Prague, CZ); (4)Heidelberg University (Heidelberg, DE)

Treosulfan (TREO) is increasingly used in pediatric HSCT because of its low acute toxicity. Little is known about the optimal dose required for children of different age groups. We conducted a pharmacology feasibility study to identify logistical and practical problems, especially because infants were to be included in the study.

Transplantations: 15 children from 3 centers were included. 7 children under $10 \mathrm{~kg} \mathrm{BW}$ (age 3.8-17.7, mean 10mo) received $3 \times 12 \mathrm{~g} / \mathrm{m}^{2}$ and 8 children over $10 \mathrm{~kg}$ BW (age 1y4mo-14y, mean $5 \mathrm{y} 2 \mathrm{mo}$ ) received $3 \times 14 \mathrm{~g} / \mathrm{m}^{2}$ of TREO over 2 hours on 3 consecutive days. TREO was measured at 8 different times (0-12 h) after administration on days 1 and 3 . The "pushpull" method was used for blood sampling to minimize blood loss to $0.5 \mathrm{ml}$ per time point. A method to measure TREO concentration in $0.5 \mathrm{ml}$ of diluted plasma was established and validated. Urine was collected for renal TREO excretion. Initial pharmacokinetic data were obtained.

Feasibility issues identified: Obtaining exactly $0.5 \mathrm{ml}$ of blood and pipetting it quickly into the prepared $0.5 \mathrm{ml}$ of acid citrate solution was more difficult in practice than expected, leading to inexact pipetting. Nevertheless, the correct concentration of TREO could still be calculated from the total sample volume. When the push-pull method was used on central 
venous catheters, frequent catheter malfunctions and occlusions were seen, especially in infants. Quinton type implanted catheters were unproblematic.

Pharmacology: The mean AUC in infants receiving $3 \times 12 \mathrm{~g} / \mathrm{m}^{2}$ TREO was higher than in the older children receiving $3 \times 14$ $\mathrm{g} / \mathrm{m}^{2}$ (2203 vs $1507 \mathrm{mg} / \mathrm{l} \times \mathrm{h}, \mathrm{p}=0.04$ ). Volume of distribution at steady state (Vss) was lower in infants (9.4 vs 16.2l, $p=0,02$ ) than in older children. Half-life (mean 1.31 vs $1.59 \mathrm{hrs}$ ), percentage of renal elimination (60.2 vs $59.4 \%$ ), and mean residence times ( 2.79 vs $2.94 \mathrm{hrs}$ ) were not different. There was no drug accumulation between days 1 and 3 owing to the low half-life of TREO that resulted in negligible trough levels. AUC profiles on days 1 and 3 were almost superimposable.

Conclusion: A TREO pharmacology study in children that includes infants is feasible in a multicentric setting using the methods and considering the caveats described above. The high AUCs and low vss seen in infants compared to older children underline the necessity of further investigations that also include clinical parameters. Supported by BMBF grant Nr. 01E00802 IFB-TX Hannover and Medac GmbH.

\section{8}

Anti-HLA antibodies and outcome in paediatric cord blood transplantation

M. Ansari (1), C. Uppugunduri (1), H. Bittencourt (2), F. GumyPause (1), S. Ferrari-Lacraz (1), Y. Chalandon (1), H. Ozsahin (1), J.M. Tiercy (1), M. Duval (2), M. Krajinovic (2), J. Villard (1) (1)Hôpital Universitaire (Geneve, CH); (2)CHU St Justine (Montreal, CA)

The development of solid phase assay for detection of antiHLA antibodies has revolutionized our understanding of the role of these antibodies to predict antibody mediated rejection and graft survival in solid organ transplantation. In haematopoietic stem cell transplantation, influence of anti-HLA antibodies on graft outcome is still a matter of debate. Because of an adequate HLA matching in most cases between recipients and donors, incidence of donor specific anti-HLA antibodies (DSA) is low. Development of cord blood transplantation (CBT) with low level of HLA matching strongly increases the risk to detect the presence of anti-HLA antibodies by using high sensitive techniques. Presence of DSA might be associated with graft failure, delayed engraftment or persistant thrombocytopenia that could jeopardizes global survival. In pediatric patients, very limited data is available. In this study, we analyzed the presence of anti-HLA antibodies in a cohort of 55 children receiving a CBT with a conditioning regimen of Busulfan and Cyclophosphamide. Using luminex screening assays, we have detected before CBT the presence of anti-HLA antibody in $42 \%$ of recipients $(23 / 55)$ among them $15(27 \%)$ were positive for class I, 17 (31\%) were positive for class II and 9 (16\%) were positive for both anti-HLA antibodies. Each positive test was confirmed by an analysis of anti-HLA antibody specificity by luminex single antigen assay. The titer of these antibodies was defined using the Mean Fluorescence Intensity (MFI). In 8 patients, anti-HLA antibodies were directed against HLA of the donor.

Presence of anti-HLA antibody class I significantly influenced neutrophil recovery $(p=0.021, O R=13.09)$. Platelet recovery showed a tendency to be prolonged in the presence of anti$\mathrm{HLA}$ antibodies of class II, with MFI>2,000 $(p=0.06)$. Interestingly, presence of DSA was associated with a higher incidence of acute GvHD ( $p=0.03)$. Finally, overall survival after CBT was not affected by anti-HLA antibody except in presence of antiHLA class II but at high MFI $(>5,000, p<0.001)$.

In summary, our data suggests that anti-HLA antibodies of class I and class II are associated with significant clinical events. A therapeutical strategy to reduce the level of anti-HLA antibody before CBT in children should be considered.

\section{9}

Acute and chronic GvVHD in paediatric patients treated by ECP, a 12-year surveilance

V. Witt (1), A. Lawitschka (1), S. Matthes (1), C. Peters (1), B. Sailer (1), G. Fritsch (2)

(1)St. Anna Kinderspital (Vienna, AT); (2)St. Anna Kinderkrebsforschungsinstitut (Vienna, AT)

Objectives: Graft-versus-host disease (GVHD) is one of the major complications influencing morbidity and mortality after allogenic stem cell transplantation (SCT). In this retrospective analysis over 10 years, we evaluate the outcome of extracorporeal photochemotherapy (ECP) as a treatment modality of GVHD.

Patients and Methods: From 1999 to 2011, 38 patients, consisting of $15(\mathrm{f} / \mathrm{m} 7 / 8)$ with acute GVHD (aGVHD) and $23(\mathrm{f} / \mathrm{m}$ 11/12) with chronic GVHD (cGVHD), were treated with ECP. Patients weighing less than $40 \mathrm{~kg}$ were available for the offline system $(n=16)$. Very small or critically ill children were treated with mini $\operatorname{ECP}(n=1)$. And additionally there was the closed or offline system for patients weighing more than $40 \mathrm{~kg}(\mathrm{n}=12)$. Some patients were treated with both the inline and offline system $(n=7)$ or with first mini ECP followed by the offline method $(n=2)$. The median age at the first ECP was 7 years for patients with aGVHD and 13 years for patients with cGVHD, the median body weight was 27 and 38 kilograms. Fifteen patients were suffering from aGVHD with manifestations in skin $(n=14)$, oral mucosa $(n=3)$, liver $(n=6)$ and gut $(n=4)$. Twenty-three patients had ECP because of their cGVHD, the affected tissues were: skin $(n=17)$, oral mucosa $(n=16)$, liver $(n=8)$, gut $(n=4)$, lung $(n=4)$, joints $(n=10)$, eyes $(n=9)$, muscles $(n=2)$ and the central nervous system $(n=1)$.

Results: In a GVHD 11/15 (73\%) patients responded with improvement overall and $11 / 14(79 \%)$ in skin, $0 / 3(0 \%)$ in oral mucosa, 4/6 (67\%) in liver and 3/4 (75\%) in gut involvement after a median of 15 cycles. Chronic GVHD improved in 18/23 $(78 \%)$ patients. Skin improved in $13 / 17(76 \%)$, liver in $7 / 9$ $(88 \%)$, gut in $2 / 4(50 \%)$ and lung in $1 / 4(25 \%)$ after a median of 26 cycles. The overall survival was $89 \%$ and $94 \%$ for patients responding to ECP and $0 \%$ and $40 \%$ for non-responders.

Conclusion: Outcome is dependent on response to ECP. ECP enables steroid tapering and shows good responses for acute and chronic GVHD. In spite of using different methods to perform ECP, the outcome doesn't seem to be strongly dependent on it. Even after 10 years the survivors of GVHD, treated by ECp showed no specific side effects regarding to ECP. ECP in children seems to be very save, even in long term outcome.

\section{Multiple Myeloma / Chronic Leukaemia / Myelodysplastic Syndromes}

\section{0}

Phase II study of azacitidine (Vidaza ${ }^{\circledR}$, Aza) and donor lymphocyte infusions as first salvage therapy in patients with acute myeloid leukaemia or myelodysplastic syndromes relapsing after allogeneic haematopoietic stem cell transplantation

T. Schroeder (1), A. Czibere (1), N. Kröger (2), U. Platzbecker (3), G. Bug (4), L. Uharek (5), T. Luft (6), C. Wolschke (2), R. Fenk (1), R. Haas (1), G. Kobbe (1)

(1)University Hospital Düsseldorf (Düsseldorf, DE); (2)University Hospital Hamburg (Hamburg, DE); (3)University Hospital Dresden (Dresden, DE); (4)University Hospital Frankfurt (Frankfurt, DE); (5)University Hospital Berlin Charite (Berlin, $D E)$; (6)University Hospital Heidelberg (Heidelberg, DE)

Background: Relapse after allo-SCT in pts with AML or MDS is associated with a poor prognosis and therapeutic options are limited. Thus, there is need for novel strategies which ideally target the leukemic clone and enhance a GvL effect. Aza might 
provide these properties, and retrospective studies in this context were encouraging.

Design/Methods: We here report the final results from a prospective single-arm EBMT phase II trial investigating the combination of Aza+DLI as 1st salvage therapy in pts with AML or MDS with hematological relapse after allo-SCT. Treatment contained up to 8 cycles Aza (100 mg/m²/d d1-5, every $28 \mathrm{~d}$ ) followed by DLI with increasing dosages $\left(1-5 \times 10^{6}-1-5 \times 10^{8} \mathrm{cells} / \mathrm{kg}\right)$ after every 2ndAza cycle.

Results: A total of 30 pts (19 female/11 male, median age 55 y) from 6 centres were included. The majority of pts $(n=28)$ suffered from AML, while 2 pts had MDS or MDS/MPN. At transplant, 16 pts (53\%) had active disease and 14 pts (47\%) were in remission.

Following allo-SCT, hematological relapse evolved in all pts (median BM blasts: $34 \%$, median chimerism: $67 \%$ ) at a median time of $175 d$ (19-1699).

A median of 3 courses Aza (range 1-8) were administered, and 22 of 30 pts $(73 \%)$ received DLI (median: 1 , range: $1-4$, median CD3 dose $5 \times 106 / \mathrm{kg} / \mathrm{DLI})$.

ORR was $47 \%$ (CR/CRi 7 pts/ PR 2 pts SD 5 pts). Median time and number of Aza cycles to best response were $79 \mathrm{~d}$ (range 28-299) and 3 cycles (range 1-8). Of the 7 pts with CR, 5 pts continue in CR for a median of $777 \mathrm{~d}$ (range 461-890), while 1 pt relapsed after $396 \mathrm{~d}$ and $1 \mathrm{pt}$ died from GvHD.

By Dec 2011 median follow-up of surviving pts is $817 \mathrm{~d}$ (range 732-974) and 5 pts (17\%) are currently alive. Twelve pts have died due to progressive disease, while 7 pts died during (2 infection, 1 bleeding) or after the end of therapy (1 GvHD, 2 infection, 1 bleeding). Median OS of all pts is $117 \mathrm{~d}(66-168)$. Patients with $\mathrm{CR} / \mathrm{CR}$ i had a significant longer OS than pts not reaching CR/CRi (n. r. vs. $83 \mathrm{~d}, \mathrm{p}<.001$ ). A lower blast count and adverse cytogenetics positively correlated with the likelihood of achieving CR.

Eleven pts $(37 \%)$ developed aGvHD, while cGvHD was observed in 5 pts (17\%). Cytopenias grade III/IV occurred in all pts, but were considered to be drug-related in only 11 pts (37\%). The most common drug-related grade III/IV non-hematological toxicity were infections.

Conclusion: Aza and DLI as 1st salvage therapy is safe and active in pts with AML/MDS who relapse after allo-SCT inducing durable remissions in a subgroup of pts.

\section{1}

Impact of azacitidine prior to allogeneic stem cell transplantation for myelodysplastic syndromes: a largescale study on behalf of the SFGM-TC and GFM groups

G. Damaj, A. Duhamel, M. Robin, M. Michallet, M. Mohty, V. Stéphane, Y. Beguin, A. Garnier, B. Lioure, J. El Cheikh, N. Fegueux, J. Cornillon, L. Adés, S. François, M.-T. Rubion, A. Huyn, J. Marolleau, S. Park, F. Garnier, P. Fenaux, I. YakoubAgha for the SFGM-TC

AZA has become a reference tttof IPSS higher risk MDS, but its roleremain uncertain.We report on 462 consecutive MDS pts who underwent SCT between 1999-2009. Inclusion criteria were age $>18$, dg of MDS according to the FAB or WHO criteria, SCT from sibling $(n=254)$ or MUD HLA $(10 / 10(n=163))$. The SC source was blood $(n=294)$ or marrow $(n=123)$ and the conditioning was either myeloablative $(n=184)$ or of reduced intensity $(n=233)$.

462 files were analyzed. 255M,162F. At dg, WHO was, $112 \mathrm{RA} /$ RARS/RCMD,137 RAEB1,140 RAEB2 and 28 RAEBt/AML; Cytogenetic IPSS were fav $(n=215)$, interm $(n=106)$, unfav $(n=91)$; 204 pts IPSS Int-2 or High. 144pts had progressed before allo-SCT. Prior to SCT, 66 pts had received AZA, alone $(n=49)$, $A Z A+I C T(n=17)$. The 328 remaining pts received BSC $(n=171)$ or ICT $(n=180)$. In AZA groups, the drug was started 38 to $941 \mathrm{~d}$ after $\mathrm{dg}$ and discontinued 6 to $438 \mathrm{~d}$ before SCT with a median number of 4 cycles. 288 patients were considered responders at SCT, while 242 were SCT with progressive disease. Median age at SCT was 54 years (18.3-70.7). Pts received myeloabla- tive $(n=150)$ or nonmyeloablative (NMA) $(n=267)$ conditioning with BM $(n=123)$ or PBSC $(n=294)$ as source SC. Compared with other treatment groups, patients belonging to AZA-alonegroup were older $(p=.0001)$, had more often Int- 2 and high IPSS $(p=.0001)$ and one third of them $(33 \%)$ had poor cytogenetics $(p=.07)$. They received more often NMA conditioning $(p=.0001)$ from MUD $(p=.0007)$.

As of April, 2011, median FU was 48 mo (2.4-146.4. The estimated $3-y$ OS and EFS were $59 \%, 35 \%, 45 \%, 53 \%(p=.10)$; and $53 \%, 29 \%, 40 \%, 54 \%(p=.11)$ in the AZA-alone, AZAchemo, iCT and BSC groups, respectively. The estimated 3yTRM was 35\%, 21\%, 34\% and 28\% ( $p=.036)$ and $3 y$ relapse was $31 \%, 35 \%, 38 \%$ and $27 \%$ in the AZA-alone, AZA-chemo, iCT and BSC groups $(p=.54)$. The $3 y$ OS and EFS AZA group were better than the iCT alone; the difference was not statistically significant. This difference was statistically different when we compare the 3-y OS and EFS between the AZA group and $A Z A+i C T$ with $P=0.027$ and 0.032 respectively.

Multivariate analyses confirmed the influence of prior ttt with an unfav outcome in pts in AZA-chemo group on OS and EFS $(p=.027$ and $p=.032$, respectively).

AZA ttt is valid therapeutic approach, but mainly in pts receiving AZA alone with an outcome at least equivalent to pts receiving induction CT prior SCT. SCT in pts who required AZA+CT had less satisfactory outcomes, possibly reflecting additional toxicity and/or more resistant disease.

\section{2}

First evidence that alloSCT can improve the natural course of poor-risk chronic lymphocytic leukaemia as defined by the EBMT consensus criteria: a retrospective donor versus no donor comparison

I. Herth, U. Hegenbart, S. Dietrich, M. Rieger, P. Stadtherr, A. Bondong, H. Tran, T. Zenz, A. Ho, P. Dreger

University of Heidelberg (Heidelberg, DE)

In order to study if alloSCT can improve the dismal natural course of poor-risk CLL, we aimed at assessing the impact of alloSCT by measuring its outcome from the time of donor search indication rather than from the time of transplant, thereby including those patients who failed to proceed to allografting for any reason.

Study and Design and Patients: In a single centre retrospective analysis, course and outcome of all patients with CLL referred for evaluation of alloSCT indication between June 2005 and July 2011 were recorded. Primary endpoint was overall survival (OS) of those patients for whom a $9 / 10$ or $10 / 10$ matched donor could be found within 3 months compared with that of patients without donor, measured from the 3-month landmark after donor search initiation.

Results: Altogether 117 patients with a median age of 53 (37-69) years were referred in the six year time period. An indication for donor search was seen in 98 patients $(84 \%)$. According to EBMT criteria, indication was (1) symptomatic $17 \mathrm{p}$ - in 31 patients, (2) fludarabine refractoriness in 27 , and (3) early relapse after intensive pretreatment in 35 patients. Another 5 patients were considered because of Richter's transformation. Of the 98 patients with indication, 8 patients refused donor search. A donor could be identified for $72(80 \%)$ of the remaining 90 patients. Of these, $21(29 \%)$ did not proceed to transplant because of CLL progression (9) or other reasons (12), whereas 51 patients underwent RIC alloSCT from a sibling (17) or unrelated donor (34). With a median observation time of 20 (0-73) months, OS at 2 years from start of search was $69 \%$ for all 98 patients who had a search indication. There was no impact of type of EBMT criterion (1 vs 2 vs 3 ) and age on the outcome of patients with search indication. With regard to the primary endpoint, OS at 2 years from the 3-month landmark after start of search (excluding all patients who died within this time frame for any reason) was significantly better in patients with a donor than in those without $(83 \%$ vs $56 \%$; $p=0.007)$. 2 -year OS of the 51 patients actually transplanted $91 \%$. 
Conclusions: Survival of patients with poor-risk CLL and alloSCT indication according to the EBMT consensus for whom a donor cannot be found is significantly inferior compared to patients with a donor. This observation provides first comparative evidence that alloSCT indeed may have the potential to improve the natural course of poor-risk CLL.

\section{OS from 3-month landmark by donor availablility}

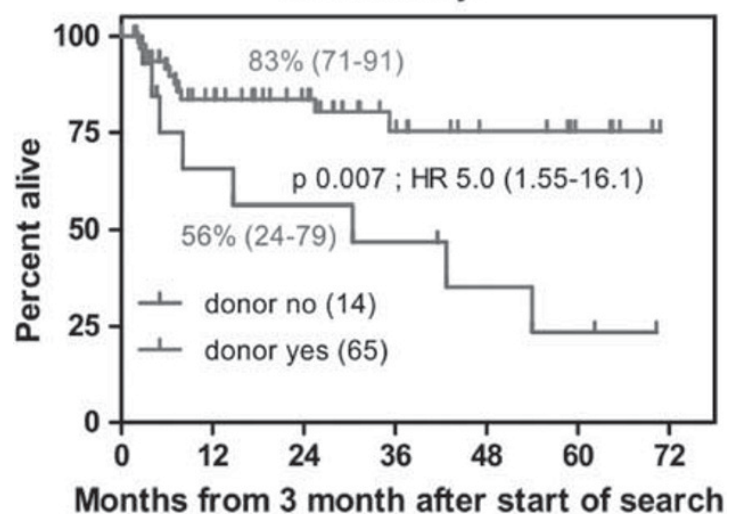

\section{3}

Treatment with alemtuzumab prior to allogeneic stem cell transplantation in patients with advanced chronic lymphocytic leukaemia - results of a prospective phase II trial (NCT 00337519)

J. Schetelig, M. Bornhäuser, C. Thiede, A. Kiani, U. Platzbecker B. Mohr, U. Oelschlägel, M. Hänel, H. Baurmann, K. Kolbe, M. Kaufmann, M. Zeis, K. Schäfer-Eckart, G. Ehninger on behalf of the German Cooperative Transplant Study Group

Chemotherapy-refractory stages of chronic lymphocytic leukemia (CLL) represent an indication for allogeneic hematopoietic cell transplantation (HCT). Indirect evidence exists, that lower tumor load is associated with better outcome. Alemtuzumab has demonstrated activity in chemo-refractory disease and to prevent graft-versus-host disease (GVHD). Yet, treatment with alemtuzumab prior to HCT has been associated with inferior outcome in one large retrospective analysis. Here, results from a phase II trial are presented where we systematically investigated treatment with alemtuzumab followed by a washout period and subsequent reduced intensity conditioning (RIC) prior to allogeneic HCT.

Methods: All patients were scheduled for one month of cytoreductive pretreatment with $3 \times 30 \mathrm{mg}$ alemtuzumab weekly. The washout period after the last dose of alemtuzumab was increased from two weeks to one month during the study. Antibody levels were measured on the day of HCT and T-cell engraftment was monitored. RIC consisted of fludarabine (150 $\mathrm{mg} / \mathrm{m} 2)$ and busulfan $(8 \mathrm{mg} / \mathrm{kg})$ in combination with CSA $/$ MTX as GVHD-prophylaxis.

Results: 62 patients with relapsed or refractory CLL and a median age of 56 years were included. Twenty-four patients (39\%) suffered from refractory disease, 12 patients $(19 \%)$ had a del(17p), 19 patients (31\%) had treatment failure within 2 years. Two patients died early after pre-treatment due to progressive disease and did not proceed to HCT. Donors were HLA-identical siblings for 26 and HLA-compatible unrelated donors (one mismatch accepted) for 34 patients. No primary or secondary graft failure occurred. Interference of persisting antibody levels with T-cell engraftment could be demonstrated. The cumulative incidence of acute GVHD grades II to IV at day +100 was $42 \%$ and of extensive chronic GVHD at one year was $56 \%$.

With a median follow-up of 35 months (16 to 61 months) 3-year overall survival and progression-free survival were 62\% $(95 \%$ $\mathrm{Cl}, 48 \%$ to $76 \%)$ and $52 \%(95 \% \mathrm{Cl}, 38 \%$ to $66 \%)$. Day +100 non-relapse mortality was $2 \%$. At 3 years non-relapse mortality and relapse incidence were $24 \%(95 \% \mathrm{Cl}, 12 \%$ to $36 \%)$ and $24 \%(95 \% \mathrm{Cl}, 13 \%$ to $35 \%)$, respectively.

Conclusions: The long-term results of this study argue in favor of that strategy for patients with refractory disease. However, unless in vivo T-cell depletion is the goal, a washout period of a minimum of one month after the last dose of alemtuzumab should be kept and T-cell engraftment needs to be monitored.

\section{4}

Comparative efficacy of tandem autologous-autologous versus tandem autologous-reduced intensity allogeneic haematopoietic cell transplantation in multiple myeloma: results of a systematic review and meta-analysis

M. Kharfan-Dabaja (1), M. Hamadani (2), T. Reljic (3), W. Bensinger (4), B. Djulbegovic (5), A. Kumar (5)

(1)American University of Beirut (Beirut, LB); (2)West Virginia University (Morgantown, US); (3) University of South Florida (Tampa, US); (4)Fred Hutchinson Cancer Research Center/ University of Washington (Seattle, US); (5)University of South Florida and Moffitt Cancer Center (Tampa, US)

Background: Despite strides in our understanding of clinical, genetic, and molecular aspects of the disease, coupled with development of more effective therapies, MM remains incurable. Combining benefits of cytoreductive-therapy with adoptive immunotherapy forms the basis of a tandem auto-allo HCT treatment strategy in patients (pts) with MM. Conflicting results, however, have been reported when an auto-allo HCT approach has been compared to an auto-auto HCT strategy, which relies solely on dose-intensity of chemo- or chemoradio-therapy to eradicate disease.

Methods: A systematic search of MEDLINE database (1966Nov 5, 2011), and pertinent conference proceedings, identified 203 publications. Any randomized controlled trial (RCT) comparing an auto-allo HCT to tandem auto-auto HCT were eligible. A meta-analysis was conducted as per the standards recommended by Cochrane Collaboration.

Results: Six RCT (full-length manuscript=4, abstract=2) met inclusion criteria. All studies allocated pts to auto-allo HCT if an HLA-matched sibling donor was available (genetic randomization). The 6 included trials enrolled 1798 pts. Three studies enrolled 1229 (auto-allo=414, auto-auto=815) pts on intention-to-treat (ITT) principle. Three remaining trials reported outcomes on 569 (auto-allo=273, auto-auto=296) pts using a per-protocol approach. In all but one study, groups were balanced for presence of disease-related prognostic factors. Non-relapse mortality (NRM) was significantly worse with an auto-allo HCT approach [risk ratio $(\mathrm{RR})(95 \% \mathrm{Cl})=3.55(2.17$ $5.80), p<0.00001]$. Assessing response rates by achievement of at least a very good partial response was not different among these treatment strategies $[R R(95 \% \mathrm{Cl})=1.03(0.91-1.16)$, $p=0.69]$; but complete remission (CR) rate was significantly higher in pts who received an auto-allo HCT [RR=1.53 (1.211.93), $p=0.0004]$. Event-free survival (EFS) was better in the auto-allo HCT group on per-protocol analysis [HR=0.79 (0.66$0.95)), p=0.01]$ of 4 trials, but did not differ on ITT analysis $[\mathrm{HR}=0.83(0.60-1.15), p=0.26]$ of 3 trials. Overall survival $(\mathrm{OS})$ did not differ among these different treatment strategies whether analyzed on per-protocol [HR=0.87 (0.54-1.40), $p=0.56]$ of 3 trials, or by ITT [HR=0.80 (0.48-1.32), $p=0.39]$ analysis of 3 trials.

Conclusion: Despite higher CR rates, there is no apparent improvement in OS by offering an auto-allo HCT; but this approach results in significantly higher NRM in pts with MM. 


\section{5}

Tandem autologous transplantation versus autologous plus reduced-intensity conditioning allogeneic transplantation in the management of newly diagnosed multiple myeloma: meta-analysis of all prospective trials with biological randomisation

L. Costa, K. Armeson, E. Hill

Medical University of South Carolina (Charleston, US)

Introduction: Autologous transplantation has a defined role in the upfront treatment of multiple myeloma (MM) but nearly all patients will eventually relapse. Allogeneic transplantation carries the benefit of graft versus myeloma (GVM) effect but also high risk of treatment related mortality (TRM). Trials randomizing patients with newly diagnosed MM to tandem autologous (TA) or the combination of autologous + RIC allogeneic transplantation (AR) based on the availability of an HLA-matched donor have yielded heterogeneous results. We performed a meta-analysis of all reported trials comparing TA with AR transplantation in newly diagnosed MM to summarize the available evidence supporting the upfront use AR in multiple myeloma. Methods: We utilized a comprehensive search strategy to identify all trials meeting the following entry criteria: prospective trial, inclusion only of newly diagnosed patients (typically after conventional induction therapy), subjects undergoing similar induction therapy and first autologous transplantation in both arms, assignment to RIC allogeneic transplantation or a second autologous transplantation based exclusively on the availability or not of a HLA matched donor, use of RIC and report of at least overall survival (OS) and/or progression-free survival (PFS) as endpoint. OS analyzed per intention to treat (ITT) was the primary endpoint. PFS, complete response $(\mathrm{CR})$ rate and rate of TRM were secondary endpoints.

Results: We identified 6 trials meeting entry criteria. There were 1192 patients assigned to TA and 630 patients to AR. Relative to TA, AR patients were more likely to experience TRM $(\mathrm{OR}=3.1,95 \% \mathrm{Cl}=2.1$ to 4.5$)$, but not more likely to achieve $\mathrm{CR}$ $(\mathrm{OR}=1.2,95 \% \mathrm{Cl}=0.9$ to 1.7$)$. There was no statistically significant difference in PFS within the first 36 months (HR=1.1, 95\% $\mathrm{Cl}=0.9$ to 1.3$)$ or beyond 36 months from transplant $(\mathrm{HR}=0.9$, $95 \% \mathrm{Cl}=0.7$ to 1.3 ). Similarly, there was no significant difference in OS between the arms within 36 months (HR=1.2, 95\% $\mathrm{Cl}=0.9-1.5)$ or beyond 36 months $(\mathrm{HR}=1.1,95 \% \mathrm{Cl}=0.7-1.6)$. Conclusion: We conclude that AR is associated with substantially higher TRM but with no improvement in CR rate, PFS or OS over TA transplantation in the initial management of patients with MM. Allogeneic transplantation in this setting remains experimental. Innovative approaches are needed to improve the safety and efficacy of this strategy.

\section{6}

Molecular remission after autologous-allogeneic tandem transplantation in patients with multiple myeloma

N. Kröger, T. Zabelina, F. Ayuk, C. Wolschke, H. Alchalby, A. Badbaran, D. Atanackovic, U. Bacher, T. Stübig, B. Fehse, A. Zander

University Medical Center Hambrug-Eppendorf (Hamburg, DE)

Introduction: In some patients (pat) with Multiple Myeloma (MM) long-term survival can be achieved after autologous (auto) or allogeneic (allo) stem cell transplantation (SCT). We prospectively investigated the incidence and impact of molecular remission (molCR) on long-term outcome after auto-allo tandem approach in MM (NCT 00781170).

Methods: From 4/2000 to $10 / 2008,73$ pat with median age of 50 years ( $r$ 29-64) and advanced stage II/III MM were included. Conditioning for auto SCT was melphalan $200 \mathrm{mg} / \mathrm{m}^{2}$ given over 2 days. After 2-3 months, dose-reduced conditioning with melphalan $140 \mathrm{mg} / \mathrm{m}^{2}$, fludarabine $180 \mathrm{mg} / \mathrm{m}^{2}$ and ATG Fresenius $®$ (Fresenius, Bad Homburg, Germany $10 \mathrm{mg} / \mathrm{kg}$ for related and $20 \mathrm{mg} / \mathrm{kg}$ for unrelated donors on day $-3,-2,-1$ ) followed by allo SCT was performed.
Remission was according to modified EBMT criteria. In 46 patients with $\mathrm{CR}$ or $\mathrm{nCR}$ minimal residual disease was monitored by patient-specific primers $(n=20)$ or highly sensitive plasma cell chimerism $(n=26)$. Sensitivity was $10-4$ to $10-5$ for plasma cell chimerism and 10-5 for nested PCR with patient-specific primers respectively.

Results: Overall 44 pat (60\%) achieved a CR according to EBMT criteria with negative immunofixation. $8 \%$ achieved a VGPR and $18 \%$ a partial remission. $3 \%$ had progressive disease and $11 \%$ were not evaluable for determination of remission. MolCR was observed in 30 pat, in 15 pat the molecular markers were sustained negative while in 15 pat molecular markers were only intermittently negative, resulting in an overall molCR rate of $46 \%$ and a stable sustained molCR rate of $23 \%$.

After a median follow-up at 7 years the 5 year progressivefree survival was $29 \%$ (95\% Cl: $17-41 \%)$. Patients without del $13 q 14$ had a significantly better PFS than those with del $13 q 14$ (5 y: $56 \%$ vs. $17 \%, p=0.02$ ). Patients who achieved $C R$ after transplantation had improved PFS in comparison to non-CR patients ( 5 y: $41 \%$ vs. $28 \%, p=0.008$ ). Patients with sustained negative molCR had a 5 year PFS of $85 \%$ vs. $31 \%$ for mixed molCR $(p=0.003)$

The 5 year overall survival was $52 \%$ (95\% Cl: $40-64 \%$ ). Patients without del $13 q 14$ had significantly improved 5 year survival ( $75 \%$ vs. $40 \%$. $p=0.02$ ). Patients who achieved a sustained molCR had a 5 year OS of $91 \%$ while those with mixed molCR resulted in a 5 year OS of $87 \%(p=0.06)$.

Conclusions: The study underlines the importance of the depth of remission and that achieving molCR is associated with longterm freedom from disease and potential cure of MM.

\section{Reduced-intensity Conditioning / Solid Tumours}

\section{7}

Reduced-intensity allogeneic stem cell transplantation for primary or post ET/PV myelofibrosis. Long-term follow-up of the prospective multicentre study of the Chronic Leukemia Working Party (CLWP) of the EBMT N. Kröger, H. Alchalby, T. Zabelina, D. Wolf, G. Kobbe, M. Bornhäuser, H. Baurmann, A. Nagler, W. Bethge, M. Stelljes, L. Uharek, K. Schaefer-Eckert, A. Buchert, P. Corradini, J. Schubert, M. Kaufmann, P. Dreger, G. Wulf, G. Stuhler, H. Kvasnicka, A. Zander, D. Niederwieser, R. Brand, T. de Witte on behalf of the Chronic Leukemia Working Party (CLWP) of the EBMT

Allogeneic stem cell transplantation (aSCT) is the only curative treatment for myelofibrosis. Here we present a long-term follow up of patients with myelofibrosis treated with reduced-intensity aSCT) in the prospective multicenter study conducted by the MDS subcommittee of the Chronic Leukemia Working Party of the EBMT (study registration NCT 00599547).

From 2002 to 2007, a total of 103 patients with primary (63 pts) or post-polycythemia vera and - essential thrombocythemia myelofibrosis (40 pts) from seventeen transplantation centers in three nations were included. There were 62 males and 41 females with a median age of 55 years (range, 32-68 years). Risk profile according to Lille score was low risk with constitutional symptoms $(17 \%)$, intermediate risk $(53 \%)$ and high risk $(30 \%)$. All but three of the patients received peripheral stem cells as stem cell source from either related $(n=33)$ or unrelated donor $(n=70)$ and a conditioning with Busulfan $(10 \mathrm{mg} / \mathrm{kg}$ orally or $8 \mathrm{mg} / \mathrm{kg}$ intravenously) ,Fludarabin $\left(180 \mathrm{mg} / \mathrm{m}^{2}\right)$ and ATG according a previously published protocol. After a median follow up of 60 months (range $9-109$ months), $45 \%$ of patients had a chronic graft vs. host disease which was extensive in the half of cases. The 5 years and 8 years estimated overall survival (OS) was $68 \%$ and $65 \%$, respectively with a stable 
plateau after 5,3 years follow up (Figure-1). Estimated 5-year disease-free survival was $40 \%$. The cumulative incidence of relapse/progression at 3 and 5 years was $22 \%$ and $28 \%$ and the non-relapse mortality at 1 and at 3 years was $18 \%$ ands $21 \%$, respectively.

In multivariate analysis advanced age $>55 y e a r s$ (HR: 4.69 , $p=0.001$ ), absence of JAK2V617F mutation (HR: 2.50, $p=0.02$ ), mismatched donor (HR: $3.62, p=0.002$ ) were significant independent predictors for reduced OS. During follow up relapse/ progression occurred in 28 patients. Twenty one of them were treated with donor-lymphocyte infusions (DLI) and/or a second allogeneic transplantation $(n=11)$. Fifteen of those were durably rescued with this treatment and were at the last follow up alive for a median interval of 49 months (range 25-61 months) after relapse/progression, whereas another one received recently a second transplantation for relapse and is still alive for a follow up of 4 months.

This update of a prospective trial using reduced intensity conditioning followed by allogeneic stem cell transplantation for myelofibrosis confirmed a very good long-term OS.

\section{8}

Allogeneic haematopoietic stem cell transplantation with reduced-intensity conditioning regimens in children and adolescents with very high-risk acute lymphoblastic leukaemia

V. Semenova, V. Stancheva, L. Alyanskiy, E. Ivanova, V. Babenko, N. Bondarenko, A. Slesarchuk, V. Paina, S. Zubarovskaya, V. Afanasyev

R.M. Gorbacheva Memorial Institute of Children Hematology and Transplantation, I.P.Pavlov SPMU (Saint-Petersburg, RU)

Aim: To compare efficacy of allo-HSCT with reduced intensity conditioning (RIC) and myeloablative conditioning (MAC) in children and adolescents with very high risk ALL (VHR ALL). Patients and Methods: 102 patients (pts) 1 to 21 (median 12) y.o. with VHR ALL underwent allo-HSCT between 2000 and 2010. VHR ALL was diagnosed due to late response to chemotherapy (induction failure), poor-risk cytogenetics ( $\mathrm{t}(9: 22)$, $t(4: 11))$, MRD $\left(>10^{-2}\right)$, infants with $11 \mathrm{q} 23$ rearrangement, short first remission, primary resistance or resistant relapse. AlloHSCT with RIC was performed in $31 \mathrm{pts}$ (RIC group) with Karnofsky (Lansky) $<80 \%$, history of therapy toxicity or ongoing infection: 20pts in 1st or 2nd complete remission (CR), 11 pts in resistant relapse. MAC was used in 71pts (MAC-group): 41pts in 1st or 2nd CR, 30pts were in more than 2nd CR or resistant relapse. RIC consisted of Flu $150 \mathrm{mg} / \mathrm{m}^{2}$ and $\mathrm{Mel} 140 \mathrm{mg} / \mathrm{m}^{2}$ (with or without ATG) or Flu $150 \mathrm{mg} / \mathrm{m}^{2}$ and Bu $8 \mathrm{mg} / \mathrm{kg}$ with or without ATG. MAC consisted of Bu $16 \mathrm{mg} / \mathrm{kg}$ (or Treo $36-48 \mathrm{mg} /$ $\mathrm{m}^{2}$ ) and Cph $120 \mathrm{mg} / \mathrm{kg}$ with or without ATG. Thirty pts received allo-HSCT from matched related donor (RIC, $n=7), 72$ pts from matched unrelated donor (RIC, $\mathrm{n}=24)$.

Results (Table 1): In RIC-group the pts engrafted on median D +18 (13-31). Patients with 1st or 2nd CR receiving allo-HSCT with RIC had the 7-years overall survival (OS) and event-free survival (EFS) of $51 \%$ and $36 \%, 6$ of these pts died within 100 days from HSCT (2 of infection and 4 of aGVHD). Six pts $(31 \%)$ relapsed, 5 of them achieved CR after chemotherapy and DLI or withdrawal of immunosuppression, 1 patient died of disease progression. Among patients undergoing alloHSCT with RIC in resistant relapse the CR was achieved in 5 of 11 pts. Nevertheless, the 1-year OS was $0 \%$. Three patients died of infection, 1 of aGVHD and 7 of progression. MAC group pts engrafted on median D+21 (10-49). In pts receiving allo-HSCT with MAC the 7 -years OS and EFS were $57 \%$ and $46 \%$. Ten $(26 \%)$ pts relapsed, but 2 of them achieved CR after chemotherapy and DLI, 9 pts died of relapse, 5 of aGVHD, 2 of infection, 1 from transplant-related toxicity, 1 due to engraftment failure. Among 30 pts in resistant relapse or more than 2nd CR receiving allo-HSCT with MAC 4 are currently in $\mathrm{CR}$, the other died from relapse (15), infection (7) or aGVHD (4).
Conclusion: Allo-HSCT with RIC is effective in young (<21 y.o.) patients with VHR ALL and its results are comparable with those of allo-HSCT with MAC.

\begin{tabular}{|l|c|c|c|}
\hline \multicolumn{1}{|c|}{ Parameters } & RIC n(\%) & MAC n(\%) & $p$ \\
\hline Total number & 31 & 71 & 0,25 \\
\hline $\begin{array}{l}\text { Non- } \\
\text { engraftment }\end{array}$ & $5(16 \%)$ & $6(8 \%)$ & 0,43 \\
\hline $\begin{array}{l}\text { OS, I or II CR- } \\
\text { group }\end{array}$ & $51 \%$ & $57 \%$ & 0,14 \\
\hline $\begin{array}{l}\text { EFS, I or II CR- } \\
\text { group }\end{array}$ & $36 \%$ & $46 \%$ & 0,07 \\
\hline $\begin{array}{l}\text { OS, relapse- } \\
\text { group }\end{array}$ & 0 & $18 \%$ & 0,47 \\
\hline $\begin{array}{l}\text { Relapse after } \\
\text { HCST, I or II } \\
\text { CR-group }\end{array}$ & $6(31 \%)$ & $10(26 \%)$ & \\
\hline
\end{tabular}

\section{9}

Treosulfan-fludarabine-ATG-F based reduced-toxicity conditioning regimen: multicentre "Allo-Treo" study, results in 183 patients with haematological malignancies A. Crotta (1), A. Lorusso (1), G. Martinelli (2), S. Cortelazzo (3), M.B. Pinazzi (4), G. La Nasa (5), R. Foà (6), S. Santarone (7), A. Rambaldi (8), A. Gallamini (9), R. Fanin (10), F. Merli (11), A.M. Carella (12), C. Corti (1), A. Ruggeri (1), M. Marcatti (1), M.T. Lupo Stanghellini (1), A. Assanelli (1), C. Messina (1), M. Bernardi (1), F. Ciceri (1), J. Peccatori (1)

(1)San Raffaele Scientific Institute (Milan, IT); (2)European Institute of Oncology (Milan, IT); (3)Ospedale San Maurizio (Bolzano, IT); (4)Ospedale San Camillo-Forlanini (Rome, IT); (5)P.O.R. Binaghi (Cagliari, IT); (6)Università La Sapienza (Rome, IT); (7)Ospedale Civile (Pescara, IT); (8)Ospedali Riuniti (Bergamo, IT); (9)Az. Ospedaliera Santa Croce e Carle (Cuneo, IT); (10)University Hospital (Udine, IT); (11)Arcispedale S. Maria Nuova (Reggio Emilia, IT); (12)IRCCS Casa Sollievo della Sofferenza (San Giovanni Rotondo, IT)

Allogeneic stem cell transplantation is the only curative option for many hematological malignancies, but toxicity of conditioning is still a limiting factor. Treosulfan is a bifunctional alkylating agent with reduced extra-hematologic toxicity profile. We evaluated the efficacy of Treosulfan-based conditioning regimen.

We analyzed 183 pts transplanted between 2004 and 2011 in 12 Italian centers. Median age 51 years (21-71), most common disease was acute leukemia (45\%), $50 \%$ of pts were transplanted in complete remission. Median Sorror score was 1 (0-7). Donor was unrelated (UD) in $57 \%$ of pts; source of stem cells was: BM $8 \%$, PBSC $81 \%$, CB $11 \%$ of pts. Conditioning: Treosulfan ( $14 \mathrm{~g} / \mathrm{msq}$ for 3 days) and Fludarabine $(30 \mathrm{mg} / \mathrm{msq}$ for 5 days). In vivo T and B-cell depletion was performed by ATG-Fresenius (10 mg/kg 3 days) and Rituximab (single $500 \mathrm{mg}$ dose) only in pts receiving an UD. GvHD prophylaxis: Cyclosporine $A$ and short course of Methotrexate, excepting CB. Median CD34+ infused for BM-PBSC was $6 \times 10^{6} / \mathrm{kg}(1-17)$, median TNC for CB was $3.2 \times 10^{7} / \mathrm{kg}$ (1.2-6; 11 pts received double $\left.\mathrm{CB}\right)$. Median follow-up was 35 months (3-84).

At day 60 cumulative incidence $(\mathrm{Cl})$ of neutrophil engraftment was $93 \pm 2 \%$, median time 17 days. In 164 evaluable pts molecular chimerism at day 60 was full-donor in $88 \%$. Median grade of regimen-related toxicity (CTC score) was $0(0-4)$, the most frequent toxicity was a transient rise of bilirubin (78\%). Grades II-IV aGvHD developed in 58 pts and 100 days-Cl was $28 \pm 3 \%$. Among 148 pts at risk, 54 developed chronic GvHD and 3 years$\mathrm{Cl}$ was $31 \pm 4 \%$, aGvHD (time dependent variable) increased the risk of $c G v H D(p=0.007)$. At 3 yrs $\mathrm{Cl}$ of relapse was $30 \pm 3 \%$ 
( $n=53$ ); no risk factors found. At 3 yrs DFS was $45 \pm 4 \%$, in multivariate analysis factors associated with a decreased DFS were: use of $\mathrm{CB}(\mathrm{HR}=2 ; \mathrm{p}=0.02)$, active disease at transplant $(\mathrm{HR}=2$, $p=0.001$ ). At 3 yrs $\mathrm{Cl}$ of TRM was $25 \pm 3 \%$; in multivariate analysis use of $C B(H R=2.9, p=0.005)$, active disease $(H R=2.7$, $p=0.002)$, aGvHD $(H R=3.5, p<0.001)$ were associated with increased TRM. Overall survival at 3 yrs was $50 \pm 4 \%$ and risk factors associated with decreased OS were: use of $\mathrm{CB}(\mathrm{HR}=2.8$, $p=0.001)$, active disease $(H R=2.3, p<0.001), a G v H D(H R=1.9$, $p=0.005)$, sorror $>0(H R=1.6, p=0.05) .86 p t s$ died after transplant, $40 \%$ for relapse and $60 \%$ for transplant related causes. Treosulfan is an effective myeloablative drug with low extrahaematological toxicities. Strategies to reduce aGvHD are warranted to deceased TRM.

\section{0}

Outcome of patients activating an unrelated donor search: the impact of transplant with reduced-intensity conditioning in a large cohort of consecutive high-risk patients

A. Rambaldi, B. Andrea, R. Fanin, F. Ciceri, F. Bonifazi, M. Falda, G. Lambertenghi-Deliliers, F. Benedetti, B. Bruno, P. Corradini, P. Alessandrino, P. lacopino, W. Arcese, R. Scimè, R. Raimondi, S. Sica, E. Todisco, T. Lamparelli, R. Oneto, L. Lombardini, S. Pollichieni, A. Algarotti, A. Carobbio, N. Sacchi, A. Bosi on behalf of the GITMO

Background: The clinical outcome of unrelated allogeneic hematopoietic stem cell transplantation (HSCT) using a reduced intensity conditioning (RIC) is often unsatisfactory because the transplant related mortality (TRM) and the relapse rate are still relevant. Most importantly, the lack of a suitable donor or other medical reasons often prevent transplantation in many patients for whom the search of an unrelated donor is activated.

Purpose: To compare the survival after unrelated HSCT with that obtained with any other therapeutic options in an unselected group of patients from the start of the unrelated donor search. Patients and Methods: We analyzed survival rates of a group of 326 consecutive, unselected patients for whom an unrelated donor search was activated between February 2002 and December 2004 by the Italian Bone Marrow Donor Registry, with the aim to perform an allogeneic RIC transplant. Only 2 conditioning regimens were allowed: program $\mathrm{A}$ was based on Melphalan $30 \mathrm{mg} / \mathrm{m}^{2}$, Alemtuzumab $80 \mathrm{mg}$, Fludarabine $90 \mathrm{mg} /$ $\mathrm{m}^{2}$ and Total Body Irradiation $200 \mathrm{cGy}$ (regimen $\mathrm{A}, \mathrm{n}=50$ ); program B was Thiotepa $10 \mathrm{mg} / \mathrm{kg}$, Cyclophosphamide $100 \mathrm{mg} / \mathrm{kg}$ and Antithymocyte Globulin $7.5 \mathrm{mg} / \mathrm{kg}$ (regimen $\mathrm{B}, \mathrm{n}=71$ ), followed by cyclosporine methotrexate. The selection of the conditioning regimen was the choice of the participating center. The outcome of patients grafted from an unrelated donor was compared to patients who did not find a donor, in a time dependent analysis, correcting for time to transplant. The median follow up from activation of donor search was 2.3 years.

Results: Unrelated donor transplant was associated with a significantly better survival in patients with acute leukemia $(\mathrm{HR}=0.63,95 \% \mathrm{Cl}=0.36-0.98, \mathrm{p}=0.047)$ and non Hodgkin's lymphoma $(\mathrm{NHL})(\mathrm{HR}=0.43,95 \% \mathrm{Cl}=0.23-0.78, \mathrm{p}=0.005)$ while only a favorable trend was documented for Hodgkin's Disease $(H D)(H R=0.65, p=0.114)$. No benefit on survival was registered for chronic leukemias. The outcome of the 2 different conditioning regimens was comparable, in terms of survival, transplant related mortality, and acute or chronic graft versus host disease.

Conclusions: Finding an unrelated donor and undergoing a RIC transplant significantly improves survival of patients with acute leukemia and NHL. The advantage is less clear for HD and chronic leukemias. The role of different conditioning regimens and GVHD prophylaxis with ATG or Alemtuzumab, remains to be elucidated by appropriate prospective clinical trials.
0261

Unrelated cord blood transplantation for children with high-risk or relapsed neuroblastoma

Y. Takahashi (1), K. Matsumoto (2), H. Fujisaki (3), F. Iwasaki (4), Y. Hashii (5), K. Nakamura (6), K. Sugita (7), H. Yabe (8), K. Kato (2), M. Takanashi (9), Y. Atsuta (10), M. Inoue (11)

(1)Nagoya University Graduate School of Medicine (Nagoya, JP); (2)Japanese Red Cross Nagoya First Hospital (Nagoya, JP); (3)Osaka City. General Hospital (Osaka, JP); (4)Kanagawa Children's Medical Center (Yokohama, JP); (5)Osaka University Graduate School of Medicine (Osaka, JP); (6)Hiroshima University Graduate School of Biomedical Sciences (Nagoya, JP): (7)University of Yamanashi (Yamanashi, JP); (8)Tokai University School of Medicine (Isehara, JP); (9)Japanese Red Cross Tokyo Blood Center (Tokyo, JP); (10)Nagoya University School of Medicine (Nagoya, JP); (11)Osaka Medical Center and Research Institute for Maternal and Child Health (Osaka, JP)

Background: Recent cooperative group studies for children with high-risk neuroblastoma (NB) report survival rates approaching $45 \%$ by multiple treatment modalities including surgery, radiation therapy and high-dose chemotherapy followed by autologous stem cell transplantation (SCT). Disease relapse remains the most common cause of treatment failure and the survival of patients with relapse or chemo-refractory disease has been reported less than $20 \%$. Recent advances in unrelated cord blood transplantation (UCBT) have provided increased chances for patients with hematological malignancies. Some case reports suggested graft versus NB effects after allogeneic SCT. However, only small series of UCBT for NB has been reported. We retrospectively analyzed the clinical outcome of UCBT for patients with NB in Japan.

Patients and Methods: We analyzed the clinical outcome of 75 children (47 boys and 28 girls) with NB (age, $<18$ years) who received UCBT in Japan, and registered to the Transplant Registry Unified Management Program (TRUMP). Overall survival (OS) and progression free survival (PFS) were analyzed using Kaplan-Meier estimates.

Results: The median age at transplantation was 3 years $(0-11$ years). Clinical stage of the patients was all stage IV (International Neuroblastoma Staging System) except for one stage III. Median follow-up period after UCBT was 401 days (from 8 to 4417 days). The probability of 3-year PFS and 3-year OS of the patients was $44.3 \% \pm 6.8 \%$ and $43.9 \% \pm 7.0 \%$ respectively. Of note, 3y PFS of the patients who achieved the 2nd complete response (CR) after relapse or the 1st partial response (PR: primary tumor has decreased by $50 \%$ to $90 \%$ decrease in the size of all measurable lesions) was $38.5 \% \pm 8.6 \%$, although this is significantly worse than that of the patients who obtained the 1st CR or very good partial response (VGPR: Primary tumor has decreased by $90 \%$ to $99 \%$, and no evidence of metastatic disease) $(51.5 \% \pm 9.5 \%, p=0.03)$. Causes of deaths after UCBT were relapse or progression of tumor $(n=22)$ and treatment related mortality $(n=13)$. Multivariate analysis for PFS or OS revealed that disease status at CBT (1st CR+VGPR), number of infused cells (over $4 \times 10 \mathrm{e} 7 / \mathrm{kg}$ patient's body weight) and GVHD prophylaxis (cyclosporine/tacrolimus+methotrexate) were significant better covariate factors.

Conclusion: These results suggest that UCBT could be the option for relapsed or chemotherapy resistant NB. Possible graft versus NB effects in UCBT should be further investigated. 


\section{2}

Allogeneic stem cell transplantation for advanced metastatic renal cell cancer: the experience of two prospective French multicentre trials

R. Devillier (1), G. Gravis (1), J.O. Bay (2), M. Michallet (3), J.Y. Cahn (4), E. Deconninck (5), R. Tabrizzi (6), J.H. Bourhis (7), S. François (8), C. Faucher (1), M. Mohty (1), D. Blaise (1) (1)Institut Paoli Calmettes (Marseille, FR); (2)Centre Jean Perrein (Clermont-Ferrand, FR); (3)Hôpital Edouard Herriot (Lyon, FR); (4)CHU Michallon (Grenoble, FR); (5)Hôpital Jean Minjoz (Besançon, FR); (6)Hôpital Haut Lévêque (Bordeaux, FR); (7)Institut Gustave Roussy (Villejuif, FR); (8)Centre Hospitalier Régional (Anger, FR)

Patients with metastatic renal cell cancer (RCC) have poor outcome. However, immunotherapies as Interleukine 2 (IL-2) and/ or Interferon alpha (INF-a) have been reported to induce remission and improve survival. This particular immunosensitive feature of RCC opened perspective for the use of allogeneic stem cell transplantation (Allo-SCT). Remissions and prolonged survivals after Allo-SCT for advanced metastatic RCC have been previously reported suggesting a potential of Graft-versus-tumor (GVT) effect. We report here the French experience in this setting and evaluated the response rate after Allo-SCT. A total of 47 patients with relapsed and/or refractory metastatic RCC were included from 2001 to 2005 in 2 prospective trials (TS-Allo n=31; GETUG-11 n=16) and underwent reduced intensity conditioning (RIC) Allo-SCT from a matched related donor. Median age at Allo-SCT was 51 [34-68]. At inclusion, median number of previous treatment line for metastatic disease was 2 [1-4], performance status (PS) was $\leq 1$ for 31 patients, and 34 patients $(72 \%)$ presented with progressive disease (PD). RIC regimens were Fludarabine, Busulfan and Antithymocyte globulin in the TS-Allo protocol and Fludarabine Cyclophosphamide and low dose (2 Gy) total body irradiation in the GETUG-11 protocol. Ten patients (21\%) (7 with PD at Allo-SCT) achieved a partial response (PR) and $15(44 \%)$ of the 34 patients in PD at Allo-SCT achieved stable disease (SD). PR and SD occurred in a median time of 35 days [11-262] after Allo-SCT. Nineteen of 22 patients $(86 \%)$ with PR or SD presented a novel progression after a median time of 4.6 months [1.1-19.3]. Four patients died from non relapse cause: 1 died early at day 30 and 3 died in PR from graft-versus-host disease at day 62, 105 and 244. Cumulative incidence of grade 2-4 acute at day 100 and chronic GVHD at 6 months were $55 \%$ and $44 \%$ respectively. One-year overall (OS) survival was $29 \%$ [95Cl: 19-47]. No factors (proto$\mathrm{col}$, number lines of previous treatments, PS or disease status at Allo-SCT) were found to be associated with either response or OS. Finally, despite an encouraging response rate of $46 \%$ in these patients with advanced refractory metastatic RCC, outcome remains poor with short response duration and no long term survivor. However, the recent development of antiangiogenic therapies remains unsatisfactory. Allo-SCT to induce response associated with these agents to prolong response duration deserves to be study in selected population.

\section{3}

Sites of relapse in patients with metastatic soft tissue sarcoma who have undergone allogeneic SCT. Report of the CWS-Group and the German-Austrian SCT Registry E. Koscielniak (1), P. Bader (2), P. Lang (3), E. Hallmen (1), R. Handgretinger (3), S. Bielack (1), U. Gross-Wieltsch (1), U. Thiel (4), R. Ladenstein (5), T. Klingebiel (2)

(1)Klinikum Stuttgart, Olgahospital (Stuttgart, DE); (2)University Children's Hospital (Frankfurt, DE); (3)University Children's Hospital (Tübingen, DE); (4)University Children`s Hospital (Munich, DE); (5)St.Anna Children`s Hospital (Vienna, AT)

Objective: The prognosis of patients with STS who present or relapsed with distant metastases has not improved despite intensification of chemotherapy with or without stem cells rescue. The use of allo-SCT has been initiated for these patients under the assumption of the graft versus sarcoma effect (GvT). In Germany there are two ongoing prospective trials (Frankfurt and Tuebingen) on the role of haploidentical SCT in patients with STS. Additionally, many centers performed allo-SCT in high risk STS patients outside prospective studies. Allo-SCT is regarded mainly as immunotherapy exerting its activity against residual disease. There is, therefore, still controversy over what impact on prognosis an aggressive local therapy (LT) to all metastatic sites, if feasible would have for the outcome after allo-SCT and what would be the optimal time for LT.

Methods and Results: To answer this question we have analysed sites of relapse after allo- SCT in 61 patients with primary (18) or secondary (43) metastatic STS registered in the CWS Studies and SCT registry between 1990 and 2010. 43 pts were $>10 \mathrm{yrs}$ of age. Different busulfan and melphalan based conditioning regimen were used. 18 patients were treated in prospective trials in Frankfurt or Tuebingen. 27 allografts were received from MRD, 30 from MMRD, 2 from MUD and 2 from MMUD. 29 pts were in CR or VGPR the time point of SCT, other had stable (28) or progressive (4) macroscopic tumour manifestation (PR). 18 out of 29 pts who were in CR/VGPR at SCT relapsed in a median time of 8 months (range 2-41). 8/29 patients died in CR of therapy related reasons (TRD), 6 maintained their CR for a median time of 42 months (5-136) after SCT. Only 2 patients relapsed in previous tumor sites (primary tumor or metastases), 5 revealed mixed relapses in old and new localisations, 10 patients developed new metastatic sites (soft tissue, bone, abdomen). Local therapy consisted in the majority of patients of RTX with or without surgery. 8 out of 28 patients with PR prior to SCT achieved CR at day 100 after SCT. 3 died of TRD, 4 relapsed subsequently mainly in old tumour localisation.

Conclusion: Only patients with limited tumour burden might benefit from GvT. The tumour spread after SCT suggest that local control of macroscopic manifestations is a prerequisite but microscopic dissemination seems to be the main obstacle for cure. Additional post-transplant immunotherapeutic approaches are needed to better control the disease.

\section{Stem Cell Research and Experimental Stem Cell Transplantation}

\section{4}

Bone and haematopoietic niche formation is predetermined by a specific epigenetic signature

A. Reinisch (1), N. Etchart (1), N.A. Hofmann (1), M. Frühwirth (1), D. Thaler (1), B. Feilhauer (1), A. Ortner (1), C. BehamSchmid (1), S. Trajanoski (1), W. Wagner (2), K. Schallmoser (1), D. Strunk (1)

(1)Medical University of Graz (Graz, AT); (2)WTH Aachen University Medical School (Aachen, DE)

Human mesenchymal stem and progenitor cells (MSPCs) from various tissues are evaluated in clinical trials for bone and marrow regeneration. Better understanding MSPC functionality in vivo could help to select optimal cells for specific disorders thereby increasing clinical efficiency. Here we demonstrate that epigenetic predisposition of bone marrow (BM) MSPCs facilitates cartilage, bone and hematopoietic microenvironment formation in vivo.

BM, white adipose tissue (WAT), umbilical cord (UC) skeletal precursors and skin fibroblasts were subjected to surface immune phenotyping before adipo-, chondro- and osteogenic differentiation in vitro. In vivo mesenchymal tissue formation was tested by transplanting MSPCs subcutaneously into NSG mice. Sequential cartilage, bone and BM formation was evaluated by in vivo imaging and histology. Infiltration of host hematopoiesis was studied by analyzing lin-/sca1+/c-kit+ (LSK) cells using polychromatic flow cytometry. Epigenetic profiling comparing bone forming with non bone-forming MPSCs was 
done using a whole genome methylation array. Translation of epigenetic regulation into gene transcription was evaluated by qRT-PCR.

MSPC from all sources show an almost identical immunephenotype. Efficient chondrogenic differentiation of BM MSPCs can distinguish this source in vitro. Transplantation studies in vivo suggest that only BM MSPCs are capable to form cartilage and bone through endochondral ossification thereby generating a niche for hematopoietic invasion. Comparing methylation profiles, BM MSPCs show a unique signature and cluster separately. Most differentially methylated genes include transcription factors critically involved in endochondral ossification. $\mathrm{CpG}$ island hypomethylation results in increased transcription into mRNA.

$\mathrm{BM}$ is the only tissue containing precursors with true endochondral differentiation potential. This is reflected by a BM-specific epigenetic signature predisposing these cells to form cartilage and bone. In addition this developmental process is critically involved in hematopoietic niche formation. Our data suggests a more critical evaluation of in vivo differentiation capacity mainly supporting the use of BM-derived MSPCs for bone and marrow regeneration.

\section{5}

Early in vivo signalling signature during human stem/ progenitor cell-derived neo-vasculogenesis R. Rohban (1), N. Etchart (1), A. Reinisch (1), N. Hofmann (1), D. Thaler(1), B. Feilhauer(1), M. Dacheva (1), K. Schallmoser(1), E. Rohde (2), D. Strunk (1)

(1)Medical University of Graz (Graz, AT); (2)Paracelsus Medical University of Salzburg (Salzburg, AT)

Introduction: It has previously been shown by us and others that stable therapeutic human neo-vasculogenesis in vivo depends on co-transplantation of pericytes or their mesenchymal stem/ progenitor cells (MSPCs) with endothelial cells or endothelia colony-forming progenitor cells (ECFCs) (Reinisch et al., Blood, 2009). Here we describe a novel strategy for unraveling the mediators of neo-vasculogenesis by proteomic profiling of the early signaling signature in vivo providing us with tools to develop strategies for therapeutic intervention as well as regenerative applications.

Methods: MSPC and ECFCs were transplanted subcutaneously in matrigel plugs alone or at a ratio of 20:80 into immune deficient NSG mice. Implants were harvested $24 \mathrm{~h}$ after transplantation for proteomic profiling using KAM 1.3 antibody microarray (www.kinexus.ca) testing over 800 signaling and phospho-proteins. The state of vessel formation and stability of the transplants were verified by histological follow-up of corresponding explants for 2 and 8 weeks after transplantation. Therapeutic targets were selected from antibody microarray based on differential display and were used for in vitro toxicity and viability assays as well as in vivo modulation of therapeutic vasculogenesis.

Results and Discussion: Results confirmed that co-transplantation of ECFCs with MSPCs was most efficient for forming stable perfused human vessels. "ECFC only" plugs showed vessel formation after transplantation of higher cell number and later in the time course after transplantation. Early chondrogenesis was observed in "MSPC only" plugs after 8 weeks.

Protein microarray data analysis revealed significant alteration of components including (1) caspases, DAXX and P53 involved in death-associated pathways, (2) ERB, MAPK, mTOR and TGF- $B$ signalling, (3) Focal adhesion, VEGF, JAK-STAT and Wnt signaling. Selected targets are currently validated by in vitro and in vivo blocking experiments.

Conclusion: Transplantation of more than one purified cell type is needed for tissue engineering and vascular regeneration in vivo. Proteomic profiling unraveled independent and partially overlapping signaling networks involved in the complex process of vascular regeneration.

\section{6}

Ex vivo generation of erythroid cells from human induced pluripotent stem cells of different origin

I. Dorn (1), P. Schlenke (1), M. Radstaak (2), W. Sibrowski (1), H.R Schöler (2), H. Zaehres (2)

(1)University of Münster (Münster, DE); (2)Max Planck Institute for Molecular Biomedicine (Münster, DE)

Objectives: The manufacturing of red blood cells (RBC) from human induced pluripotent stem cells (iPS) offers the potential to generate large quantities of patient's specific RBC from an unlimited source. However, in vitro recapitulation of physiological erythropoiesis from embryonic pluripotent stem cells (ESC), which includes mesoderm induction, generation of hematopoietic stem cells (HSC), followed by erythroid maturation, hemoglobin switching and enucleation, remains a challenge.

Methods: We used human ESC (H1) and iPS cells from human fibroblasts, neuronal stem cells and CD34+ HSC for the ex vivo generation of erythroid cells. First, ESC/iPS cells were allowed to form embryoid bodies (EB) under cytokine stimulation for $>15$ days. Thereafter, EB were dissociated and single cells were applied to a three-step protocol for human erythropoiesis over 18-25 days. Hematopoietic maturation was analysed by colony formation, flow cytometry (CD34, CD45, CD36, glycophorin A (GPA)), microscopy after May-Grünwald-Giemsa staining and hemoglobin analysis.

Results: The used EB protocol allowed for the generation of $3 \%-15 \%$ CD34+ and CD45+ cells from human ESC/iPS cells, able to form erythroid and myeloid colonies in semisolid cultures. These hematopoietic cells further developed into erythroid precursors as determined by $>80 \%$ expression of CD36 and GPA, followed by maturation into normoblasts and partially enucleated RBC. Manufactured cells mainly contained embryonic and fetal hemoglobin. Compared to ESC, first results indicate a lower proliferation capacity of iPS cells, whereas maturation into RBC was partially higher.

Conclusions: We were able recapitulate the multistep development of RBC from human ESC and iPS cells of different origin. This might contribute as a first step towards the large-scale ex vivo generation of patient's specific RBC from human iPS cells for transfusion purposes. Furthermore, the iPS related hematopoiesis and erythropoiesis might serve as a model for studying various hematopoietic diseases.

\section{7}

Platelet-derived factors preserve human mesenchymal cell stemness and promote endochondral bone and marrow niche formation via PDGF-receptor signalling N. Etchart, A. Reinisch, A. Ortner, M. Frühwirth, N. Hofmann, R. Rohban, C. Url, K. Schallmoser, C. Beham-Schmid, D. Strunk Medical University of Graz (Graz, AT)

Mesenchymal stem/progenitor cells (MSPC) are defined by their capacity to differentiate into bone, cartilage and fat cells in vitro. However, their limited engraftment potential in vivo led to the assumption that the transient therapeutic benefits observed after MSPC transplantation are predominantly due to paracrine effects. Based on preliminary observations indicating superior functionality of MSPC expanded under the aegis of human platelet lysate (HPL), we hypothesised that the lack of MSPC engraftment and differentiation in vivo can be reverted by human platelet-derived factors.

We compared human bone marrow MSPC expanded in HPLsupplemented culture medium to MSPC derived in foetal bovine serum (FBS). Both cell types can differentiate into osteo-, adipo- and chondrocytes in vitro. However, 3D chondrogenesis in vitro of HPL-MSPC creates $>3$ times heavier cartilage fragments containing more hypertrophic chondrocytes than those generated with FBS-MSPC, suggesting that chondrogenesis is favoured by human platelet-derived factors.

In a new model of spontaneous bone formation in vivo, HPLMSPC from all 17 donors form bone through an endochondral 
mechanism after inclusion in Matrigel ${ }^{\mathrm{TM}}$ and subcutaneous implantation in immune-deficient NSG mice, without requiring osteogenic pre-induction. Remarkably, $>60 \%$ of these human ossicles attract mouse bone marrow, indicating that HPL-MSPC can establish a bone marrow-supporting niche. In contrast, only $20 \%$ of FBS-MSPC lines are able to form bone.

Detailed phenotypic analysis reveals that the stem cell marker SSEA-4 is expressed at significantly higher levels on HPLMSPC than on FBS-MSPC, suggesting that MSPC stemness is maintained by humanised culture. Higher SSEA-4 expression of human MSPC in vitro also exquisitely correlates with attraction of mouse haematopoiesis in vivo. In addition, HPL-MSPC could be re-isolated and re-expanded to large numbers from mouse marrow-populated implants and formed bone again in secondary human ossicles, suggesting that stemness is also maintained in vivo.

To elucidate the underlying mechanism, HPL-MSPC were treated in vitro with Imatinib, which inhibits PDGF-R gamma phosphorylation. This results in a drop of SSEA-4 surface expression similar to that observed in FBS cultures and in a loss of cartilage and bone differentiation potential in vivo.

Our data indicate that PDGF-R gamma signalling is chiefly implicated in the maintenance of human MSPC stemness as well as bone and marrow niche forming competence during humanised expansion.

\section{8}

Regulatory role of umbilical cord mesenchymal stem cells on Treg and Th17 cells in patients with systemic lupus erythematosus

D. Wang, H. Liu, L. Lu, X. Li, L. Sun

The Affiliated Drum Tower Hospital of Nanjing University Medical School (Nanjing, CN)

Objective: To investigate the regulatory effect of umbilical cord mesenchymal stem cells (UCMSC) on regulatory T cells (Treg) and Th17 cells in patients with systemic lupus erythematosus (SLE).

Methods: Human UCMSC were isolated, expanded and infused into 15 refractory SLE patients (NCT00698191, phase II). Clinical efficacy was evaluated. The percentages of Treg and Th17 cells in peripheral blood were detected by flow cytometry. Peripheral cytokines were determined by ELISA and real time PCR, respectively. Peripheral blood mononuclear cells (PBMC) from 11 active SLE were co-cultured with UCMSC to examine the changes of Treg and Th17 cells and supernatant cytokines. Patients: naturally occurring Treg (nTreg) were isolated from peripheral blood and co-cultured with UCMSC in vitro in the presence of anti CD3/28 microbeads and recombinant IL-2. Results: Significant clinical efficacy was found by UCMSC transplantation. Peripheral blood CD4+CD25+Foxp3+T cells percentage showed obvious increase at 1 week $(2.32 \% \pm 1.40 \%)$, 1 month $(2.03 \% \pm 1.30 \%), 3$ months $(2.41 \% \pm 2.06 \%)$ and 6 months $(2.81 \% \pm 0.69 \%$ ) visits ( all $p<0.05$ vs pre-MSCT $1.58 \% \pm 1.02 \%)$, in parallel with significant decline of CD3+CD8-IL17A+T cells after MSCT $(1.30 \% \pm 1.02 \%$ at 1 week, $0.73 \% \pm 0.60 \%$ at 1 month, $0.56 \% \pm 0.60 \%$ at 3 months, $0.52 \% \pm 0.40 \%$ at 6 months, all $p<0.05$ vs pre-MSCT $1.69 \% \pm 1.13 \%$ ). Real time PCR showed that gene expression of RORgammat decreased, while TGFbeta1 increased significantly 1 week after transplantation. Plasma concentration of TNFalpha and IL17A decreased while TGFbeta1 increased. The co-culture of UCMSC with SLE PBMC resulted in a statistical increase of CD4+CD25+Foxp3+T cells and reduce of CD3+CD8-IL17A+T cells (all $p<0.05$ at UCMSC: PBMC 1:1, 1:10, 1:50 ratio), but without a dose dependent manner. Supernatant TGFbeta1 and IL6 increased but TNFalpha decreased significantly in the co-culture system. The addition of TGFbeta1 or IL6 inhibitor to the culture system statistically abrogated the upregulation of Treg, while had no effect on Th17 cells. The absolute number of nTreg in the presence of UCMSC increased compared to that of nTreg alone, and showed significant enhanced expression of Foxp3 and GITR. Additionally,
CFSE dilution suggested that UC-MSC can obviously promote the proliferation of SLE nTreg in vitro.

Conclusions: UCMSC transplantation markedly regulated Treg and Th17 cells in SLE patients, which may be one of the mechanisms for its therapeutic potential in refractory SLE.

\section{9}

Interferon-gamma is essential for allogeneic FoxP3+ regulatory $\mathrm{T}$ cell-mediated GvHD-suppression

C. Koenecke, C.-W. Lee, L. Föhse, A. Ganser, R. Förster, I. Prinz

Hannover Medical School (Hannover, DE)

Objective: In experimental acute graft-versus-host disease (GvHD), adoptive transfer of natural FoxP3+ regulatory T cells (Tregs) has proven its potential to prevent a lethal outcome of alloreactive attack. However, it is so far not clear by which mechanism Tregs achieve this goal. Here we address the role of interferon-gamma for donor Treg-mediated GvHDsuppression.

Methods: We used blocking anti-interferon-gamma mAb and interferon-gamma-deficient (Ifng-l-) donor mice in Treg cotransplantation studies in a C57BL/6 into BALB/c mouse model of acute GvHD. GvHD severity was monitored by survival, clinical score and histological analysis.

Results: We found that Tregs expressing the transcription factor FoxP3 produced large amounts of interferon-gamma during acute GVHD. Intracellular staining identified alloreactive donor Tregs to be a major source of interferon-gamma after transplantation. In fact, more than $50 \%$ of allogeneic but not residual endogenous FoxP3+ Tregs produced interferon-gamma. Notably, blocking of Interferon-gamma with specific mAb led to death from GVHD. Furthermore, only wild type Tregs, but not Tregs from Ifng-/- donor mice could prevent fatal GVHD suggesting that Treg-intrinsic interferon-gamma production was required for their protective function.

Conclusion: Our data imply that interferon-gamma, in particular in highly inflammatory Th1 cytokine dominated environments such as acute GVHD, can be produced by natural Tregs and has essential immune-regulatory functions.

\section{0}

Studying HLA-restricted allo-reactivity in vivo using mice reconstituted with human antigen-presenting cells

M. Sommer, S. Klobuch, D. Wolff, S. Thomas, M. Theobald, W. Herr, R. Meyer

University Medical Center of the Johannes GutenbergUniversity (Mainz, DE)

Persisting antigen-presenting cells (APC) play a pivotal role as stimulators for donor-derived T cells in acute Graft versus host disease (GvHD). Strategies to attenuate GvHD by targeting host-APC (e.g. antibodies) are often species-specific and preclinical models are lacking. It has been demonstrated by others that functional human $\mathrm{B}$ cells can be established in mice reconstituted with human hematopoietic stem cells (HSC). Here, we focused on the establishment of further APC populations in mice reconstituted with human HSC

HSC were injected into sub-lethally irradiated NOD-scid gamma-/- (NSG) mice. Blood and skin samples were taken fortnightly. After 6 weeks, animals were sacrificed for obtaining bone marrow (BM) and spleen. Reconstitution of human APC was surveyed for up to 36 weeks by using different cohorts. The engraftment rate in 13 independent experiments was $70 \%$. B cells, NK cells, monocytes, and HLA DR+/CD11c+ myeloid dendritic cells (DC) in BM and spleen were detected after 6 weeks. After 12 weeks, plasmacytoid DC were also present in BM. T cells were not found before week 18. We stained skin biopsies for the presence of human CD1a / CD207-positive Langerhans cells (LC) as surrogates for tissue-resident DC. Human LC were present in murine epidermis beyond week 6 . 
In an attempt to test whether the engrafted human APC function as targets for allo-reactive $T$ cells, we generated naïve CD8-positive T-cell lines from partially HLA-matched third party donor by mixed lymphocyte cultures. In first experiments, these T cells $\left(10^{7}\right.$ cells/animal) were injected into mice that had been reconstituted with human HSC previously. Following transfer, the established human hematopoiesis was partially depleted. In mice 8 weeks after HSC-transfer, only B cells were reduced. In contrast, in mice 14 weeks after HSC-transfer, mDCs and monocytes were also depleted. These preliminary findings indicate that beyond human B cells, monocytes as well as mDCs in NSG-mice engrafted with human HSC might function as targets for allo-reactive $T$ cells.

In summary, human APC engraft NSG mice and the engraftment of DC is not restricted to primary hematopoietic organs, as shown for epidermal LC. Allo-reactive CD8 T-cells deplete human APC in vivo which indicates their functionality as T-cell targets. Still, $\mathrm{mDC}$ and monocytes seem to develop their functionality later than B cells. To improve our mode, factors accelerating APC-development (e.g. FLT3) are currently under investigation.

\section{Late Complications}

\section{1}

Incidence, mortality and biological characteristics of Bronchiolitis obliterans after allogeneic haematopoietic stem cell transplantation

M. Ditschkowski, T. Gromke, A. Elmaagacli, R. Trenschel, N. Steckel, M. Koldehoff, D. Beelen

University hospital of Essen (Essen, DE)

Bronchiolitis obliterans (BO) after HSCT is a terrifying lateonset non-infectious pulmonary complication (LONIPC) leading to increased morbidity and mortality. Until now very limited therapeutic approaches are available and clinical course depends on diagnosis at an early stage.

Of 1779 patients (pts) who underwent allo-HSCT at our center between January 2000 and December 201068 pts (39 male, 29 female; median age at transplant 45 years) were diagnosed $\mathrm{BO}$ at a median onset of 18 (range 7-178) months after HSCT. In this cohort 18 pts received grafts from HLA-identical siblings (26\%), 32 pts from matched ( $47 \%$ ) and 18 pts from mismatched $(26 \%)$ unrelated donors. Transplants consisted of unmanipulated peripheral blood stem cells $(n=63 ; 93 \%)$ or bone marrow $(n=5 ; 7 \%)$. Based on pulmonary function impairment BO was characterized "severe" in 29 (43\%), "moderate" in 29 (43\%) and "mild" in $10(15 \%)$ pts. Concomitant chronic GVHD was observed in 65 (96\%) BO pts. The calculated 5-year cumulative incidence of BO was $6.8 \%$. A total of 18 BO pts (26\%) died corresponding to a calculated 5 -year mortality of $22 \%$. In the BO cohort pts younger than 30 years of age at HSCT and pts with a smoking history showed significantly decreased 5 -year survival $(p=0,05$ and $p=0,039)$. A significantly higher proportion of $\mathrm{BO}$ pts $(69 \%)$ compared to non-BO HSCT recipients $(51 \%)$ was transplanted from $\mathrm{ABO}$ incompatible donors. BO pts demonstrated a markedly transient dip of CD4+CD25+ T cells of median $35 \%$ of the previous levels at the time of BO onset. Blood quantitative measures of the hypoxia-inducible factor 1 (HIF-1a) expression were significantly higher in $\mathrm{BO}$ patients compared to healthy controls $(p=0,02)$ or chronic GVHD pts without lung involvement $(p=0,007)$. Serum immunoglobulin $G$ (IgG) levels were significantly decreased at onset of $B O$ compared to $\operatorname{lgG}$ values of HSCT recipients without BO at the corresponding post-transplant time points $(5,5 \mathrm{~g} / \mathrm{l} v \mathrm{v} .9,1 \mathrm{~g} / \mathrm{l}$, $p=0,033)$. The median exhaled NO concentrations were lower in BO pts than in HSCT pts without BO. By using a threshold level of $15 \mathrm{ppb}$ of exhaled NO a significant discrimination between $B O$ and non-BO pts $(p=0,009)$ was enabled.
Our data demonstrate that BO is a serious LONIPC with substantial mortality. Certain cellular and molecular blood markers and proteins but also breath gas analyses might be useful for an early diagnosis.

\section{2}

Mortality in 2-year survivors of autologous HSCT in

Australia: findings from the Cancer after Stem Cell Transplantation (CAST) study

R. Thielbeer (1), C. Vajdic (2), T. O'Brien (3), A. Dodds (4), L. Wilcox (5), L. Ashton (1)

(1)Children's Cancer Institute Australia (Randwick, AU); (2)Lowy Cancer Research Centre (Randwick, AU); (3)Sydney Children's Hospital (Randwick, AU); (4)St Vincent's Hospital (Darlinghurst, $A U$ ); (5)ABMTRR (Darlinghurst, $A U$ )

Background: In Australia, autologous haematopoietic stem cell transplantation (HSCT) is used to treat a growing number of patients with cancer each year, particularly patients of older age. While long-term survival has been studied extensively in allogeneic HSCT recipients, few studies have examined longterm survival in autologous HSCT recipients.

Aim: To evaluate rates of mortality in 2-year survivors of autologous HSCT.

Method: Over 13000 HSCT recipients treated from 1992-2007 in Australia were assembled for the CAST study. Deaths following HSCT were identified from the Australasian Bone Marrow Transplant Recipient Registry and through data linkage with the National Death Index. Overall survival estimates and standardised mortality ratios (SMRs) were calculated for 4547 patients aged 15 or older who survived at least 2 years after autologous HSCT.

Results: By the end of 2007, 1466 deaths had occurred among 2 -year survivors of autologous HSCT. Approximately $70 \%$ of deaths were due to relapse or progression of disease. Relapse remained the most common cause of death beyond 10 years from HSCT. Overall, the survival probability was $56 \%$ at 10 years from HSCT. 10-year survival probabilities by diagnosis ranged from $34 \%$ for patients with multiple myeloma to $90 \%$ for patients with testicular cancer. In the interval 2 to 5 years from HSCT, observed mortality was more than 17 times higher than expected based on rates in the age- and sex-matched Australian general population $(S M R=17.9,95 \% \mathrm{Cl}=16.9-19.0)$. While observed mortality moved closer to expected mortality in the intervals 6 to 10 years $(S M R=10.2,95 \% \mathrm{Cl}=9.1-11.5)$ and 11 or more years from $\mathrm{HSCT}(\mathrm{SMR}=5.9,95 \% \mathrm{Cl}=4.2-8.4)$, observed mortality remained significantly increased across all periods examined. For all diagnoses, rates of observed mortality were significantly greater than expected in the general population. SMRs by diagnosis ranged from $7.4(95 \% \mathrm{Cl}=5.6-9.7)$ for patients with acute myeloid leukaemia to $35.9(95 \% \mathrm{Cl}=32.8-43.0)$ for patients with breast cancer.

Conclusion: Mortality in 2-year survivors of autologous HSCT is significantly increased compared to the Australian general population, even beyond 10 years from HSCT. With the majority of deaths occurring from relapse, prevention of disease recurrence is the greatest challenge for autologous HSCT recipients.

\section{3}

Long-term survival and quality of life assessment after allogeneic stem cell transplantation for haematological malignancies; comparable results following myeloablative and reduced-intensity conditioning

A. Shimoni, E. Tallis, N. Shem-Tov, Y. Volchek, R. Yerushalmi, A. Nagler

Chaim Sheba Medical Center (Tel-Hashomer, IL)

SCT is associated with substantial mortality during the first 2 years after SCT whereas afterwards survival often reaches plateau. QoL is increasingly recognized as an important longterm end-point. The pattern of late events and QoL has been 
reported following myeloablative conditioning (MAC) but is not well defined in reduced-intensity (RIC) setting. To explore late outcomes we retrospectively analyzed SCT results among 726 patients (pts) transplanted between $1 / 2000$ and 8/2009. 246 pts were alive and disease-free 2 years after SCT. Median age was 51 years (17-72). Diagnoses included AML/MDS $(n=131)$, $\operatorname{ALL}(n=24)$, lymphatic diseases $(n=48), C M L / M P D(n=29)$, nonmalignant $(n=14)$. Conditioning was MAC $(n=72)$, RIC $(n=118)$ or reduced-toxicity myeloablative conditioning (RTC, $n=56$ ). 172 pts had cGVHD, graded moderate-severe (mod-sev) in 44 and $29 \%$ of pts after MAC and RIC/RTC, respectively $(p=0.03)$. 68 and $43 \%$ were still on immune suppressive therapy (IST) 2 years after SCT, respectively $(p=0.001)$. With median followup of 68 months after SCT (25-140), the probability of pts surviving disease-free 2 years after SCT to remain disease-free for the next 5 years was 84 and $82 \%$ after MAC and RIC/RTC, respectively $(p=N S)$. There were 35 deaths beyond 2 years, 15 due to relapse and 20 due to non-relapse causes (NRM). The cumulative incidence of NRM was $7 \%$ after both MAC and RIC/RTC. The cumulative incidence of relapse was $9 \%$ and $11 \%$, respectively $(p=N S)$. Age $>55$ and mod-sev cGVHD predicted shorter survival, HR $2.1(p=0.07)$ and $2.6(p=0.006)$, respectively. Mod-sev cGVHD predicted NRM, HR $5.2(p=0.001)$. Advanced disease predicted relapse, HR $2.6(p=0.004)$. The probability of stopping IST by 8 years was 59 and $75 \%$ after MAC and RIC/RTC, respectively $(p=0.001)$. Median IST duration was 30 and 20 months, respectively $(p=0.05)$. QOL was assessed by the EORTC QLQ-C30 questionnaire. Mean QoL score was 69,66 and 65 after MAC, RIC and RTC, respectively. Low QoL score was reported by 15,14 and $19 \%$, respectively $(p=N S)$. Continuous need for IST and depression correlated with a low score while healthy lifestyle (return to work, physical and sexual activity) and academic education correlated with high scores. In conclusion, late outcome is similar after MAC and RIC/RTC. Late NRM is similar although cGVHD is less severe and IST duration shorter after RIC/RTC. Pts who are disease-free 2 years after SCT, particularly younger pts with no mod-sev cGVHD can expect good survival and QoL.

\section{4}

Long-term complications and quality of life after reducedintensity conditioning allogeneic stem cell transplantation A. Clavert (1), Z. Peric (1), X. Cahu (2), P. Chevallier (1), E. Brissot (1), F. Malard (1), T. Guillaume (1), J. Delaunay (1), S. Ayari (1), V. Dubruille (1), S. Le Gouill (1), B. Mahe (1), T. Gastinne (1), N. Blin (1), J.-L. Harousseau (3), P. Moreau (1), N. Milpied (4), M. Mohty (1)

(1)Hematology (Nantes, FR); (2)Hematology (Rennes, FR); (3)Hematology (Saint Herblain, FR); (4)Hematology (Bordeaux, FR)

Background: RIC allo-SCT is increasingly used in elderly or frail patients (pts) not eligible for myeloablative conditioning. While the natural history of LTC and QOL are rather well described in the myeloablative allo-SCT setting, data is still sparse in the RIC setting. This single centre survey analyzed the outcome and features of LTC and QOL in 110 RIC allo-SCT pts who survived for a minimum of 2 years (y) after allo-SCT. QOL was assessed in a cross-sectional study using the EORTC QLQC30 questionnaire and the FACT-BMT questionnaire.

Results: The K-M estimate of overall survival (OS) was 93\% $(95 \% \mathrm{Cl}, 88-99 \%)$ and $81 \%(95 \% \mathrm{Cl}, 71-94 \%)$ at 5 and $10 \mathrm{y}$, respectively. The primary cause of death was relapse found in 4 pts $(4 \%)$. Six pts $(5 \%)$ died of non-relapse-related causes as secondary malignancies $(n=3)$, chronic GVHD (cGVHD; $n=2)$ and infection $(n=1)$.

With a median follow-up of 4.6 y (range, 2-12.1), cGVHD was the most prevalent LTC with a cumulative incidence (CInc) of $66 \%(95 \% \mathrm{Cl}, 57-74)$ at $10 \mathrm{y}$. In multivariate analysis, a mismatched unrelated donor allo-SCT was the strongest independent risk factor associated with the development of $L T C(R R=4.06$, $95 \% \mathrm{Cl} 1.81-9.10, \mathrm{p}=0.002)$.
In this series, 61 pts (55\%) responded to the different questionnaires. Overall, in the EORTC QLQ-C30 questionnaire 70\% of these pts reported a good to very good QOL, with a mean global QOL group score of 71.7 (SD, 20.8). Compared to the group without cGVHD, pts with cGVHD had significantly lower QOL. Similarly, in the FACT-BMT questionnaire the scores in all subscales indicated good QOL. However, there was statistically significant differences between the two groups in average QOL scores in the physical and functional well-being and BMT subscales, as well as for the FACT-G, FACT-TOI and FACT-BMT total composite scores. Moreover, the QOL survey revealed that $29 \%$ of working age-patients could return to full-time work. Finally, 2 childbearing age women (18\%) were reported to have had pregnancies.

Conclusion: Pts who are alive beyond 2 y after RIC allo-SCT have a high probability of cure with excellent OS and good QOL. However, cGVHD remains an issue requiring long-term appropriate psychological support. Also, the natural history of LTC after RIC appears to be different from that described in the standard myeloablative setting, warranting more research in this field and lifelong surveillance through a close partnership between the transplant center and organ-specific specialities.

\section{5}

Fludarabine-based reduced-intensity or reduced-toxicity conditioning may be associated with increased risk for secondary malignancies after allogeneic stem cell transplantation

A. Shimoni, E. Tallis, N. Shem-Tov, Y. Volchek, R. Yerushalmi, A. Nagler

Chaim Sheba Medical Center (Tel-Hashomer, IL)

Secondary malignancies are a known complication in longterm survivors after SCT. The incidence has been reported mostly after myeloablative conditioning (MAC). Fludarabine (F)-based RIC and RTC are associated with lower incidence of early SCT-related complications, however due to the so far relatively limited long-term follow-up, the incidence and riskfactors for secondary malignancies following these regimens is not well defined. We retrospectively reviewed a single institution database of 931 allogeneic SCTs given over 12 years to identify patients (pts) with secondary malignancies. Conditioning regimens included standard MAC $(n=257$, TBI-based in 111), F-based RIC [ $n=449$, including $F$ with intermediate-dose busulfan (FB2) and $F$ with melphalan (FM)] or F-based RTC $[n=225$, including $F$ with high-dose busulfan (FB4) and $F$ with treosulfan (FT)]. 21 pts had secondary malignancies including squamous cell carcinoma of the skin $(n=5)$, penis $(n=1)$ vagina $(n=1)$, tongue $(n=1)$ and esophagus $(n=2)$, colon cancer $(n=3)$, breast cancer $(n=2)$, pancreatic cancer $(n=2)$, metastatic cancer of unknown primary $(n=1)$, melanoma $(n=1)$, metastatic sarcoma $(n=1)$, Kaposi sarcoma $(n=1)$. Median age at SCT was 53 years (29-70). 19 pts were given F-based RIC/RTC and none had TBI. The median time from SCT was 49 months (7-138). 17 pts had prior chronic GVHD, 10 moderate-severe, 14 were still on immune-suppression at diagnosis of secondary malignancy. The 10 -year cumulative incidence was $5.3 \%(95 \% \mathrm{Cl}$, $3.2-8.7 \%$ ). It was $2.2 \%, 8.8 \%, 5.5 \% 7.8 \%$ and $5.5 \%$ after BuCy, FB2, FB4, FM and FT respectively ( $p=0.05$ for BuCy Vs. RIC/ RTC). Multivariate analysis identified RIC/RTC and moderatesevere chronic GVHD as adverse prognostic factors with HR $5.9(p=0.03)$ and $2.7(p=0.04)$, respectively. Pts were treated with surgery for localized tumors and with chemo-radiotherapy or palliative therapy for metastatic disease. Currently, 17 pts are alive and 4 have died, all of them had advanced solid tumor at presentation. 10-year cumulative incidence of death due to secondary malignancy was $1.6 \%$. In conclusion, secondary malignancies are rare but significant complication after allogeneic SCT. Curative approach is feasible in a subset of pts. The combination of $\mathrm{F}$ and intermediate to high-dose alkylating agents may be associated with higher risk for second malignancies than standard therapy with high-dose alkylator and/or 


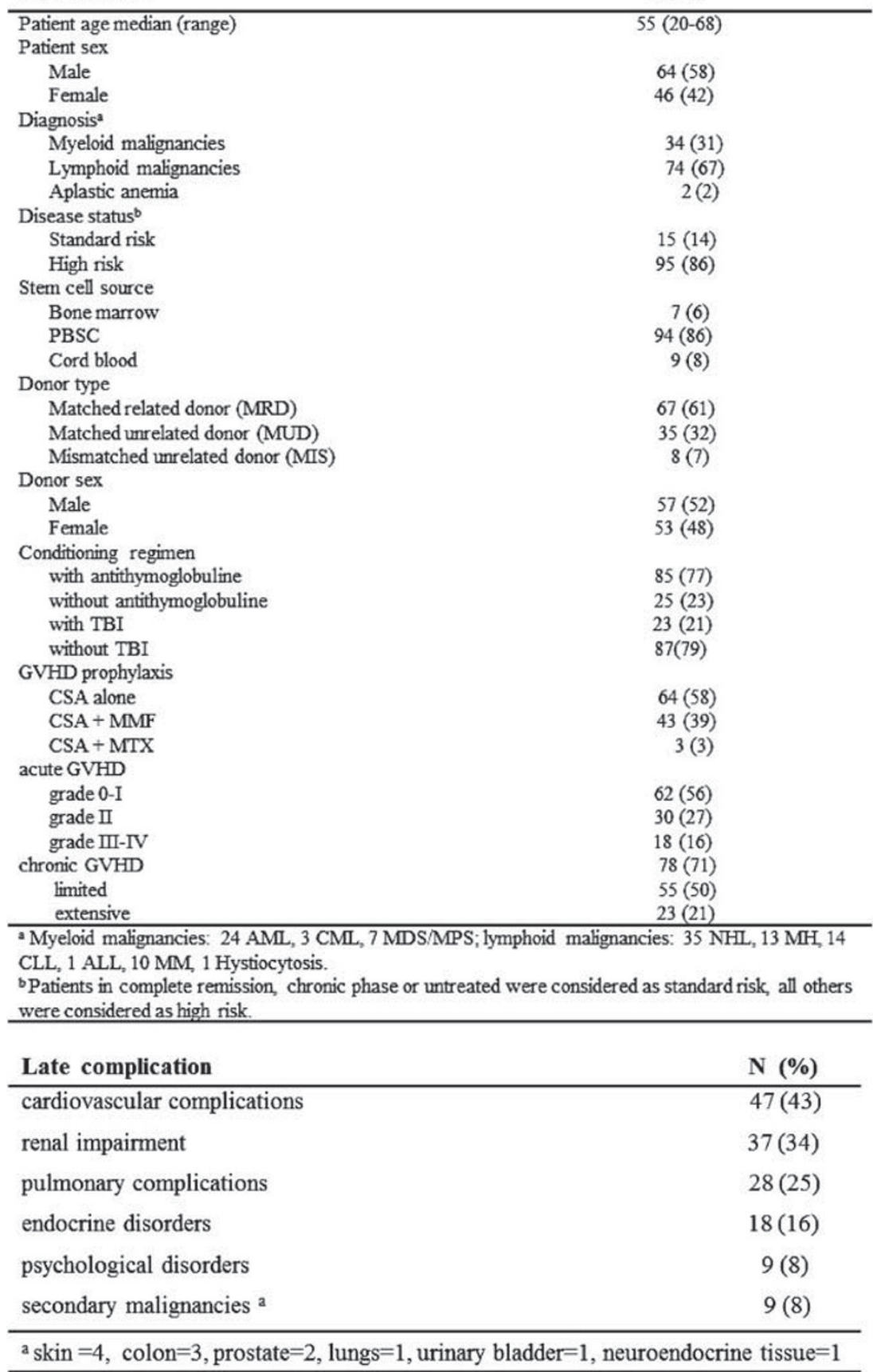

TBI. Larger studies with a larger number of events are needed to confirm these observations.

\section{6}

Genetic background of metabolic syndrome in children treated with stem cell transplantation

S. Skoczen, M. Bik-Multanowski, J. Gozdzik, K. Dolezal, A. Dluzniewska, A. Krasowska-Kwiecien, W. Czogala, O. Wiecha, J.J. Pietrzyk, J. Starzyk, M. Siedlar Jagiellonian University (Krakow, PL)

Background: Metabolic syndrome (MS) is diagnosed in up to $23 \%$ of patients treated with haematopoietic stem cell trans- plantation (HSCT) and $30-40 \%$ of children with obesity. The purpose of the study was to determine the genetic background of the syndrome in children treated with HSCT.

Patients and Methods: Thirty patients (pts.) 1,5-19,4 (average 9,9 ) years old, 24 boys and 6 girls, diagnosed with ALL-15, ID5, AA-4, AML-4, MDS-1, NBL-1 treated with HSCT (autologous1) according to EBMT protocols were included to the study. The blood was collected before and 7 months in average after HSCT (19 pts.). The control group was composed of 29 children 3-18 (average 13) years old, 15 boys and 14 girls with obesity. There were no signs and symptoms of the primary disease in children after HSCT, all of them completed immunosuppressive treatment. BMI before HSCT was 13,3-27,4 (average 19,1), BMI percentile 0,1-98,3 (average 56,2). BMI after HSCT was 
9,7-28 (average 18,7), BMI percentile 0,1-87,8 (average 51,3). BMI in the obesity group was $21,8-38,4$ (average 31,3 ), BMI percentile 94,2-100 (average 98). Blood samples (0.5 ml) were drawn from all the study participants. Subsequently, total RNA extraction was performed and microarray analysis was conducted with GeneChip Human Gene 1.0 ST Arrays (Affymetrix) according to the manufacturer's protocol. We decided to compare the patient without overweight treated with HSCT (low risk of activation of genes responsible for MS development) with children with obesity (high risk of activation of genes responsible for MS development).

Results: BMI percentile $<85$ was noticed in 20 and 15 patients before and after HSCT respectively. Among 200 significantly differentially expressed genes when comparing children before HSCT with $\mathrm{BMl}<85$ with control group, 108 were underexpressed and 92 overexpressed in the control group. Only higher than 1.5 (Fold Change) differences in gene expression were analyzed. Among others, the IGFBP2 gene was underexpressed in the control group. This gene encodes IGF-binding protein, which inhibits IGF-mediated growth and developmental rates. This relationship was not observed after HSCT.

Conclusions: As there is now substantial evidence linking IGFBP-2 with nutritional status and insulin sensitivity, changes in expression of IGFBP2 gene in patients after HSCT may be involved in development of metabolic syndrome after HSCT. Study sponsored by national grant number K/PBW/000520.

\section{7}

Sustained increased risk of cardiovascular disease in the second decade post-transplant in male long-term survivors of allogeneic haematopoietic stem cell transplantation

J. Barrett, P. Pophali, M. Battiwalla

National Heart, Lung, and Blood Institute (Bethesda, US)

Long term survivors of SCT are at increased risk from cardiovascular (CV) disease. We evaluated CV risk in 109 recipients (62 males, 47 females) surviving $>5 \mathrm{yr}$ after SCT. Ninety-nine patients received $\geq 12$ Gy TBI conditioning and 10 received non-myeloablative conditioning followed by bone marrow (15) or T cell depleted peripheral blood SCT (94). SCT indications were CML (56), acute leukemia (30), MDS (13), and others (10). Median ages at SCT, 5 and $10 \mathrm{yr}$ follow-up were 34,40 and 46 yrs, respectively. Ninety-four individuals developed cGVHD and 36 required immunosuppressive treatment for $>3 \mathrm{yrs}$. Survivors were followed up at $5,7,10,15 \mathrm{yrs}$ postSCT and had $>90 \%$ Karnofsky performance status at the time of analysis. PostSCT $\mathrm{CV}$ events were uncommon: 2 survivors had percutaneous coronary interventions and 1 had recurrent pulmonary emboli. Serial electrocardiograms revealed 6 new ischemic changes and 28 new rhythm/conduction abnormalities. Serial echocardiograms showed no change from baseline. At 5 and 10 yrs postSCT respectively, $44 \%$ and $52 \%$ of survivors had abnormal lipid profiles. Despite being younger, $23 \%$ of survivors met the Adult Treatment Panel III threshold for dyslipidemia treatment at $5 \mathrm{yrs}$ post SCT, which is higher than the $15 \%$ at $45-54 \mathrm{yrs}$ in the general population (Multi-Ethnic Study of Atherosclerosis). There were significant increases in prevalence of hypertension $(p<0.001)$, diabetes $(p=0.018)$, elevations in $C$-reactive protein $(C R P)$ $(p<0.001)$ and body mass index $(p=0.044)$ postSCT compared to baseline. The Framingham general $\mathrm{CV}$ risk score of males at 5 yrs postSCT predicted a $10 \mathrm{yr}$ risk of CV events - double the expected norm (median $10.4 \%$ vs. 5.4\%). The calculated median heart/vascular age was $8 \mathrm{yrs}$ ahead of patients' chronological age. Females received hormone replacement therapy postSCT and none had increased CV risk scores. To explore CV risk factors, we examined presence and persistence of cGVHD beyond 3 yrs and CRP as an inflammatory marker. cGVHD was not associated with $\mathrm{CV}$ risk at any time point but CRP correlated significantly with the $\mathrm{CV}$ risk at 5 yrs postSCT $(p=0.02)$. All risk factors stabilized between 5 and $10 \mathrm{yrs}$ postSCT. These studies highlight a significant increased risk of $\mathrm{CV}$ events in male SCT recipients in their second and third decade after SCT.Identifying mechanisms underlying the inflammatory processes associated with CV risk would be a first step in developing effective preventative management of heart disease in SCT recipients.

\section{Increase in cardiovascular risk factors post SCT}

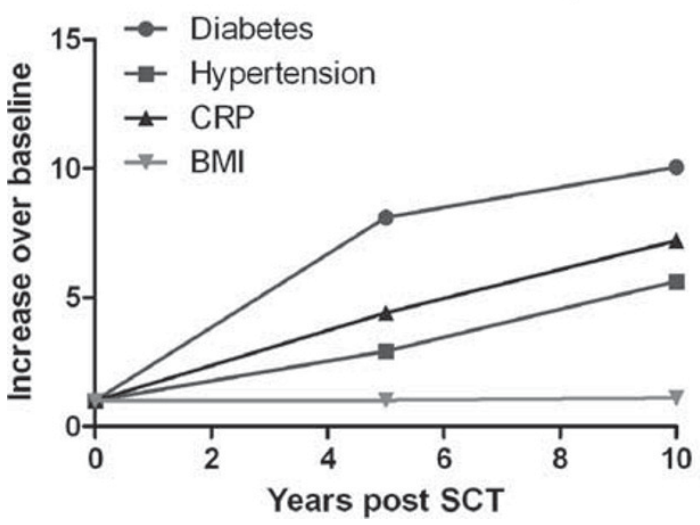

\section{Cellular, Gene Therapies and Cytokines}

\section{8}

Mesenchymal stromal cells for irradiation-induced gastro-intestinal lesions: first experiences in four over-irradiated patients

J. Voswinkel (1), N. Gorin (1), J. Simon (2), J.J. Lataillade (3), M. Benderitter (4), P. Gourmelon (4), L. Fouillard (1), S. Francois (5), A. Chapel (4)

(1)Saint Antoine Hospital APHP (Paris, FR); (2)Pitie-Salpetriere University Hospital (Paris, FR); (3)Percy Military Hospital (Clamart, FR); (4)Radiological Protection and Human Health Division, Institute of Radiological Protection and Nuclear Safety (Fontenay-aux-Roses, FR); (5)UMRS-938, UPMC University (Paris, FR)

Background: Four patients under radiotherapy for prostate cancer were accidentally overexposed to irradiation which led to severe therapy-resistant diarrheal and painful hemorrhagic colitis and recto-vesical/recto-prostatic fistula. Mitigation of lethal intestinal injury, following high doses of irradiation, can be achieved by injection of Mesenchymal Stromal Cells (MSC). MSC accelerate functional recovery of the intestine and dampen the systemic inflammatory response by reducing the levels of proinflammatory cytokines in radiation-induced gastrointestinal syndrome.

Objective: To provide compassionate therapy for refractory irradiation-induced colitis and diminish pain, haemorrhage and diarrhea, allogeneic bone marrow (BM)-derived MSC from family donors were intravenously infused to four patients.

Material: MSC were obtained by culture from BM aspirates of their children. Three patients received $5 \times 10^{6} / \mathrm{kg}, 2.6 \times 10^{6} / \mathrm{kg}$ and $5 \times 10^{6} / \mathrm{kg}$ MSC, respectively, by intravenous infusion. Before, one month and six months after MSC therapy the clinical parameters pain, haemorrhage and fistulisation (MRI, colonoscopy) were evaluated. One patient was retreated $\left(0.8 \times 10^{6} / \mathrm{kg}\right.$ of MSC) after 6 months. Recently a fourth patient was treated by two MSC injections $\left(1.85 \times 10^{6} / \mathrm{kg}\right.$ and $2.4 \times 10^{6} / \mathrm{kg}$ one week later). In this patient, lymphocyte subsets and frequency of diarrhea were monitored.

Results: No toxicity occurred. After MSC therapy two patients revealed a substantiated clinical response for pain and hemorrhage. In one patient pain reappeared after 6 months and again substantially responded on a second MSC infusion (Figure 1). In one patient a beginning fistulisation process could be 
stopped. In this case a stable remission has been observed for more than 3 years of follow-up. The fourth patient underwent two injections of MSC: The frequency of painful diarrhea diminished from 5-7 per day to 3 per day after the first MSC injection and stabilized at 2-3 per day after the second injection. In parallel, after the MSC injections, an increase of NK cells and B lymphocytes and a decline of CD4+ and CD8+ T lymphocytes was observed (Figure 2). Prostate cancer remained in stable complete remission in all patients.

Conclusion: In four patients with refractory irradiation-induced colitis systemic MSC therapy was safe and effective on pain, diarrhea, haemorrhage and inflammation. MSC may represent a safe therapeutic measure for patients under radiotherapy overirradiated.

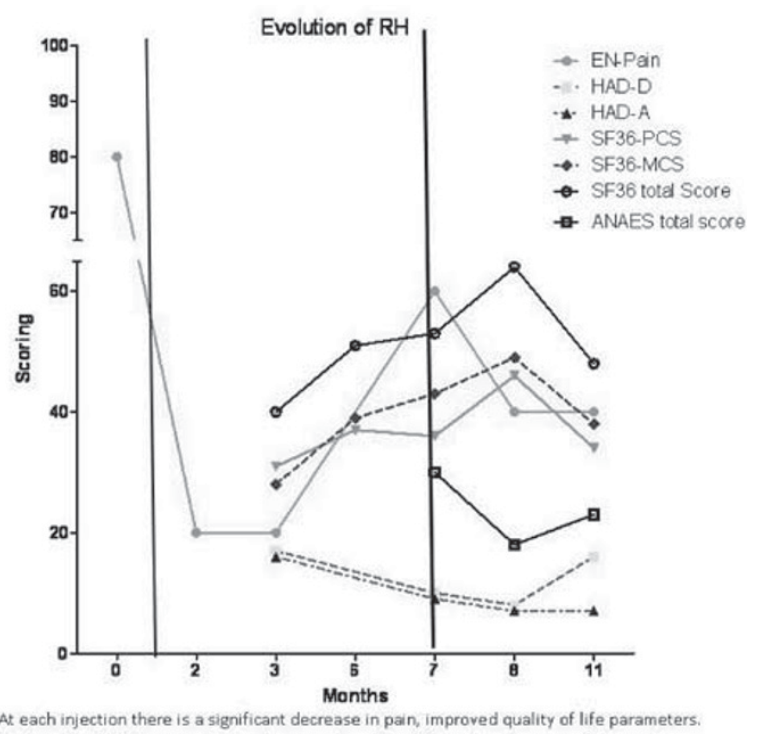
At 4 months (M4) increased pain, decreased quality of life and an increase of depression

[0278]
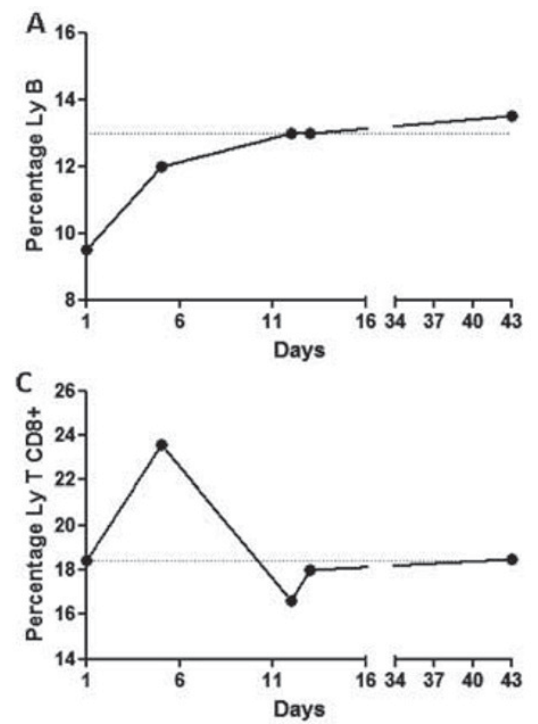

A. The peroentage of total B cells appears to increase from the first day and the peroentage appears to increase a function of time. During the 43 days of observations, there is a progressive increase of over $40 \%$ of LV B cells, the peak of increase of LY B is detected during the first week, with an increase of $25 \%$. B: The percentage of $\mathrm{Ly} T \mathrm{CD} 4+$ cells appears to decrease progressively and slightly during the first 43 days, there was a decrease of $16 \%$.

C. The peroentage of $\operatorname{CD} 8+T$ cell increases very rapid y during the first 5 days (28\%) and then decreases to return to its initial value.

D: No variation in the percentage of NK cells during the first 12 days, then was a marked increase in this cell population. In the 43 th day we detect a $50 \%$ increase compared to the 12 th day.
0279

O27 the treatment of steroid resistant GvHD: clinical results and biological readouts in a multicentre study

G. Lucchini (1), E Dander (1), F Pavan (1), A Balduzzi (1), S Bonanomi (1), P Perseghin (2), I Di Celie (1), G. Gaipa (1),

$D$ Belotti (1), M. Parma (2), M. Introna (3), A. Rambaldi (3), A. Biondi (1), A. Rovelli (1), G. D`Amico (1), E. Biagi (1)

(1)Università Milano Bicocca (Monza, IT); (2)Ospedale San Gerardo (Monza, IT); (3)Ospedali Riuniti di Bergamo (Bergamo, IT)

Objective: Mesenchymal stromal cells (MSC) are multipotent cells with broad immunomodulatory properties. Their role in the treatment of graft versus host disease (GvHD) has been intensively investigated. The present study reports the outcome of a cohort of patients (pts) receiving MSCs as part of GvHD treatment.

Othods: Pts exhibiting acute or chronic grade II-IV steroidresistant GvHD after allogeneic stem cells transplantation were

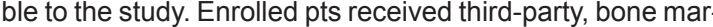
row derived, platelet lysate-expanded MSCs for a minimum of 3 doses. Median dose was $1 \times 10^{6} / \mathrm{cells}$ per $\mathrm{kg}$ body weight. The suggested infusion interval was 5-7 days. Clinical response to treatment was assessed 28 days after the last MSC infusion. GvHD was graded according to NIH criteria. Before MSC infusion and weekly till day +28 after the last MSC infusion, blood samples were collected to analyse lymphocyte subsets and GvHD activity biomarkers.

Results: From August 2009 to September 201123 pts (13 adults, 10 pediatric) aged 1-58 years received MSCs. GvHD 列 single organ in 12 pts and multiple organs in 11 cases. GvHD was defined as grade II in 7 pts, grade III in 12 and grade IV in 4 pts. In this series overall response to MSC infusion was $60,8 \%$, and complete response was $17,3 \%$. None of the pts affected by chronic GvHD benefited from the treatment. Both skin and gut GvHD presented a good response rate, skin showing an earlier response (2-4 days) compared to gut (5-7 days). Pts with multiple organ involvement and higher GvHD grade seemed to show a worse response to treatment. 12 out of 23
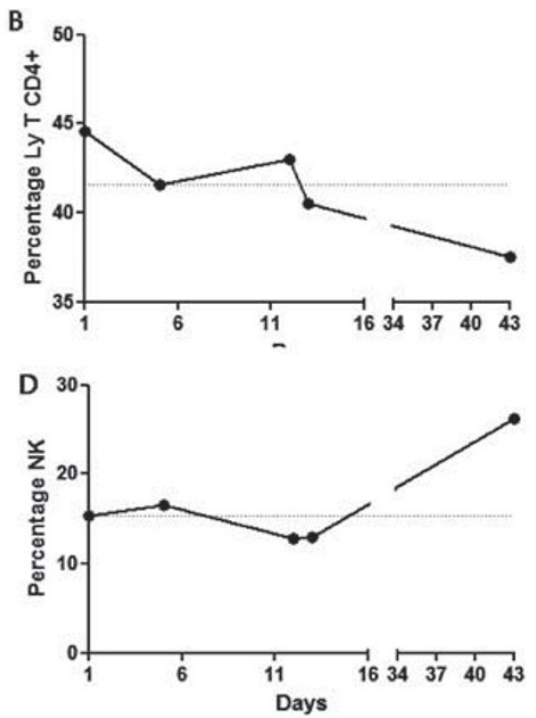
treated pts are alive; 5 pts died from GvHD complications, 3 pts died from infectious events, and 2 relapsed. Immunobiological data support the idea that MSC are able, in responding pts, to increase $T$ regulatory cells, as demonstrated by the progressively decreasing in Th1/Treg andTh17/Treg ratio. Biomarkers of GvHD activity correlated with MSC response.

Conclusions: The present study underlines the safety of PLexpanded MSC use in children and adults. MSC efficacy seems to be greater as early as the administration is performed, in acute rather than in chronic GvHD, as well as in milder from of the disease involving skin and gut, particularly in pediatric pts. Biological readouts corroborate the clinical observations and hint at the ability of MSCs to increase T regulatory cells as mechanism of action against GvHD.

\section{0}

Administration of cord blood (CB)-derived virus-specific cytotoxic T lymphocytes to prevent CMV, adenovirus, and EBV infection after CB transplant

P. Hanley (1), C. Martinez (1), J. Melenhorst (1), K. Leung (1), B. Savoldo (1), A. Leen (1), G. Dotti (1), A. Gee (1), C. Rooney (1), H. Heslop (1), R. Krance (1), A. Barrett (2), E. Shpall (3), C. Bollard (1)

(1)Baylor College of Medicine (Houston, US); (2)NHLBI, National Institutes of Health (Bethesda, US); (3)M.D. Anderson Cancer Center (Houston, US)

CMV, Adenovirus (Ad) and EBV are viral pathogens causing morbidity and mortality in patients after HSCT and cord blood transplantation (CBT). We have shown that adoptive immunotherapy with peripheral blood (PB) donor derived multivirusspecific Cytotoxic $T$ Lymphocytes (mCTLs) directed against EBV, CMV and Ad can effectively prevent and treat the clinical manifestations of these viruses after HSCT. We have now extended these studies by expanding $\mathrm{MCTL}$ from $\mathrm{CB}$ to restore cellular immunity to CMV, EBV and Ad simultaneously after CBT. The development of $\mathrm{mCTLs}$ for patients undergoing CBT requires the priming and extensive expansion of naîve $T$ cells rather than the more limited and simple direct expansion of pre-existing memory $T$ cell populations from virus-experienced donors. We have developed a novel protocol utilizing an initial round of stimulation with autologous CB-derived dendritic cells transduced with a recombinant Ad5f35 vector carrying a transgene for the immunodominant CMV antigen, pp65 (Ad5f35pp65) in the presence of IL-7, IL-12 and IL-15. This is followed by 2 rounds of weekly stimulation with autologous Ad5f35pp65transduced EBV-LCL in the presence of IL-15 or IL-2.

So far we have enrolled 7 patients and treated 4 patients on this phase I study where the CTLs were generated from the $20 \%$ fraction of a fractionated CB unit. All 7 patients engrafted neutrophils and platelets $<30$ and $<60$ days post CBT respectively. CTL were infused to 4 patients from 63-146 days post CBT at doses of $5 \times 10 \mathrm{e} 6 / \mathrm{m}^{2}$ to $1 \times 10 \mathrm{e} 7 / \mathrm{m}^{2}$. No infusion-related toxicities or GvHD was observed. Within 2 weeks of CTL, all patients had detectable EBV-specific T cells in their PB that persisted $>1$ year post CTL. Three patients remain free of CMV, EBV, and Ad reactivation up to 1 year post CBT. One patient was transiently viremic for Ad and CMV but after 2 doses of CTL permanently cleared the CMV and Ad from the PB and stool respectively. He remains asymptomatic and virus free $>1$ year post-CBT. Analysis of this patient's PB showed a concomitant rise in CMV and Ad-specific T cells by 4 weeks. Further, using TCR deep sequencing we have shown that $\mathrm{T}$ cell clones present in the CTL lines of all patients, but not in their PB pre infusion, expand up to 14 fold post infusion. In summary, administration of low doses of CB derived virus-specific CTL to patients after CBT has so far been safe and can facilitate reconstitution of virusspecific T cells and control viral reactivation/infection.

\section{1}

Outcomes of CD19-directed multivirus specific cytotoxic T lymphocyte therapy for patients with relapsed B cell malignancies after allogeneic haematopoietic stem cell transplantation

C. Bollard (1), C. Cruz (1), B. Savoldo (1), R. Krance (1), R. Kamble (1), A. Barrett (2), C. Hosing (3), E. Shpall (3), H. Heslop (1), A. Leen (1), C. Rooney (1), M. Brenner (1), G. Dotti (1) (1)Baylor College of Medicine (Houston, US); (2)NHLBI, National Institutes of Health (Bethesda, US); (3)M.D. Anderson Cancer Center (Houston, US)

Allogeneic hematopoietic stem cell transplant (HSCT) may increase long term disease-free survival in patients with Bcell malignancies, but delayed immune reconstitution associated with the procedure is associated with viral infections and disease relapse. We hypothesized that a single T-cell platform mediating both antiviral and antileukemic activity could benefit these patients. We prepared CTL with specificities through native receptors directed towards EBV/CMV/adenovirus (Ad), then engineered them to express a chimeric antigen receptor (CAR) targeting CD19. We used donor-derived antigen presenting cells expressing Ad antigens and transgenic CMVpp65 following transduction with the Ad vector Ad5f35CMVpp65. Multivirus (MV)-specific CTL were then transduced with a retroviral vector encoding CAR-CD19.28zeta. Safety: 6 patients were infused with 1.5 to $4.5 \times 10 \mathrm{e} 7 \mathrm{cells} / \mathrm{m} 2$ without infusion related toxicity. One CLL patient developed fever, diarrhea, hypotension 4 weeks post CTL. Findings were consistent with ileitis at a known site of disease. Biopsy of the gut showed an absence of normal and malignant $B$ cells, and the presence of CARCD19.28z T cells. Persistence: There was a predictable decline of $\mathrm{T}$ cells in peripheral blood (PB) following infusion. However, persistence is documented by their presence in disease sites (GIT and bone marrow) up to 9 weeks. Anti-viral activity: Patient 1 had Ad positivity in stool, which resolved without antiviral treatment. No other patient developed viral infections post CTL. Anti-tumor activity: Of the two patients with $\mathrm{Ph}+\mathrm{ALL}$, one patient had $4 \%$ blasts detectable in the PB at the time of CTL which cleared within 2 weeks post CTL. She subsequently relapsed and then died of disease 7 months post CTL. The other patient had a transient decrease in blast count to $0.2 \%$ but ultimately progressed after 4 weeks. 4 CLL patients have been treated. The first CLL patient had resolution of lymphadenopathy within 2 weeks but following the disappearance of CTLs from PB, progressed and died after 2 months. The second CLL patient has had stable disease for over 6 months with an influx of T-cells in his bone marrow. The remaining 2 CLL patients are still early but by 6 weeks both have had reductions in their CLL counts without toxicity. These results provide encouraging evidence of the safety, persistence, and effectiveness of monoculture CD19CAR multivirus T cells in the treatment of high risk B-cell malignancies post allogeneic HSCT.

\section{2}

Sequential administration of DLI and cytokine-induced killer cells in patients relapsing after allogeneic haematopoietic stem cell transplantation: preliminary results from a phase II study

M. Introna (1), A. Algarotti (1), C. Micò (1), E. Todisco (1), A. Pievani (1), G. Borleri (1), J. Golay (1), A. Grassi (1), A. Salvi (1), I. Cavattoni (2), S. Deola (2), E. Biagi (3), A. Biondi (3), S. Cortelazzo (2), A. Rambaldi (1)

(1)Ospedali Riuniti (Bergamo, IT); (2)Ospedale Regionale Generale (Bolzano, IT); (3)Cinica Pediatrica Università di Milano Bicocca (Monza, IT)

Background: Cytokine Induced Killer (CIK) cells show non restricted, NK-like anti-tumoral cytotoxicity in vitro and in vivo, with little graft-versus host disease (GVHD). CIK cells can be produced under clinical grade GMP conditions. Infusion of CIK cells in patients relapsing after allogeneic stem cell transplanta- 
OS at $1 \mathrm{yr}=70 \%$

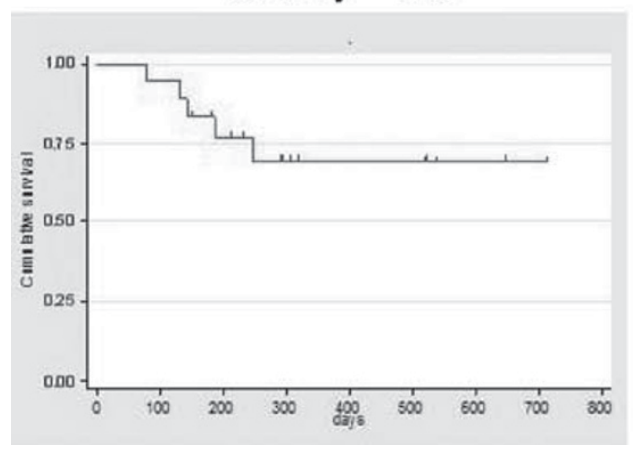

tion (alloHSCT) is well tolerated and significant clinical responses have been reported (Introna, Haematologica 92:952, 2007, Laport, BBMT 2011).

Methods: We performed a new open-label, multicenter phase IIA study (ClinicalTrial.gov NCT01186809) to evaluate the safety (acute and chronic GVHD) and efficacy of a sequential administration of donor derived unmanipulated lymphocytes (DLI) followed by escalated dose of CIK cells in patients relapsing after alloHSCT. Up to 4 combinations of dose escalating levels were provided in sequential order with the intent to define the maximal tolerated dose (MTD).

Results: So far 20 patients have been enrolled and 18 are evaluable. No DLT (grade IV acute GVHD) was observed at any dose. Nine patients have received the highest dose of CIK cells and they all completed the schedule. Only one patient suffered from grade III skin and gut acute GVHD. We observed 6 CR (5 AML, 1MM), $6 \mathrm{PR}$ (3 AML, $2 \mathrm{MM}$ and $1 \mathrm{HD}$ ) and $4 \mathrm{NR}$ (4 AML) and $2 \mathrm{PD}$ (1 NHL, $1 \mathrm{MM}$ ) in the 18 evaluable patients. Overall disease response rate was $66 \%$ and $78 \%$ at the highest dose level. With a median follow up of 270 days (range 80-712), 13 patients $(72 \%)$ are alive and of those $7(54 \%)$ in CR while 6 (46\%) with active disease; 5 (28\%) patients died, 4 for PD and one of fungal lung infection. One year OS and PFS were of $70 \%$ and $48 \%$, respectively.

Conclusions: The sequential infusion of low dose DLI and CIK cells proved feasible, safe and effective with an MTD that has not been reached. We are now looking at further increase the total amount of CIK cells infused to enhance the overall clinical activity of this cellular therapy approach.

\section{3}

Intrahepatic G-CSF stimulated autologous bone marrow stem cell transplantation versus standard of care in patients with decompensated alcoholic liver disease: a randomised study

Y. Chalandon, S. Terraz, V. Kindler, J. Passweg, L. Rubbia-Brandt, A. Hadengue, L. Spahr

University Hospital of Geneva (Geneva, $\mathrm{CH}$ )

Background: Decompensated alcoholic liver disease carries a poor prognosis, due in part to an impaired liver regeneration. We explored in a randomized trial the potential of G-CSF stimulated autologous bone marrow stem cells (aBMT) to improve liver insufficiency over a 3-month period after clinical decompensation. Patients/Methods: 58 patients (M/F: 34/24, mean age 54 yrs) admitted for decompensated alcoholic cirrhosis (mean MELD 19) and alcoholic steatohepatitis were randomized early after

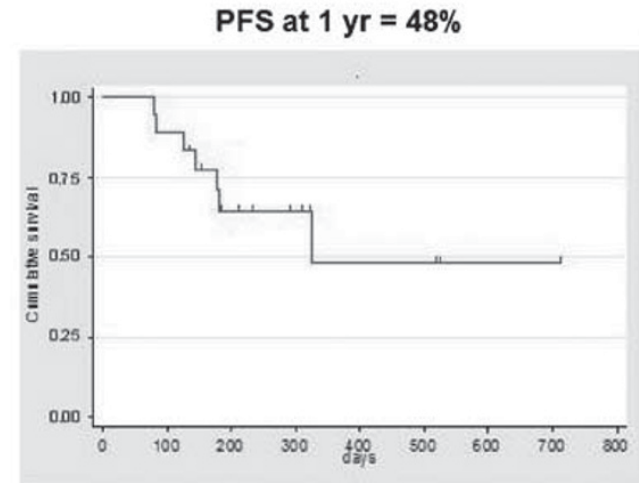

admission to 2 treatment arms. Standard medical therapy (SMT) alone $(n=30)$, was compared to combined SMT with aBMT $(n=28)$. aBMT included a 5-day G-CSF $(10 \mathrm{mcg} / \mathrm{kg}$ sc daily $)$ mobilization prior to BM aspiration ( $\sim 90 \mathrm{ml})$ under propofol sedation, BM MNC isolation (Ficoll $($ ) and reinfusion within the same day in the hepatic artery. A second liver biopsy was performed at 4 weeks to assess histological changes. The MELD score and clinical outcome were determined at 1,2 and 3 months of followup. The primary endpoint was a decrease of $>3$ on the MELD score at 3 months as compared to the initial score.

Results: Patients characteristics were similar at baseline. BM could be administered in all but 2 patients. BM infused contained a mean of $0.47 \times 10 \mathrm{e} 8 \mathrm{MNC} / \mathrm{kg}$. During follow-up, 6 patients died ( 2 in aBMT group, 4 in SMT group), and 9 patients (aBMT: 4; SMT: 5) resumed alcohol consumption. Adverse events were equally distributed in both groups, unrelated to aBMT. The primary endpoint was reached in 19/28 (66\%) aBMT patients, and $17 / 30(57 \%)$ SMT patients (see table). This difference was not statistically significantly different. Regenerations markers at histology and in serum showed similar evolution over time.

Conclusions: Autologous BMT in vulnerable patients hospitalized for decompensated alcoholic liver disease was well tolerated but did not improve liver function nor stimulated liver cell proliferation over a 3-month period. These is in contrast but not in opposition with our previous study in which repetitive G-CSF injections without aBMT infusion were associated with hepatic progenitor proliferation at day 7 . Thus long-term liver regeneration is more difficult to achieve than short-term hepatic progenitor stimulation, which is readily achievable with growth factor administration. The present results suggest either an insufficient stimulus or a resistance of the diseased liver to regenerate.

\section{$\mathrm{O} 284$}

$\mathrm{NiCord} \circledast$ expanded haematopoietic progenitor cells are capable of prolonged myeloid and lymphoid engraftment following myeloablative dual umbilical cord blood transplantation

M. Horwitz (1), J. Chao (1), A. Rizzieri (1), D. Long (1), M. Sullivan (1), C. Gasparetto (1), P. Chute (1), A. Morris (1), C. McDonald (1), D. Snyder (2), E. Galamidi (2), O. Srur-Kidron (2), H. Shoham (2), E. Landau (2), E. Friend (2), J. Kurtzberg (1), T. Peled (2)

(1)Duke University (Durham, US); (2)Gamida Cell Ltd. (Jerusalem, IL)

Human transplantation of expanded HPCs resulting in durable, robust donor myeloid and T-cell engraftment has yet to be

[0283] Table

\begin{tabular}{|l|l|l|l|l|}
\hline & MELD baseline & MELD month 1 & MELD month 2 & MELD month 3 \\
\hline aBMT & $19 \pm 0.8$ & $15.8 \pm 1.2^{\star}$ & $13.7 \pm 0.9^{\star}$ & $13.7 \pm 1.2^{\star}$ \\
\hline SMT & $18.6 \pm 0.7$ & $14.9 \pm 1.1^{\star}$ & $13.1 \pm 1^{\star}$ & $12.5 \pm 1.1^{\star}$ \\
\hline
\end{tabular}


reported. We report here preliminary results of a pilot study of myeloablative dual UCB transplantation where one UCB unit is expanded ex vivo using the NiCord technology. The NiCord UCB graft consists of an expanded AC133+ and a re-frozen unexpanded AC133- fraction. HPCs were expanded for 21 days in media containing hematopoietic cytokines supplemented with nicotinamide (NAM), a vitamin B3 derivative that delays differentiation and improves engraftment efficiency of human HPCs. Patients were conditioned with TBI (1350cGy) and fludarabine $160 \mathrm{mg} / \mathrm{m}^{2}$. GVHD prophylaxis consisted of tacrolimus and mycophenolate mofetil. Five patients (med age 57; range 41-61) with $\operatorname{AML}(n=3)$ or MDS $(n=2)$ received a NiCord-containing dual UCB graft. The unmanipulated unit was HLA 4/6 $(n=4)$ or $5 / 6(n=1)$ matched with the patient and contained a median cryopreserved TNC dose of $3 \times 10^{7} / \mathrm{kg}$ (range 2.3-3.9). The expanded unit was $4 / 6$ matched $(n=3)$ or 5/6 HLA-matched $(n=2)$ with the patient and contained a median cryopreserved TNC dose of $2.2 \times 10^{7} / \mathrm{kg}$ (range 1.7-3.4). After expansion of the AC133+ cell fraction, the TNC and CD34+ cell dose increased a median 486 fold (range 321-577 fold) and 82 fold (range 46-100 fold), respectively. Patients received the NiCord unit that contained a median TNC and CD34+ cell dose of $2.5 \times 10^{7} / \mathrm{kg}$ (range 1.0-4.3) and $3.0 \times 10^{6} / \mathrm{kg}$ (range 1.6-3.9), respectively. The transplanted negative fraction contained a median of $1.3 \times 10^{6} \mathrm{CD} 3+\mathrm{T}$-cells/ $\mathrm{kg}$ (range 0.8-2.8). All patients are durably engrafted, with a median time to ANC $>500$ of 14 days (range 11-26) and platelets $>20,000$ of 33 days (range $30-49$ ). Peripheral blood CD3+ and CD15+ cell chimerism from the 5 patients at the time of last followup are reported. Pt. 1, day +280 ; CD3 (NiCord- $2 \%$, unmanipulated-80\%, host-18\%), CD15 (NiCord-64\%, unmanipulated-36\%). Pt. 2, day +180; CD3 (NiCord-100\%), CD15 (NiCord-100\%). Pt. 3, day +120; CD3 (NiCord-92\%, host-8\%), CD15 (NiCord-90\%, host-10\%). Pt. 4 and 5, day +60; CD3 (unmanipulated-100\%), CD15 (unmanipulated-100\%). There were no infusion-related or unexpected adverse events and all patients are alive and well without GVHD. These data demonstrate prompt and prolonged engraftment of both myeloid and lymphoid cells derived from expanded HPCs (NiCord) in 3 of 5 patients treated to date. Longer follow-up is required to confirm durability.

\section{Working Party Session Infectious Diseases}

\section{5}

\section{Introduction of the Chairman to activities and initiatives} of IDWP

S. Cesaro, C. Cordonnier, J. Styczynski, H. Einsele, D. Engelhard, P. Donnelly, K. Ward, J. Maertens, A. Ullmann, M. Rovira, R. Martino, H. Hirsch, P. Hubacek, M. Aljurf, P. Ribaud, R. De La Camara, G. Tridello, P. Ljungman on behalf of Infectious Disease Working Party

Background: EBMT is a leading society in the field of hematopoietic stem cell transplantation that aims, among other things, to foster clinical research among the affiliated centres.It would help the EBMT to identify potential areas of interest for cooperative research if it was known which topics its own members find important enough to investigate in clinical studies.

Objectives: To assess the type and quality of investigator contribution to EBMT 2012 in the field of infectious diseases.

Methods: The abstracts were categorized as follows: type (prospective, retrospective, case report, meta-analysis), origin (single centre, multicentre), population (adult, paediatric, adult and paediatric), type of transplant (autologous, allogeneic, autologous and allogeneic), infection (bacteria, fungi, virus, other), and main objective (epidemiology, diagnosis, prophylaxis, therapy, adoptive immunotherapy, immunity/immune recovery, risk factor/survival, other).
Results: 77 (8\%) of the 930 abstracts submitted by physicians $(8 \%)$ were had infectious complications as their topic. $45(58 \%)$ were retrospective studies, $23(30 \%)$ were prospective studies, 7 were case reports, and there was 1 in-vitro study and 1 metaanalysis. Only 2 abstracts reported the results of multicentre studies, 1 from EBMT-IDWP and 1 from GITMO. 36 abstracts $(47 \%)$ concerned adults, $17(22 \%)$ were focused on paediatrics, 8 on mixed populations and the population was not defined in 16 abstracts (21\%). 52 abstracts $(67.5 \%)$ focused on allogeneic transplant, $5(6.5 \%)$ on autologous transplant, $11(14 \%)$ on allogeneic/autologous transplant, and the or was not applicable in 1 abstract. Viral infections were studied in $37(48 \%)$ abstracts, fungal infections in $18(23 \%)$, bacterial infections in $8(10 \%)$, and various infections in $10(13 \%)$ with pneumonia and vaccination being the focus of 2 and 1 abstracts, respectively. ( 1 was not applicable) The objective of $11(14 \%)$ abstracts was epidemiology, $11(14 \%)$ risk factors/survival, diagnosis $13(17 \%)$, prophylaxis $16(21 \%)$, immunity/immune recovery $9(12 \%)$, therapy $9(12 \%)$, adoptive immunotherapy $2(3 \%$,$) the remaining$ 6 having miscellaneous objectives.

Conclusion: Most abstracts reported retrospective studies involving single-centres, with viral and fungal infections representing the most frequent areas of interest. This information should prove useful in helping plan EBMT-wide prospective, multicentre studies.

\section{6}

ECIL 4 initiative: highlights of 2011 meeting

C. Cordonnier, P. Ljungman, O. Marchetti, J. Maertens, M. Akova, A. Groll, J. Donnelly, H. Einsele, R. Herbrecht, G. Petrikkos, A. Cometta, D. Engelhard, F. Menichetti, J. Bille, S. Bretagne, C. Viscoli, S. Cesaro, T. Calandra on behalf of the IDWP

The first European Conference on Infections in Leukemia (ECIL1) was organized in France in 2005 under the auspices of the Infectious Diseases Working Party of the European Group for Blood and Marrow Transplantation (EBMT), the Infectious Diseases Group of the European Organisation for Research and Treatment of Cancer (EORTC), the Supportive Care Group of the European LeukemiaNet (ELN) and the International Immunocompromised Host Society (ICHS). The Conference objective was to elaborate guidelines for the prevention, diagnosis and management of infectious complications arising during the treatment of leukaemia and following hematopoietic stem cell transplantation (HSCT). Since then, ECIL meetings have been held every 2 years, the last one (ECIL4) being held in September 2011. On each occasion, 50 to 60 experts from the fields of haematology, oncology, infectious diseases, microbiology and clinical trials from 28 countries - mostly European, and also Israel and Australia - were invited to participate. Each presentation and set for guidelines were prepared by a designated working group, who adopted the quality of evidence and strength of recommendation grading system of the Centers for Diseases Control and presented their proposals during a plenary session at the ECIL meeting. These were then modified according to the discussions that followed until a consensus was reached. This was then made available to the medical community as a slide set on each of the web sites of the organizations involved: www. ebmt.org. www.eortc.be, www.ELN.org and www.ichs.org.

Since ECIL1, 4 bacterial topics, 6 fungal topics, and 3 viral topics have been addressed, and 14 papers have been published or are in press. Additionally, as guidelines by definition have rather short life-expectancies, most guidelines, especially the fungal ones, have been updated on a 2-year basis.

One of the objectives of ECIL is also to identify new areas of research. For example, at the ECIL4 meeting held in September 2011, 3 new topics were addressed: (1) Management of respiratory virus and adenovirus infections (2) Bacterial resistance in haematology. (3) Specific consideration on fungal disease and antifungal treatment in children. The core of the guidelines on these 3 topics will be presented at the IDWP session. We 
hope that the ECIL guidelines will help HSC transplanters to make rationale, evidence-based choices when managing the infections their patients encounter.

\section{1}

Use of rituximab for post-SCT EBV-PTLD: the final results of a retrospective EBMT study

J. Styczynski, L. Gil, J.P. Donnelly, P. Ljungman, R. Martino, S. Halkes, K. Theunissen, J. Maertens, K. Kalwak, P. Hubacek, S. Sica, W. van der Velden, H. Omar, C. Nozzoli, F. Fagioli, S. Matthes, M.A. Diaz, M. Migliavacca, A. Balduzzi, M. Faraci, A. Tomaszewska, R. de la Camara, A. van Biezen, G. Tridello, $H$. Einsele, S. Cesaro for Infectious Diseases Working Party of European Blood and Marrow Transplantation Group

Background: EBV-related Post-Transplant Lymphoproliferative Disorder (EBV-PTLD) is a life threatening complication after allo-HSCT. The objective of this study was to investigate prognostic factors that influence the outcome of PTLD after a rituximab-based treatment.

Patients and Methods: Data of 4529 allo-HSCT from 19 centres were included in the study. PTLD was diagnosed in 144 cases (55 children, 89 adults), as biopsy-proven in 85 (59\%) cases or probable disease in $59(41 \%)$ cases. The median age at transplant was 22 years (range, 0.3-67); 22 cases were HLA-identical siblings (MFD), 13 mismatched family/haplo (MMFD/haplo), 70 match unrelated donor (MUD) and 39 mismatched unrelated donor (MMUD); the source of stem cells was: 108 peripheral blood (PB), 22 bone marrow (BM) and 14 cord blood (CB). Patients were treated weekly with a median of 3 doses of rituximab (range, 1-16).

Results: The overall EBV-PTLD incidence was $3.2 \%$, and it ranged from $1.2 \%$ in MFD-SCT, $2.9 \%$ in MMFD/haplo-SCT, $4 \%$ in MUD-SCT, $4.1 \%$ after CB-HSCT, and to $11.2 \%$ in MMUDSCT. EBV-PTLD occurred at median of 2 months (range, 0.281 months) after HSCT. PTLD resolution after rituximab was observed in $69 \%$ of patients. By multivariate analysis, risk factors influencing the response of PTLD to rituximab were age $>30$ years and involvement of extra-lymphoid tissue. In the prognostic model, an increasing number of risk factors worsened the outcome: 100-days PTLD survival with 0,1 or 2 factors: $93 \%, 66 \%$ and $39 \%$, respectively $(\mathrm{P}<0.001)$. Immunosuppression tapering was associated with a higher proportion of survival after PTLD (86\% v 60\%). Chemotherapy did not improve survival in no-RI arm. An increase of serum EBV-DNA-emia during therapy was predictive of poor survival after PTLD, while a decrease after one week predicted good outcome.

Conclusions: Over two-thirds of patients with EBV-PTLD responded to treatment with rituximab. Age over 30 years, extra-nodal disease, and absence of early response to rituximab are associated with a poor prognosis.

\section{2}

Intratecal rituximab for CNS-EBV PTLD: a new retrospective study

J. Styczynski (1), K. Czyzewski (1), A. Krenska (1), R. Debski (1), O. Zajac-Spychala (2), J. Wachowiak (2), M. Wysocki (1) (1)Nicolaus Copernicus University (Bydgoszcz, PL); (2)Medical University (Poznan, PL)

Background:Post-transplantlymphoproliferativedisorder(PTLD) with central nervous system (CNS) involvement is a rare complication after hematopoietic stem cell transplantation (HSCT). It is caused by uncontrolled B-lymphocyte proliferation following latent reactivation of Epstein-Barr virus (EBV). Rituximab administration is the therapy of choice in systemic PTLD. So far there are scanty data on intrathecal (ITH) rituximab administration in PTLD patients with CNS involvement.

Objective: Analysis of safety and efficacy of intrathecal therapy with rituximab in children with CNS involvement of PTLD.
Patients and Methods: 8 patients (ALL-2, SAA-2, AML-2, ABL-1, ES-1), aged 5-19 years, were included to the study. PTLD occurred 22-153 days after allo-HSCT (4 MUD, 3 MMUD, 1 MFD). Diagnosis of PTLD was based on clinical symptoms and quantitative EBV-DNA load in serum and in cerebro-spinal fluid (CSF), obtained by PCR. Therapy of PTLD: weekly rituximab $375 \mathrm{mg} / \mathrm{m}^{2}$ iv with simultaneous ITH administration until resolution of clinical symptoms and EBV-DNA-emia negativity. Rituximab in dose $10-30 \mathrm{mg}$, solved in $3-10 \mathrm{ml}$ of $0.9 \% \mathrm{NaCl}$ was administrated ITH for 2-10 minutes, 1-12 times in 7 days intervals.

Results: All patients had systemic PTLD with CNS involvement. Clinical symptoms of CNS involvement were presented in 7 of 8 patients as symptoms of encephalitis: nausea, fever, loss of memory, consciousness disturbances, fatigue, loss of appetite. In 1 patient, unusual fatigue was the only symptom of CNS involvement. Other symptoms of systemic PTLD included: lymphoid tissue involvement $(n=6)$, hepatomegaly $(n=4)$, and fever $(n=5)$. MRI was performed in 3 patients and revealed: (i) $5 \times 6 \mathrm{~mm}$ focal change in occipital cortex of brain in one patient, (ii) $1.5 \mathrm{~cm}$ tumor-like change located between pons and cerebellar peduncles, (iii) cortical atrophy. Serum EBV-DNA-emia at diagnosis: $3.44 \times 10 \mathrm{E} 5-7.5 \times 10 \mathrm{E} 9 \mathrm{gc} / \mathrm{mL}$. CSF EBV-DNA load at the diagnosis: $4.10 \times 10 \mathrm{E} 3-3.77 \times 10 \mathrm{E} 7 \mathrm{gc} / \mathrm{mL}$. CSF leukocytosis 2-36/mcL, protein 34.4-191 mg/dL. In 6 patients serum EBVDNA-emia was negative after 2-5 rituximab doses. CSF EBVDNA negativization was observed after: 1 rituximab dose in 3 patients, 2 rituximab doses in 2 patients, 3 rituximab doses in one patient and in one patient after 8 rituximab doses. Five patients achieved complete remission, 2 patients achieved partial remission (1 patient without symptoms, slight progression changes in MRI, CSF EBV-DNA positive; 1 without clinical symptoms, tumor-like focus observed in MRI, CSF EBV-DNA negative). One patient has died after clinical progression of PTLD, although EBV-DNA serum load decreased by 2 logs after one-week therapy. Seven patients had good tolerance of rituximab ITH without post-dural puncture symptoms. Adverse events were observed in one patient: seizures during third rituximab ITH infusion.

Conclusions: Rituximab intrathecal administration is a safe and effective method of therapy of CNS involvement of PTLD. Further studies of this treatment modality are necessary.

\section{Working Party Session Inborn Errors}

\section{8}

Management of DOCK8 deficiency by haematopoietic stem cell transplantation

M.H. Albert (1), S. Aydin (1), S. Matthes-Martin (2), M. Hönig (3), A. Schulz (3), C. Picard (4), V. Barlogis (5), A. Gennery (6), M. Ifversen (7), J. van Montfrans (8), T. Kuijpers (9), R. Bredius (10), G. Dückers (11), W. Al-Herz (12), A. Metin (13), S. Pai (14), R. Geha (14), TC. Bittner (1), G. Notheis (1), J. SawalleBelohradsky (1), V. Heinz (1), B. Gathmann (15), K. Engelhardt (16), B. Grimbacher (15), ED. Renner (1), T. Chatila (17), BH. Belohradsky (1), HD Ochs (18), B. Gaspar (19)

(1)Dr. von Haunersches Kinderspital der LMU (Munich, DE); (2)St. Anna Kinderspital (Vienna, AT); (3)Universitätskinderklinik (UIm, DE); (4)Hopital Necker (Paris, FR); (5)Hopital Timone Enfants (Marseille, FR); (6)Institute of Cellular Medicine (Newcastle upon Tyne, UK); (7)Rigshospitalet (Copenhagen, DK); (8)UMC (Utrecht, NL); (9)AMC (Amsterdam, NL); (10)UMC (Leiden, NL); (11)Helios Klinikum (Krefeld, DE); (12)Kuwait University (Kuwait, KW); (13)Ankara University (Ankara, TR); (14)Childrens Hospital (Boston, US); (15)CCI (Freiburg, DE); (16)Royal Free Hospital (London, UK); (17)UCLA (Los Angeles, US); (18)Childrens (Seattle, US); (19)UCL Institute of Child Health (London, UK)

In 2009, mutations in the gene for dedicator of cytokinesis 8 (DOCK8) have been identified as the cause of the autosomal 
recessive variant of Hyper-IgE syndrome. The clinical presentation of this primary combined immunodeficiency is characterized by eczema, debilitating viral infections of the skin, chronic mucocutaneous candidiasis, pulmonary infections, severe allergies, vascular complications and a high risk for malignancy. While the long-term prognosis of affected patients is not yet clearly defined, the high morbidity and mortality of this disease suggest HSCT as a potential curative measure. Based on data from the ongoing DOCK8 therapy survey, we retrospectively studied the outcome of HSCT in patients with DOCK8 mutations.

A total of 22 patients from 11 institutions were identified, 4 of whom had been previously reported. Three patients had malignant disease at the time of transplantation (2 cutaneous T-cell lymphomas, 1 EBV-LPD). At a median age of 11 years (3-18) transplantation from a MUD $(n=10)$, MFD $(n=1)$, MMFD $(n=2)$ or MSD $(n=9)$ was carried out. After a median follow-up of 6 months (2-91) the overall survival is $73 \%(16 / 22)$. Three patients had grade III-IV acute GVHD. Causes of death were relapsing malignancy $(n=2)$, GVHD $(n=2)$, graft failure $(n=1)$ and sepsis $(n=1)$. T-cell chimerism at last follow-up was $>99 \%$ in $12 / 14$ evaluable patients and $50-90 \%$ in $2 / 14$. Of the 16 surviving patients all 9 evaluable had complete correction of their immunodeficiency. Other symptoms such as eczema, allergies, mollusca, bacterial infections, fungal infections and pulmonary function deficits disappeared or vastly improved in all evaluable patients. Two post transplant malignancies were observed: one thyroid carcinoma 7 years post transplant with a TBI-containing conditioning regimen and one squamous cell carcinoma diagnosed shortly after transplant in previously inflamed skin lesions. Both patients are alive.

Longer follow-up will be needed to ascertain that HSCT will correct the malignancy risk, as the DOCK8 molecule has been implicated as a tumor suppressor and is expressed in extrahematopoietic tissues. A survey to define the natural course of disease and to guide treatment recommendations for all patients is currently underway on behalf of ESID and EBMT. In summary, HSCT corrects the immunodeficiency and other disease manifestations in DOCK8 deficiency and this treatment should be offered at least to all patients with severe disease manifestations.

\section{Working Party Session Lymphoma}

303

Jian-Jian Luan Award

CMV-replication after allogeneic stem cell transplantation

is associated with a GvHD-independent reduced relapse risk in lymphoma: evidence for a putative virus-versus-lymphoma effect

A. Elmaagacli, M. Koldehoff, D. Janson, N. Steckel, M. Lindemann, M. Ditschkowski, T. Gromke, S. Christoph, R. Trenschel, R. Ross, D. Beelen

University Hospital of Essen (Essen, DE)

We have previously showed that a CMV- reactivation after HSCT is associated with a reduced risk for leukemic relapse in pts with AML. Further, Erlach et al. showed in a lymphoma mice transplant model that coinfection with $\mathrm{mCMV}$ induced a strong anti-lymphoma effect by induction of apoptosis in lymphoma cells, which consecutively improved OS in mice.

This prompted us to investigate the influence of replicative CMV infection in 94 (median age 45, 18-70) pts with lymphoma, who received transplants from unrelated $(n=67,71 \%)$ or related $(n=27,29 \%)$ donors. Pts were transplanted from HLA-ident. $(n=74)$, HLA-MM $(n=16)$ or HLA-haploident. SIB $(n=4) .13$ pts $(14 \%)$ were transplanted for indolent lymphoma ( $F L n=11, C L L$ $n=2), 67$ pts $(79 \%)$ for aggressive lymphoma (B-lineage $n=35$, T-lineage $n=27$, transformed $n=5), 11$ pts $(12 \%)$ for $M C L$ and
3 pts (3\%) for HD. The disease status of pts at HSCT was CR in 20 pts, PR in 40 pts, refractory in 30 pts and untested in 2 pts. 55 pts (59\%) received previous autograft and 82 pts $(87 \%)$ were treated prior to transplant with at least 3 chemotherapy lines. The HCT-Cl were $0-2$ in 76 pts $(81 \%)$ and $3+$ in 18 pts (19\%). Myeloablative conditioning was applied in 60 pts $(64 \%)$ while 34 pts $(36 \%)$ received RIC. $68 \%$ of pts $(n=48)$ were at risk for CMV reactivation. CMV replication as detected by pp65 antigenemia assay occurred in 34 pts (36\%).

Taking all competitive risks into account, the cumulative incidence of PFS at 5 yrs after HSCT was $62 \%$ (95\% CL: $31-45)$ in pts without as compared to $80 \%(95 \% \mathrm{CL}: 9-31)$ in pts with pp65 antigenemia $(p<0.018)$. In multivariate analysis including all effecting factors, CMV replicative status was confirmed as a strong independent predictor of PFS (HR: $0.29,95 \% \mathrm{CL}$ : $0.08-1.00, p<0.049$ ) together with chronic GvHD (HR: $0.32,95 \%$ CL: $0.13-0.80, p<0.016$ ), and chemorefractory (HR: $3.3,95 \% \mathrm{CL}$ : $1.28-8.4, p<0.013)$. The anti-lymphoma effect was detectable across all lymphoma subsets and was most pronounced in pts with chemotherapy refractory lymphoma. However, OS rate and aGVHD grade $2-4$ did not differ in both groups $(52 \%$ for pts with CMV-R. vs $51 \%$ without, n.s., and $39 \%$ vs $35 \%$, n.s, respectively), whereas 5-yr NRM was higher in group with CMV-repl. (42\% vs $23 \%, p=0.05)$.

This is the first report which demonstrates a strong and GVHDindependent effect of CMV replication on the PFS in pts with lymphoma, which deserves further prospective studies.

\section{Chronic Graft-versus-host Disease}

\section{5}

Efficacy of standardised and quality controlled cord blood serum eye drop therapy in the healing of severe corneal epithelial defects in GvHD-related dry eye G. Tolomelli (1), P. Versura (1), M. Buzzi (1), P. Stancari (1), F. Bonifazi (1), G. Bandini (1), M. Arpinati (1), E. Campos (1) (1)"L\&A.Seragnoli", Policlinico Sant'Orsola-Malpighi (Bologna, IT); (1)Policlinico Sant'Orsola-Malpighi (Bologna, IT)

Purpose: To evaluate the efficacy of a standardized and qualitycontrolled Cord Blood Serum (CBS)-based eye drop preparation in the short term treatment of corneal epithelial damage in patients with severe dry eye (DE) and chronic Graft Versus Host Disease (GVHD) after allogeneic hematopoietic stem cell transplantation.

Methods: Patients with GVHD-related DE not responding to previous autologous serum therapy, were enrolled in the frame of a registered clinical trial (Clin Trial Gov Id NCT01234623). Sterile CBS eye drops were prepared to contain $1.6 \mathrm{ng} / \mathrm{ml}$ EGF in a one-day-dose dispensing device, and were administered for one month. Extent of corneal epithelial defect was evaluated in $\mathrm{mm} 2$ /area, subjective symptoms (OSDI score), Schirmer test I (ST), Break Up Time (BUT), tear osmolarity, corneal esthesiometry (Cochet-Bonnet esthesiometer), conjunctival scraping and imprint cytology with goblet cell (GC) count were performed at baseline (V0), after fifteen (V1) and thirty (V2, endpoint) days of treatment. Satisfaction and tolerability questionnaires were evaluated at V1 and V2.

Results: between January 2010 and April 2011 we treated 17 patients with severe dry eye (all DEWS score 4) after allogeneic HSCT. Seven patients had extensive chronic GVHD. Only 5 $(29 \%)$ were on systemic immunosuppressive treatment. All patients had been previously treated with autologous serum, whereas half the patients had received topical cyclosporine A. A significant reduction was shown at the endpoint $v s$. baseline in corneal epithelial damage (media+SD: $13.6 \pm 14.2$ vs. $36.7 \pm 29$ $\mathrm{mm}^{2} /$ area, respectively), discomfort symptoms (OSDI score $24.2 \pm 8.8$ vs. $42.3 \pm 15.4)$, scraping cytology score $(3.9 \pm 1.4$ vs. $6.6 \pm 2.5)$, tear osmolarity (313.7 \pm 6 vs. $324 \pm 7.7 \mathrm{mOsm} / \mathrm{L})$ 
(p always $<0.0001$ ) while a significant improvement was shown in corneal esthesiometry $(48.2 \pm 1.8$ vs. $49.7 \pm 1.1 \mathrm{nylon} / \mathrm{mm} / \mathrm{length}$, $p<0.05)$. The extent of initial corneal damage but not duration of epithelial defects correlated with response $(p<0.0001)$. No patient withdrew or presented adverse event. All patients reported a high satisfaction degree upon eye-drops instillation.

Conclusions: Our data demonstrate that CBS eye drops may represent a new therapeutic approach for the healing of severely injured corneal epithelium in patients with chronic GVHD. A longer follow up is needed to confirm the duration of the efficacy after CBS treatment.

\section{6}

Preliminary results of a phase II trial of montelukast for the treatment of Bronchiolitis obliterans syndrome after HSCT and implications for immunobiology of disease

K. Williams (1), S. Pavletic (1), S. Lee (2), F. Hakim (1), S. Mitchell (1), B.L. Manning-Geist (1), J. Gea-Banacloche (1), L. Comis (1), E. Cowen (1), K. Baird (1), J. Shelhamer (3), B. Blacklock-Schuver (1), D. Avila (1), D. Zulchinski (1), R. Gress (1)

(1)National Cancer Institute (Bethesda, US); (2)Fred Hutchinson Cancer Research Center (Seattle, US); (3)National Institutes of Health (Bethesda, US)

Bronchiolitis obliterans syndrome (BOS) after allogeneic HSCT is a serious manifestation of cGVHD with high mortality. We present preliminary results from an IRB-approved prospective, open label, phase II trial to test the efficacy of montelukast, a leukotriene inhibitor, for the treatment of BOS after HSCT and to elucidate the biology of BOS. BOS diagnostic criteria included: FEV $1<75 \%, F E V 1 / V C<0.7$ or air trapping on CT and $\mathrm{RV}>120 \%$ or RV/TLC $>120 \%$ in the absence of infection, presence of another cGVHD manifestation and lack of response to prior therapies. Twenty-one patients have enrolled. One withdrew prior to medication and $17 / 21$ patients have reached the primary endpoint (6 months) on study medication (10 mg qhs). Study participants ranged from 15-64 years, $12 / 20$ female, with baseline FEV1 of $24-73 \%$ predicted, and median FEV1/VC $0.5(0.29-0.78)$. All patients met criteria for treatment success $(<15 \%$ decline in 6 months), with FEV1 changes of: increased $5-13 \%$ predicted $(n=5)$, stable with change $<5 \%(n=7)$, and declined $5-13 \%(n=5)$. Comparison of patient pre-study FEV1 decline to on-study FEV1 values was generated using the slope of FEV1 volume vs. days post-transplant. The difference in pre- and primary endpoint slope revealed: 15/17 improved. Six minute walk test demonstrated 4 significant increases, 11 stable, 2 significant decline. Lung function score was decreased in 1 , increased in 4 , and stable in remainder. Other manifestations of cGVHD showed: 4/8 GI GVHD improved and 4/8 stable; $3 / 6$ liver cGVHD improved, 3/6 stable, 1 worse, using $\mathrm{NIH}$ consensus staging. Of 10 patients enrolled $>2$ years ago, $70 \%$ are alive, with 2 durable improvements $(6,14 \%)$ and 2 stable from baseline, compared with historical controls of $44 \%$ survival at 2 years. At the primary endpoint, available preliminary data show increases in proportion of CD8 T cells with Cysteinyl receptor expression in patients with improvements in FEV1 suggesting a possible link between receptor expression and disease modification by montelukast. At 1 year, CD4/CD8 ratios improved in 5 and normalized in 3 patients, suggesting improving immune function with prolonged montelukast therapy. Montelukast was well-tolerated with only one grade II probable attributable adverse event during the collection period. These findings suggest that montelukast is a promising therapy for BOS after allogeneic HSCT and that the cysteinyl leukotrienes may be a point of regulation for progressive BOS after HSCT.
0317

$\mathrm{NIH}$ lung score components are associated with clinical outcomes, Bronchiolitis obliterans syndrome and survival in chronic GvHD

L. Grkovic (1), D. Pulanic (1), S.M. Steinberg (2), K.M. Williams (2), K. Baird (2), S.A. Mitchell (2), E.W. Cowen (2), M.B. Datiles III (2), D. Aria (2), C. Bassim (2), G. Joe (2), L. Comis (2), J. Baruffaldi (2), D. Zhang (2), C. Sportes (2), D.H. Fowler (2), F. Hakim (2), R.E. Gress (2), S.Z. Pavletic (2)

(1)Clinical Hospital Center Zagreb (Zagreb, HR); (2)National Institutes of Health (Bethesda, US)

Objectives: NIH chronic GVHD (cGVHD) consensus project proposed NIH lung score (NIHLs) 0-3 as a measure of cGVHD severity (BBMT 2005; 11:945). NIHLs is composed of 3 components: respiratory symptoms (RS), FEV1 and lung function score (LFS) devised from DLCO and FEV1. The final NIHLs $(0-3)$ is determined by maximum of individual components. The goal of this study was to evaluate associations of NIHLs components and clinical outcomes.

Methods: 211 cGVHD patients (pts), median age 48y (18-70), enrolled 2004-2011 into the $\mathrm{NCl}$ natural history study, with median follow-up for survivors of 36 months. Pts received 3 (0-9) prior systemic therapies (PST). $66 \%$ had severe and $32 \%$ moderate NIH global cGVHD severity; median 5 (1-8) organs were involved. $42 \%$ had mild, $26 \%$ moderate and $10 \%$ severe NIHLs. 46 pts had bronchiolitis obliterans syndrome (BOS) by modified NIH criteria (BBMT 2010; 16:S106). Measures were obtained at study entry: NIHLs, SF36 (PCS, MCS), Lee symptom scale (total and breathing), HAP (MAS, AAS), walk velocity, grip strength, CGVHD activity, intensity of immunosuppression, number of PST, cGVHD severity by clinician and patient $(0-10)$.

Results: FEV1 and LFS were weakly to moderately correlated with 8-9 outcome measures $(|r|>0.30)$; DLCO with grip strength, AAS and walk velocity $(|r|>0.30)$, and increasing RS and NIHLs was associated with 11 and 12 outcomes respectively $(p<0.05$; most $p<0.01$ ). Since BOS is a manifestation of lung cGVHD we analyzed components of NIHLs relative to BOS. $91 \%$ of pts without BOS can be predicted on the basis of RS alone (symptom score $<2$ ) while only $56 \%$ with BOS could be correctly identified using only RS. $89 \%$ pts without BOS as well as $89 \%$ with BOS could be predicted on the basis of FEV $1<59 \%$ alone. $63 \%$ with and $64 \%$ without BOS can be correctly classified by DLCO alone; $80 \%$ without BOS could be correctly predicted on the basis of the NIHLs alone as could $93 \%$ with BOS (NIHLs $\geq 2$ ). Overall 3yr survival (OS) was $76.2 \%$. In univariate analysis worse RS $(p<0.0001)$, lower FEV1 $(p=0.007)$, DLCO $(p=0.002)$, higher LFS $(p=0.002)$ and NIHLs $(p<0.0001)$ were associated with worse OS. In multivariable analyses only NIHLs retained an association with OS. OS at 3y: $81 \%(95 \% \mathrm{Cl} 73-86 \%)$ vs. $31 \%(95 \% \mathrm{Cl} 14-55 \%)$ for NIHLs $0-2$ vs. 3 respectively.

Conclusion: Most components of the NIHLs are associated with important clinical outcomes. Severe NIHLs is predictive of clinical BOS and poor survival. These data support the validity of NIHLs in pts with cGVHD.

\section{8}

Prospective study on immunosuppressive/ immunomodulatory treatment of patients with newly diagnosed bronchiolitis obliterans syndrome after allogeneic haematopoietic cell transplantation

Z. Kuzmina, V. Petkov, K. Krenn, R. Knobler, N. Worel, P. Kalhs, M. Mitterbauer, W. Rabitsch, A. Schulenburg, G. Dekan, H. Greinix

Medical University of Vienna (Vienna, AT)

Background: Bronchiolitis obliterans syndrome (BOS) following allogeneic $\mathrm{HCT}$ is a manifestation of chronic graft-versushost disease with poor prognosis and 5-year survival around $20 \%$. Treatment and survival of patients with BOS has not 
improved over the last 20 years. Evidence regarding the efficacy of various immunosuppressive therapies in BOS is still sparse. Aim: The purpose of the study was to prospectively diagnose BOS early in the course of the disease by serial assessments of consecutive patients from day 100 after HCT and to compare the efficacy of adjunct ECP with other immunosuppressive treatments in BOS patients newly diagnosed and evaluated uniformly according to the National Institutes of Health consensus criteria.

Patients and Methods: Forty-six patients (24 male, 22 female) with a median age of 41 (range, 19-65) years developed BOS a median of 336 (range, 97-1254) days after HCT. At onset of therapy BOS was mild in $37(80 \%)$ and moderate in $9(20 \%)$ patients. The median FEV1 and the median lung function score (LFS) at onset were $65 \%$ (range, $45-77 \%$ ) and 6 (range, 2-9). Signs of air trapping in HR-CT scans were present in $70 \%$ of patients and BO was proven histologically in 21 (46\%). Therapy of BOS consisted of steroids with or without calcineurin inhibitors (CNI) or sirolimus in all patients whereas $27(59 \%)$ also received adjunct ECP on 2 days every other week. Overall survival (OS) was analysed after a median follow-up of 35 (range, 12-66) months after HCT and 22 (range, 3-54) after diagnosis of BOS.

Results: When assessed after 1 year, 17 of 27 patients $(63 \%)$ responded to adjunct ECP and 12 of $19(63 \%)$ to other immunosuppressive therapies. Complete (CR) and partial resolutions (PR) were achieved in $11 \%$ and $52 \%$ of the ECP cohort compared to $16 \%$ and $47 \%$ of the non-ECP group and not significantly different. Eight patients (80\%) given adjunct ECP as first-line therapy achieved a response $(C R n=3, P R n=5)$ compared to $12(63 \%)$ given first-line therapy with $\mathrm{CNI}$ and steroids alone (CR $n=3, P R n=9)$. OS at 3 years was $86 \%$ for responders and $71 \%$ for progressive and stable disease. Patients with progressive BOS had significantly worse OS at 3 years compared to CR $(44 \%$ vs. $100 \%, p=0.02)$ and $P R$ ( $44 \%$ vs. $83 \%, p=0.006)$.

Conclusion: Early diagnosis and immunosuppressive/ immunomodulatory therapy are able to improve outcome of patients with BOS. Longer follow-up and larger patient numbers are warranted for confirmation of these promising findings.

\section{9}

Imatinib in 34 patients with steroid-refractory chronic graft-versus-host-disease: final evaluation of the second prospective trial, according to the NIH Consensus criteria for cGvHD

A. O. Olivieri (1), M. Cimminiello (2), P. Corradini (3), P. lacopino

(4), F. Patriarca (5), N. Mordini (6), A. Donelli (7), F. Onida (8),

C. Selleri (9), V. Pavone (10), R. Nuccorini (2), S.P. Pascale (2),

S. Coluzzi (2), S. Svegliati (1), A. Gabrielli (1), P. Leoni (1),

I. Olivieri (1), A. Bacigalupo (11)

(1)Università Politecnica delle Marche (Ancona, IT); (2)A.O.R. San Carlo (Potenza, IT); (3)Fondazione IRCCS Istituto Nazionale Tumori (Milan, IT); (4)Azienda Ospedaliera (B-M-M) (Reggio Calabria, IT); (5)A.O.U. Santa Maria della Misericordia (Udine, IT); (6)ASO S. Croce e Carle (Cuneo, IT); (7)Azienda Ospedaliera Universitaria di Modena (Modena, IT); (8)IRCCS di natura pubblica (Milan, IT); (9)Università Federico II (Naples, IT); (10)Pia Fondazione di culto e di religione Card. G. Panico (Tricase (LE), IT); (11)Centro Trapianto di Midollo osseo San Martino Hospital (Genoa, IT)

After our first study in 19 patients, receiving Imatinib as compassionate use for steroid refractory Chronic Graft Versus Host Disease (cGVHD) we conducted a second study in a larger series of patients with steroid-refractory CGVHD, by using the more stringent response criteria suggested by NIH Consensus Conference for cGVHD. Overall 34 patients with cGVHD, refractory to 2 lines of therapy have been enrolled: 23 male, 11 female, median age 49 years (28-73). Median duration of cGVHD was 29 (2-148) months; all patients were heavily pretreated and 19 have been previously treated with ECP and/or Rituximab. Main cGVH targets were lung (27) and skin (generalized scleroderma in 15 and localized in 8); 6 patients had Sicca Syndrome and 12 other visceral involvement. Imatinib was administered at low dose (100-200 mg/day) for a median duration of 16 months (3-45); 15 patients are still on Imatinib.The drug was well tolerated and we did not observe toxic deaths; haematological toxicity was mild, ( 1 patient had grade 3 anemia), while the main grade 3-4 extra-hematological toxicities were: muscle aches (7), dyspnea (4) and skin rash (3). Response rate (RR), evaluated according to NIH criteria, was $45 \%$ at 6 months and $48 \%$ at 12 months; skin Body Surface Area (BSA) involvement was significantly reduced in $43 \%$ of cases, with a reduction of the Rodnan score in $67 \%$ of cases; lung functional score (LFS) improved in $47 \%$ of patients and Gastrointestinal score in $63 \%$; among 15 patients receiving steroids, 11 were able to

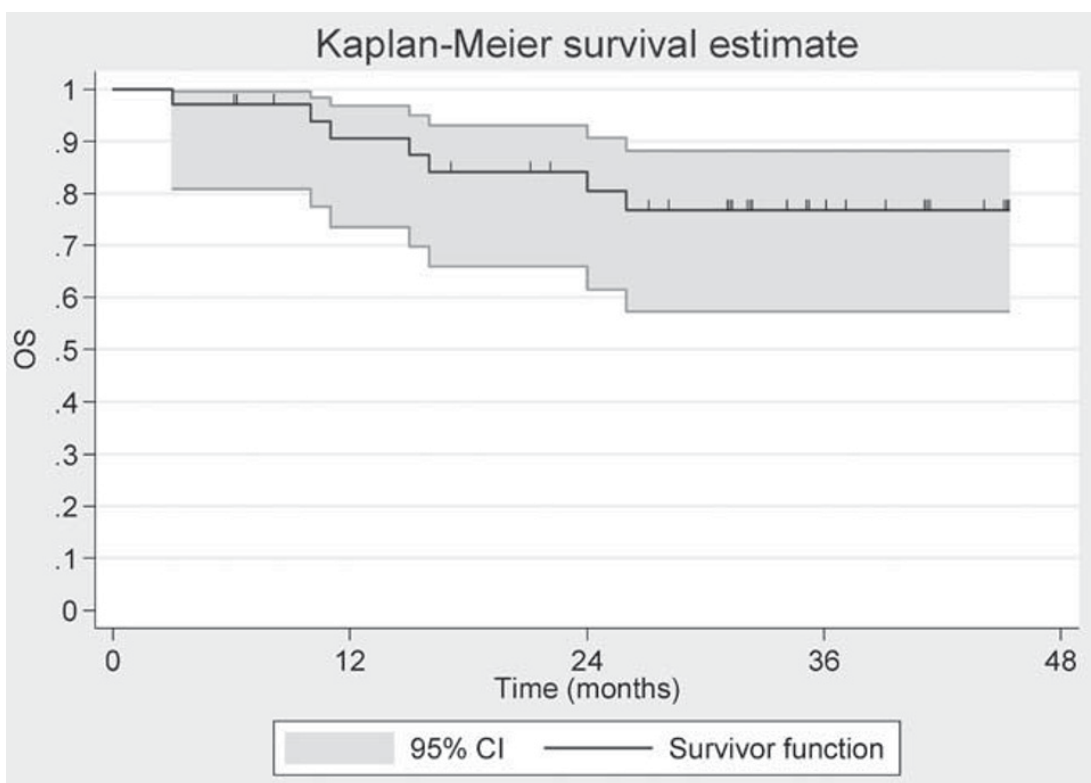




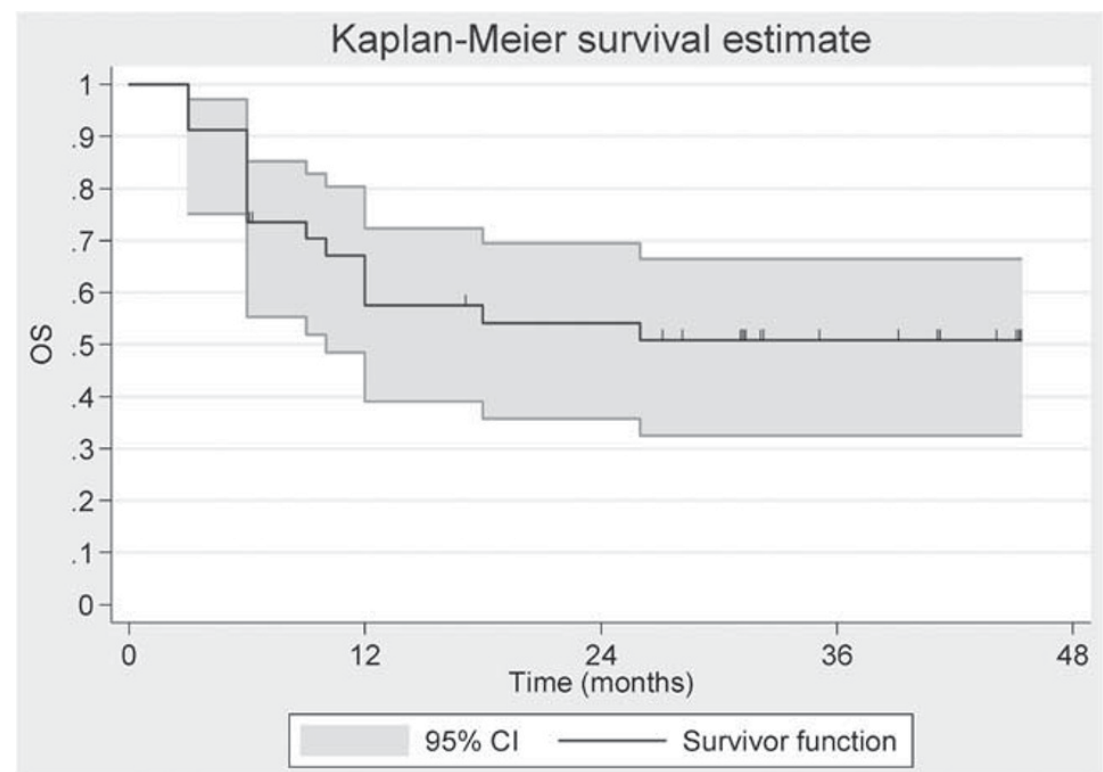

stop or significantly reduce steroids. With a median follow-up of 31 months (3-45) 27 patients are alive and 7 patients died: 4 due to GVHD progression and 2 for infections; 17 patients are in continue response at last follow-up, without additional treatments. The RR observed in this second trial, in a larger series of patients with steroid-refractory cGVHD seems slightly inferior, compared to the RR observed in the first trial, probably due to the more stringent response criteria used. Moreover the higher median age and the different kind of patients could have influenced these results: the previous study included only patients with skin fibrotic cGVHD and pediatric patients. However the stable response observed after 12 months and the promising outcome (Figure 1: OS; Figure 2: EFS), in this very hard to treat set of patients, suggest that Imatinib is a valuable option in patients with steroid-refractory or steroid-dependent cGVHD. This work has been supported by AIFA (Agenzia Italiana del FArmaco).

\section{0}

Circulating B-cell activating factor level predicts

likelihood of chronic GvHD flare and probability of successful steroid taper during extracorporeal photopheresis therapy

R. Whittle, H. Denney, A. Alfred, P.C. Taylor

Rotherham General Hospital (Rotherham, UK)

Introduction: Extracorporeal photopheresis (ECP) is an important second line therapeutic intervention in steroid refractory chronic GVHD (cGVHD) with recognised efficacy as a steroid sparing agent. Few reliable biomarkers predicting ECP response exist. B-cell activating factor (BAFF) has described roles in immature B-cell survival. Elevated BAFF levels reportedly correlate with cGVHD activity and excess BAFF may contribute to CGVHD maintenance. We report that BAFF level following 6 months of ECP therapy predicts the likelihood of disease flare and capacity for successful steroid taper.

Methods: We retrospectively evaluated 28 adult patients undergoing ECP for steroid-refractory, resistant or intolerant cGVHD. ECP was performed using the Therakos $\mathrm{XTS}^{\mathrm{TM}}$ or Cellex ${ }^{\mathrm{TM}}$ devices. ECP treatment schedule was 2-weekly dual treatments for an initial 3 months, then monthly paired treatments until at least 12 months. $24 / 28$ patients were receiving steroids at start of ECP. Skin disease response was assessed using the Modified Rodnan skin scoring system. Extracutaneous organ cGVHD response was assessed by reduction in symptoms as defined by NIH criteria. Disease flare was defined as significant symptomatic increase of, or (re)appearance of, GVHD in affected organ(s). Initiation of steroid or other immunosuppressives during treatment was also regarded as loss of disease control. Successful uninterrupted steroid taper was defined as the capacity for dose reduction without steroid re-escalation between 3 and 18 months of ECP. Soluble BAFF in patient sera was measured prior to ECP, and at 3, 6, 9 and 12 months of ECP using commercially available enzyme-linked immunosorbent assay.

Results: All patients with serum BAFF levels above $4 \mathrm{ng} / \mathrm{ml}$ following 6 months of ECP therapy $(n=15)$ experienced a loss of disease control as evidenced by GVHD flare between 3 and 18 months of ECP. This resulted in re-escalation of steroid dose in $13 / 15$ patients $(87 \%)$ and steroid introduction in 1 patient. Loss of GVHD control was significantly less common amongst patients with BAFF levels below $4 \mathrm{ng} / \mathrm{ml}$ at 6 months of ECP; $6 / 13$ patients experienced disease flare between 6 \& 18 months $(P=0.001$, Fishers exact) resulting in steroid or cyclosporine re-escalation in $5 / 13$ patients $(P=0.002)$ whilst steroid taper without escalation was possible in $7 / 11$ patients $(\mathrm{P}=0.001)$.

Conclusions: Our data supports further prospective studies to assess the potential prognostic value of early BAFF measurement in ECP therapy for CGVHD.

\section{1}

Rapamycin prevents experimental sclerodermatous chronic graft-versus-host disease in mice

L. Belle, M. Binsfeld, S. Dubois, M. Hannon, J. Caers, A. Briquet, C. Menten, Y. Beguin, S. Humblet-Baron, F. Baron University of Liège (Liège, BE)

Background: The most widely used mice model of chronic graftversus-host disease (cGvHD) is an MHC-matched bone marrow transplantation model of sclerodermatous CGvHD. A limitation of that model is that mortality is relatively low, making difficult to study the impact of potentially therapeutic compounds.

Aims: To develop a more severe model of cGVHD and to assess the impact of Rapamycin administration in that model.

Results: Lethally irradiated Balb/C mice were injected with $10 \times 10^{6}$ bone marrow cells and $70 \times 10^{6}$ splenocytes from B10.D2 donor mice. Twenty-one days later, all mice developed cGvHD. For the severe model, donor B10.D2 mice were injected with $0.5 \times 10^{6}$ splenocytes from Balb/C twenty-one days before 
transplantation. All mice from the severe model $(n=8)$ died a median of 32 days while 3 of 7 mice in the classical model survived beyond day 52 . Mean survival was decreased in the severe model compared to the classical model ( 32 days versus 37 days; $p=0.0185$ ). Recipient mice in the severe group experienced higher weight loss, hair loss and skin fibrosis. Numbers of T lymphocytes (231.9 \pm 151.4 versus $951 \pm 532.8 ; p=0.0032)$ and CD4+ $T$ cells $(63.25 \pm 41.93$ versus $135.0 \pm 14.39$; $p=0.0018$ ) per microliter of blood at day 21 were lower in the severe group than in the classical model. Moreover, number of regulatory $T$ cells (Tregs) was decreased in the severe model $(1.250 \pm 0.8864$ versus $8.000 \pm 6.753 ; p=0.0151)$.

We then investigated whether rapamycin administration could prevent GVHD in the severe model. All $(n=8)$ mice treated with PBS (placebo) died a median of 32 days after transplantation, while 6 of 8 mice given $1 \mathrm{mg} / \mathrm{kg} /$ day i.p. rapamycin survived beyond day $52(p=0.0012)$. Number of Tregs $/ \mu l$ was higher at day 21 in rapamycin-treated mice than in mice given PBS $(2.000 \pm 1.195$ versus $1.250 \pm 0.8864 ; p=0.0796)$. Moreover, number of naïve CD4+T $(10.00 \pm 4.192$ versus $30.25 \pm 5.185 ; p=0.0089)$ and effector memory T cells (EMT) $(30.67 \pm 3.180$ versus $67.33 \pm 7.881$; $\mathrm{p}=0.0125$ ) were higher in rapamycin mice. Finally, proliferation of EMT (assessed by flow cytometry using Ki-67) was higher in PBS than in rapamycin mice $(45.28 \% \pm 4.084$ versus $31.90 \% \pm$ 2.003; $p=0.0474$ ).

Conclusion: We have developed a mice model of severe cGVHD. Interestingly, rapamycin prevented death from cGVHD in that model, perhaps through in vivo expansion of Treg.

\section{Stem Cell Source and Donor}

\section{2}

Different effect of HLA disparity on transplant outcomes after single unit cord blood transplantation between paediatric and adult patients with leukaemia

Y. Atsuta, M. Takanashi, Y. Morishima, S. Taniguchi, S. Takahashi, T. Nagamura-Inoue, K. Kato, Y. Kanda on behalf of the HLA Working Group of the Japan Society for Hematopoietic Cell Transplantation

Recent advances in unrelated cord blood transplantation (UCBT) has provided increased chances for patients with hematological malignancies to receive hematopoietic stem cell transplantation (HSCT).

We have investigated the effect of HLA disparity of unrelated cord blood on HSCT outcome in children and in adults separately. 498 children aged 15 years or younger (HLA -A, -B low resolution and $-\mathrm{DRB} 1$ high resolution matched, $n=82$, one locus mismatched, $n=222$, two loci mismatched, $n=158$, three loci mismatched, $\mathrm{n}=36$ ) (median age, 5 years) and 1,880 adult patients (HLA matched, $n=71$, one locus mismatched, $n=309$, two loci mismatched, $n=1,025$, three loci mismatched, $n=475$ ) whose age was 16 years or older (median age, 49 years) at the time of transplant were analyzed. Subjects were recipients of single unit UCB as first HSCT with leukemia. Median infused total nucleated cell number was $5.30 \times 10^{7} / \mathrm{kg}$ in children and $2.52 \times 10^{7} / \mathrm{kg}$ in adults $(p<0.001)$.

With adjusted analyses, in children, HLA two-antigen mismatched UCBT showed significant increased risk of overall mortality (relative risk $[R R]=1.61, P=0.042$ ) and transplant-related mortality $(R R=3.55, P=0.005)$ compared to HLA matched. Risk of relapse did not differ significantly. Risk of mortality increased according to the number of mismatched loci ( $p$ for trend, 0.043 and 0.002 for overall mortality and TRM) The risk of relapse was not different among HLA disparity groups in children. Risk of grade 2 to 4 acute GVHD was increased in one- $(R R=2.18$, $\mathrm{P}=0.003)$ and two- $(\mathrm{RR}=2.51, \mathrm{P}=0.001)$ loci mismatched in children. Two-loci mismatched was associated with higher risk of grade 3 to 4 acute GVHD in children ( $R R=2.45, P=0.041)$.
In Adults, the risk of mortality did not increase with the number of mismatched loci $(R R=0.98, P=0.924$ for one-locus mismatched, $R R=0.88, P=0.423$ for two-loci mismatched, and $R R=0.95, P=0.746$ for three-loci mismatched for overall mortality). In adults, risk of relapse was significantly decreased in two- loci mismatched $(R R=0.67 P=0.029)$. Risk of $T R M$, grade 2 to 4 or grade 3 to 4 acute GVHD did not differ among HLA disparity groups in adults.

Effect of HLA disparity on transplant outcomes were different between children and adults. In children, increased number of mismatched HLA loci correlated with increased risk of mortality. In adults, there was no increase in mortality with increase in the number of mismatched HLA loci.

\section{3}

Co-infusion of haematopoietic progenitors from a HLA non-identical adult donor is a most efficient strategy for cord blood transplants early neutrophil recovery, engraftment and survival in adults with haematological malignancies

M.N. Fernandez (1), C Regidor (1), G Bautista (1), R Fores (1), I.I Sanjuan (1), J.A. Garcia-Marco (1), B. Navarro (1), I. Krsnik (1), E. Ojeda (1), S. Gil (1), A. de Laiglesia (1), I. Millan (1), M. Kwon (2), J. Gayoso (2), J.L. Diez-Martin (2), R. Duarte (3), J:R. Cabrera (1)

(1)Hospital Universitario Puerta de Hierro Majadahonda (Majadahonda, ES); (2)Hospital General Universitario Gregorio Marañon (Madrid, ES); (3)Hospital Duran i Reynals (Hospitalet, ES)

Late engraftment is a risk factor for CBT in adults. To overcome this limitation in 1999 we developed the "dual transplant" method (1): co-infusion of only one CB unit and highly T-depleted mobilized CD34+ (HP) cells from an adult third party donor (TPD), haploidentical or not. Here we report data from 98 adults $(61 / 37 \mathrm{M} / \mathrm{F})$ median weight $70 \mathrm{Kg}(42-111)$ transplanted in 3 Spanish centers to treat high risk hematological malignancies (HM). Indications: Acute Leukemia (AL) 85 (43 AML, 32 ALL), other 13.

Transplant products cellularity and HLA compatibility data shown in Table 1; 105 CB units were used as a 2nd unit was required for 7 patients: 2 rejections; 4 graft failures due to lack of viable HP; 1 relapse.

Conditioning: For 49: 10 Gy fractionated TBI, Fludarabine 60 $\mathrm{mg} / \mathrm{m}$, CTX $120 \mathrm{mg} / \mathrm{kg}$ and ATG; other 49 received Busulfan $3,2 \times 2-3 \mathrm{mg} / \mathrm{kg}$ instead of TBI.

Post-Tx treatment: G-CSF, Prednisone $1 \mathrm{mg} / \mathrm{Kg} 8-14$ days and CsA till full CB chimerism.

Engraftment data shown in Table 1. The TPD did not take in 8 cases: 1 due to very early CB engraftment; 6 seemingly due to recipient allosensitation against TPD (mother or husband). Other 6 who had engraftment failure/rejection of the initial CBT had sustained TPD graft until the take of a second CBT, given after 33-94 days preceded by a 2nd conditioning (Fludarabina + ATG \pm 2 Gy TBI or Thiotepa+Fludarbine+ATG). Morbi-mortality and survival data on incidence of TRM, relapses and GVHD are shown in Table 1. In no case were TPD cells involved in aGVHD lesions. Most common infections were CMV (72 episodes), declining after 3-4 months with 6 CMV-diseases causing 5 deaths. Other 2 deaths were due to toxoplasmosis and 1 each to EBV-PTLS, leishmaniasis and scedosporium prolificans infection (related to long pre-tx neutropenia).

Conclusions: Time to neutrophil recovery after dual transplants is consistently shorter than reported for other approaches (ex-vivo expansion, double CBT, intrabone infusion), resulting in low risk of early infections. This and the favorable data on aGVHD and relapses (i.e. GVT) contribute to less hospital days, what together with the procedure relative low cost translates into favorable costs/results ratio. The procedure may allow selection of CBT units prioritizing HLA compatibility to cell content and use of a fraction of CBT 
Table 1- Transplant, products, engraftment, GVHD, TRM and survival data

\begin{tabular}{|c|c|c|c|}
\hline Patients $=98 ; \mathrm{TPD}=98^{*} ; \mathrm{CB}$ units $=105^{\mathrm{kt}}$ & Median & (P25 - P75) & (P10-P90) \\
\hline CBT TNC cells/Kg x10^7 (pre-freeze) & 2.47 & $2.04-3.0$ & $1.64-3.44$ \\
\hline CBT CD34+ cells/Kg x 0^6 (pre-freeze) & 0.12 & $0.08-0.18$ & $0.05-0.29$ \\
\hline CBT CFU $\times 10^{\wedge} 4$ (post thaw) (range $\left.0-15.5\right)^{* \pi *}$ & 2,71 & & \\
\hline TPD CD34+ cells/ Kg x10^6 & 2,48 & $2.20-2.90$ & \\
\hline TPD CD 3+ cells/Kg x10 4 & 0.30 & $0.18-0.45$ & $0.09-0.82$ \\
\hline Days to ANC recovery $\left(>0.5 \times 10^{\mathrm{n} 9 / \mathrm{L})}\right.$ & 12 & 10-17 & $10-27$ \\
\hline Days to platelet recovery $\left(>20 \times 10^{n} 9 / L\right)$ & 38 & $27-58$ & $20-129$ \\
\hline Days to full CB chimerism & 48 & $29-85$ & $17-186$ \\
\hline
\end{tabular}

i Haphidentical relative, 74. Non-haphidentical (related or unrelated), 24

** HLA compatibility (A B antigen ic; DR allelic): $3 / 6,4 \% ; 4 / 6,50 \% ; 5 / 6,35 \% ; 6 / 6,11 \%$

** No CEUs in 4 units which did not engraft; patients received second CBT

\begin{tabular}{|c|c|}
\hline Follow up time of surviving patients: median (range) & 60 months (0.3-144) \\
\hline CI Patients reaching full CB chimerism ( $95 \%$ confidence interval) & $83.7 \%(76-91)$ \\
\hline $\mathrm{Cl}$ of TRM ( $95 \%$ confidence interval) & $35.6 \%(27-47)$ \\
\hline $\mathrm{Cl}$ of Relapse deaths ( $95 \%$ confidence interval) & $15.5 \%(9-26)$ \\
\hline $\mathrm{Cl}$ of agVYHD (all grades) & $54.4 \%$ \\
\hline $\mathrm{Cl}$ of aGyHD Grade II & $24.4 \%$ \\
\hline $\mathrm{Cl}$ of agVhHD Grades III-IV & $6.0 \%$ (4 deaths) \\
\hline CGyHD incidence (75 patients at risk) & $28.0 \%$ (6\% extensive) \\
\hline OS and DFS (K-M) (5-10 years), all patients & $47 \% \quad \& \quad 44 \%$ \\
\hline OS (K-M) (5-10 years), patients older than 50 years: & $39 \%$ \\
\hline OS and DFS (K-M) (5-10 years), AL patients transplanted in CR: & $57.5 \% \&$ \\
\hline
\end{tabular}

$P 25-P 75=$ interquartiles range (IQR); P10-P90= range between percentiles 10 and 90 of the $\mathrm{Cl}$ curve

$\mathrm{Cl}=$ Cumulative incidence. $\mathrm{CR}=$ complete remission. $\mathrm{K}-\mathrm{M}=$ Kaplan-Meier. $\mathrm{AL}=$ acute leukemia.

$O S=$ overall survival. DFS= disease free survival. $A N C=$ absolute neytrophil count

units of high cell content for ex vivo generation of effector T-cells that might be used to enhance immunological recovery. Dual transplant advantages have been confirmed by others (2-3).

References:

1. Fernandez MN, BJH, 2009;147:161-76.

2. Liu et al. Blood prepublished online October 5, 2011.

3. Gormley et al. ASH 2010. Abstract 2352.

\section{4}

Thymic reconstitution, chimerism development and survival after cord blood transplantation

J. Mattsson (1), S. Berglund (1), D. Sairafi (1), J. Gertow (2), P. Ljungman (3), M. Uzunel (2), J. Winiarski (4), O. Ringden (1), M. Remberger (1), M. Uhlin (2)

(1)Center for allogeneic stem cell transplantation (Stockholm, SE); (2)Clinical Immunology (Stockholm, SE); (3)Hematology (Stockholm, SE); (4)Pediatrics (Stockholm, SE)

Cord blood as a source of stem cells has been a successful addition to the field of allogeneic stem cell transplantation (SCT). The drawback is that patients suffer from a longer period of compromised immunity. We analyzed T cell Receptor Excision Circles (TRECs), Immunoglobulin G (IgG), immunoglobulin $\mathrm{M}(\mathrm{IgM})$ levels, lineage-specific chimerism analysis and patient outcome after cord blood transplantation (CBT) in 50 patients transplanted at our center. Engraftment of neutrophils was seen in $88 \%$ of the patients at a median time of
29 days (range 3-79). Complete donor chimerism (DC) within the CD19+, CD3+, and CD33+ cell lineages was seen in $74 \%$, $72 \%$, and $76 \%$ of the patients, respectively. DC was associated with acute graft-versus-host disease (GVHD) grades II-IV for the CD3+ cell lineage $(p=0.01)$ and, in multivariate analysis, with total body irradiation (TBI) for all cell lineages $(p<0.01)$. Overall survival (OS) at one and five years was $55 \%$ and $43 \%$. Non-malignant diseases was associated with better 5 -year OS $(72 \%)$ than malignancies $(28 \%, p=0.026)$. In multivariate analysis, a negative correlation was seen between $\mathrm{OS}$ and age, hazard ratio (HR) $1.04 \quad(95 \% \mathrm{Cl}$ : $1.02-$ 1.06, p<0.001); acute GVHD grades III-IV, HR $3.43(95 \% \mathrm{Cl}$ : $1.95-6.02, p<0.001)$; and mesenchymal stem cell treatment $2.66(95 \% \mathrm{Cl}: 1.11-6.35, p=0.027)$. Transplant-related mortality (TRM) at 100 days and one year was $16 \%$ and $30 \%$. The incidence of acute GVHD grades II-IV was $34 \%$. Acute GVHD grades III-IV was associated with $A B 0$ incompatibility (HR 2.61, $\mathrm{p}=0.05)$ and myeloablative conditioning (HR 4.17, $p=0.047$ ). We found that TREC levels after CBT were lower in adults, patients with malignant disease, patients with myeloablative conditioning, and in patients with a lower nucleated cell dose in the graft. In addition mesenchymal stem cells (MSC) as co-infusion at the time of CBT had a negative effect on TREC reconstitution which may be one explanation for the decreased survival in MSC treated patients. Reduced IgM and IgG levels post CBT were associated with older patient age, a major $\mathrm{ABO}$ mismatch, and infusion of mesenchymal stem cells. Our results highlight the importance of close monitoring of the reconstitution of the immune system after cord blood 
transplantation. In addition it shows a potentially new suppressive effect of MSCs on the immune system.

\section{5}

Allogeneic peripheral blood stem cell transplantation from HLA-identical siblings versus allelic-matched unrelated donors in patients with acute myeloid leukaemia and myelodysplasic syndrome after reduced-intensity conditioning

M. Robin (1), R. Porcher (1), L. Adès (2), E. Raffoux (1), N. Boissel (1), J. Larghero (1), C. Himberlin (3), C. Gardin (2), A. Delmer (3), P. Fenaux (2), H. Dombret (1), G. Socié (1), R. Peffault de Latour (1)

(1)Hôpital Saint-Louis - APHP (Paris, FR); (2)Hôpital Avicenne APHP (Bobigny, FR); (3)CHU (Reims, FR)

The impacts of allelic HLA matching in patients (pts) with AML and MDS who receive PBSC after a RIC remain to be addressed. In this study, we aim to compare the impact of the donor type in this setting: HLA identical sibling (S) versus high resolution HLA matched 10/10 unrelated donor (MUD). From 01/01 to 12/10, 108 pts with AML $(n=63)$ and MDS $(n=45)$ received PBSC after RIC in our center, either from S $(n=69)$ or MUD $(n=39)$. RIC was fludarabine based in $95 \%$ of pts. Engraftment, acute and chronic GvHD, transplantation-related mortality (TRM), relapse rate $(R R)$ and overall survival (OS) at 3 years were compared according to type of donor: S or MUD. WHO classification for MDS was RAEB1 (24\%), RAEB2 (36\%), MDS transformed into secondary AML $(20 \%)$, CMML2 $(9 \%)$, RA $(4 \%)$, or other $(7 \%)$. Disease risk was assumed by cytogenetic (MRC for AML, IPSS for MDS) and EBMT score (good risk: CR1 for AML or MDS or untreated MDS, intermediate risk: CR2 for AML, CR2 or partial remission for MDS, poor risk: all other status). Cytogenetic was poor, intermediate or good for 21,74 and $5 \%$ of AML and 24 36 and $40 \%$ of MDS, respectively. EBMT score at time of HSCT was poor, intermediate or good for $29,7,64 \%$ of MDS and 11 , $21,68 \%$ of AML, respectively. Pts characteristics according to type of donor were similar for age (median 57 years), gender and disease distribution. Particularly, disease risks were comparable in 2 groups. Conversely, conditioning regimen (more ATG in MUD), donor age (younger for MUD) and number of CD34+ cells infused (higher in MUD) were different. All pts engrafted. The cumulative incidence of acute GvHD was $40 \%$ with $S$ and $44 \%$ for MUD $(p=0.58)$. The cumulative incidence of chronic GvHD was $49 \%$ with S and $45 \%$ with MUD ( $p=0.66)$. No significant risk factor was associated with acute or chronic GvHD. TRM was $17 \%$ and $22 \%$ with S and MUD, respectively $(p=0.55)$. Adjusting for age, MDS was the only factor increasing TRM (HR $3.4 ; \quad p=0.02$ ). RR was $46 \%$ with $S$ and $30 \%$ with

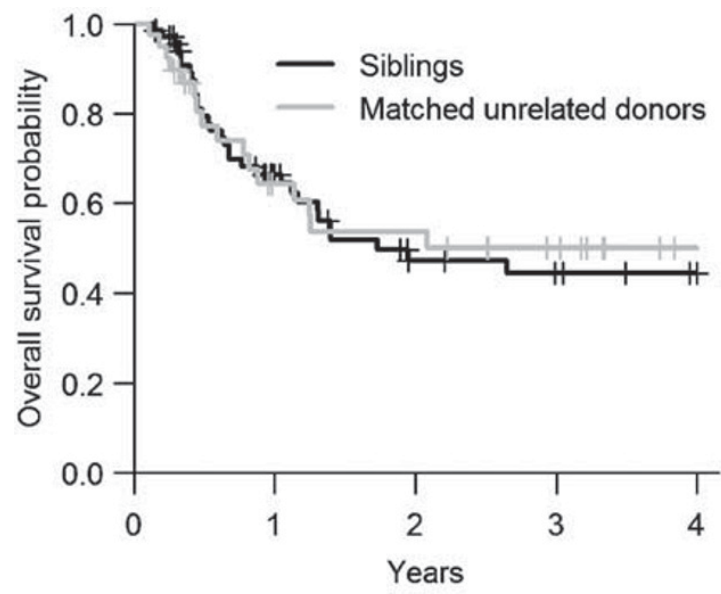

Figure 1: Overall survival according to donor type
MUD ( $p=0.28)$. OS was $44 \%$ with S $(95 \% \mathrm{Cl}$ : $33-61)$ and $50 \%$ with MUD (95\% Cl: 35-71) (Figure 1). Cytogenetic and EBMT score did not significantly influence the OS. After adjustment for age, cytogenetic risk, ATG and number of CD34+ cells infused, donor type still did not influence OS. In an homogeneous group of pts with AML or MDS receiving PBSC after RIC, survival was strictly identical if the donor was an HLA matched sibling donor or MUD including after adjustment for variables potentially impacting survival.

\section{6}

Impact of donor specific anti-HLA antibodies on graft failure and survival after cord blood transplantation with reduced-intensity conditioning regimen. A Eurocord, SFGM-TC and SFHI analysis

A. Ruggeri (1), V. Rocha (1), E. Masson (2), R. Cunha (1), L. Absi (3), A. Boudifa (4), B. Coeffic (5), D. Charron (2), A. Devys (6), M. De Mattei (7), V. Dubois (8), D. Hanau (9), F. Hau (10), I. Jollet (11), D. Masson (12), B. Pedron (13), P. Perrier (14), E. Gluckman (1), P. Loiseau (2)

(1)Eurocord (Paris, FR); (2)Hôpital Saint Louis (Paris, FR); (3) TC (St Etienne, FR); (4)Hôpital Pitié Salpetriere (Paris, FR); (5)TC (Angers, FR); (6)TC (Nantes, FR); (7)TC (Nice, FR); (8)TC (Lyon, FR); (9)TC (Strasbourg, FR); (10)TC (Normandie, FR); (11)TC (Poitiers, FR); (12)TC (Grenoble, FR); (13)Hôpital Robert Debré (Paris, FR); (14)TC (Nancy, FR)

Delayed hematopoietic recovery and graft failure (GF) are critical complications of cord blood transplantation (CBT) and are associated with TNC or CD34 cell dose and HLA disparities. Other factors such as patients's HLA-antibodies (Ab) may impact neutrophil recovery. To analyze the effect of anti-HLA$A b$ on CBT outcomes we analyzed 206 pts who underwent CBT after RIC regimen from 2000-2010. Median follow-up was 36 months. Eighty-two percent of pts were transplanted for malignancies, $40 \%$ had single and $60 \%$ double-CBT. Thirty percent of CBU had 0-1 HLA mismatch (A, B, DRB1), 67\% received CyFluTBI2Gy and median infused TNC was $3.7 \times 10^{7} / \mathrm{Kg}$. Pre-transplant serum was tested for HLA-Ab with a panel of fluorescent beads coated with single HLA-antigen using LuminexTM platform. Results were interpreted as fluorescence intensity (MFI) against donor-specific mismatch. Overall 48 pts $(23 \%)$ had anti-HLA-Ab before CBT and those were donor specific anti-HLA-Ab (DSA) in 16 pts. Among the 16 pts with DSA (11 females, 5 males), 9 had single and 7 double-CBT (none had DSA directed to both CB units). Seven pts had DSA vs to HLA-Class-I, 5 vs to HLA-Class-II and 4 to both HLAClass-I and-II. DSA threshold ranged from 1620-17629 MFI. Cumulative incidence $(\mathrm{Cl})$ of day-60 neutrophil engraftment was $76 \%$. It was $44 \%$ for recipients with DSA and $81 \%$ in pts without DSA $(p=0.006)$. There was no difference for pts with anti-HLA-Ab non donor-specific $(77 \%$ vs $69 \%)$. Multivariate model showed DSA (RR 2.7, $p=0.01$ ) and CBT before 2008 (RR 1.49, $p=0.03$ ) independently associated with GF. Seven pts with DSA engrafted, 4 after double-CBT and chimerism analysis showed the engraftment of the CBU with DSA in 1 case. Among 50 pts who failed engraftment,, $9(20 \%)$ pts had DSA specific for donor HLA-Class-I $(n=4)$ or Class-II $(n=2)$ or both Class-I and Class-II $(n=3)$. Cl of platelet recovery at day- 180 was $62 \%, 12$ of 16 patients with DSA did not achieve platelet recovery. Cl of 1-year TRM was $35 \%$. DSA was associated with higher TRM $(p=0.002)$. Overall survival at 3 -years was $44 \%$, it was $41 \%$ and $45 \%$ for pts with non-malignant and malignant disease respectively. OS was $47 \%$ for recipients without DSA and $25 \%$ for those with DSA, $p=0.006$. In multivariate analysis, the absence of DSA was the only factor associated with better survival (RR 2.41, $p=0.005$ ) Donorspecific anti-HLA-Ab in recipients of CBT is associated with failed engraftment and lower survival. Screening for DSA may be included in the algorithm of donor choice. 


\section{7}

Factors associated with race and CT-stage decision in a racially diverse sample of NMDP registrants

G. Switzer (1), M.A. Dew (1), J. Bruce (1), L. Myaskovsky (1), D. Confer (2), L. Abress (2), A. DiMartini (1), D. Harrington (1), S. Ohngemach (1)

(1)University of Pittsburgh (Pittsburgh, US); (2)National Marrow

Donor Program (Minneapolis, US)

Rationale: The NMDP manages the largest international registry of volunteers registered to donate stem cells to unrelated patients with blood-related diseases. Key stages leading to donation include joining the registry, confirmatory typing (CT), and final work up. CT-stage has emerged as a critical decisionpoint in the process because most donor attrition occurs at this point. In addition, although more than $65 \%$ of whites continue toward donation at CT-stage, only $40 \%$ of members of all other ethnic groups continue. Higher attrition among ethnic minority donors disproportionately disadvantages ethnic minority patients searching for donors. Our goal was to examine characteristics of potential donors that might be associated with CT-stage decision and/or race/ethnicity.

Methods: We conducted telephone interviews with a stratified random sample of registry members who had initially matched a patient, were contacted at CT-stage, and who decided either to continue toward donation $(\mathrm{N}=843)$ or to opt-out of the registry $(\mathrm{N}=224)$. Measures included demographics, culturallyrelated variables (e.g., medical mistrust, religious objections to donation), psychosocial variables (e.g., emotional distress, self-esteem) and donation-related variables (e.g., ambivalence about donation, donation-related concerns). Analyses examined differences in these variables by CT-stage decision and racial/ethnic group.

Results: Those who opted-out of the registry at CT-stage had more religious objections to donation $(F=51.9, p<.001)$, more mistrust of the medical system $(F=5.0, p<.05)$, less trust that the stem cells would be used appropriately $(F=32.1, p<.001)$ more emotional distress $(F=29.6, p<.001)$, more ambivalence $(F=430.3, p<.001)$, and more medical concerns $(F=56.4, p<.001)$. Ethnic group analyses indicated that whites, compared to minority groups, had fewer religious objections to donation $(t=2.4$, $p<.05)$, less mistrust of the medical system $(t=2.5, p<.05)$, and more trust that the stem cells would be used appropriately $(\mathrm{t}=4.2, \mathrm{p}<.001)$. Asian Pacific Islanders had more emotional distress $(t=2.7, p<.01)$ and were more ambivalent about donation $(t=2.1, p<.05)$ than other groups.

Conclusions: These findings have important implications for the management of donors, particularly donors belonging to ethnic minority groups. They suggest that communications with donors at key contact points including recruitment and CT-stage could be tailored to address concerns specific to each group.

\section{O328}

Donation of bone marrow is associated with a higher rate of subsequent HPC donation in unrelated donors compared to PBSC

R. Lown (1), S. Tulpule (1), C.F. Craddock (2), J.A. Madrigal (1), N.H. Russell (3), R. Roest (1), B.E. Shaw (1)

(1)Anthony Nolan (London, UK); (2)Queen Elizabeth Hospital (Birmingham, UK); (3)Nottingham University Hospital, City Campus (Nottingham, UK)

Approximately 1 in 15 patients will undergo a second allogeneic haematopoietic cell (HPC) transplant, the majority using the same donor. Anthony Nolan carried out a retrospective study of subsequent HPC donations made by its donors over a sixyear period, from 2005 to 2011 , with the aim of predicting which donors were more likely to be called for a second donation. Methods: The initial transplants of those patients requiring a subsequent donation were examined: five key donor characteristics (route of harvest, HLA match, age, gender and CMV status) were analysed, and their incidence compared to that found in all HPC donations provided in the same period.

Results: 2105 HPC donations were made during this time, of which $117(5.6 \%)$ were subsequent donations. 93 (79.5\%) of these were to the same patient and the following results are for this group. The median time between donations was 198 days (range 39-4016 days). Main disease categories included acute myeloid leukemia $(28 \%, n=26)$, acute lymphoblastic leukemia $(14 \%, n=13)$, myelodysplasia $(12.9 \%, n=12)$, non-Hodgkin lymphoma $(10.8 \%, n=10)$, aplastic anaemia $(8.5 \%, n=8)$ and chronic myeloid leukaemia $(7 \%, \mathrm{n}=7)$ - this composition differed significantly from the overall sample (Chi-square goodness of fit test, $\mathrm{p}<0.001)$. Indications for second allogeneic transplant included primary graft failure (PGF, $7.4 \%, n=7$ ), secondary graft failure (SGF, $58.1 \%, n=54$ ), disease relapse (REL, $31.2 \%$, $n=29)$ and others $(3.2 \%, n=3)$. Mean cell doses at initial transplant were $2.88 \times 10^{8} / \mathrm{kg}$ total nucleated cells for bone marrow and $7.7 \times 10^{6} / \mathrm{kg}$ CD34+ cells for peripheral blood stem cells (PBSC). Bone marrow was more common as the source of stem cells at initial transplant in PGF $(71.4 \%, p=.012)$, SGF $(50 \%, p<0.001)$ and REL $(48.2 \%, p=0.005)$ when compared to the background frequency ( $24.6 \%$, weighted mean). There was a trend towards a greater frequency of HLA mismatched donors in the PGF group only $(p=0.093)$. Donor age $>30$, CMV positivity and female gender were not found to be more frequent. Conclusion: Although small, this study suggests that the donation of bone marrow confers a greater likelihood of a donor being called for a second HPC donation to the same patient when compared to PBSC collection $(8.01 \%$ vs $2.3 \%$, p < 0.001$)$. These findings have implications not only for harvest physicians when consenting donors for bone marrow harvest, but also for transplant centres when considering the most appropriate HPC source for their patients.

\section{Non-malignant Diseases}

\section{9}

Favourable outcome after reduced-toxicity conditioning with high-dose fludarabine, serotherapy and low-dosel targeted busulfan in 74 children and adults with non-malignant diseases

T. Güngör, M. Albert, M. Slatter, G. Stüssi, P. Teira, P. Stepensky, C. Vermont, I. Ahmad, P. Shaw, D. Moshous, A. Waver, P. Schlegel, C.-M. Farber, P. Veys, J. Fernandes, M. Telles da Cunha, S. Lachance, A. Fischer, R. Bredius, I. Resnik, B. Belohradsky, A. Gennery, A. Cant, U. Schanz, E. Haddad, R. Seger, K. Rentsch, A. Fasth, M. Hassan on behalf of the WP Inborn Errors

Bu/CY-based conditioning for HSCT may induce short term toxicity, e.g. VOD and long-term sequelae, e.g. infertility. Reduced toxicity conditioning regimens providing both sufficient myeloid donor engraftment and reduced toxicity are currently investigated.

58 pediatric $(0.3-17 \mathrm{ys})$ and 16 adult (18-39ys) patients $(n=55$ male; $n=19$ female) with non-malignant diseases (CGD $n=45$, SCID n=8, HLH n=3; WAS n=3; XLP/CID n=4, NEMO/OP n=2, Fucosidosis/ALD $n=4$, Thalassemia $n=3$, refractory anemia/ $\mathrm{PNH} n=2$ ) from 15 centers underwent conditioning comprising $180 \mathrm{mg} / \mathrm{sqm}$ fludarabine (d-8 to -3), low dose or targeted busulfan (d-5 to -2) and serotherapy with rabbit ATG (Fresenius: 40 $\mathrm{mg} / \mathrm{kg}$ or Genzyme: $7.5 \mathrm{mg} / \mathrm{kg}$ ) in MSD or unrelated CBT or alemtuzumab (0.5-0.6 mg/kg; $\mathrm{d}-8$ to -6$)$ in MUD/MUD HSCT. HSCT was performed at a median age 8.75 years (0.3-39 ys) using MSD $(n=29), \operatorname{MRD}(n=4)$, MUD/MMUD $(n=40)$ and haploidentical $(n=1)$ donors. BM $(n=59), \operatorname{PBSC}(n=11)$ and CB $(n=4)$ served as stem cell sources. GVHD-prophylaxis comprised CSA (until $d+160$ ) and mainly MMF (until $d+100$ ). Pharmakokinetic monitoring for busulfan was performed in 52 patients 
targeting at a submyeloablative AUC of $45-65 \mathrm{mg} / \mathrm{Lxh}$ for patients with PID and refractory cytopenias and a myeloblative AUC of 75-95 mg/Lxh for hemoglobinopathy, metabolic disease, WAS and CGD/MDS after gene therapy. In 22 patients where PK monitoring was not feasible reduced dose $(8 \mathrm{mg} / \mathrm{kg}$ orally or $6.4 \mathrm{mg} / \mathrm{kg}$ iv.; $\mathrm{n}=16)$ or full dose busulfan $(16 \mathrm{mg} / \mathrm{kg}$; $\mathrm{n}=6$ ) was administered.

$\mathrm{N}=5$ case of mild VOD responding well to therapy were observed. The rate of aGVHD grade III-IV and limited CGvHD was $4 \%$ (4 of 74 ) and $13 \%$ (10 of 74 ), respectively.

Neutrophil/platelet engraftment occurred in 71/74 (96\%) patients after a median of +19 and +22 days, respectively. 4 patients had autologous reconstitution/non-engraftment and were successfully retransplanted. One patient received a successful stem cell top-up due to prolonged pancytopenia. After a median follow-up of 15 months (range 4-102 mo), the overall and event free survival rates are $95 \%(70 / 74)$ and $89 \%$ (66/74), respectively. All surviving patients exhibit a $>90 \%$ myeloid donor chimerism except one OP patient with stable $70 \%$ myeloid chimerism. This protocol provides excellent short term toxicity and myloid engraftment features with high cure rates. Targeting busulfan administration is of major importance to steer therapy and to ensure myloid engraftment while reducing short- and long-term toxicity.

\section{0}

HLA-haploidentical stem cell transplantation in osteopetrosis - improved success rate despite high rejection risk

A. Schulz, D. Moshous, P. Stepensky, A. Fasth, A. Villa, G. Lahr, M. Cavazzana-Calvo, C. Schuetz, M. Hoenig, A. Fischer on behalf of the Inborn Errors Working Party

Infantile osteopetrosis is a rare heterogenous genetic disorder caused by mutations in one of several genes, which impair osteoclast function. Haematopoietic stem cell transplantation (HSCT) is the only treatment that can improve the phenotype. In patients without an HLA-matched family or unrelated donor, HLA-haploidentical HSCT has been performed with success rates between $24 \%$ and about $60 \%$. Here we report on a recent survey of HLA-haploidentical transplantation in patients with osteopetrosis.

Twenty-one patients with infantile osteopetrosis were transplanted at the University Hospitals of Ulm, Paris, Goteborg and Jerusalem between 2001 and 2009. DNA analysis revealed biallelic mutations in the TCIRG1 gene $(n=16)$, and the CLCN7 gene $(n=4)$, respectively. In one patient no causative mutation could be detected (genes sequenced: TCIRG1, CLCN7 RANK, RANKL, OSTM1). The age at transplant was 2 to 72 (median $15)$ months. The conditioning regimen was based on busulfan and fludarabin in all patients combined with cyclophosphamide in 7 and thiotepa in 14 patients, respectively. ATG or Campath$1 \mathrm{H}$ was used as prophylaxis for rejection and graft-versus-host disease. G-CSF mobilized cells from a parent were used as stem cell source after CD34 positive selection. Toxicity of the conditioning regimen was high with severe VOD or complications making ICU treatment necessary in 13/21 patients (62). Eleven patients $(52 \%)$ rejected their first graft and/or showed an autologous reconstitution after prolonged cytopenia. The risk for rejection or autologous reconstitution was associated with age older than 10 months (7/8 patients rejected) and less than $10 \times 10^{6} \mathrm{CD} 34+$ cells/kg in the graft (5/5 patients did not engraft). However, 8 of 11 patients could be rescued by a second HSCT after rejection and survived despite severe complications and prolonged aplasia. Notably, three patients aged 72, 42 and 16 months at their first HSCT died because of graft failure despite repeated transplantations. Eighteen of 21 patients (86\%) survived with a median follow up of 72 months (3-161 months)

These data indicate that HLA-haploidentical HSCT in osteopetrosis is an approach associated with severe complications, in particular a high risk for rejection in patients beyond the age of
10 months, but can be performed with an improved and quite high success rate compared to previous studies.

\section{1}

Outcome of unrelated donor bone marrow transplantation for patients with Thalassaemia major

F. Locatelli (1), R. Littera (2), D. Pagliara (1), B. Contoli (1), G. Giorgiani (3), M.E. Bernardo (1), A. Mastronuzzi (1), E. Piras (2), A. Vacca (2), R.M. Pinto (4), C. Giardini (5), G. Palumbo (1), G. La Nasa (2)

(1)IRCCS Bambino Gesu' Children's Hospital (Rome, IT); (2)Ospedale R. Binaghi (Cagliari, IT); (3)Fondazione IRCCS Policlinico S. Matteo (Pavia, IT); (4) IRCCS Bambino Gesu' Children's Hospital (Rome, IT); (5)Ospedale San Salvatore (Pesaro, IT)

Allogeneic BMT still remains the only treatment potentially able to definitively cure thalassemia major. We evaluated the outcome of patients transplanted from an unrelated donor (UD), selected using stringent criteria of compatibility after high-resolution molecular typing of HLA class I/II loci, analyzing factors with a prognostic relevance. We studied 122 patients (96 children and 26 adults, $71 \mathrm{M} / 51 \mathrm{~F}$, age range at time of BMT 1-35 years, median 10.5). 15 patients had positive HCV serology. 39 pediatric patients belonged to the class I of risk of the Pesaro classification, 41 to the class II and 16 to the class III. 106 donor/recipient pairs were matched for the HLA-A,-B,-C,-DRB1 loci; 16 donor/recipient pairs had a single HLA-class I allelic disparity. Patients received a myeloablative regimen based on busulfan/treosulfan, thiotepa and either cyclophosphamide (54 patients), or fludarabine (68 patients). All patients received fresh, unmanipulated BM cells (median dose of nucleated cells infused: $5 \times 10^{8} / \mathrm{kg}$; range $\left.1.4-15\right)$. GvHD prophylaxis was homogeneous in all patients and consisted of CsA, short-term MTX and ATG (2.5 mg/kg from day -4 to -2$) .50$ patients at risk (28\%) developed grade II-IV acute GvHD with 16 of them $(13 \%)$ experiencing grade III-IV acute GvHD. 14 patients at risk (13\%) had chronic GVHD, which was limited in 8 cases and extensive in 6.20 patients died and 16 patients had either primary (11 patients) or secondary (5 patients) graft failure. Acute and chronic GvHD were the main causes of death accounting for 6 and 4 fatal events respectively; graft failure was responsible of 2 fatal events. The 5 -year OS and thalassemia-free survival (TFS) were $84 \%(95 \% \mathrm{Cl}, 76-90)$ and $75 \%(95 \% \mathrm{Cl}, 66-81)$ respectively. The OS of children belonging to class I, II and III of the Pesaro classification was $97 \%(95 \% \mathrm{Cl}, 83-99), 85 \%$ $(95 \% \mathrm{Cl}, 70-93)$ and $81 \%(95 \% \mathrm{Cl}, 51-93)$, respectively, while that of adults was $65 \%(95 \% \mathrm{Cl}, 44-80)(p<0.01)$. In multivariate analysis, only occurrence of grade III-IV acute GvHD and older patient's age predicted a poor outcome (HR 7.02, 95\% Cl $2.76-17.83, p=0.001$ and $1.10,95 \% \mathrm{Cl} 1.03-1.18$, respectively). These data indicate that, provided that selection of the donor is based on stringent criteria of HLA-compatibility, transplantation from UD is able to cure a large proportion of thalassemia major patients. Refinements of strategies aimed at preventing both GvHD and graft failure could further improve the chance of cure of these patients.

\section{2}

Outcome of haematopoietic stem cell transplantation in patients with chronic granulomatous disease at a national centre

J. Lane (1), M. Slatter (1), Z. Nademi (2), P. Tierney (2), D. Barge (1), S. Hambleton (1), T. Flood (2), A. Cant (2), M. Abinun (2), G. Jackson (1), M. Collin (1), A. Gennery (1) (1)Newcastle University (Newcastle upon Tyne, UK); (2)Newcastle upon Tyne Hospital NHS Foundation Trust (Newcastle upon Tyne, UK)

Introduction: Chronic Granulomatous Disease (CGD) is a rare X-linked or autosomal recessive inherited primary immuno- 
deficiency leading to multiple opportunistic infections, abscesses and inflammation. $55 \%$ survive to 30 years old. HSCT offers curative treatment in patients with severe and life threatening complications from the disease.

Methods: Retrospective analysis of case notes of all CGD patients who underwent HSCT in Newcastle Upon Tyne Hospitals NHS Trust.

Results: 41 patients have undergone HSCT for CGD since 1997; 35 survived and 6 died (1 during conditioning). Median age at diagnosis and HSCT were 3.21 (range $0-16$ ) and 8.38 (range $0.75-27.2$ ) years respectively. 3 patients were adults (>18 years) at time of transplant. 2 of 6 patients who died had active fungal infection at time of transplant and 1 other died from pulmonary haemorrhage following aspergilloma. Median age at HSCT of those who survived was 7.17 years (range 0.75-16.9) and those who died was 18 years (range 8.33-27.2). 39 patients had had an infection prior to transplant, the commonest being pneumonia or lung abscesses, lymphadenitis and perianal or gluteal abscesses. Aspergillus spp. were the most common organisms isolated, followed by Staphylococcus aureus. 26 patients had CDG-associated colitis prior to transplantation. 40 patients received HSCT, 2 underwent a further transplant due to graft failure and 3 required top-up stem cell transfusions. 15 patients had GvHD, 15 skin (I-II), 4 liver and 2 gut. 6 patients developed chronic GvHD, 1 of whom died. 2 remain on immunosuppression. 1 patient had recurrence of colitis. 28 patients have normal neutrophil oxidative burst (NOB; $>95 \%$ ) after transplant, 6 have a dual population of neutrophils but are clinically normal and 1 patient has $<20 \%$ NOB and is awaiting further HSCT 6 years after umbilical cord stem cell transplant. All patients with greater than 2 years follow up have shown a response to primary childhood vaccinations. None require antifungal prophylaxis and 2 are on long term antibiotic prophylaxis.

Conclusions: HSCT is curative for CGD with $85 \%$ survival in this cohort and good long term graft function, restoring neutrophil function and responding to vaccination. Inflammatory colitis also resolves post transplant. Survival is related to severity of complications pre-HSCT and hence age at HSCT. HSCT should be considered in the early management of CGD, particularly when there is a well matched donor.

\section{3}

Successful treatment of DOCK8 hyper IgE syndrome by haematopoetic stem cell transplantation

H. Boztug (1), C. Karitnig-Weiß (2), B. Ausserer (2), E. Renner (3), M. Albert (3), J. Sawalle-Belohradsky (3), G. Mann (1), C. Peters (1), A. Lawitschka (1), S. Matthes-Martin (1)

(1)St. Anna Kinderspital (Vienna, AT); (2)Krankenhaus Dornbirn (Dornbirn, AT); (3)Dr. von Haunersches Kinderspital, LMU (Munich, DE)

Hyper IgE Syndrome (HIES) constitute a rare subset of immunodeficiencies collectively characterized by a trias of increased serum IgE levels, eczematous rash and recurrent skin and pulmonary infections. Autosomal recessive HIES has been found to be associated with mutations of DOCK8. Hitherto, few reports have been published on patients with DOCK8 HIES treated with hematopoetic stem cell transplantation (HSCT). We report about a girl born to non-consanguineous parents who presented with a severe generalized refractory eczema beginning in the first month of life, multiple food allergies, recurrent sinopulmonary infections, chronic mollusca contagiosa and a chronic HSV1 infection of the eye. Extremely high IgE levels of $>30.000 \mathrm{IU} / \mathrm{ml}$ and high values for absolute eosinophils were found. A homozygous mutation in DOCK8 (c.850_851delCT; p.L284fs X292) was identified. Examinations before HSCT revealed a solitary lung nodule. Histology and culture after surgical resection showed aspergillus fumigatus. Following a reduced intensity conditioning regimen consisting of fludarabine, thiotepa, melphalan and ATG the child received bone marrow stem cells $\left(4,05 \times 10^{6} / \mathrm{kg}\right.$ CD34+) from a $10 / 10$ matched
HLA identical unrelated donor at the age of 3 years. Immunosupression included cyclosporin and mycofenolatmofetil (until day +30 ). Engraftment with absolute neutrophil counts $>500 / \mu \mathrm{l}$ occurred on day +19 . Transplant complications included acute skin GvHD grade II at day +26 which responded well to systemic steroids as well as a CMV pneumonitis 4 weeks after SCT which was treated with ganciclovir. Soon after transplantation, the eczematous rash and the HSV 1 infection of the eye disappeared, while the chronic mollusca contagiosa infection persisted for several months. Peripheral blood consistently showed $>99 \%$ donor chimerism in all cell subsets. Cyclosporine was discontinued at day +180 . Six months after transplantation the child is in very good health without signs of infection. The skin appears almost unremarkable with only small remnants of molluscum lesions. Food allergies nearly completely disappeared. IgE levels and absolute numbers of eosinophils have normalized.

In conclusion, we have shown in a patient that DOCK8 hyper IgE syndrome can be treated successfully with HSCT. To more comprehensively evaluate the risk and benefit of HSCT in DOCK8 deficiency a study including HSCT centers worldwide is underway registered at the European Society of Immunodeficiencies (ESID).

\section{4}

Wiskott-Aldrich syndrome presenting with a clinical picture resembling juvenile myelomonocytic leukaemia A. Yoshimi (1), Y. Kamachi (2), K. Imai (3), T. Morio (3), N. Watanabe (4), A. Konoshita (5), N. Nakadate (6), K. Tamura (7), S. Oozono (8), R. Kobayashi (9), H. Kigasawa (10), M. Jakob (11), S. Nonoyama (12), A. Manabe (13), C. Niemeyer (1), S. Kojima (2)

(1)University Children's Hospital (Freiburg, DE); (2)Nagoya University Graduate School of Medicine (Nagoya, JP); (3)Tokyo Medical and Dental University Graduate School of Medical and Dental Sciences (Tokyo, JP); (4)Children's Medical Center, Japanese Red Cross Nagoya First Hospital (Nagoya, JP); (5)St. Marianna University School of Medicine (Kawasaki, JP); (6)Kitasato University School of Medicine (Sagamihara, JP); (7)Gunma University Graduate School of Medicine (Gunma, JP); (8)Kurume University School of Medicine (Kurume, JP); (9)Sapporo Hokuyu Hospital (Sapporo, JP); (10)Kanagawa Children's Medical Center (Kanagawa, JP); (11)University of Regensburg (Regensburg, JP); (12)National Defense Medical College (Tokorozawa, JP); (13)St. Luke's International Hospital (Tokyo, JP)

Background: Wiskott-Aldrich syndrome (WAS) is a rare X-linked immunodeficiency caused by genetic defects of the WAS protein (WASP) gene. Patients with WAS typically demonstrate micro-thrombocytopeniain the absence of other hematological symptoms. Here we report on 6 male infants with WAS, presenting with an unusual complication, which was initially undistinguishable from juvenile myelomonocytic leukemia (JMML).

Methods: The central morphological and laboratory diagnosis of JMML has been performed by the Japanese Society of Pediatric Hematology (JSPS) and the European Working Group of MDS in Childhood (EWOG-MDS) in Japan and Europe, respectively. The laboratory diagnosis of JMML consisted of a mutational analysis of genes of the RAS-signaling pathway including PTPN11, KRAS, NRAS $(n=6)$ and $c-C B L(n=1)$. The diagnosis of WAS was made by the analysis of the intracellular WASP expression by Western blot analysis or flow cytometric analysis and a detection of WASP gene mutation.

Results: Five Japanese patients and one German patient with an initial suspected diagnosis of JMML were later diagnosed with WAS. Leukocytosis, monocytosis, presence of myeloblasts and hematopoietic precursor cells in peripheral blood and a hypercellular bone marrow with dysplasia were first observed at the median age of 2 months (1-3 months) and persisted for several months. The morphological and hematological pictures are compatible with the diagnosis of JMML. 
All patients demonstrated thrombocytopenia since birth and bloody stool noted first soon after birth; the later is typical for WAS but not for JMML. Splenomegaly was observed in one patient only; absence of splenomegaly at diagnosis is highly unusual for JMML. A mutational analysis of genes of the RASsignaling pathway was negative in all 6 patients. Non-hematological features such as eczema and bloody stool finally led to the diagnosis of WAS at the median age of 4 months ( 3 to 9 months); in all caes the diagnosis was confirmed by the lack of intracellular WASP expression and a detection of WASP gene mutation. Interestingly, mean platelet volume was normal in these patients and 3 of them demonstrated occasional "giant" platelets, which made the straightforward diagnosis of WAS difficult.

Conclusions: WAS should be considered in male infants presenting with JMML-like features if no molecular markers of JMML can be demonstrated. Clinical information such as bloody stool are helpful for the early consideration of WAS.

\section{5}

Guideline on infection prevention in paediatric allogeneic stem cell transplantation recipients: consensus on selected issues

R.G.M. Bredius, E. Jonker, C. Peters, B. Gaspar, M. van Tol on behalf of the Working Party Inborn Errors \& Pediatric Diseases

Recovery of immunity after hematopoietic stem cell transplantation (HSCT) remains poorly defined. In clinical practice, physicians are left with many uncertainties about when to stop infection prophylaxis and allow outdoor activities. It is unclear how decision making on these issues is influenced by the immune status of the HSCT recipient. A multi-round survey among members of the EBMT Inborn Errors and Pediatric Diseases Working Parties was performed using a web-based Delphi procedure, to reach a consensus on issues of infection prophylaxis strategy after pediatric HSCT that are not covered by existing guidelines.
The first survey described fictional cases covering the spectrum of HSCT conditions and complications in order to establish current policies ( $n=53$ physicians). The survey demonstrated relative agreement when initiating infection prophylaxis, while exhibiting a surprising disagreement on discontinuation of prophylaxis and permitting outdoor activities. In the second round we questioned practical rules on the most important issues, i.e. (re)vaccinations after HSCT, stopping prophylactic drugs (such as PCP and antifungal prophylaxis) and initiating activities that carry infection risk (such as outdoor play, public transportation, attending school/daycare and travel abroad), with an emphasis on immunologic recovery in addition to time after transplant as primary criteria for decision making ( $n=96$ physicians). In the third round the resulting statements were proposed, and approved or disapproved by each center ( $n=20$ SCT centers).

The consensus statements are presented (see Table).

\section{Cellular, Gene Therapies and Cytokines 2}

\section{6}

Less alloreactivity of human memory versus naive CD8

T-cells in vitro corresponds to similar data in vivo

S. Thomas, S. Klobuch, M. Sommer, M. Theobald, R.G. Meyer, W. Herr

University Medical Center of Johannes Gutenberg-University (Mainz, DE)

Reactivation of latent cytomegalovirus (CMV) infection is a frequent complication after allogeneic hematopoietic stem cell transplantation (HSCT), particularly if the donor is CMV negative and thus, no CMV-specific T cells are transferred from donor to recipient. Grafting non-reactive T cells of CMV negative donors by

\section{[0335]}

Table: Statements and consensus reached after completing round III

\begin{tabular}{|c|c|c|c|}
\hline No. & Statement & Approval* $(\%)$ & Consensus $^{\infty}$ \\
\hline 1 & $\begin{array}{l}\text { Co-trimoxazole (as PCP prophylaxis) can be stopped after HSCT when CD4+ counts are } \\
\text { stable }>300 / \mu \mathrm{L} \text {; the patient is off immunosuppressive drugs and not experiencing GVHD. }\end{array}$ & 100 & Yes \\
\hline 2 & $\begin{array}{l}\text { Antifungal prophylaxis (itraconazole or otherwise) can be stopped: at least } 2 \text { months after } \\
\text { HSCT, when ANC is sustained }>1000 / \mu \mathrm{L} \text {; the patient is off steroids; and is not } \\
\text { experiencing GVHD. }\end{array}$ & 89 & Yes \\
\hline 3 & $\begin{array}{l}\text { Outdoor play with parents (not with peers) can be allowed upon discharge from hospital } \\
\text { after HSCT when the patient has stable ANC }>1000 / \mu \mathrm{L} \text {; and is either off high-dose steroids } \\
\text { or receiving concurrent antifungal prophylaxis. }\end{array}$ & 100 & Yes \\
\hline 4 & $\begin{array}{l}\text { Other outdoor activities (restaurants, public transport, school/daycare) can be allowed } \\
\text { when: the patient is off steroids; is not experiencing active GVHD*; has stable ANC } \\
>1000 / \mu \mathrm{L} ; \text { and has stable CD4+ counts }>300 / \mu \mathrm{L} \text {. }\end{array}$ & 89 & Yes \\
\hline 5 & $\begin{array}{l}\text { Travel abroad, to most countries, can be allowed when: the patient is off steroids and not } \\
\text { experiencing active GVHD*; was successfully vaccinated against DTP/MenC/Hib/PnC }{ }^{\star *} \text {; } \\
\text { has stable ANC }>1000 / \mu \mathrm{L} \text { and stable CD } 4+\text { counts }>300 \text { cells } / \mu \mathrm{L} \text {. }\end{array}$ & 84 & Yes \\
\hline 6 & $\begin{array}{l}\text { Seasonal influenza and DTP/MenC/Hib/PnC vaccination can be started when: the patient is } \\
\text { at least } 3-6 \text { months after } \mathrm{HSCT} \text {; has no major infections at the time of vaccination; does } \\
\text { not receive IVIG; has IgM serum level } \geq 0.5 \mathrm{~g} / \mathrm{L} \text { (minor criterion); and has stable CD3+ cell } \\
\text { counts }>500 / \mu \mathrm{L} \text { (minor criterion). }\end{array}$ & 89 & Yes \\
\hline 7 & $\begin{array}{l}\text { Pneumococcal polysaccharide vaccination can be started at least }+1 \text { year after } \mathrm{HSCT} \\
\text { when: conjugate vaccination was successful }{ }^{5} \text {; the patient has no other infections at that } \\
\text { time; has endogenous IgM production of } \geq 0.5 \mathrm{~g} / \mathrm{L} \text {; and has } \mathrm{CD} 4+\text { counts }>300 / \mu \mathrm{L} \text {. }\end{array}$ & 89 & Yes \\
\hline $\begin{array}{l}= \\
\infty \\
\infty \\
=\infty \\
\$\end{array}$ & \multicolumn{3}{|l|}{$\begin{array}{l}\text { Approval is defined as the percentage of respondents evaluating the statement with a score of } \leq 3 \text { on the Likert" scale. } \\
\text { Consensus is defined as an approval rating of at least } 80 \% \text {. } \\
\text { Very mild chronic GvHD may not be considered active. } \\
\text { Alternatively, the patient may be on prophylactic IVIG and/or antibiotic prophylaxis (e.g. co-trimoxazole). } \\
\text { Successful vaccination is defined as havina protective antibody titers. }\end{array}$} \\
\hline
\end{tabular}


virus-specific T-cell receptors (TCR) may be an efficient means to transfer CMV specific T-cell function into the patient. For this, we used in vitro transcribed RNA encoding CMV-specific TCR for electroporation of human $\mathrm{T}$ cells that resulted in potent effector functions against CMV-infected targets for up to one week. Due to the transient expression of the introduced RNA, a potential study protocol would have to include the weekly administration of TCR redirected T cells. However, this approach might be hampered by the induction of serious alloreactivity through the repeated transfer of polyclonal donor T cells. To address this concern, we generated TCR transfected naive and memory T-cell subpopulations. The latter have been reported to induce less alloreactivity due to a more restricted endogenous TCR repertoire. Although both naive and memory T-cell subsets showed comparable TCR expression upon RNA transfection, memory $T$ cells mediated superior cytotoxicity against CMV-infected targets. In addition, we analyzed alloreactivity of the naive and memory T-cell subsets against HLA-mismatched EBV-transformed B-cells in IFNg ELIspot and cytotoxicity assays. As expected, alloreactivity was mainly mediated by $T$ cells of naive phenotype. To investigate the relevance of these findings for a clinical application, we used a mouse model that allows the analysis of T-cell alloreactivity directed against human hematopoiesis. For this, we reconstituted NOD/SCID/IL2Rgcnull (NSG) mice with human CD34+ stem cells and adoptively transferred them with naive and memory CD8 T-cell populations previously stimulated against cells of the stem cell donor. In line with the in vitro data, naive $T$ cells mediated a stronger alloreactivity against the donor hematopoiesis than memory T cells did. This was shown by a significant decrease of human CD45+ hematopoietic cells and $\mathrm{B}$-cells in spleen and bone marrow of the mice. Our data show that human memory CD8 T-cell populations mediate decreased alloreactivity in vitro as well as in vivo. This observation along with strong effector function upon TCR transfer makes memory CD8 $\mathrm{T}$ cells promising tools for adoptive immunotherapy.

\section{7}

Naive-derived memory stem T-cells: a novel promising platform for immune-gene therapy approaches N. Cieri (1), B. Camisa (1), F. Cocchiarella (2), M. Forcato (3), E. Provasi (1), A. Bondanza (1), C. Bordignon (4), F. Mavilio (5), A. Mondino (1), S. Bicciato (3), A. Recchia (5), C. Bonini (1) (1)San Raffaele Scientific Institute (Milan, IT); (2)Center for Regenerative Medicine (Modena, IT); (3)Center for Genome Research (Modena, IT); (4)MolMed SpA (Milan, IT); (5)Center for Regenerative Medicine (Milan, IT)

Memory stem T cells (TSCM) with the ability to self renew and the plasticity to differentiate into potent effector cells represent ideal weapons to treat cancer. Nonetheless, clinical-grade procedures to specifically target this T-cell population remain elusive. Here we report that it is possible to differentiate in vitro, vigorously expand and genetically engineer TSCM lymphocytes in clinically compliant conditions starting from naïve precursors. Requirements for the generation of this T-cell subset, best described as CD62L+ CCR7+ CD45RA+ CD45R0+ IL-7Ra+ CD95+, are CD3/CD28 engagement and culture with IL-7 and $\mathrm{IL}-15$. The gene expression profile and functional phenotype validate this cell population as a distinct memory $\mathrm{T}$ lymphocyte subset, hierarchically superior to central memory (TCM) and effector memory lymphocytes. To better define naive-derived TSCM function and potential in a clinically relevant context, we evaluated their ability to mediate xenogenic GvHD in vivo. Indeed, naïve-derived TSCM prove superior expansion and persistence than TCM manipulated with the same protocol, and are able to differentiate into effector cells, mediating a lethal xenogeneic GvHD with the same kinetics and intensity of unmanipulated lymphocytes. Furthermore, gene-modified TSCM are the only T-cell subset able to engraft and mediate GvHD upon serial transplantation, thus demonstrating that selfrenewal capacity is preserved even after ex vivo expansion and genetic manipulation. Finally, we identified and functionally validated the natural counterpart of our gene-modified TSCM population in healthy donors as a rare fraction of naive $T$ cells, recapitulating the CD62L+ CCR7+ CD45RA+ CD45R0+ IL-7R alpha + CD95+ phenotype. These findings pave the way for a rapid clinical translation of cancer adoptive immunotherapy approaches based on ex vivo generated TSCM lymphocytes.

\section{8}

T-cells but not KIR ligand incompatibilities are required for the CMV-induced anti-leukaemic effect in patients with AML after transplant

A. Elmaagacli, M. Koldehoff, M. Sonius, N. Steckel, M. Lindemann, R. Trenschel, M. Ditschkowski, T. Gromke, $H$. Ottinger, R. Ross, D. Beelen

University Hospital of Essen (Essen, DE)

We have previously showed that a CMV- replication (CMV-R) after T cell repleted HSCT is associated with a reduced risk for leukemic relapse in AML. Here, we first evaluated in a cohort of 64 pts the effect of a CMV-R on the cumulative relapse incidence (CIR) after in-vivo T cell depleted transplant using Campath $\circledast$, and secondly, the influence of a CMV-R on CIR in pts with KIR ligand incompatibilities in a $2 n d$ cohort of pts $(n=100)$ transplanted from HLA-MM SIB or URD.

In the 1st cohort $(n=64)$ pts after myeloablative (TBI based conditioning $n=56$, chemotherapy based conditioning $n=8$ ), T cell depleted transplant using Campath $\otimes$ did not benefit from a CMV-R in regard of CIR. The 5-yr CIR for pts with CMV-R $(n=23)$ after transplant was $57 \%$ vs $51 \%$ (n.s.) in pts $(n=41)$ in whom a CMV-R was not detected. Pts were transplanted in 1.CR $(n=18), 2$. CR $(n=21)$ or more progressive stages $(n=25)$ from HLA-identical SIB $(n=18)$ or URD $(n=32)$. Two pts received transplants from a SIB with one HLA -MM and 12 from URDs with one HLA-MM. CMV status of recipient (R) or donors (D) were in $25 \% \mathrm{R}-/ \mathrm{D}-$, , $8 \% \mathrm{R}-/ \mathrm{D}+; 25 \% \mathrm{D}+/ \mathrm{R}$ - and $42 \% \mathrm{R}+/ \mathrm{D}+$. The median age of pts was $46 \mathrm{yrs}$ and $38 \mathrm{yrs}$ for donors. 5-yr OS was statistically not different in both groups.

In the 2nd cohort of 100 AML-pts transplanted from HLA-MM URD $(n=96)$ or SIB $(n=4)$ donors a documented CMV-R was associated with a reduced 5 -yr CIR of $17.2 \%$ (95\% confidence limit [95\% CL]: 14-20) compared to $44 \%$ (95\% CL: 34-54) ( $p=.04)$. GVHD prophylaxis was performed with MTX, CSA with or without ATG $(n=46)$. The median age of pts was 49 yrs and 38 yrs for donors. Pts were transplanted in 1.CR $(n=40), 2$.CR $(n=32)$ or more progressive stages $(n=28)$ from a donor with one HLA-MM $(n=85)$ or 2 HLA-MM $(n=15)$. Although CIR ligand expression is reported to be influenced by a CMV-R, here the KIR ligand status had no influence on the CIR. Pts with $(n=23)$ or without KIR ligand incompatibility $(n=19)$ towards their donor benefit both from a CMV-R. In multivariate analysis CMV including competitive factors which influence CIR, CMV-R was confirmed as a independent predictor of relapse (hazard ratio [HR]: $0.12,95 \% \mathrm{CL}$ : $0.015-0.95, p<0.04)$ together with chronic GvHD (HR: 0.18, 95\% CL: 0.74-0.79, $p<0.018$ ).

The reduced risk for CIR translated into superior OS estimates at 3 yrs for pts with CMV-R (n.s. OS: $63 \%$ vs. $48 \%$ ). In conclusion, this report confirms the strong and independent effect of CMV-R on the leukemic relapse risk in AML-pts after transplant.

\section{9}

Recombinant human thrombopoietin promote platelet engraftment after unmanipulated haploidentical blood and marrow transplantation: a prospective randomised contolled trial

L. Xu, D. Liu, T. Han, F. Wang, Y. Wamg, C. Yan, Y. Chen, Y. Chen, Y. Sun, Y. Ji, Y. Zhang, J. Wang, X, H. Zhang, X. Huang People' hospital,Institute of Hematology Peking University (Beijing, CN)

Objective: Unmanipulated haploidentical blood and marrow transplantation is an important alternative transplantation 
strategy for patients without a HLA-matched or unrelated donor. Delayed platelet recovery following HSCT is a common problem. Thrombopoietin (TPO) is a naturally endogenous cytokine which can stimulate the differentiation of stem cell into the megakaryocyte, promote megakaryocyte proliferation and polyploidization, then ultimately increase the number of platelet. RHUTPO, as a memic of TPO, has been used to increase the number of platelet for many years, but it has not been evaluated in platelet engraftment of patients after haploidentical stem cell transplantation so far.

Design and Methods: In this prospective randomized controlled study, we determined the safety, tolerance and effect of rHuTPO when administered to patients after unmanipulated haploidentical blood and marrow transplantation. All the patients received the same conditioning protocol. For the study group patients, rHUTPO was administered daily hypodermically at a single dose of $15000 \mathrm{U}$, initiated the day after peripheral blood stem cell infusion and continued until platelet recovery to $>50,000 / u l$, or till the 21st day after HSCT.

Results: From April 2010 to June 2011, a total of 111 patients undergoing HSCT was randomly assigned to receive rHuTPO $(n=55)$ or not $(n=56)$. Serious adverse effects were not observed during the study period. All patients achieved an absolute netrophil count (ANC) of 500/ul in a median day of 12d (range, 10 to 20 days) vs $12.5 \mathrm{~d}$ (range, 9 to 20 days) respectively in the study group and controlled group $(\mathrm{P}=0.990)$. The accumulative rate of platelet recovery to $>20,000 /$ ul was significantly higher in the study group than in the controlled group $(P=0.0194)$, but the difference of platelet recovery to $>50,000 / u$ was not statically significant $(P=0.378)$. During the first 100 days, 18 patients in the study group and 14 patients in the controlled group developed idiopathic secondary post-transplant thrombocytopenia (ISPT) $(P=0.448)$. There was also no statically significant difference in acute graft-versus-host disease, chronic graft-versushost disease, cytomegalovirus infection, other complications following HSCT, and overall survival between the two groups. Conclusions: Our findings shows that rHuTPO can be used in the patients after unmanipulated haploidentical blood and marrow transplantation to promote platelet engraftment and it is safe and well tolerated.

\section{0}

Allelic variant MIF-173 CC associates with poor survival in acute myeloid leukaemia patients and after allogeneic stem cell transplantation

B. Martin-Antonio* (1), D. Romero* (1), M. Pratcorona (1), I. Espigado (2), D. Gallardo (3), A. Bosch (3), I. Buño (4), C. Martinez-Laperche (4), A. Jimenez-Velasco (5), R. De la Cámara (6), S. Brunet (7), J. Nieto (8), M. Suarez-Lledo (1), F. Fernandez-Avilés (1), C. Martinez (1), M. Rovira (1), J. Esteve (1), A. Urbano-Ispizua (1)

(1)Hospital Clinic (Barcelona, ES); (2)Hospital Virgen del Rocio (Seville, ES); (3)Hospital Josep Trueta (Girona, ES); (4)Hospital Gregorio Marañón (Madrid, ES); (5)Hospital Carlos Haya (Málaga, ES); (6)Hospital de La Princesa (Madrid, ES); (7)Hospital de Sant Pau (Barcelona, ES); (8)Hospital Morales Meseguer (Murcia, ES)

*Beatriz Martin-Antonio and Damiá Romero are equally contributed

Macrophage migration inhibitory factor (MIF) is a key activator of the innate immune system by initiating the inflammatory response. A constitutional variant (MIF-173CC) in the promoter region of MIF, which causes high levels of MIF, has a detrimental impact in the outcome of patients with severe sepsis and autoimmune diseases. We analyzed the clinical impact of MIF-173CC in acute myeloid leukemia (AML) patients treated with chemotherapy $(n=226)$, median age 50 years (range, 15-74); and in 675 patients submitted to HLA identical sibling allogeneic stem cell transplantation (allo-SCT), median age 44 years (range, 16-69). Cumulative incidence for acute GvHD III-IV, TRM, relapse and DFS was computed with cmprsk pak- kage and Kaplan-Meier. Functional studies for this variant after inflammatory and cytomegalovirus (CMV) stimuli were performed in 180 healthy individuals. In the AML group, MIF-173CC was associated with lower DFS (CC: $0 \%$ vs GG+GC: $26 \%$, $p=0.047$ ) and lower OS (CC: $0 \%$ vs $G G+G C: 23 \%, p=0.003$ ). This detrimental effect was confirmed in patients submitted to allo-SCT. Thus, patients with MIF-173CC had a higher frequency of death by sepsis or infection (CC: $60 \%$ vs GG+GC: $20 \%, p=0.003$ ), and higher TRM (CC: $44 \%$ vs GG+GC: $24 \%$, $p=0.037$ ). We further analyzed the clinical impact of MIF-173CC in two allo-SCT subgroups: those diagnosed with AML (alloSCT/AML) $(n=253)$ and those diagnosed with lymphoid malignancies (allo-SCT/Ly) $(n=115)$; in the allo-SCT/AML subgroup, patients with MIF-173CC had also a higher frequency of death by sepsis or infection (CC: $75 \%$ vs GG+GC: $15 \%, p=0.002$ ), higher aGVHD III-IV (CC: $58 \%$ vs GG+GC: 10\%, p=0.005), and higher TRM (CC: $43 \%$, vs GG+GC: $16 \%, p=0.048$ ). In the allo-SCT/Ly subgroup, patients with MIF-173CC had higher TRM (CC: $75 \%$ vs GG+GC: $27 \%, p=1.7 \times 10^{-9}$ ) and lower OS (CC: $0 \%$, vs GG+GC: $48 \%, p=0.013$ ). In all associations, multivariate analysis showed an independent risk for MIF-173CC. Functional studies showed higher inflammatory response and lower CD8+ cells activity after CMV stimuli for MIF-173CC. In conclusion, the detrimental impact of MIF-173CC is presented in the outcome of AML and allo-SCT patients, and an anomalous inflammatory response with lower lymphocyte response is found for this variant.

\section{1}

Decitabine-conditioned regulatory gamma delta T-cells provide enhanced protection from graft-versus-host disease via improved Foxp3 stability and ICOS up-regulation

Y. Hu, Y. Gu, K. Wu, L. Sheng, H. Huang

Zhejiang University School of Medicine (Hangzhou, CN)

Regulatory gamma delta T cells (gamma delta Tregs) is a novel kind of immune cells. In our previous study, we found decitabine could increase the induction of gamma delta Tregs with enhanced immunosuppression. We show here several associated mechanisms. Decitabine-conditioned gamma delta Tregs and gamma delta Tregs without decitabine condition (common gamma delta Tregs) were induced as described previously. CFSE-based assay was performed to test their ability to suppress phytoheagglutinin-activated human peripheral blood mononuclear cells (hPBMCs) proliferation. CFSE-labeled hPBMCs proliferation was significantly reduced when cocultured with gamma delta Tregs for 5 days. The inhibition rates were significantly different between decitabine-conditioned gamma delta Treg group and common gamma delta Treg group (Figure 1A). To clarify the underlying mechanisms we performed ELISA and flowcytomix to measure cytokine levels in the supernatant. We noted an elevated IL-10 and TGF-beta1 levels as well as decreased IFN-gamma and TNF-alfa levels in decitabineconditioned gamma delta Treg group (Figure 1A). We further confirmed elevated IL-10 level in the supernatant was due to increased IL-10 production in decitabine-conditioned gamma delta Tregs by western blot. ICOS levels have been reported to correlate with IL-10 synthesis. We found ICOS expression was up-regulated in decitabine-conditioned gamma delta Tregs (Figure 1B). We also found less than 3\% absolute number of decitabine-conditioned gamma delta Tregs and $12.6 \%$ common gamma delta Tregs were reduced on day 5, respectively, revealing elevated TGF-beta1 level was due to improved Foxp3 stability. Xeno-GVHD was induced by hPBMCs transfusion in NOD/SCID mice. Mice were co-injected with either decitabineconditioned gamma delta Tregs or common gamma delta Tregs at a ratio of 1:1. As a result, hPBMCs transfusion alone induced lethal GVHD with average survival time $25 \pm 8$ days while survival time were $43 \pm 5$ days and $58 \pm 7$ days in mice co-injected with common gamma delta Tregs and decitabine-conditioned gamma delta Tregs, respectively $(p<0.05)$. Mean IL-10 plasma 

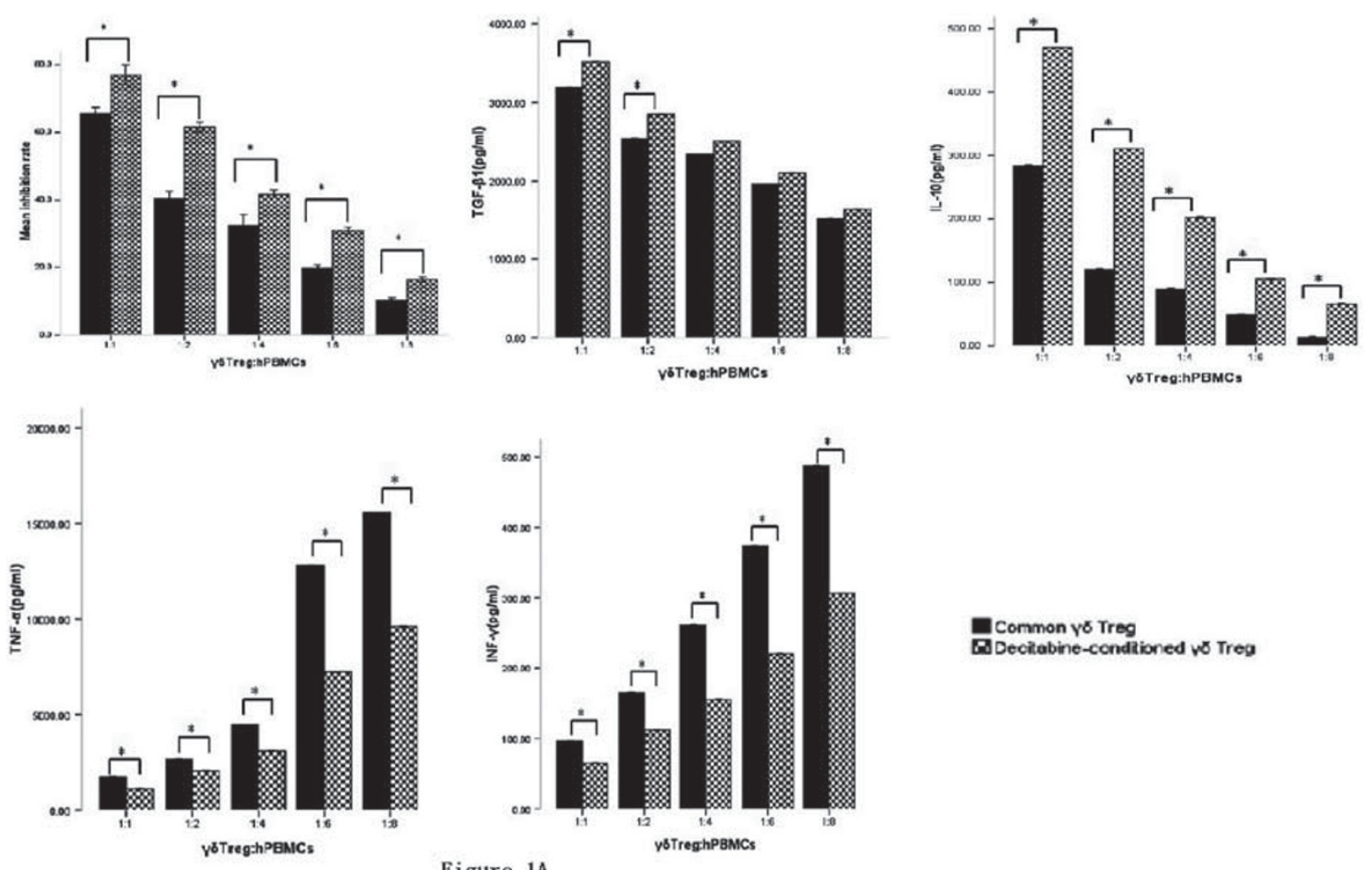

Figure 1A
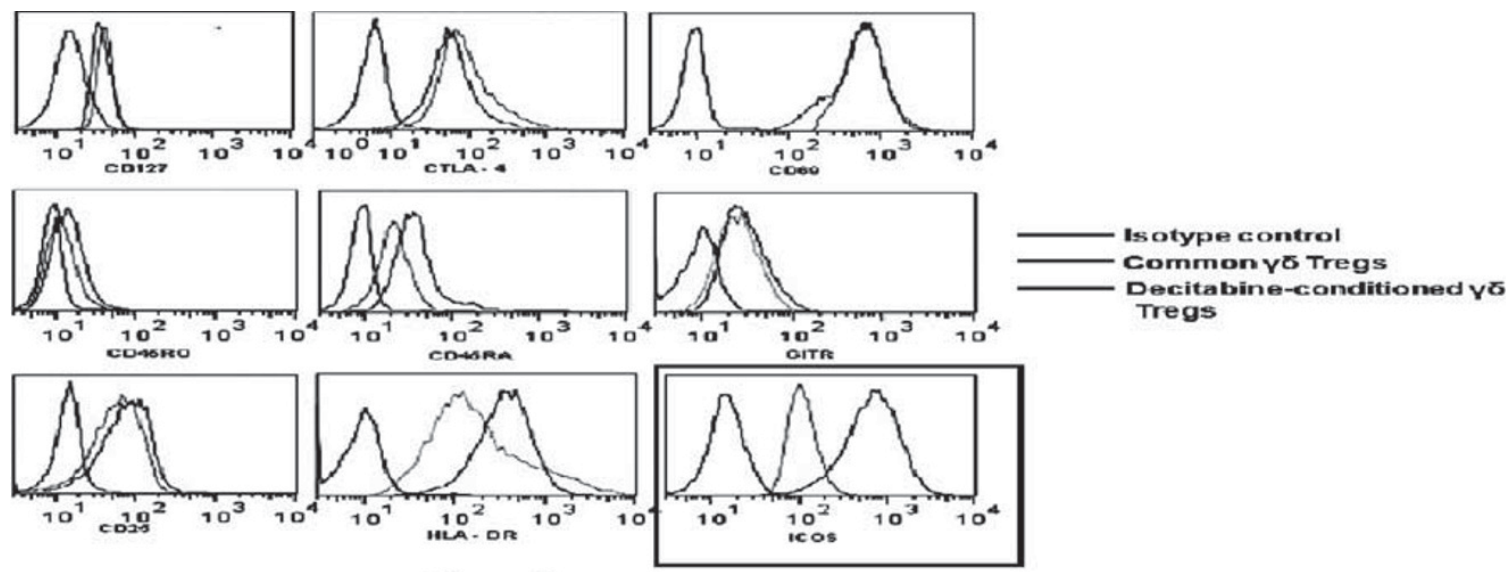

Figure 1B

levels on day 30 after hPBMCs transfusion were $178.9 \pm 11 \mathrm{pg} /$ $\mathrm{ml}$ and $25.6 \pm 7 \mathrm{pg} / \mathrm{ml}$ in mice co-injected with decitabine-conditioned gamma delta Tregs and common gamma delta Tregs respectively $(p<0.01)$. Altogether, our findings reveal that decitabine-conditioned gamma delta Tregs provide enhanced protection from GVHD via improved Foxp3 stability and ICOS up-regulation.

\section{2}

Chemotherapy breaks peripheral tolerance and allows for effective adoptive transfer of specific T-cells directed against $B$-cell restricted self-antigens

H. Dauth (1), A. Maurberger (1), C. Fahrenwaldt (2), F. Helm

(2), H. Bruns (1), F. Lehmann (3), A. Mackensen (1), C. Schmitt

(2), G. Bornkamm (3), A. Gerbitz (1)

(1)University of Erlangen (Erlangen, DE); (2)Charité Berlin (Berlin, DE); (3)Helmholtz-Center (Munich, DE)

Targeting malignant B-cell lymphomas by antigen specific T-cells is a promising strategy to improve treatment protocols. Due to the lack of lymphoma specific antigens, strategies have to rely on self-antigens as targets. This bears the problem of simultaneously eradicating non-malignant B-cells. The loss of non-malignant B-cells seems to be an acceptable side effect 
in the era of anti-CD20 antibody treatment. Malignant B-cells are difficult targets for adoptive T-cell therapy: (i) because of fast growth, they outpace T-cell responses; (ii) B-cells as antigen presenting cells are considered to render T-cells tolerant; (iii) malignant B-cells frequently possess alterations within the apoptosis machinery.

Newly developed lambda-OVA transgenic animals express a full OVA protein in B-cell specific fashion. To demonstrate tolerance towards OVA we challenged lambda-OVA animals with OVA expressing B-cell lymphoma cells. Lambda-OVA mice failed to reject OVA expressing lymphomas and died of disease progression whereas wild type littermates displayed delayed outgrowth of lymphomas and selection for antigen loss variants. Splenic B-cells from lambda-OVA transgenic mice stimulate OT-1 TCR-transgenic T-cells specific for the immunodominant peptide SIINFEKL to secrete IFN-gamma. B-cells from transgene negative control littermates or T-cells or any other hematopoietic cell from lambda-OVA mice failed to induce IFNgamma secretion by OT-1 cells. Adoptive transfer of 2.5 Mio. OT-1 cells into lambda-OVA mice did not result in expansion and B-cell counts in the peripheral blood remained normal. This suggested induction of peripheral tolerance that remained stable after immunization with OVA protein. In contrast, wild type littermates showed expansion of OT-1 cells after immunization. Treatment mice with $300 \mathrm{mg} / \mathrm{kg}$ endoxan (EDX) induced lymphopenia. Adoptive transfer of OT-1 T-cells 3 days after EDX treatment resulted in massive expansion of OT-1 cells after OVA immunization and long term depletion of $\mathrm{B}$-cells from the peripheral blood of lambda-OVA mice, whereas control littermates reconstituted their B-cell compartment within 30 days after EDX treatment.

Taken together, we show here that lambda-OVA mice are tolerant towards OVA, display a B-cell specific expression of OVA and render antigen specific T-cells anergic after adoptive transfer. This anergy can be overcome by induction of lymphopenia using EDX treatment resulting in long term depletion of B-cells in the host.

\section{Acute Leukaemia 2}

\section{3}

Long-term results of autoHSCT for adult acute

lymphoblastic leukaemia. A retrospective analysis of the Acute Leukaemia Working Party of the EBMT

S. Giebel (1), M. Labopin (2), D. Caillot (3), F. Witz (4), J. Cornelissen (5), J. Maertens (6), E. Deconinck (7), N. Ifrah (8), C. Gorin (9), M. Mohty (10)

(1)M. Sklodowska-Curie Memorial Cancer Center and Institute of Oncology, Gliwice Branch (Gliwice, PL); (2)Faculté de Médecine Saint-Antoine 27 (Paris, FR); (3)C.H.U. Dijon - Centre Georges François Leclerc (Dijon, FR); (4)C.H.R. Hôpitaux de Brabois (Vandoeuvre-Les-Nancy, FR); (5)Erasmus MCDaniel den Hoed Cancer Centre (Rotterdam, NL); (6)University Hospital Gasthuisberg (Leuven, BE); (7)Hopital Jean Minjoz (Besançon, FR); (8)C.H.R.U. - Service des Maladies du Sang (Angers, FR); (9)Hospital Saint Antoine (Paris, FR); (10)CHU Nantes (Nantes, FR)

The role of autologous HSCT in acute lymphoblastic leukemia (ALL) was subject of several prospective studies, though without clear advantage over conventional-dose chemotherapy. More recent analyses demonstrated that results of autoHSCT depend strongly on the level of minimal residual disease (MRD). Hence, in the era of routine MRD monitoring the interest in autoHSCT is re-growing. The goal of this study was to assess the long-term outcome after autoHSCT and to identify procedurerelated factors with potential prognostic significance.

440 patients, aged $35(16-65)$, with B-cell $(71 \%)$ or T-cell $(29 \%)$ ALL treated with autoHSCT in first CR between 1996-2010 were included in the analysis. 304 patients had Ph-negative and $136 \mathrm{Ph}$-positive ALL. The conditioning regimen was based on TBI in 255 (59\%) patients. Peripheral blood was used as a source of stem cells in $345(78 \%)$ cases.

With a median follow-up of 78 months, the probability of overall survival at 10 years was $42 \% \pm 3 \%$ for $\mathrm{Ph}(-)$ ALL and $17 \% \pm 4 \%$ for $\mathrm{Ph}(+) \mathrm{ALL}(\mathrm{p}<0.0001)$, while leukemia-free survival (LFS) was $35 \% \pm 3 \%$ and $12 \% \pm 3 \%$, respectively $(p<0.0001)$. The conditioning regimen did not affect the 5 year LFS rates in $\mathrm{Ph}(-) \mathrm{ALL}$ $(45 \% \pm 4 \%$ for TBI-based vs. $46 \% \pm 5 \%$ for CHT-based, $p=0.83$ ) nor $\mathrm{Ph}(+) \mathrm{ALL}(17 \% \pm 5 \%$ for TBI-based vs. $15 \% \pm 5 \%$ for $\mathrm{CHT}$ based, $p=0.58$ ). Also, no effect of the stem cells source could be demonstrated in $\mathrm{Ph}(-) \mathrm{ALL}(57 \% \pm 6 \%$ for BM vs. $45 \% \pm 4 \%$ for $\mathrm{PB}, \mathrm{p}=0.42)$ and $\mathrm{Ph}(+) \mathrm{ALL}(18 \% \pm 8 \%$ for $\mathrm{BM}$ vs. $16 \% \pm 4 \%$ for PB, $\mathrm{p}=0.45)$. Importantly, results of autoHSCT for Ph(+) ALL improved over time with $8 \% \pm 4 \%$ LFS for transplantations performed before and $23 \% \pm 5 \%$ after year $2000(p=0.03)$. In contrast, the results did not change in a setting of Ph(-) ALL.

In conclusion, a significant proportion of patients with both $\mathrm{Ph}(+)$ and $\mathrm{Ph}(-) \mathrm{ALL}$ treated with autoHSCT remain alive and disease-free after 10 years of follow-up. TBI- and non-TBIbased conditioning regimens appear equally effective. Both peripheral blood and bone marrow may be used as a source of stem cells. Improvement in the setting of $\mathrm{Ph}(+) \mathrm{ALL}$ might be associated with the introduction of tyrosine kinase inhibitors.

\section{4}

Outcomes after autologous stem cell transplantation

in AML patients using intravenous busulfan-based conditioning regimen. A survey on behalf of the

\section{ALWP-EBMT}

A. Nagler (1), M. Labopin (2), N. Gorin (3), F. Ferrara (4), A. Torrez Gomez (5), S. Lapusan (6), M. Falda (7), A. Gratwohl (8), A. Rambaldi (9), A. Botelho Sousa (10), D. Bordessoule (11), A Shimoni (1), D. Bunjes (12), P. Colombat (13), S. Cagirgan (14), P. Jacobs (15), W. Arcese (16), E. Naparstek (17), M.V. MartinReina (18), B. Bouveur (19), R. Berger (1), V. Rocha (20), M. Mohty (21)

(1)Chaim Sheba Medical Center (Tel Hashomer, IL); (2)EBMT Acute Leukemia Working Party and Registry, Hospital SaintAntoine (Paris, FR); (3)Hospital Saint Antoine (Paris, FR); (4)Cardarelli Hospital (Napels, IT); (5)Hospital Reina Sofia (Córdoba, ES); (6)Hotel Dieu (Paris, FR); (7)Azienda Ospedaliera S. Giovanni (Turin, IT); (8)University Hospital (Basel, CH); (9)Ospedali Riuniti di Bergamo (Bergamo, IT); (10)Hospital dos Capuchos (Lisbon, PT); (11)CHRU Limoges (Limoges, FR); (12)University Hospital (UIm, DE); (13)Hospital Bretonneau (Tours, FR); (14)Ege University Medical School (Izmir, TR); (15)Hospital Constantiaberg Medi-Clinic (Cape Town, ZA); (16)Rome Transplant Network (Rome, IT); (17)Tel Aviv Sourasky Medical Center (Tel Aviv, IL); (18)University Hospital Puerta del Mar (Cádiz, ES); (19)Hospital SaintAntoine (Paris, FR); (20)EBMT Acute Leukemia Working Party and Registry and Hospital Saint-Louis (Paris, FR); (21)EBMT Acute Leukemia Working Party and CHU de Nantes (Nantes, $F R)$

Busulfan (Bu) is the historical backbone of the BuCy pre- stem cell transplantation conditioning regimen. However, oral Bu has an erratic and unpredictable absorption with wide inter and also intra-patient variability. In contrast, intravenous $\mathrm{Bu}$ (iv $\mathrm{Bu}$ ) has more predictable pharmacokinetics and a favorable toxicity profile. No data is available in the literature reporting the use of iv $\mathrm{BU}$ in the autologous hematopoietic stem-cell transplantation (AutoSCT) setting. In order to do so, we performed a survey in $209 \mathrm{AML}$ pts who received iv Bu as part of the conditioning regimen. One hundred and thirteen patients were male $(46 \%)$ and the median age at transplant was 50.4 years. One hundred eight five patients $(88.5 \%)$ were transplanted in CR1, $20(9.5 \%)$ in CR2 and $4(2 \%)$ in CR3. Seventy two percent of the patients had cytogenetic intermediate risk AML while $12 \%$ and $3 \%$ had good risk and poor risk, respectively. Conditioning was 
myeloablative in all cases. Overall survival, leukemia free survival (LFS) and relapse incidence (RI) at 3 years were $57 \pm 4 \%$, $49 \pm 4 \%$ and $45 \pm 3 \%$, respectively. Only 4 patients $(1.9 \%)$ had VOD (moderate-2, severe-2) at median day 16 (range, 10-47). One of the patients died from VOD. Non relapse mortality at 3 years was low $6 \pm 1 \%$. In multivariate analysis the only prognostic factor that was found to be significant for OS, LFS, RI and NRM was age $>50$ vs $<50$ years with $p$-value of $<0.001,<0.001$, $<0.006$ and $<0.001$, respectively $(47 \pm 5 \%, 38 \pm 5 \%, 52 \pm 5 \%$, $10 \pm 3 \%$ vs $68 \pm 5 \%, 76 \pm 4 \%, 32 \pm 5 \%$ and $0 \%$, respectively). In summary, these results suggest, that similar to the allogeneic setting, VOD is a very uncommon event after AutoSCT using iv $\mathrm{Bu}$ in the conditioning regimen translating into a low NRM incidence.

\section{5}

Intravenous busulfan plus cyclophosphamide (Cyt) versus TBI plus Cy conditioning for allogeneic stem cell transplantation from matched unrelated donors. In adult patients with AML in first relapse: a survey from the

\section{ALWP of EBMT}

A. Nagler (1), M. Labopin (2), A. Shimoni (1), J. Finke (3), D. Beelen (4), L. Volin (5), J. Passweg (6), R. Schwerdtfeger (7), H. Kolb (8), A. Anagnostopoulos (9), V. Rocha (10), F. Ciceri (11), M. Mohty (12)

(1)Chaim Sheba Medical Center (Tel Hashomer, IL); (2)Hopital Saint-Antoine, and EBMT ALWP Office Universite Pierre et Matie Curie (Paris, FR); (3)University Hospital (Freiburg, DE); (4)University Hospital (Essen, DE); (5)Helsinki University Central Hospital (Helsinki, FI); (6)University Hospital Basel (Basel, CH); (7)Deutsche Klinik für Diagnostik (Wiesbaden, DE); (8)LudwigMaximilians-University (Munich, DE); (9)George Papanicolaou Hospital (Thessaloniki, GR); (10)Hopital Saint Louis APHP, EBMT ALWP and University of Paris VII (Paris, FR); (11)San Raffaele Scientific Institute (Milan, IT); (12)University of Nantes (Nantes, FR)

We compared TBI/Cy to I.V Bu/Cy conditioning prior to alloSCT from HLA matched unrelated donors in 169 adult pts with AML in Rel 1. 95 pts were given TBI/Cy and $74 \mathrm{Bu} / \mathrm{Cy}$. Median age was 38 (18-62) and 42 (19-72) years in the TBI/Cy vs. Bu/Cy groups, respectively $(P<0.005)$. FAB classification, cytogenetic risk, time from diagnosis to alloSCT, donor gender and CMV serostatus did not differ between the groups. Median year of alloSCT was 2004 vs. 2007, respectively $(P<0.001)$. ATG was used in $35 \%$ vs. $71 \%$ in the $\mathrm{TBI} / \mathrm{Cy}$ and $\mathrm{Bu} / \mathrm{Cy}$ groups, respectively $(\mathrm{P}<0.0001) .80 \%$ and $78 \%$ of the TBI/Cy and Bu/Cy groups received PBSC grafts, while $22 \%$ and $20 \%$ received BM grafts, respectively $(P=0.8)$. Median follow-up was 23 (range, 1-125) and $27(1-120)$ months in the TBI/Cy and Bu/Cy groups, respectively. Engraftment was similar, 17 (10-33) and 16 (6-31) days in the TBI/Cy and Bu/Cy groups, respectively $(P=0.23)$. Similarly, acute GVHD ( $\geq \mathrm{Gr}$ II) incidence did not differ between the 2 groups: $33 \%$ vs. $37 \%$ for the TBI/Cy vs. Bu/Cy, respectively. Death before day 100 occurred in $38 \%$ vs. $25 \%$ with TBI/Cy vs. $\mathrm{Bu} / \mathrm{Cy}$, respectively $(\mathrm{P}=0.25)$. 2y NRM was similar between the 2 groups, $28 \pm 5 \%$ vs. $19 \pm 5 \%$, respectively $(P=0.2)$. $2 y$ relapse rate was $54 \pm 5 \%$ vs. $50 \pm 6 \%$, respectively $(P=0.56)$. Induction of remission post alloSCT was higher with Bu/Cy vs TBI/Cy, $72 \%$ vs $54 \%(P=0.02)$. 2y LFS was also higher with the Bu/Cy vs TBI/Cy, groups: $23 \pm 6 \%$ vs. $18 \pm 4 \%$, respectively $(\mathrm{P}=0.045)$. Similarly, 2y OS was significantly higher with Bu/Cy vs. TBI/Cy $37 \pm 6 \%$ vs. $21 \pm 5 \%$, respectively $(P=0.013)$. The main cause of death was disease relapse: $53 \%$ and $60 \%$, with TBI/Cy vs. $\mathrm{Bu} / \mathrm{Cy}$, respectively $(\mathrm{p}=0.49)$. VOD and infection-related deaths did not differ between the groups. In multivariate analysis the interval from diagnosis to transplant ( $>v s<16$ months) was the most significant prognostic factor for Rel, LFS and OS $25 \pm 8 \%$ vs $59 \pm 4 \%(p=0.004), 48 \pm 9 \%$ vs $17 \pm 3 \%(p=0.002)$ and $41 \pm 7 \%$ vs $20 \pm 4 \%(p=0.003)$, respectively. Age, cytogenetic risk groups and use of ATG were not significant prognostic factors for survival. In all, this observational registry based study suggest that in AML pts in first Rel undergoing unrelated transplantation post transplant iv Bu/Cy vs TBI/Cy incuced higher remission rate which results in better LFS and OS. This advantage in favor of the iv Bu/Cy regimen is also possibly due to a lower overall toxicity and improved capacity for salvage therapy.

\section{6}

Impact of chronic graft-versus-host disease after reduced-intensity conditioning allogeneic stem cell transplantation for acute myeloid leukaemia: A report from the Acute Leukaemia Working Party of the European Group for Blood and Marrow Transplantation F. Baron, M. Labopin, D. Niederwieser, S. Vigouroux, J. Cornelissen, C. Malm, L. Vindelov, D. Blaise, J. Janssen, E. Petersen, G. Socie, A. Nagler, V. Rocha, M. Mohty on behalf of the Acute Leukemia Working Party

We investigated the impact of occurrence of GVHD on transplantation outcomes in a large cohort of AML pts given allogeneic PBSC after RIC conditioning. Data from 1859 AML pts in first $(n=1439)$ or second $(n=420)$ CR transplanted between 2000 and 2009 following a RIC regimen at EBMT affiliated centres were analyzed. Pts were given PBSC from HLA-identical sibling (MRD, $n=1208$ ), or from HLA-matched unrelated donors (MUD, $n=651$ ). ATG was given in $269(22 \%)$ MRD and in 267 (41\%) MUD recipients, respectively, while 151 (13\%) MRD and 165 (25\%) MUD recipients received in-vivo T cell depletion with alemtuzumab. The impact of chronic GVHD (cGVHD) on outcomes was assessed using time-dependent multivariate Cox models and in a landmark analysis at 18 months after transplant. The 3-y cumulative incidence of cGVHD was $47 \%$. Fifty-three percent of patients with cGVHD had extensive cGVHD, while the remaining $47 \%$ had limited cGVHD. In multivariate analyses, occurrence of grade II-IV aGVHD was associated with a lower risk of relapse (HR=0.8; $P=0.04)$, a higher risk of chronic $(\mathrm{HR}=2.2 ; \mathrm{P}<0.001)$ and extensive chronic $\mathrm{GVHD}(\mathrm{HR}=2.8$; $\mathrm{P}<0.001)$, a higher risk of NRM (HR=2.4 $\mathrm{P}<0.001)$, a worsened LFS (HR=1.3; $P=0.01)$, and a worsened $O S(H R=1.5 ; P<0.001)$. In multivariate time-dependent analyses, occurrence of limited cGVHD was associated with a lower risk of relapse ( $\mathrm{HR}=0.7$; $\mathrm{P}=0.05)$, comparable NRM (HR=1.4; $\mathrm{P}=0.16)$, comparable LFS $(H R=0.9 ; P=0.29)$ and better OS $(H R=0.5 ; P<0.001)$, while occurrence of extensive cGVHD was associated with a lower risk of relapse $(H R=0.6 ; P=0.01)$, higher $N R M(H R=3.2 ; P<0.001)$, a trend for worsened LFS $(H R=1.3 ; P=0.06)$ and comparable OS $(\mathrm{HR}=0.9 ; \mathrm{P}=0.34)$. In a landmark analysis in patients who were leukemia-free at 18 months after transplantation $(n=776)$, 2 -year relapse, NRM, LFS and OS were $16 \pm 2 \%, 2.5 \pm 1 \%$, $82 \pm 2 \%$, and $89 \pm 2 \%$, respectively, in patients without cGVHD before the landmark time-point, versus $9 \pm 1 \%(P=0.001), 8 \pm 1 \%$ $(P<0.001), 83 \pm 2 \%(P=0.65)$, and $86 \pm 2 \%(P=0.38)$, respectively, in patients with cGVHD before the landmark time-point. In conclusion, in this cohort of AML patients transplanted in remission, occurrence of cGVHD was associated with a lower risk of relapse that translated to better OS in patients with limited cGVHD but not in those with extensive cGVHD who experienced higher long term NRM. These results highlight the role of the GVT effect in RIC allo-SCT, but also the need for improving the prevention of severe CGVHD in pts receiving RIC allo-SCT.

\section{7}

Impact of alemtuzumab versus anti-thymocyte globulin after unrelated allogeneic stem cell transplantation with reduced-intensity conditioning as treatment for $A M L$ in CR1: a survey from the Acute Leukaemia Working Party of the EBMT

F. Baron, M. Labopin, G. Mufti, R. Arnold, C. Craddock, K. Bilger, N. Kroger, A. Nagler, M. Mohty on behalf of the acute leukemia working party

In vivo $\mathrm{T}$ cell depletion of the graft with anti-thymocyte globulin (ATG) or with alemtuzumab has been frequently used in the 
setting of RIC allo-SCT from unrelated donors. This survey compared allo-SCT outcomes between 364 AML patients in first CR given unrelated PBSC after chemotherapy-based RIC and given either ATG $(n=213)$ or alemtuzumab $(n=151)$ in the conditioning regimen. Alemtuzumab patients were more frequently given grafts from HLA-mismatched donors (30\% versus $16 \%$ having at least 1/10 HLA-mismatch with their donor, $\mathrm{P}=0.005$ ), and were conditioned more often with melphalan-based RIC $(62 \%)$, while ATG recipients were more frequently conditioned with busulfan-based RIC (84\%). Median time to neutrophil engraftment ( $>500 \mathrm{ANC}$ ) was 16 days in ATG recipients, versus 12 days in alemtuzumab recipients $(P<0.001)$. The incidence of grade II-IV acute GVHD was $28 \%$ in ATG recipients (9 patients with grade IV) and $24 \%$ (NS) in alemtuzumab recipients (2 patients with grade IV). Two-year incidences of chronic GVHD, relapse and NRM were $45 \%, 23 \%$ and $14 \%$, respectively, in ATG recipients, and $47 \%$ (NS), $25 \%$ (NS) and $25 \%$ $(\mathrm{P}=0.008)$, respectively, in alemtuzumab recipients. Two-year OS and LFS were $69 \%$ and $63 \%$, respectively, in ATG recipients, versus $55 \%(P=0.003)$ and $51 \%(P=0.02)$, respectively, in alemtuzumab recipients. Death from infection occurred in $7 \%$ of ATG recipients, versus $12 \%$ of alemtuzumab recipients. When the analysis was restricted to the 210 patients given grafts from 10/10 HLA-matched unrelated donors, the use of alemtuzumab $(n=64)$ remained significantly associated with higher NRM $(22 \%$ vs $9 \%, \mathrm{P}=0.007$ ), lower LFS ( $58 \%$ vs $69 \%, \mathrm{P}=0.07$ ), and lower OS $(62 \%$ vs $74 \%, \mathrm{P}=0.04)$. In multivariate analyses (performed in patients given grafts from 10/10 HLA-matched donors), in comparison to the use of ATG, the use of alemtuzumab was associated with higher NRM ( $\mathrm{HR}=2.5, \mathrm{P}=0.025)$, a statistically non-significant but higher relapse rate $(\mathrm{HR}=1.7, \mathrm{P}=0.18)$, and significantly worse LFS $(\mathrm{HR}=0.5, \mathrm{P}=0.013)$ and $\mathrm{OS}(\mathrm{HR}=0.4$, $\mathrm{P}=0.002$ ). In summary, this homogeneous cohort of AML patients transplanted in first CR and given PBSC grafts from unrelated donors, the use of alemtuzumab in comparison with ATG was associated with worse LFS and OS.

\section{8}

Improved survival by allogeneic haematopoietic stem cell transplantation versus autologous HSCT or chemotherapy as consolidation therapy in AML CR1 patients aged 40-60 years: the role of reduced-intensity conditioning and leukaemia risk category

J.J. Cornelissen (1), A. Gratwohl (2), K.A.G.M. van Montfort (1), T. Pabst (3), J. Maertens (4), M. van Marwijk Kooy (5), P.W. Wijermans (6), B.J. Biemond (7), D.A. Breems (8), E. Vellenga (9), L.F. Verdonck (5), M. Fey (3), H. Schouten (10), M. Jongen-Lavrencic (1), G.A. Huls (9), J.J.W.M. Janssen (11), J.H.E. Kuball (12), J.R. Passweg (2), C. Graux (13), G.J. Ossenkoppele (11), B. Löwenberg (1)

(1)Erasmus Medical Center (Rotterdam, NL); (2)University Hospital (Basel, CH); (3)University Hospital (Berne, CH); (4)University Hospital (Leuven, BE); (5)Isala Hospital (Zwolle, NL); (6)Haga Hospital (The Hague, NL); (7)Academic Medical Center (Amsterdam, NL); (8)Hospital Network Campus Stuivenberg (Antwerp, BE); (9)University Medical Center (Groningen, NL); (10)University Medical Center (Maastricht, NL); (11)VU Medical Center (Amsterdam, NL); (12)University Medical Center (Utrecht, NL); (13)UCL Mont Godinne (Yvoir, $B E)$

Patients (pts) with AML in CR1 currently qualify for alloHSCT in case of intermediate or poor-risk AML. Earlier, we showed limited benefit by alloHSCT in pts $>40 \mathrm{yrs}$, due to increased non-relapse mortality (NRM) (Blood 2007; 109:3658). Since, transplant outcome has improved and reduced intensity conditioning $(\mathrm{RIC})$ regimen were introduced in this age category by several centers. The HOVON/SAKK group set out to address the question whether alloHSCT would result in better outcome as compared to autoHSCT or chemotherapy (CT) with integrated comparison of myeloablative (MAB) versus RIC and of autoHSCT versus CT. Patients $(n=1105)$ with $A M L$ in CR1, aged 40-60 years, entered in 4 prospective HOVON/SAKK trials, were studied, including 237 pts proceeding to MAB and 144 to RIC alloHSCT. 724 pts were consolidated with either a third cycle of CT $(n=470)$ or autoHSCT $(n=254)$. More pts with poorrisk AML proceeded to alloHSCT than to autoHSCT/CT. Recipients of MAB or RIC were comparable as regards AML-risk and EBMT-risk-score, but differed with respect to use of T-celldepletion (TCD) and year of transplant. A trend towards more chronic GVHD was observed among recipients of RIC alloHSCT $(49 \%$ vs $39 \%, p=0.07)$. Patients with alloHSCT showed better OS $(56 \% \pm 3)$ than pts receiving alternative consolidation $(46 \% \pm 2), p<0.001$, irrespective of leukemia risk, and with no difference between autoHSCT and CT. Relapse Free Survival (RFS) at 5 years estimated $55 \pm 5 \%$ following RIC alloHSCT, as determined by $36 \pm 4 \%$ relapse and $9 \pm 3 \%$ NRM. RFS at 5 years following MAB alloHSCT estimated $47 \pm 3 \%$ with relapse $29 \pm 3 \%$ and NRM $24 \pm 3 \%$ at 5 years. Multivariate analysis including TCD and year of transplant showed no significant differences between RIC and MAB pts, as regards OS (HR:0.89, $p=0.55)$, RFS (HR:0.94, $p=0.75$ ), NRM (HR:0.67, $p=0.26$ ), and relapse (HR:1.05, $p=0.87$ ). In conclusion, consolidation by alloHSCT significantly improves outcome as compared to either CT or autoHSCT in AML CR1 pts aged 40-60 years, indicating that alloHSCT should be considered standard consolidation therapy in intermediate and poor-risk AML in CR1 up to the age of 60 . Since consolidation with RIC alloHSCT produces results at least as good as those following MAB alloHSCT, a prospective randomized trial of RIC vs MAB, that includes younger pts with AML in CR1 as well, is advocated.

\section{9}

Stem cell transplantation can provide durable disease control in blastic plasmacytoid dendritic cell neoplasia: a retrospective study from the European Group for Blood and Marrow Transplantation

S. Dietrich, D. Roos-Weil, A. Boumendille, E. Polge, J. Luan,

E. Carreras, A. Atienza, W. Arcese, D. Beelen, J. Cornelissen, N. Kröger, G. Milone, G. Rossi, H. Tilly, J. Vernant, V. Rocha, A. Sureda, M. Mohty, P. Dreger on behalf of the EBMT Acute Leukemia, Lymphoma and Pediatric Diseases Working Parties

Blastic plasmacytoid dendritic cell neoplasm (BPDC), formerly known as blastic NK cell lymphoma, is a rare hematopoietic malignancy preferentially involving the skin, bone marrow and lymph nodes. Most patients relapse very soon after aggressive acute leukaemia like chemotherapy but anecdotal long term remissions after consolidating myeloablative allogeneic stem cell transplantation have been reported.

Results: Within the EBMT registry 139 patients could be identified who underwent alloSCT $(n=100)$ or autoSCT $(n=39)$ for the diagnosis of BPDCN. In 74 patients histology and immunphenotyping reports could be obtained and central review confirmed the diagnosis of BPDC in 39 patients (34 alloSCT, 5 autoSCT). The 34 allografted patients were treated with a reduced intensity conditioning regimen (RIC, $n=9$ ) or myeloablative conditioning (MAC, $n=25$ ) and 19 of 34 patients were transplanted in CR1. After a median follow up time of 28 months (range: 4-77+ months), 11 patients relapsed of whom 8 died due to disease progression. 9 patients died in the absence of relapse. No relapse occurred later than 27 months after transplant. Median disease free survival (DFS) was 15 months (range: 4-77+ months) and median overall survival (OS) was 22 months (range: 8-77+ months; Figure 1a). However, long-term remissions of up to 77 months after alloSCT could be observed. Patients allografted in CR1 tended to have a superior DFS $(p=0.119)$ and OS $(p=0.057$; Figure $1 b)$. MAC was associated with a better OS $(p=0.001)$ which was attributable to the significantly higher non-relapse mortality (NRM) rate of patients after RIC ( $p=0.014)$, who had been significantly older (age RIC: 56 years, age MAC: 36 years, $p=0.0014$ ). The relapse rate was not different in patients after RIC and MAC, respectively. However, there was no survivor after RIC. 
Three of 5 patients in the autoSCT group were transplanted in CR1 of whom 1 patient relapsed after 8 months, 1 patient experienced treatment related mortality and 1 patient remained in CR for 28 months. The 2 remaining patients had more advanced disease at autoSCT and relapsed 4 and 8 months thereafter.

Conclusion: AlloSCT is effective in BPDC and might provide curative potential in this otherwise incurable disease, especially when performed in CR1. However, it remains to be shown if the potential benefit of alloSCT in BPDC is due to conditioning intensity, or if there is a relevant contribution of graft-versusleukaemia activity.
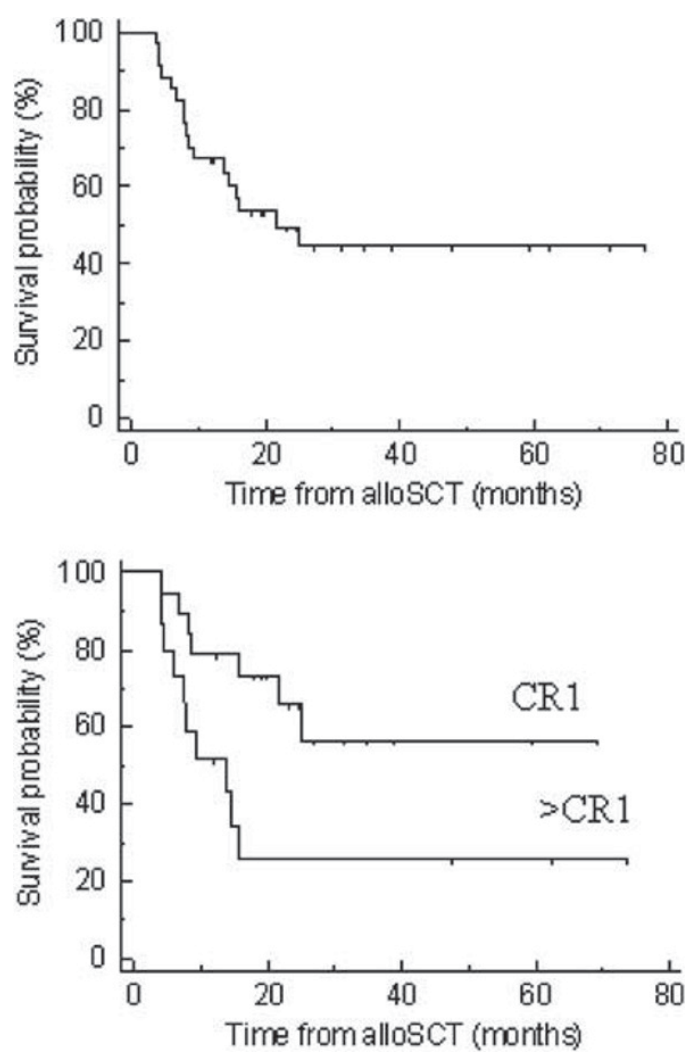

\section{Infectious Complications - Bacterial and Fungal}

\section{0}

Neutropenic enterocolitis: prospective study on usefulness of ultrasound sonography for early diagnosis and to guide medical or surgical treatment

E. Benedetti (1), F. Caracciolo (1), P. Lippolis (2), B. Bruno (3), D. Caramella (4), F. Cerri (3), F. Simonetti (1), E. Orciuolo (1), G. Buda (1), M. Pelosini (1), M. Petrini (1)

(1)Hematology Unit (Pisa, IT); (2)Surgery Division (Pisa, IT); (3)Hematology Unit (Turin, IT); (4)Radiology (Pisa, IT)

Neutropenic enterocolitis (NEC) is a life threatening complication of leukemic and solid tumors patients (pts) treated with chemotherapy (CHT) with mortality rate up to $21-48 \%$. Perforation occurs in $5 \%-10 \%$ of cases. Early diagnosis is crucial to start conservative medical management (CMM) which appears the optimal strategy for most cases. NEC should be always suspected in Neutropenic pts with abdominal pain (AP), fever $(F)$ and diarrhoea (D). Ultrasound (US) was used to evaluate bowel-wall thickening (BWT), and $>4 \mathrm{~mm}$ is considered diagnostic of NEC. Authors have proposed objective criteria for immediate surgical treatment.

Objective: Evaluate prospectively if US can detect early signs of NEC and guide a prompt treatment (CMM or surgical).

Method: in the last 4 years abdominal US was performed as only one symptom (or a combination) appeared within $12 \mathrm{~h}$ from onset: $\mathrm{F}$ and/or D and/or AP in CHT-related neutropenic patients (pts)

Results: Out of 890 pts 55 episodes were identified (6.1\%). Four pts had 2 episodes of NEC. Disease diagnosis were HD $(\mathrm{N}=10), \operatorname{ALL}(\mathrm{N}=3), \mathrm{AML}(\mathrm{N}=14), \mathrm{MM}(\mathrm{N}=7)$ and $\mathrm{NHL}(\mathrm{N}=17)$. Treatment was intensive $\mathrm{CHT}(\mathrm{N}=22)$, allogenic transplant $(\mathrm{N}=3)$, autologous transplant $(\mathrm{N}=26)$. At time of diagnosis symptoms were: $\mathrm{F}+\mathrm{AP}+\mathrm{D}$ in $72.5 \%, \mathrm{D}+\mathrm{P}$ in $15 \%, \mathrm{~F}+\mathrm{D}$ in $7.5 \%, \mathrm{~F}+\mathrm{P}$ $2.5 \%, \mathrm{P}$ in $2.5 \%$. $\mathrm{F}$ and $\mathrm{D}$ alone were never present at diagnosis of NEC. As control group we considered pts with CHT related mucositis and pts restaged with US during neutropenia in absence of symptoms. None of them had BWT. Overall 9 patients died (14.5\%). Treatment was CMM in $89 \%$ of patients and $85 \%$ survived. Six pts underwent surgery, guided by US features, during neutropenia, and $50 \%$ are alive. Median BWT was $8.3 \mathrm{~mm}$ in surviving pts and $11.1 \mathrm{~mm}$ in deceased. Median time to response from beginning of CMM was $24 \mathrm{~h}$ and the first sign of was a decrease in AP.

Conclusion: US allowed to detect early signs of NEC and to start prompt treatment in this life threatening complication, which was CMM in $89 \%$. The early recognition and intervention allowed $85 \%$ survival rate. US guided surgical intervention with $50 \%$ survival rate. Images of US and CT were superimposable. Pts who experience a $2^{\circ}$ episode of NEC have $50 \%$ chance of dying. A close clinical evaluation of the patient by Physician, Surgeon and Radiologist is mandatory. A prompt US in neutropenic patients as just one symptom appears allow to make early diagnosis of this life threatening complication and guide treatment (conservative or surgical).

\section{1}

Multiplex PCR-based assay (SeptiFast) for rapid detection of pathogens in febrile neutropenia: results in $\mathbf{2 7 3}$ consecutive patients

R. Greco (1), D. Clerici (1), N. Mancini (1), M. Clementi (2), F. Lorentino (1), L. Crucitti (1), F. Matteazzi (1), A. Forcina (1), L. Vago (1), M.T. Lupo Stanghellini (1), M. Bernardi (1), J. Peccatori (1), A. Assanelli (1), M. Carrabba (1), F. Ciceri (1), C. Corti (1)

(1)San Raffaele Scientific Institute (Milan, IT); (2)San Raffaele Scientific Institute; Vita-Salute San Raffaele University (Milan, IT)

Background: Febrile neutropenia and sepsis are frequent and life-threatening complications in patients with hematological malignancies. Blood cultures (BC) identify a pathogen in only 20 to $30 \%$ of febrile episodes, the culturing and pathogen identification process is lengthy, postponing the start of a pathogentargeted treatment. Thereby a molecular tools able to promptly recognize pathogens causing sepsis even despite ongoing antimicrobial therapy is of potential clinical relevance.

Methods: We assessed the diagnostic usefulness of the LightCycler SeptiFast test (SF), a PCR-based multiplex assay which can be performed on peripheral blood in hematological patients. In this study, blood samples from febrile oncohaematological patients were tested by traditional blood culture (BacT/Alert 3D; bioMerieux) and by a novel commercial real-time PCR assay (LightCycler SeptiFast; Roche Molecular Systems) performed concomitantly at the onset of febrile neutropenia.

Results: A total of 869 blood samples were collected from 273 consecutive patients treated for febrile neutropenia at the San Raffaele Hematology over the last three years. Out of the total 869 episodes examined, positive results were detected in 246 samples by SF $(28.3 \%)$, and in 143 by BC $(16.4 \%)$. Together, the two methods identified a total of 345 microorganisms in 312 
(36\%) episodes: Gram-positive bacterial species (74\%), Gramnegative bacterial species $(23 \%)$, and fungal species $(3 \%)$. Concordance between the two methods was $73 \%$, mainly due to samples that tested negative by culture but positive using the molecular approach (54\% of the total positive samples). The cases positive by SF alone were mostly samples from patients with initial concordant results on samples harvested before the administration of a specific antimicrobial therapy, or, importantly, sample positive for a clinically relevant agent such as Aspergillus fumigatus, which is hard to detect by the traditional approaches.

Conclusion: The LightCycler SeptiFast test gives new insights into the natural history of infection and in the development of new algorithms for the diagnosis of sepsis. Using SF combined with $\mathrm{BC}$ improves microbiological documentation in febrile neutropenia particularly in persistent fever despite antibacterial therapy, when a nonresponding bacterial infection or an invasive fungal infection is suspected; results of SF may lead to a more targeted antibiotic therapy early after the onset of fever.

\section{2}

Clinical and microbiological aspects of discontinuation of quinolone prophylaxis in patients undergoing haematopoietic stem cell transplantation: a single-centre experience

A. Verlinden, I. Vrelust, H. Jansens, A. Van de Velde, Z. Berneman, W. Schroyens, A. Gadisseur University Hospital Antwerp (Edegem, BE)

Background: Routine use of quinolone prophylaxis in neutropenic haematological patients is widespread. Although it has been reported to result in significant survival advantages, emergence of bacterial resistance has become a concern.

Objectives: The aim of this study was to evaluate the clinical and microbiological impact of discontinuing ciprofloxacin prophylaxis $(\mathrm{CP})$ in haematological patients with prolonged neutropenia. Subgroup analysis was performed on patients undergoing haematopoietic stem cell transplantation (HSCT).

Methods: Between August 2009 and July 2011 a total of 225 patients were included in this retrospective study, of which 75 underwent HSCT. 10 were excluded due to documented infection at the start of the conditioning regimen. Three sequential periods of 8 months were analysed: routine use of $\mathrm{CP}$, discontinuation of $\mathrm{CP}$ and reintroduction of CP. Patient characteristics were comparable between groups. Clinical endpoints were occurrence of neutropenic fever, bacteraemia, severe sepsis, septic shock and infection related mortality. Microbiological aspects included bacterial isolates from stool and blood cultures and their resistance pattern. Total per protocol antibiotic use for treatment of neutropenic fever and duration of hospitalisation were also analysed.

Results: The incidence of neutropenic fever, bacteraemia severe sepsis, septic shock and infection related mortality did not differ significantly between the three groups. Routine microbiological screening of stools showed a higher prevalence of pathogens in the group not receiving $\mathrm{CP}$, however these were frequently multisensitive. Quinolone resistance disappeared very rapidly after discontinuation of $\mathrm{CP}$. There was no difference in the presence of ESBL producing isolates. An expected, a rise in the occurrence of gram-negative bacteraemia was observed with discontinuation of $\mathrm{CP}$, but again with a multisensitive pattern. Antibiotic consumption and duration of hospitalisation did not differ significantly between groups.

Conclusions: Because of increasing quinolone resistance, routine ciprofloxacin prophylaxis in HSCT patients was discontinued. This did not lead to an increase in serious infectious complications nor to an increase in antibiotic consumption. Quinolone resistance disappeared very rapidly after discontinuation of ciprofloxacin prophylaxis and also reappeared very quickly after reintroduction. Analysis of this data has led us to suspend routine ciprofloxacin prophylaxis indefinitely.
0353

Palifermin after high-dose chemotherapy and autologous stem cell transplantation reduces infection rate, toxicity and resources utilisation with no increase in overall cost G. Milone (1), G. Tripepi (2), M. Poidomani (1), S. Leotta (1), R. Lombardo (1), A. Di Marco (1), S. Di Mercurio (1), S. Mercurio (1), G. Avola (1), M. Camuglia (1)

(1)Ospedale Ferrarotto (Catania, IT); (2)IBIM CNR (Reggio Calabria, IT)

We performed a matched cohort study to assess the effect of Palifermin in patients affected by haematological malignancies and who underwent high dose chemotherapy and autologous stem cell transplant (ASCT). Forty patients were treated with Palifermin and were compared to 80 matched controls. Patients treated by Palifermin and controls were matched for the two factors considered important for infection risk such as diagnosis and lenght of neutropenia after HSC infusion. Patients Treated with Palifermin had a diagnosis on Multiple Myeloma $(n=23)$, Non Hodgkin Lymphoma $(n=8)$, Acute Leukemia $(n=6)$, Chronic Lymphoid Leukemia (n. 1) or Amyloidosis (n. 2), 35\% of patients were in advanced phase of their disease while $65 \%$ in early phase. Twenty-six patients $(65 \%)$ received HD-PAM (Melphalan $200 \mathrm{mg} / \mathrm{m}^{2}$ ) and 14 patients (32.5\%) BEAM, BU-CY or HD-Thiotepa based conditioning.

Patients treated with Palifermin compared to controls had a lower rate of FUO $(p=0.02)$. Rate of „infections not related to CVC" (sum of FUO + gram negative bacteremia + pneumonia) were also significantly reduced in patients trated by Palifermin $25 \%$ versus $50 \%(P=0.01)$ and the protective effect of Palifermin remained significant also in a multiple logistic regression model $[P=0.03]$ adjusting for potential confounders. The odds ratio of severe mucositis was 30\% lower in Palifermin treated patients than in controls (odds ratio: $0.70,95 \% \mathrm{Cl}$ : $0.33-1.51$ ) but this difference was not significant $(\mathrm{P}=\mathrm{NS})$. Fewer patients treated by Palifermin had severe gastrointestinal toxicity $(12 \%$ versus $65 \%, \mathrm{P}<0.001)$, morphine utilization $(12.5 \%$ versus $38.7 \%, P<0.001)$, need for total parental nutrition (TPN) $(10 \%$ versus $68.7 \%, \quad \mathrm{P}<0.001)$ and blood products transfusions requirement $(P=0.04)$. Average economical costs related to the sum of inpatient stay, TPN, systemic antifungal treatment and blood products, were lower in Palifermin treated patients than in controls (11.985 EUROs versus 15.717, $\mathrm{P}=0.002$ ) and in Palifermin treated patients fully compensated for the cost of this drug. $75 \%$ of patients treated by Palifermin experienced skin rash that did not required interruption of administration. Overall survival in the two groups when stratified for disease status, was not different $(p=0.1)$. In conclusion, in patients undergoing High Dose Chemotherapy and PBSC transplantation, Palifermin reduces gastrointestinal toxicity, resource utilization and "infections not related to CVC".

\section{O354}

Primary prophylaxis of invasive fungal disease in allogeneic haematopoietic cell transplant recipients - a mixed treatment comparison of randomised clinical trials E. Bow (1), D. Vanness (2), C. Cordonnier (3), O. Cornely (4), D. Marks (5), A. Pagliuca (6), M. Slavin (7), C. Solano (8), L. Cragin (9), A. Shaul (10), S. Sorensen (9), R. Chambers (11), M. Kantecki (12), D. Weinstein (12), H. Schlamm (13)

(1)University of Manitoba (Winnipeg, CA); (2)University of Wisconsin and Visiting Scientist at UBC (Madison, US); (3)Hôpital Henri Mondor (Creteil, FR); (4)ZKS Cologne - BMBF 01 KN1106 (Cologne, DE); (5)University Hospitals Bristol NHS Foundation Trust (Bristol, UK); (6)King's College Hospital (London, UK); (7)Royal Melbourne Hospital (Melbourne, AU); (8)Hospital Clínico, University of Valencia (Valencia, ES); (9)UBC (Bethesda, US); (10)UBC (Manitowoc, US); (11)Pfizer (Collegeville, US); (12)Pfizer (Paris, FR); (13)Pfizer (New York, US)

Objectives: Invasive fungal infections (IFI) are a significant cause of morbidity and mortality in allogeneic hematopoietic 


\begin{tabular}{|c|c|c|c|}
\hline \multicolumn{4}{|c|}{ Estimated treatment effect on proven/probable IFI at 180 days relative to FLU } \\
\hline Comparator & $\begin{array}{l}\text { Posterior median log- } \\
\text { odds relative to FLU } \\
\text { (interquartile range)* }\end{array}$ & $\begin{array}{l}\text { Posterior } \\
\text { probability of } \\
\text { treatment being } \\
\text { better than FLU }\end{array}$ & $\begin{array}{l}\text { Posterior } \\
\text { probability of } \\
\text { treatment being } \\
\text { best of all agents }\end{array}$ \\
\hline ITR & $-0.66(-1.04,-0.28)$ & 0.84 & 0.27 \\
\hline POS & $-0.56(-1.09,-0.04)$ & 0.76 & 0.31 \\
\hline VOR & $-0.78(-1.25,-0.31)$ & 0.85 & 0.40 \\
\hline
\end{tabular}

*Estimates less than zero indicate a reduced probability of proven/probable IFI at

Day 180 relative to FLU.

cell transplant (alloHCT) recipients and these infections are difficult to diagnose and treat early. Fluconazole (FLU), itraconazole (ITR), posaconazole (POS) and voriconazole (VOR) are oral azoles that can potentially prevent IFI in this setting. The optimum choice for primary prophylaxis of IFI in alloHCT postengraftment is unknown; mixed treatment comparison (MTC) is a suitable method to evaluate this question.

Methods: Based on expert consensus, we conducted a systematic literature review of published RCTs (with $>50 \%$ of patients undergoing alloHCT) of FLU, ITR, POS and VOR as primary IFI prophylaxis. The respective cumulative proportions of patients with proven/probable IFI at 180 days were extracted from each RCT and incorporated into a Bayesian hierarchical randomeffects MTC [Lu \& Ades. Stat Med 2004; 23:3105-24]. As per expert consensus, this MTC used an unconstrained baseline to account for potential heterogeneity in study designs and patient populations. Using posterior credible intervals estimated from the MTC, we evaluated the following: 1) log-odds of developing proven/probable IFI at Day 180 for ITR, POS and VOR relative to FLU; 2) the probability that each agent was superior to FLU for this endpoint; and 3) the probability that each agent was the best of all agents.

Results: Three open-label and 2 double-blind RCTs, randomizing 2147 patients in total, were included. Based on the MTC estimates, VOR most reduced the probability of proven/probable IFI (yeasts and moulds) at day 180 relative to FLU, closely followed by ITR and POS (see Table).

Conclusion: The results suggest that mould-active azoles are more effective than FLU for preventing overall IFI incidence in alloHCT recipients post-transplant, presumably due to FLU's lack of mould activity. However, the available data did not allow us to clearly distinguish between ITR, POS and VOR in this respect. If mould coverage is desired, other considerations, such as long-term tolerability, cost and ease of use, may therefore be important when selecting the most appropriate azole for IFI prophylaxis in this setting - at least until additional data on comparative efficacy become available.

\section{5}

Can adequate prophylactic blood levels of itraconazole be achieved with capsule formulation co-administered with an acidic drink?

M. Nolan, R. Palmer, C. Steward, M. Cummins

University Hospitals Bristol Foundation Trust (Bristol, UK)

Background: Invasive fungal infection (IFI) is a leading cause of infectious death post stem cell transplantation (SCT). Antifungal prophylaxis is a key component of fungal management strategies and has been shown to improve outcome in these patients. For SCT patients with low risk of IFI, oral itraconazole is one the main antifungal prophylactic agents used.

A previous audit of practice on our unit showed intolerance to itraconazole suspension was $36 \%$. As the alternatives to itraconazole suspension are far more costly, this represented a substantial greater cost to the unit for equivalent prophylactic cover. Itraconazole capsules are not recommended in the setting of SCT due to variable bioavailability; however anecdotal reports suggested adequate blood levels could be achieved if capsules were co-administered with an acidic drink. There was no evidence base for this, and as the practice had begun to be used on the ward, this study attempts to establish whether this method of administration is a viable option for IFI prophylaxis.

Objectives: To test the null hypothesis that states 'itraconazole capsules do not achieve adequate drug serum levels and achieve inferior levels compared to itraconazole suspension'.

Method: Observational retrospective cohort study comparing two groups of SCT patients $(n=189)$ from the Bristol Bone Marrow Transplant unit. The first arm $(n=92)$ of the study received 'gold standard' itraconazole suspension; the second arm ( $n=97)$ received itraconazole capsules, co-administered with an acidic drink. Serum itraconazole levels were then objectively measured to see whether the therapeutic prophylactic level had been achieved.

Results: Therapeutic levels of itraconazole were achieved in $84.5 \%(82 / 97)$ of patients in the capsule group, compared to $61.9 \%(57 / 92)$ of patients in the suspension group. The capsule administration method was not inferior, but superior, to the suspension method. A Chi-square test of the frequencies found this result to be highly statistically significant $(p=0.0004)$. A regression analysis looked at whether sex or age could predict the level: sex was not a predictor $(p=0.64)$; age was just outside the level for statistical significance $(p=0.055)$.

Conclusion: Adequate prophylactic blood levels of itraconazole can be achieved when the capsule is co-administered with an acidic drink. This represents a viable cost-effective option offering additional flexibility for the prophylaxis of IFI in stem cell transplantation patients. 


\begin{tabular}{|c|c|c|c|c|c|}
\hline \multicolumn{5}{|c|}{ Multiple Regression of Itraconazole Levels - R-Square } \\
\hline Source & $\begin{array}{c}\text { Degrees } \\
\text { of } \\
\text { Freedom }\end{array}$ & $\begin{array}{r}\text { Type II } \\
\text { Sum of } \\
\text { Squares }\end{array}$ & $\begin{array}{c}\text { Mean } \\
\text { Square }\end{array}$ & F Value & Pr > F \\
\hline Age & 1 & 1.88 & 1.88 & 3.72 & 0.0555 \\
\hline Sex & 1 & 0.11 & 0.11 & 0.22 & 0.6391 \\
\hline Method & 1 & 3.08 & 3.08 & 6.08 & 0.0147 \\
\hline
\end{tabular}

\begin{tabular}{|c|c|c|c|c|}
\hline \multicolumn{5}{|c|}{$\begin{array}{l}\text { Blood level achieved by administration methods } \\
\text { (D.F. }=1, \text { Chi Sq }=12.37, p=0.0004 \text { ) }\end{array}$} \\
\hline \multirow{3}{*}{$\begin{array}{l}\text { Level } \\
\text { Achieved }\end{array}$} & \multirow{3}{*}{ Statistic } & \multicolumn{3}{|c|}{ Method } \\
\hline & & Itraconazole & Itraconazole & Total \\
\hline & & Capsules & Suspension & Tocan \\
\hline \multirow{2}{*}{ No } & $n$ & 15 & 35 & 50 \\
\hline & $\mathrm{Col} \%$ & 15.46 & 38.04 & \\
\hline \multirow{2}{*}{ Yes } & $n$ & 82 & 57 & 139 \\
\hline & $\mathrm{Col} \%$ & 84.54 & 61.96 & \\
\hline Total & $n$ & 97 & 92 & 189 \\
\hline
\end{tabular}

\section{6}

Gene polymorphism analysis as predictor for development of invasive aspergillosis after allogeneic stem cell transplantation

L. Gil, M. Pieronkiewicz, A. Mol, D. Poplawski, A. Schneider, K. Lewandowski, M. Komarnicki

University oF Medical Sciences (Poznan, PL)

Invasive aspergillosis (IA) continues to be a major cause of morbidity and mortality in patients undergoing alloSCT. The aim of the study was to analyze molecular risk of IA development and outcome in pts qualified for alloSCT, based on gene polymorphism studies.

Material and Methods: Two new tetra-primer ARMS-PCR's (Amplification Refractory Mutation System) were designed using Lasergene Primer Select software for TLR4 and UGTA1 SNP. IFGN and TNFR2 polymorphisms were screened with published primers but using new PCR conditions, common to all reactions. Gene polymorphisms were analyzed in a cohort of 111 pts with acute leukemias (79), chronic leukemias (10) or lymphoid malignances (22) and their sibling (53) or unrelated (58) donors. Pts were conditioned with myeloablative (50) or reduced intensity (61) regimen and grafted with 3.8 (0.9$5.2) \times 10^{6} / \mathrm{kg}$ of CD34+ cells. Standard definitions for neutropenic, bacterial, fungal and viral infections were used.

Results: Neutrophil recovery occurred in all pts at a median 21 (range; 12-38) days. Infectious complications included neutropenic fever in $102(92 \%)$, CMV infection in $45(40.5 \%)$ and documented IA in 22 (19\%) pts (proven-5 and probable-17). Acute GVHD 2-4 grade was seen in $41(37 \%)$, while chronic extensive in $9(8 \%)$ pts. The only pretransplant factor (donor/ recipient gene polymorphisms, age, sex, diagnosis, disease stage) significant for development of documented IA by logistic regression analysis was recipient TLR4 SNP ( $p=0.023$, $H R$ 4.0). Among transplant-related factors (dose and source of stem cells, intensity of conditioning, neutropenia duration, GVHD) significant for IA development was GVHD occurrence $(p=0.005, H R=5.1$ ). Over a median follow-up of 14.5 (range; 1.5-65.5) months, $89(80 \%)$ pts were alive with median survival $16.5(95 \% \mathrm{Cl}=1.5-66.6)$ months. Among 22 (20\%) deaths, 10 were related to IA. Factors significant for death from infections by univariate analysis was GVHD occurrence $(p=0.007$,
HR 3.9) documented IA $(p<0.001$, HR 7.0) and CMV infection $(p=0.034, H R 2.5)$. Factors predicting death from infection by multivariate analysis was only diagnosis of documented IA $(p=0.003, H R 23.3)$. No factors predicting the outcome of IA pts were identified.

Conclusion: High mortality rate of IA after alloSCT remains a concern. TLR4 1063A>G SNP strongly predicts IA development. IA risk analysis based on gene polymorphism may help in stratification of pts to offer an individualized treatment.

\section{Aplastic Anaemia / Autoimmune Diseases}

\section{7}

Long-term outcome after matched allogeneic

haematopoietic stem cell transplantation for Fanconi's

anaemia

R. Peffault de Latour, R. Porcher, J. Dalle, M. Aljurf, E. Korthof, J. Svahn, R. Willemze, C. Barrenetxea, V. Mialou, J. Soulier, E. Cappelli, J. Marsh, C. Peters, G. Socie, C. Dufour on behalf of the FA committee of the Severe Aplastic Anemia Working Party (SAA WP) and the Pediatric Working Party of the European Group for Blood and Marrow Transplantation (EBMT)

Allogeneic Hematopoietic Stem Cell Transplantation (HSCT) is considered the choice treatment for patients (pts) with Fanconi Anemia (FA). We evaluated the risk factors for late mortality and secondary malignancies in 1-year (yr) survivors in the largest cohort of FA pts post-HSCT ever studied, so far. Pts with FA reported to the EBMT group alive $1 \mathrm{yr}$ after a matched allogeneic HSCT were reviewed. Donor and recipient were matched if HLAA and B were identical at the generic level and HLA DRB1 at the allelic level. Cord blood as source of stem cells was excluded. Data was analyzed using proportional hazards and proportional cause-specific hazards models. Between May 1972 and January 2009, 509 FA pts alive 1 yr post-HSCT were included (273 male). Median age at HSCT was 9 yrs (range, 10 months to $44 \mathrm{yrs}$ ). $77 \%$ of pts had received stem cells from a related donor and bone marrow $(80 \%)$ was the main source of stem cells. Irradiation was used in $27 \%$ of the cohort, while fludarabine-based regimen was used in $29 \%$. T-cell depletion was used in $41 \%$. In January $2010,15 \%(n=74)$ of the pts had died. Median age at death was $19 \mathrm{yrs}$. With a median FU of 6 yrs (1 to 28 years), the probability for survival after HSCT was $49 \%$ at $20 \mathrm{yrs}(95 \% \mathrm{Cl} 38-65)$. The main causes of death were secondary malignancies in $52 \%$ of cases ( $89 \%$ of solid tumor) and treatment related mortality in $21 \%$. Cumulative incidence of death and secondary cancer are presented in Figure 1. HSCT after the age of 10 (HR 1.88 [1.17 to 3.03], $\mathrm{P}=0.009)$, clonal evolution before HSCT (HR 3.31 [1.72 to 6.39], $\mathrm{P}=0.0004)$ and previous chronic GVHD (HR 2.72 [1.65 to 4.46], $\mathrm{P}<0.0001$ ) were independently associated with decreased survival. Using occurrence of a secondary malignancy as a time-dependent covariate, the hazard of death after this event was extremely high (HR 17.3 [9.70 to 30.7], $P<0.0001$ ). Independent risk factors for secondary malignancies included HSCT after the age of 10 (HR 2.89 [1.53 to 5.45], $\mathrm{P}=0.001$ ), peripheral blood as source of stem cells (HR 3.06 [1.18 to 5.45], $\mathrm{P}=0.001$ ) and previous chronic GvHD (HR 2.89 [1.53 to 5.45], p=0.001). Longterm survival in FA pts after HSCT is still mainly affected by secondary malignancies ( $89 \%$ of solid tumors). Pts should be transplanted before the age of 10 with bone marrow as source of stem cells. Chronic GvHD still emerges as a major cause for both secondary malignancies and mortality. This study also highlights the need for very long-term FU for FA pts after HSCT. 


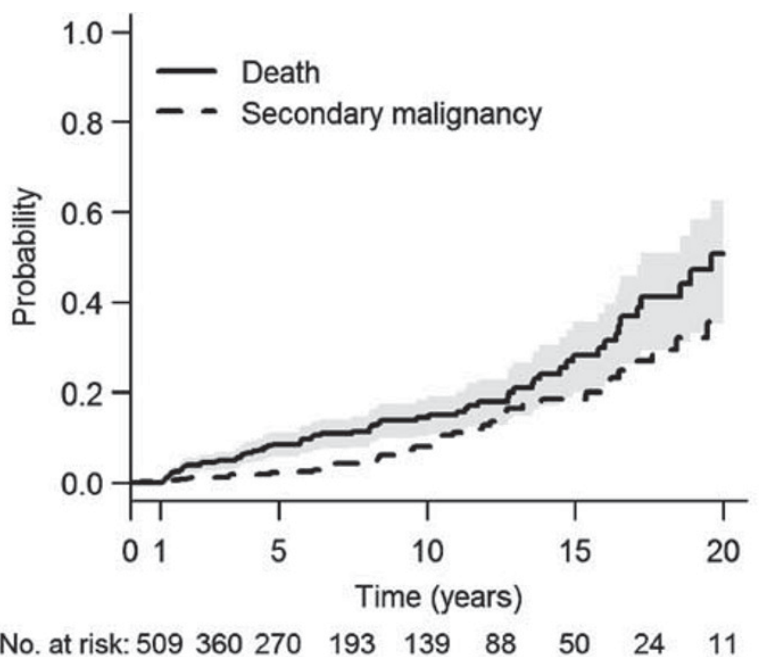

Figure 1: Cumulative incidence of death (with $95 \% \mathrm{Cl}$ ) and secondary malignancy

\section{8}

Current outcome of HLA-identical sibling versus unrelated donor transplants in severe aplastic anaemia: an EBMT analysis

A. Bacigalupo, G. Sociè, H. Schrezenmeier, A. Tichelli, A. Locasciulli, M. Fuehrer, A. Risitano, C. Dufour, J. Passweg, R. Oneto, M. Aljurf, C. Flynn, V. Mialou, R. Hamladji, J. Marsh on behalf of the WPSAA-EBMT

We have analyzed 1505 patients with acquired aplastic anemia (SAA), who received a first matched transplant between 2004 and 2009, to compare the outcome of identical sibling (SIB) $(n=980)$ vs unrelated donor (UD) $(n=525)$ transplants. Clinical characteristics of the two groups were as follows: SIB vs UD grafts had comparable age (20 vs 21 median age, $p=0.1)$ but were grafted earlier (152 vs 607 median days, $p<0.00001)$, had less frequently anti-thymocyte globulin (ATG) in the conditioning regimen $(50 \%$ vs $61 \%, \mathrm{p}<0.0001)$ and had less frequently radiation conditioning ( $25 \%$ vs $46 \%, p<0.00001)$, and more frequently received marrow as a stem cell source $(64 \%$ vs $55 \%$, $\mathrm{p}=0.002$ ).

In univariate analysis SIB transplants were superior to UD grafts $(79 \%$ vs $63 \%, p=0.007)$. However patients receiving an UD grafts were grafted significantly later, and we thus tested survival in patients grafted $\leq>>6$ months. Other variables significant in univariate analysis were patient age $(</>20)$, interval Dx-Tx(</> 180 days), stem cell source (BM vs PB) and the use of ATG.

The actuarial survival of SIB transplants $(n=584)(79 \%)$, was superior to UD grafts $(n=77)(63 \%)$ in patients with and interval Dx-TX of $\leq 180$ days, but not in patients who had an interval Dx-Tx of over 180 days (SIBs; $n=373$ vs UDs; $n=433, p=0.4$ ) (69\% for both).

We then ran a multivariate COX analysis with age, stem cell source, ATG, donor type, interval Dx-Tx. Favourable predictors for survival were, the use of BM as a stem cell source (RR 0.5, $p<0.00001)$, an interval diagnosis-transplant $(\mathrm{Dx}-\mathrm{Tx})<180$ days (RR 0.6, $p=0.006$ ), patient age $<20$ years (RR 0.6, $p<0.0001$ ), anti-thymocyte globulin (ATG) in the conditioning (RR 0.7, $p=0.01)$. Donor type (SIB vs UD) was not significant $(R R=0.8$, $\mathrm{p}=0.2$ ).

In conclusion we have shown in this study that the overall outcome of UD transplants is getting close to results achieved with SIB grafts; this is particularly true for patients with a longer interval from diagnosis (>180 days). When donor groups are corrected for age, interval Dx-Tx, use of ATG and use of stem cell source, the outcome of UDs and SIBS is comparable.

\section{9}

Long-term outcome in children with severe aplastic anaemia who received immunosuppressive therapy combined with lymphoglobuline or thymoglobuline and cyclosporine

D Jeong (1), N Chung (2), B. Cho (2), Y. Zou (3), M. Ruan (3), Y Takahashi (4), A. Ohara (5), H Kim (2), X. Zhu (3), S Kojima (4)

(1)College of Medicine, The Catholic University of Korea (Incheon, KR); (2)College of Medicine, The Catholic University of Korea (Seoul, KR); (3)Chinese Academy of Medical Science and Peking Union Medical College (Tianjin, CN); (4)Nogoya Univesity Graduate School of Medicine (Nagoya, JP); (5)Toho University Medical Center (Tokyo, JP)

Background: Thymoglobuline (TG), a rabbit anti-thymocyte globulin (ATG), has been shown to be as effective as Lymphoglobuline (LG), a horse ATG as second line immunosuppressive therapy (IST) for aplastic anemia (AA) in previous studies. However, recent prospective studies comparing horse ATG and rabbit ATG have shown that rabbitATG was inferior to horse ATG as first line treatment as measured by hematologic response rate and overall survival. These conflicting results were mainly derived from studies for adult AA. We retrospectively analyzed the clinical outcome of 453 children with AA who received LG or TG as first line IST between 1992 and 2010.

[0358]

Acquired SAA ; the effect of donor type Interval $\mathrm{DX}-\mathrm{TX}<=\mathbf{1 8 0}$ days Interval $\mathrm{Dx}-\mathrm{TX}>180$ days
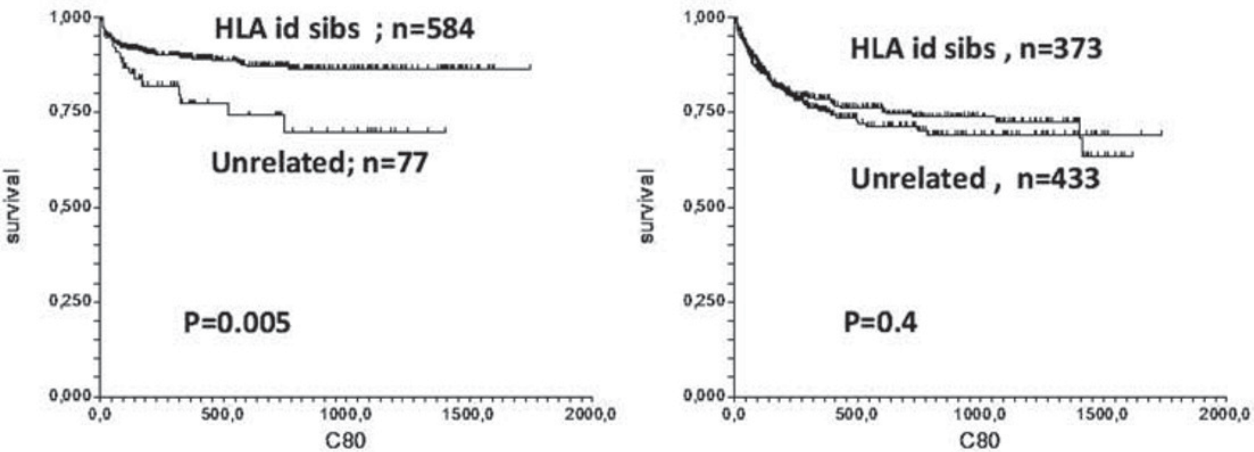
Patients and Methods: The eligibility criteria were newly diagnosed (within 6 months after diagnosis) severe AA children (younger than 18 years) who did not receive specific therapies for AA. Two hundred ninety seven children received IST consisted of LG (15 mg/kg/day for 5 days) and cyclosporine $(6 \mathrm{mg} / \mathrm{kg} /$ day for at least more than 6 months). One hundred fifty six children received IST consisted of TG $(2.3-5.0 \mathrm{mg} / \mathrm{kg} / \mathrm{day}$ for 5 days) and cyclosporine. The median age ( 8 years vs 7 years) and disease duration before initiating of treatments (16 days vs 18 days) were comparable between LG group and TG group, respectively. However, LG group suffered from more severe disease than TG group (very SAA/SAA; 166/297 vs 57/156).

Results: In the LG group, the response rate at 3 and 6 months were 136/294 (45.6\%) and 178/292 (61.3\%), respectively. In the TG group, the response rate at 3 and 6 months were $66 / 151$ $(43.7 \%)$ and $87 / 141(61.7 \%)$, respectively. The response rates were comparable between two groups $(p=0.94)$. However, 10 year overall survival rates in the TG group was significantly inferior than the LG group ( $92.1 \%$ vs $84.7 \%, p=0.01)$. Because the number of children who rescued by bone marrow transplantation (BMT) was larger in LG than the TG, we compared BMT free survival rate between two groups. When children who received BMT were censored at the time of BMT, survival rates were also significantly different between two groups $(94.4 \%$ vs $90.3 \%, \mathrm{p}=0.01$ ). Multivariate analysis for BMT free survival rates confirmed the previous result.

Conclusion: These results suggest that use of TG may be reasonable for children with SAA when horse ATG is not available.

\section{0}

Non-transplant treatment for paroxysmal nocturnal haemoglobinuria: rationale and preclinical data of the novel complement inhibitor TT30

A.M. Risitano (1), R. Notaro (2), C. Pascariello (3), M. Sica (2), L. Del Vecchio (1), M.V. Holers (4)

(1)Federico II University of Naples (Naples, IT); (2)Istituto Toscano Tumori (Florence, IT); (3)CEINGE Biotecnologie Avanzate (Naples, IT); (4)University of Colorado (Denver, US)

Objectives: The management of paroxysmal nocturnal hemoglobinuria (PNH) has drastically changed since the introduction of the first complement inhibitor eculizumab. However, the possible development of C3-mediated extravascular hemolysis (Risitano et al, Blood 2009) may limit the hematological benefit in some PNH patients. Here we provide preclinical data of a novel strategy for membrane-targeted delivery of complement inhibition.

Methods: TT30 (Alexion Pharm.) is a $65 \mathrm{kDa}$ recombinant human fusion protein consisting of the $\mathrm{iC} 3 \mathrm{~b} / \mathrm{C} 3 \mathrm{~d}$-binding region of complement receptor 2 (CR2) and of the inhibitory domain of the complement alternative pathway (CAP) regulator factor $\mathrm{H}(\mathrm{fH})$. Here we investigate the effect of TT30 on PNH erythrocytes in an in vitro model consisting of an extended acidified serum assay (EASA).

Results: We initially showed that $\mathrm{PNH}$ erythrocytes undergo lysis when incubated with normal, $\mathrm{ABO}$-matched, human serum (HNS), while sera containing eculizumab (obtained from $\mathrm{PNH}$ patients on eculizumab treatment) resulted in a partial inhibition of hemolysis. However, in these latter conditions, C3-opsonization of all PNH erythrocytes eventually occurs within $24 \mathrm{~h}$. In the EASA, TT30 demonstrated a dose-dependent complete inhibition of hemolysis; furthermore, it completely prevents C3opsonization of $\mathrm{PNH}$ erythrocytes. These data were obtained with different proportions of erythrocytes, ranging from 2 to $33 \%$ (normal-like). TT30 was twice more potent than recombinant $\mathrm{fH}$ (either of the full $\mathrm{fH}$ or of the moiety included in TT30), and an anti-CR2 monoclonal antibody (mAb) disrupting TT30 binding to C3 halved the effect of TT30. The supposed binding of TT30 to PNH erythrocytes was directly shown by flow cytometry (using both anti-fH and anti-CR2 mAbs), finally proving that TT30 works in a membrane-bound fashion.
By supplying a membrane-targeted activity of $\mathrm{fH}, \mathrm{TT} 30$ is able to disable CAP activation as soon as it starts due to spontaneous C3-tickover with subsequent C3-binding to erythrocyte surface. In PNH patients, TT30 would result in a complete protection from CAP-mediated intravascular hemolysis, as well as in prevention of possible extravascular hemolysis secondary to progressive C3-opsonization.

Conclusion: We report a novel strategy of membrane-targeted selective CAP inhibition. Our in vitro observations that TT30 inhibits both intravascular hemolysis and C3-opsonization of PNH RBCs provided the rationale for the ongoing first-inhumans phase I study in $\mathrm{PNH}$ patients.

\section{1}

Controlled trial of immunoablation and autologous haemopoetic stem cell transplantation in Crohn's disease: interim report on behalf of the ASTIC trialists. A trial from the EBMT Autoimmune Diseases Working Party

CJ. Hawkey on behalf of the Autoimmune Diseases Working Party

Background: The Autologous Stem Cell Transplantation International Crohn's Disease (ASTIC) Trial is a randomised controlled trial of immunoablation and hemopoietic stem cell transplantation (HSCT) in Crohn's disease. Recruitment of all 48 patients in the trial will complete in early 2012 and progress to date is presented.

Methods: Patients with impaired quality of life due to active Crohn's disease, despite $3+$ immunosuppressive agents all undergo mobilisation (iv cyclophosphamide $4 \mathrm{gm} / \mathrm{M}^{2}$ over 2 days followed by recombinant human granulocyte-colony stimulating factor, $10 \mu / \mathrm{kg}$ daily before randomisation to immediate (1 month) or delayed (1 year) immunoablation and HSCT. After conditioning with is iv cyclophosphamide $50 \mathrm{mg} / \mathrm{kg} / \mathrm{day}$ for 4 days, anti-thymocyte globulin $2.5 \mathrm{mg} / \mathrm{kg} / \mathrm{day}$ and methyl prednisolone $1 \mathrm{mg} / \mathrm{kg}$ on days $3-5$ an unselected graft of $3-8 \times 10^{6} / \mathrm{kg}$ CD34 positive stem cells is infused. Results are compared 1 year after mobilisation alone or after transplantation.

Results: Twelve months after stem cell transplantation the Crohn's Disease Activity Index (CDAI) fell from 324(median, interquartile range 229-411) to $161(85-257, \mathrm{n}=17)$ compared to $351(287-443)$ to $272(214-331)$ following mobilisation alone $(\mathrm{n}=11)$. Six patients had a normal CDAl after transplantation vs 1 after mobilisation. $C$ reactive protein fell from 16.6(6.7-32.0) $\mathrm{mg} / \mathrm{l}$ to $6.5(3.5-12.5) \mathrm{mg} / \mathrm{l}$ vs $14(8.0-27.0) \mathrm{mg} / \mathrm{l}$ to $9.0(2.0-23.4)$ $\mathrm{mg} / \mathrm{l}$ following mobilisation alone. The Crohn's Disease Endoscopic Index of Severity (CDEIS) (aggregate for upper and lower endoscopy) fell from $18(10-25)$ to $5(1-11)$ following transplantation vs 14(12-16) to 9(4-22) following mobilisation. Three patients achieved the goal of a normal CDAI, no drug therapy and normal upper and lower endoscopy but so did 1 patient following mobilisation alone. Serious Adverse Events were common ( $n=100$ to date) with 42 infective episodes requiring or prolonging hospitalisation, following both mobilisation and conditioning and transplantation. There were 7 episodes of viral (re)activation. Temporary flare of Crohn's disease activity or a need for surgery occurred in 8 patients.

Conclusions: Immunoablation and HSCT appears to be an effective treatment for some patients with Crohn's Disease, although full results will be required for a firm conclusion. Risks are significant, making it potentially suitable for only a limited number of patients. Data from the whole trial will be needed to judge whether mobilisation alone has any benefits. 
0362

Preliminary results of ASTIMS, a prospective randomised Phase II EBMT trial on autologous HSCT in poor prognosis Multiple Sclerosis

G.L. Mancardi (1), M.P. Sormani (1), F. Gualandi (1), A. Saiz (2), E. Carreras (2), E. Merelli (3), A. Donelli (3), A. Lugaresi (4), P. Di Bartolomeo (5), M. Rottoli (6), A. Rambaldi (7), M.P. Amato (8), L. Massacesi (8), L. Vuolo (8), D. Currò (1), L. Roccatagliata (1), M. Filippi (9), C. Russo (10), P. Iacopino (10), R. Saccardi (8)

(1)University of Genoa (Genoa, IT); (2)Hospital Clinic (Barcelona, ES); (3)University of Modena and Reggio Emilia (Modena, IT); (4)University Gabriele D'Annunzio (ChietiPescara, IT); (5)Pescara Hospital (Pescara, IT); (6)OO.RR. Bergamo (Bergamo, IT); (7)Ospedali Riuniti di Bergamo (Bergamo, IT); (8)Careggi University Hospital (Florence, IT); (9)Ospedale San Raffaele (Milan, IT); (10)Azienda Ospedaliera 'Bianchi-Melacrino-Morelli (Reggio Calabria, IT)

Autologous Stem Cell Transplantation International Multiple Sclerosis (ASTIMS) is a multicentre, prospective, randomized, single blinded phase II study that compares the activity of autologous stem cell transplantation (AHSCT): BEAM, carmustine, cytosine-arabinoside, etoposide and melphalan) versus Mitoxantrone for the treatment of patients with severe multiple sclerosis (MS) unresponsive to conventional therapy. Inclusion criteria were clinically and laboratory definite MS, with an EDSS between 3.5 and $6,5,18$ to 50 years of age, with a secondary progressive (SP) or relapsing remitting (RR) severe clinical course in the last year, defined as a deterioration of at least 1 point at EDSS or 0.5 if EDSS was > 5.5, despite conventional therapy and the presence of one or more Gd enhancing areas at MRI. The primary endpoint is the number of new T2 weighted lesions at MRI at 48 months from the randomization, while secondary endpoints are clinical progression, relapses, early and late adverse effects. The study started in 2004 and in November 2009, with 21 enrolled cases, 9 in the AHSCT and 12 in the Mitoxantrone arm, the Steering Committee decided to terminate the accrual of patients, having considered in particular, the difficulties in the enrolment of cases. Large differences in the possible side effects between the two arms, the refusal of patients to be randomized, the absence of MRI activity in the majority of SP MS patients were among the main reasons for the problems in the patients accrual. The randomized patients were followed in order to obtain the planned MRI and clinical data. Adverse events occurred in both the treatment arms, but serious adverse events were reported only in the AHSCT group under the monitoring of the DSMB. Trial safety was regularly monitored by the DSMB. An interim analysis was carried out in the first 10 randomized cases, that showed that 2 years after the completion of treatment, a mean number of 3.17 new T2 lesions in the Mitoxantrone arm vs. 0.5 in the AHSCT cases $(p<0.033)$. Four cases were lost at follow up. From December 2011,15 out of 17 evaluable patients were followed for a period longer than 48 months. Mean follow up is now 68 months (range 26-93) and final MRI and clinical evaluation will be analysed at the beginning of 2012 .

\section{3}

Immunoablation and haematopoietic stem cell transplantation in early diabetes type 1 . An update

E. Snarski (1), A. Milczarczyk (2), K. Halaburda (3), M.Paluszewska(3), T. Torosian(3), E. Urbanowska(3), M. Król(3), K. Jedynasty (2), E. Franek (2), W. Wiktor Jedrzejczak (3) (1)University of Heidelberg (Heidelberg, DE); (2)Central Hospital, Ministry of Interior Affairs and Administration (Warsaw, $P L) ;$ (3)Medical University of Warsaw (Warsaw, PL)

Background: The autologous hematopoietic stem cell transplantation in early diabetes type 1 is a new treatment modality of this autoimmune disorder. Applied early in the course of the disease has a potential to stop the destruction of beta cells and allow patients to regain exogenous insulin independency. We provide here the follow up data of 15 patients after hematopoietic stem cell transplantation in early diabetes type 1 .

Methods: The patients were mobilized with cyclophosphamide and G-CSF. The conditioning consisted of cyclophosphamide (50 $\mathrm{mg} / \mathrm{kg} /$ day on days $-5,-4,-3,-2$ prior to transplantation) and antithymocyte globulin (Thymoglobulin - of $0.5 \mathrm{mg} / \mathrm{kg} / \mathrm{day}$ given on day -5 , and $1.0 \mathrm{mg} / \mathrm{kg} /$ day given on days $-4,-3,-2$ and -1 ).

Results: The mean time of observation as of October 2011 was 26 months (range 19-40 months). No severe complications were observed during the transplantation and in the post transplantation period. Fourteen out of 15 patients became independent of exogenous insulin after the transplantation - median time without exogenous insulin for all these patients was 18,5 months (range $6-40$ months). Nine out of $15(60 \%)$ remain in remission of diabetes (exogenous insulin free) with median follow up of 26 months (range 18,5-40 months). The patients who returned to insulin have reduced requirement compared to the one prior to transplantation (mean $0,20 \mathrm{IU} / \mathrm{kg}$ versus 0,34 $\mathrm{IU} / \mathrm{kg}$ ). The average $\mathrm{HbA} 1 \mathrm{c}$ concentration was reduced in all patients after the transplantation.

Conclusion: The remission of diabetes after hematopoietic stem cell transplantation can be achieved in almost all patients with over $60 \%$ of patients remaining insulin-free 2 years after transplantation.

\section{Lymphoma}

\section{4}

Italian data on stem cell mobilisation with plerixafor in lymphoma and multiple myeloma patients: identification of prognostic factors

F. Lanza (1), F. Ciceri (2), A. Pasini (1), D. Laszlo (3), M. Montanari (4), M. Martino (5), G. Milone (6), A. Olivieri (7), P. Musto (8), V. Pavone (9), G. Specchia (10), E. Todisco (11), G. Martinelli (1), A. Bosi (12), B. Castagnari (13), A. Conconi (14), A. Cuneo (15), P. Fattori (16), A. Guerrasio (17), P. Tosi (18) (1)Hematology and BMT Unit (Cremona, IT); (2)Hematology and BMT Unit (San Raffaele Milan, IT); (3)Hematology and BMT Unit (Milan, IT); (4)Hematology and BMT Unit (Ancona, IT); (5)Hematology and BMT Unit (Reggio Calabria, IT); (6)Hematology and BMT Unit (Catania, IT); (7)Hematology and BMT Unit (Potenza, IT); (8)Hematology and BMT Unit (Rionero in Vulture (PZ), IT); (9)Hematology and BMT Unit (Tricase (Lecce), IT); (10)Hematology and BMT Unit (Bari, IT); (11)Hematology and BMT Unit (Rozzano Milan, IT); (12)Hematology and BMT Unit (Florence, IT); (13)Hematology and BMT Unit (Ravenna, IT); (14)Hematology and BMT Unit (Novara, IT); (15)Hematology and BMT Unit (Ferrara, IT); (16)Hematology and BMT Unit (Meldola (FC), IT); (17)Hematology and BMT Unit (Turin (Orbassano), IT); (18)Hematology and BMT Unit (Rimini, IT)

Plerixafor is a specific, reversible antagonist of the CXCR4 receptor, which can be used in combination with G-CSF and chemotherapy for mobilization of autologous peripheral blood hematopoietic progenitor cells (auto-PBSCT). In this study, we collected data from 211 patients with various hematological disorders (multiple myeloma - MM, n 81, non Hodgkin lymphoma, n. 105, Hodgkin's lymphoma, n 25). 144 patients were considered proven poor mobilizer, since they have had a previous mobilization failure, and 65 were classified as predicted poor mobilizers according to GITMO criteria (BMT, 2011, May 30:110). All patients received plerixafor (Mozobil-Genzyme-Sanofi) plus G-CSF with or without chemotherapy in 23 Italian centres. A total of 140 patients $(68 \%)$ collected $\geq 2 \times 10^{6} \mathrm{CD} 34+$ cells/ $\mathrm{kg}$. The collection yield was significantly higher in MM patients $(82 \%)$ than in $\mathrm{HL}(70 \%)$ and $\mathrm{NHL}(57 \%)$. The target value of $>20$ CD $34+$ cell/uL was reached in $65 \%$ of the cases after a 
median of 4.5 fold increase in CD34+ cells/uL following the use of plerixafor. The statistical analysis showed that previous treatment with radiotherapy, lenalidomide, melphalan, the number of lines of chemotherapy, age as well as leukocyte and erythrocyte counts at the time of the mobilization regimen had no significant effect on the efficiency of collection of CD34+ cells, whereas thrombocytopenia (less than $100 \times 10^{9} / \mathrm{L}$ at the time of the mobilisation attempt) and previous use of fludarabine predicted poor mobilisation with plerixafor. Eighty \% of the patients who mobilized successfully were transplanted with CD34+ cells mobilised with the combination of G-CSF and plerixafor. Short term recovery of platelet and neutrophil counts after auto-PBSCT, and safety profile post-PBSCT were found to be comparable with an historical control group transplanted with CD34+ cells which were mobilsed with growth factors and chemotherapy. These data suggest that plerixafor is safe, and may allow the rescue of lymphoma and MM patients, who need auto-PBSCT but failed CD34+ cells mobilization.

\section{O365}

Busulfan, cyclophosphamide, etoposide is not superior to carmustine, etoposide, cytarabine, mephalan conditioning prior to autologous stem cell transplantation in patients with Hodgkin's lymphoma and is associated with increased toxicity

G. Salem (1), N. Dunavin (2), L. Wei (3), P. Elder (3), S. Penza (1), K. Blum (1), P. Porcu (1), R. Baiocchi (1), J. Jones (1), J. Flynn (1), L. Andritsos (1), S.M. Devine (1), B. Christian (1), Y. Efebera (1)

(1)The Ohio State University Comprehensive Cancer Center (Columbus, US); (2)The Ohio State University Medical Center (Columbus, US); (3)The Ohio State University (Columbus, US)

Background: Busulfan $(\mathrm{Bu})$ based conditioning regimens were developed for use in Autologous Stem Cell Transplantation (ASCT) for Hodgkin Lymphoma (HL) with the goal to reduce the risk of relapse without increasing toxicity seen with conventional regimens such as Carmustine, Etoposide, Cytarabine, Melphelan (BEAM). At the Ohio State University, Bu was combined with cyclophosphamide (CY) and VP-16 (BUCYVP) and became the standard conditioning regimen here for 15 years. In 2005, we switched to BEAM regimen.

Methods: We retrospectively analyzed 179 patients (pts) with relapsed or refractory HL who underwent ASCT between 1992 and 2010. All pts received at least one chemotherapy regimen prior to ASCT. Kaplan-Meier estimates were used to analyze progression free survival (PFS), overall survival (OS). Cumulative incidence of relapse (CIR) was measured from transplant date until relapse, treating deaths as competing risks using Gray's test.

Results: One hundred eleven pts received BUCYVP (62\%) and $68(38 \%)$ pts received BEAM as a conditioning regimen prior to ASCT. Median age at transplant was 33 years with $1: 1 \mathrm{M}: \mathrm{F}$ ratio for BEAM and 2:1 for BUCYVP. Sixty two percent of BEAM pts were diagnosed at an advanced stage vs. $43 \%$ BUCYVP pts, with majority in both regiments receiving 2 lines of chemotherapy prior to transplant. Median follow-up from diagnosis and from transplant was longer for BUCYVP at 86 months (range 11-331) and 45 (0-212) months vs. 51 (18-347) and 20 (3-59) months for BEAM respectively. Pts receiving BUCYVP had higher CIR at 6 and 12 months compared to those receiving BEAM, $(p=0.04)$. Cumulative incidence of nonrelapse mortality (NRM) at 12 months was also higher for BUCYVP $(p=0.004)$. Median PFS at 1 year was $61 \%$ for BUCYVP and $85 \%$ for BEAM $(p<0.001)$ and at 3 years was $41 \%$ and $72 \%$ respectively $(p<0.001)$. Median OS from diagnosis was also longer at both 3 and 5 years for pts receiving BEAM, 92\% and $84 \%$ when compared to pts receiving BUCYVP, $81 \%$ and $67 \%$ respectively $(p=0.012)$. Adverse events were more frequent in the BUCYVP cohort. Veno-occlusive disease was significantly more common in BUCYVP-treated pts, 19\% BUCYVP pts vs.
$0 \%$ BEAM pts $(p=0.002)$. Bacterial infections were observed in $21 \%$ of BUCYVP and $13 \%$ of BEAM pts $(p=0.29)$.

Conclusion: Results of this retrospective study support the use of BEAM as the standard conditioning regimen prior to ASCT for $\mathrm{HL}$. More stringent Bu-based regimen appear to be more toxic without conveying a survival advantage.

\section{6}

Autologous stem cell transplantation for enteropathy-associated T-cell lymphoma

E. Jantunen, A. Boumendil, A. Rambaldi, M. Schapira, J.J. Luan, M. Gramatzki, S. Lenhoff, G. Öberg, P. Dreger, A. Sureda on behalf of the Lymphoma Working Party of the EBMT

Background: Enteropathy-associated T-cell lymphoma (EATL) is a rare subtype of peripheral T-cell lymphomas (PTCL) accounting for about $5 \%$ of all PTCLs in Europe. It is characterized by frequent association with celiac disease and primarily intestinal localization. The prognosis is usually poor with only $10-20 \%$ of long-term survivors. Limited data is available on feasibility and efficacy of stem cell transplantation in this lymphoma entity.

Patients and methods: The database of EBMT was used to identify patients with EATL who had received autologous and/ or allogeneic SCT in 2000-2007. All centres reporting these patients were contacted to obtain more detailed information in regard to histopathological report/pathology review and to receive information in regard to treatment before stem cell transplantation as well as the most recent follow-up.

Results: Eighty-five transplanted EATL patients were identified from the registry. Seventy-three patients had received ASCT and 12 patients allogeneic SCT. Histopathological review data and follow-up data were available in 22 ASCT patients and are included in this report. There were 14 females (64\%) and 8 males with a median age of 55 years (49-60). Half of the patients had a history of celiac disease. Ten patients had stage IV disease. The median number of treatment lines before ASCT was 1. Eleven patients (50\%) were in first complete or partial remission at the time of ASCT. BEAM was the most commonly used high-dose regimen (17 patients, $77 \%$ ). The median follow-up time was 45 months from ASCT for the living patients. Thirteen patients $(59 \%)$ have experienced a relapse or progression with a median time of only 4 months from ASCT. The median disease-free survival (DFS) was 9 months and overall survival (OS) 15 months from ASCT, respectively. OS, DFS, cumulative incidence of relapse and non-relapse mortality (NRM) at 2 years were $45 \%, 40 \%, 55 \%$ and $4 \%$.

Conclusions: ASCT is feasible in selected patients with a low NRM. About $40 \%$ transplanted patients achieve a long-term remission. This figure seems to better compared to historical series of EATL patients treated without ASCT although patient selection factors have to be taken into account. As a high number of early relapses observed after ASCT, more effective induction therapy is needed in order to improve the outcome of EATL. ASCT is a treatment option in transplant-eligible patients who respond to initial therapy.

\section{7}

High-dose therapy and autologous stem cell transplantation as consolidation after first-line treatment of HIV-associated non-Hodgkin lymphoma at high risk (aalPI 2-3). An interim report of a multicentre phase II trial A. Re, M. Michieli, B. Allione, A. Gori, S. Casari, C. Cattaneo, M. Spina, L. Verga, C. Almici, P. Ferremi, M. Mazzucato, S. Levis, U. Tirelli, G. Rossi on behalf of GICAT and FIL

Background: Outcome of aggressive HIV-associated NHL (HIV$\mathrm{NHL}$ ) with adverse prognostic features is not satisfactory with standard therapy. High dose therapy (HDT) and autologous stem cell transplantation (ASCT) has been demonstrated safe and active in HIV-NHL in salvage setting. 
[0365]
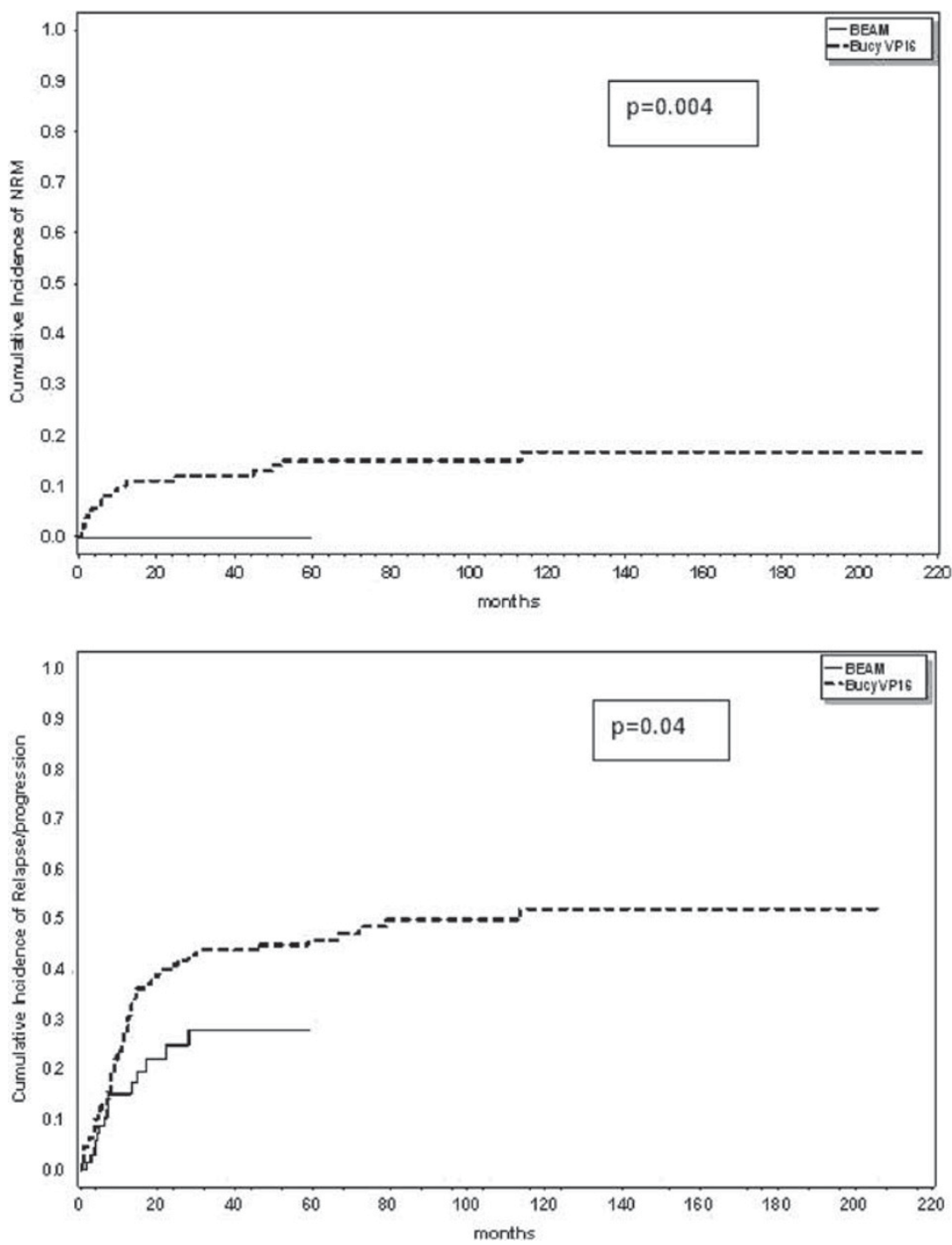

Aims: To define the role of HDT and ASCT in the upfront treatment of HIV-NHL at high risk, in terms of efficacy and toxicity. We report the interim results of a multicenter study including HDT and ASCT as consolidation after first-line treatment of HIV-NHL at high-risk.

Patients and Methods: Eligibility: untreated aggressive HIV$\mathrm{NHL}$ (including DLBCL, plasmablastic, anaplastic); aa-IPI 2-3; age<61; CD4>50/mcL; availability of effective HAART. Burkitt and CNS lymphoma are excluded. Patients (pts) receive RCHOP-21 (CHOP alone for CD20 neg lymphoma) x6 cycles and, if responsive, SC collection after CTX $4 \mathrm{gr} / \mathrm{ms}+\mathrm{G}-\mathrm{CSF}$ and BEAM. Pts receive HAART during the entire program. Overall survival (OS) at 2 ys is the primary endpoint.

Results: Since 2007 to Nov 201118 pts entered the study. 13 had DLBCL, 4 plasmablastic and 1 anaplastic. Median age 46.5 ys; PS >1 56\%; stage III/IV 22\%/78\%; LDH >n.v. 94\%; aalPI 2/3 $44 \% / 56 \%$. $72 \%$ had a prior history of HIV-positivity; $67 \%$ were on HAART at NHL diagnosis (detectable HIV 61\%); CD4 283 (58-571); HCV pos 50\%. 16/18 pts are evaluable for (R)-CHOP response ( 2 on treatment): 1 pt died with hepatic failure, 2 had prolonged cytopenia (1 with severe hepatic toxicity) that lead to withdrawal of therapy and 13 completed (R)-CHOP therapy; 9 pts had complete remission (CR), 3 partial remission (PR) and 1 disease progression (PD) (ORR 75\%, CR 56\%, PR 19\%, according to ITT). All responsive pts collected CD34+ cells; 2 had early
PD and 1 went off protocol because of cardiac EF $<50 \%$. Finally, 9 pts received ASCT according to protocol. Treatment-related toxicities ( 7 pts evaluable) included 2 grade II and 5 grade III GI and 1 grade III hepatic toxicity; 5 FUO, 1 sigmoiditis and 1 VZV infection. TRM $0 \%$. All transplanted pts are alive and relapsefree after $32 \mathrm{~ms}(1-47)$. No Ol are reported. 2-years PFS and OS of the entire series were $66 \%$ and $72 \%$ (f-up $24 \mathrm{~ms}, 2-58$ ).

Conclusions: This is the first prospective trial addressing the role of HDT and ASCT in first line treatment of HIV-LNH. The procedure was well tolerated and the clinical results highly encouraging. Interim evaluation of $\mathrm{OS}$ in this very high risk series of pts is satisfactory and accrual is ongoing.

\section{8}

Total Skin Electron Beam radiation therapy and allogeneic haematopoietic stem cell transplantation in advanced mycosis fungoides and Sézary syndrome

C. Hosing, R. Saliba, L. Singh, U. Popat, M. Qazilbash, P. Anderlini, Y. Nieto, A. Alousi, E. Frazier, B. Dabaja, R. Champlin, M. Duvic.

University of Texas, MD Anderson Cancer Center (Houston, US)

Background: Mycosis fungoides and Sézary Syndrome (SS) are incurable cutaneous T-cell lymphomas (CTCL). Role of 
allogeneic stem cell transplantation (allo-SCT) in these patients is unclear.

Patients and Methods: We conducted a Phase II trial to evaluate safety and efficacy of combining total skin electron beam (TSEB) radiation with allo-SCT in patients with advanced disease. Between 06/01 and 12/10, a total of 34 (females $=20$ \& males=14) CTCL patients underwent an allo-SCT. Median age at transplant was 53 years (20-73) and median number of prior treatments was 3 (range 1-7). Thirty-five percent (12/34) had transformed to diffuse large cell lymphoma (DLCL) and $35 \%$ had SS. Ninety one percent of patients received TSEB radiation prior to transplant and $31(91 \%)$ received the conditioning regimen fludarabine, melphalan \pm thymoglobulin. Graft-versushost-disease (GVHD) prophylaxis was with tacrolimus/ methotrexate. Median time from diagnosis to transplant was 23 months (range 4-111).

Results: Thirty patients engrafted, 2 had autologous reconstitution. Median follow up of surviving patients is 32 months (range, 2-115). The cumulative incidence of acute grade II-IV GVHD and chronic GVHD at 3 years was $41 \%(95 \% \mathrm{Cl} 27-62)$ and $44 \%(95 \% \mathrm{Cl} 29-65)$ respectively. The Kaplan-Meier estimate of OS and current progression-free survival (CPFS) at 3 years was $61 \%(95 \% \mathrm{Cl} 40-76 \%)$ and $50 \%(95 \% \mathrm{Cl} 32-68 \%)$ respectively. Sixteen patients had disease progression/relapse after transplant, 7 of whom achieved another CR after immunomodulation, second allo-SCT, and/or additional chemotherapy. These patients are alive at median follow-up of 18 months (range $2-108$ ) since progression. The cumulative incidence of progression at 3 years for patients with transformed disease was $67 \%$ vs. $34 \%$ for those who had not transformed (HR 2.5; $\mathrm{P}=0.07$ ). Patients with SS had a lower rate of progression $37 \%$ vs. $50 \%$, HR 0.5; $P=0.2$ ) although this did not reach statistical significance likely due to small numbers. At the time of this report 22 patients are alive (19 in CR and 1 with stable disease).

Conclusion: This large, single center prospective study of alloSCT in patients with advanced CTCL demonstrates long-term disease control in a subset of patients. However, relapse is common. Maintenance therapy after transplant or novel conditioning regimens may result in further improvement of these results. TSEB radiation therapy followed by allo-SCT in patients with advanced SS may impact OS.

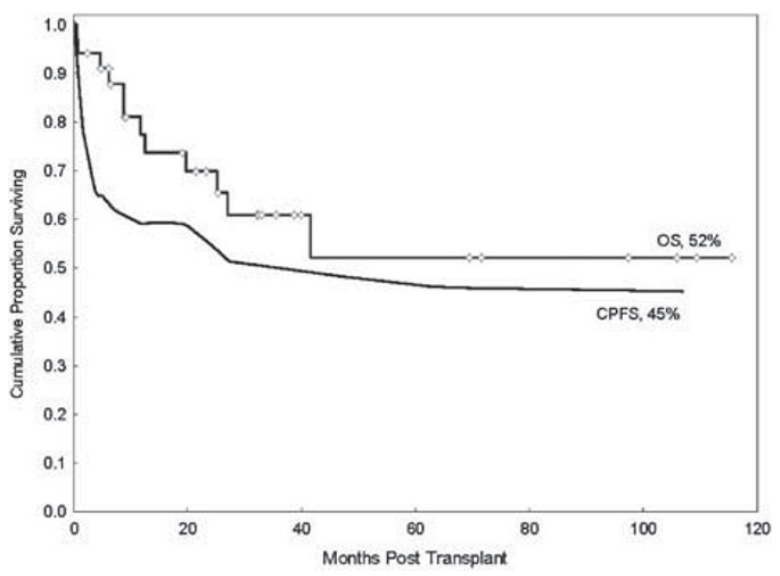

0369

Allogeneic stem cell transplantation for mantle-cell lymphoma - a report from the prospective trials \#060 and \#074 of the East German study group haematology/ oncology (OSHO)

W. Krüger (1), C. Hirt (1), N. Basara (2), H. Sayer (3), G. Behre (4), T. Fischer (5), N. Grobe (6), G. Maschmeyer (7), M. Kiehl (8), C. Busemann (1), D. Niederwieser (9), G. Dölken (1)

(1)Ernst-Moritz-Arndt-University (Greifswald, DE); (2)University Leipzig (Leipzig, DE); (3)University Jena (Jena, DE); (4)University Halle-Wittenberg (Halle/S., DE); (5)University Magdeburg (Magdeburg, DE); (6)DBK Neubrandenburg (Neubrandenburg, DE); (7)Klinikum EvB (Potsdam, DE); (8)Klinikum Frankfurt/Oder (Frankfurt/Oder, DE); (9)University Leipzig (Greifswald, DE)

Introduction: Mantle cell lymphoma (MCL) has a poor prognosis under conventional therapy. AlloSCT is here a promising approach.

Methods: Two prospective trials were conducted to investigate the efficacy of chemotherapy-based conditioning followed by alloSCT for treatment of MCL: Trial \#074 was open for patients with de-novo MCL and \#060 for patients requiring salvage therapy. At least a pR was mandatory for proceeding to alloSCT. Conditioning consisted of treosulfan $\left(12 \mathrm{~g} / \mathrm{m}^{2}\right)$ and fludarabin $(150 \mathrm{mg} /$ $\mathrm{m}^{2}$ ). Busulphan plus cyclophosphamide was optional for younger patients. ATG was given prior to mismatched or mud SCT.

Results: 39 mainly male $(n=31)$ patients with a median age of 59y (33-69) have been recruited into both trials (\#060: $n=15$, \#074: $n=24)$. In de-novo patients the median MIPI was $5(2-9)$. Salvage patients were pre-treated with 8 (6-13) cycles chemotherapy. (Re)-Induction prior to TX consisted mainly of R-CHOP or R-DHAP. 33 patients proceeded to alloTX from mrd or mud. 2 patients died from progressive disease prior to TX, 1 patient had no donor, in one case the diagnosis was revised and 2 patients were withdrawn. 26 patients $(79 \%)$ were conditioned with Treo/Flu and $7(21 \%)$ with Bu/Cy. $76 \%(n=25)$ of patients received a graft from an unrelated donor. Toxicity was moderate and incidence of acute GvHD was $51 \%$. 26 patients are well with a median $\mathrm{KI}$ of $100 \%$ (range $50 \%-100 \%$ ) and alive after SCT with a median follow-up of 18 months $(0,3-114)$ in CCR without differences between both trials. 6 patients have died from infections and cerebral bleeding $(n=1)$ and one from infection related to an acute GvHD IV ${ }^{\circ}$ due to DLI for relapse (blastic variant) in CR of MCL. Molecular analyses showed a 2-4log reduction of circulating lymphoma cells after chemotherapy alone. Blood became negative by qPCR after allo-SCT in all 5 patients analysed so far. An intermediate increase of circulating lymphoma cells in 3 patients was successfully treated by rituximab, withdrawal of Cy-A and DLI.

Conclusion: Allo-SCT is a standard salvage therapy for suitable patients and an option for patients with de-novo MCL. Longterm remissions can be reached and negativity of mrd analyses by qPCR strongly supports curative potential of allo-SCT. Close monitoring of minimal disease allows steering of immunotherapy 'on demand' and helps to avoid an overtreatment with possible associated adverse effects. GvL-effects have curative potential even in the case of relapsed blastic variant of $\mathrm{MCL}$.

\section{0}

Allogeneic stem cell transplantation as second-line therapy offers curative potential in advanced T-cell lymphomas

A. Czajczynska (1), A. Günther (1), A. Humpe (1), R. Repp (1), A. Schrauder (1), M. Nickelsen (2), T. Raff (1), M. Kneba (1), M. Gramatzki (1)

(1)University of Kiel (Kiel, DE); (2)Asklepios Hospital St Georg (Hamburg, DE)

Patients with T-cell lymphomas have a dismal prognosis when a disease relapse occurs after front line treatment. Here, the outcome of 24 patients with T- and NK-cell lymphomas treated 


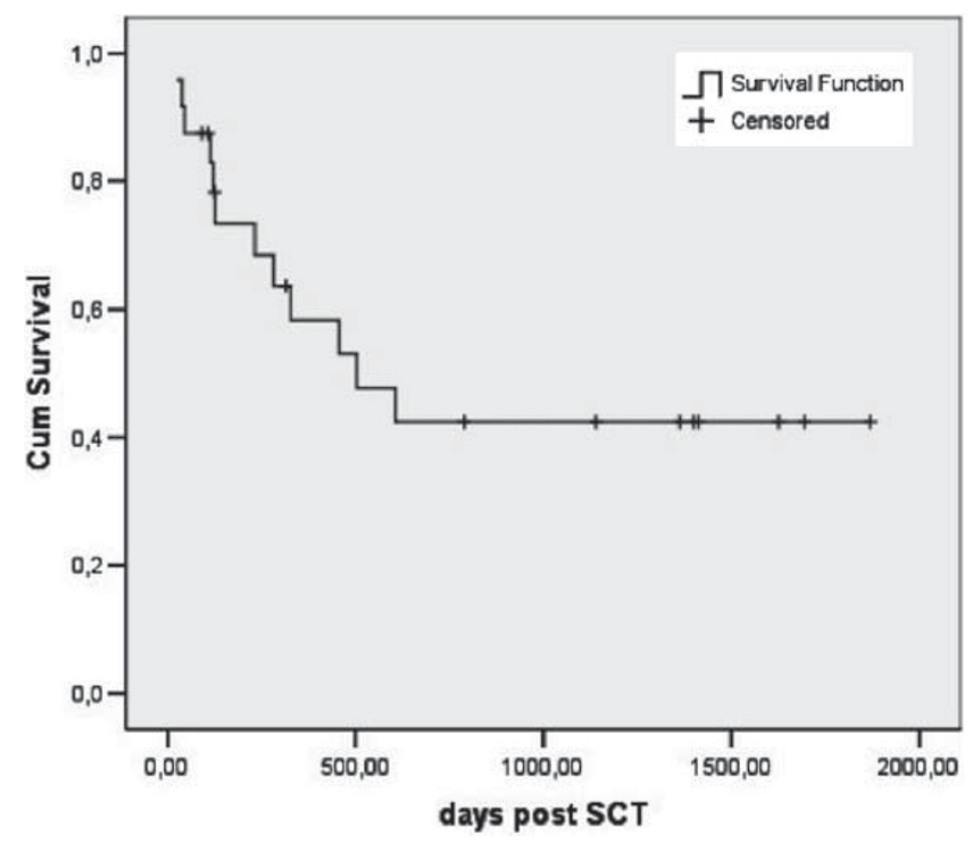

consecutively at our center in a rather uniform approach with allogeneic stem cell transplantation is presented.

Results: Patients in the age range of 11 to 65 years were included after a median of 2 prior lines of therapy with these T-NHL subtypes: ALCL-ALC+, ALCL-ALK-, EATL, extranodal NK/ T-cell nasal type, T-PLL, AITL, T-LBL and PTCL-NOS. With few exceptions, the CLAEG regimen, consisting of cladribine, cytosine arabinoside, etoposide, and mandatory G-CSF was administered for remission induction or early consolidation. As soon as possible after recovery the patients were conditioned for allogeneic transplantation with BEAM and alemtuzumab (Faulkner et al., Blood 103:428, 2004). The protocol called for a fixed dose of $50 \mathrm{mg}$ of the CD52-antibody alemtuzumab in order to provide GvHD prophylaxis and to facilitate tumor control as well. Most patients $(n=19)$ received transplants from unrelated donors, including 5 with one or more mismatches. Toxicity of the transplant procedure was moderate, although infections were frequent and the leading cause of death in two patients. After rapid engraftment only 6 patients showed equal or greater grade II acute GvHD, however one of them died of grade IV disease. Six cases of chronic GvHD occurred. In 5 patients donor lymphocyte infusions were applied. While 12 patients had never achieved a CR before transplantation, 20 of 22 evaluable patients reached a CR after transplantation. With a median follow up of 1252 days (range: day 90 to 1871), 12 patients are in continuous complete remission. For overall survival see the enclosed figure. In this group of advanced relapsed or refractory T-NHL allogeneic stem cell transplantation with BEAM-alemtuzumab conditioning is able to induce long-term remissions in a considerable proportion of patients, when performed rapidly after intense remission induction.

\section{Joint Session EBMT / WMDA}

\section{5}

Impact of earth quake/nuclear accident on stem cell transplants in Japan

Y. Kodera, S. Chiba, S. Kato, S. Taniguchi for JSHCT, JMDP and JCBBN

On JSHCT: The head quarter located in Nagoya was intact. Attacked region was the eastern region of the main island of
Japan where 17 hematopoietic stem cell transplant teams located. The earthquake minimally influenced autologous and related HSCT. The influence to unrelated HSCT would be described later. After June, the HSCT activities of this region returned to usual level. JSHCT made a quick search for the capability to accept recipients and donors from the attacked region. Finally 107 teams through the country expressed the will to accept them. JSHCT organized Nuclear Accident Committee and announced two statements including the support for autologous stem cell storage for potential victims of acute radiation injury. JSHCT sent representatives to Paris, London, Chicago and Minneapolis to report our situation. Monthly report of JSHCT showed no significant decrease of related HSCT in this period comparing to the last year.

On JMDP: The head quarter located in Tokyo was partially damaged but the function was maintained. On March 11-14, 43 recipients including 1 recipient in the attacked region were under the preconditioning but the recipient successfully received marrow from Tokyo area. Forty-two of 43 corresponding donors were confirmed to be safe but 2 including the one who could not be confirmed its safety out of 43 were switched to CBSCT. Several recipients of 22 under the final preparation moved to other areas to receive unrelated HSCT. New coordination in the region was postponed until June. JMDP set the standards of urgent coordination for the potential victims of acute radiation injury. Monthly report from JMDP showed slight decrease of unrelated HSCT from volunteer donors in March but it recovered in April.

On JCBBN: The head quarter located in Tokyo was intact. One of 11 local banks located in the eastern region (Miyagi CBS Bank) and its building got damage without the damage of nitrogen tanks. Allocation of CBS from that bank was postponed until June mostly because of the transportation problems. The other banks were basically intact and monthly report of JCBBN showed certain increase of CBSCT in March.

Summary: Although the mega-earthquake/tsunami was almost catastrophic in the eastern region of the main island, its influence to stem cell transplant activity was not critical at the country level. The nuclear accident brought us the opportunity to re-consider the role of HSCT to acute radiation injury. 